\title{
SEMIPARAMETRIC PARTIALLY LINEAR MARGINAL MODELS FOR BINARY AND COUNT LONGITUDINAL DATA WITH DROPOUTS
}

by

SEEMA ZUBAIR

A thesis submitted to the Faculty of Graduate and Postdoctoral Affairs in partial fulfillment of the requirements for the degree of

\section{Doctor of PhILOSOPHY}

IN

Probability and Statistics

Ottawa-Carleton institute for Mathematics and Statistics

Carleton University

OtTaWa, Ontario

(C) Copyright

SEEma Zubair, 2020 


\section{Abstract}

In this thesis, we investigate semiparametric partially linear marginal models for binary and count longitudinal data with dropouts. Specifically, we focus on the joint estimation of the marginal mean, association and dispersion parameters through generalized second-order estimating equations where the marginal mean response model is partially linear.

We discuss a series of weighted generalized estimating equations (GEEs) to fit regression models to binary and count longitudinal responses when dropouts occur. The proposed method offers efficient estimators of the model parameters under a specified missing data mechanism. Simulations are conducted to examine the robustness characteristics of the method suggested under both accurately defined and inaccurately stated correlation frameworks. The approach is also demonstrated using some real missing longitudinal data on patterns of smoking, where the goal is to study the development of coronary arteries in young adults.

A semiparametric approach for analyzing binary and longitudinal count data is also developed. We used the second-order GEE approach to examine longitudinal responses in partially linear models. Additionally, the smoothing technique is suggested for estimating the nonparametric part of the model based on a spline approximation. In simulations, the analytical properties of the proposed method are evaluated. The proposed estimator effectively takes into consideration the association within the subject/cluster and is easy to implement. Our simulation study shows that when the underlying model is partly linear, the proposed method offers unbiased and efficient estimators. 
Next, we propose a weighted regression spline second-order GEE approach for simultaneous estimation of the nonlinear function, regression, association and dispersion parameters in partially linear models with dropouts. As an application of the proposed semiparametric weighted GEE, we analyzed some longitudinal count data obtained from a health survey, referred to as the Health and Retirement study (HRS)(HRS, 2019), where the mean response function shows a nonlinear trend in terms of associated covariates. The results from the data analysis appear to be very encouraging. From this application it is evident that our proposed methods can be used to improve the efficiency of the estimates obtained from an ordinary GEE model for longitudinal binary and count data with dropouts. 


\section{Acknowledgments}

First and foremost, praises and thanks to God, the Almighty, for His blessings throughout my academic and research work. I would like to thank my supervisor Dr. Sanjoy K. Sinha for all his guidance, encouragement and readiness to lend a hand in writing my thesis. His dynamism, vision, sincerity and research knowledge have inspired me. He has taught me the methodology to carry out the research and to present the research works in the clearest and most professional ways. It was a great privilege and honour to work and study under his guidance.

I am thankful to the Dean, director, faculty, and staff members of the Department of Mathematics and Statistics, for their help during my study and research through academic and financial support. Furthermore, I am also thankful to my dear friends and colleagues, whose support is highly appreciated in the compilation of this dissertation.

I would like to express my eternal appreciation towards my parents, siblings and in-laws who have always been there for me no matter where I am, for unconditional support and patience. Nobody has been more important to me in the pursuit of this thesis than my family members. Most importantly, I want to thank my loving and supportive husband, Muhammad Zubair, for being understanding, supportive and for never-ending motivation that I have been getting all this while. And, last but not least, my three wonderful children, Abeeha, Eeshal and Yahya, who have been an unending source of inspiration. 
I dedicate this thesis to my late parents

Mr \& Mrs Mohammad Iqbal

for their support and prayers from the day one of my school. May their souls find highest place in Paradise. 


\section{Contents}

Abstract

Acknowledgments

Dedication $\quad$ iv

Table of Contents $\mathbf{x}$

List of Abbreviations $\quad$ xi

List of Tables xiv

List of Figures $\quad$ XV

1 Introduction 1

1.1 Longitudinal Studies . . . . . . . . . . . . . . . . . . . . . . 1

1.2 Dropouts in Longitudinal Data . . . . . . . . . . . . . . 2

1.3 Marginal Models for Longitudinal Data . . . . . . . . . . . . . . . . . 3

1.4 Semiparametric Models for Longitudinal Data . . . . . . . . . . . . . 4

1.5 Statement of the Problems . . . . . . . . . . . . . . . 5

1.6 Organization of Thesis . . . . . . . . . . . . . 6

2 Overview of Generalized Estimating Equations for Longitudinal

$\begin{array}{lr}\text { Data } & 8\end{array}$

2.1 Introduction . . . . . . . . . . . . . . . . . . 8 
2.2 Generalized Estimating Equations (GEEs) . . . . . . . . . . . 9

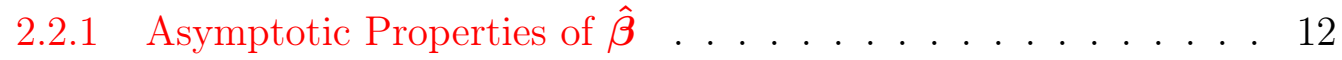

2.3 Second-Order Generalized Estimating Equations . . . . . . . . . . . . 13

2.3.1 Modeling the Correlation . . . . . . . . . . . . . . . 13

2.3.2 Approximate Variance of GEE Estimators . . . . . . . . . . 15

2.4 Application of GEE . . . . . . . . . . . . . . . . . . 15

2.4 .1 Case Studies . . . . . . . . . . . . . . . . . . . . . . . 16

2.4.1.1 Epileptic Seizures . . . . . . . . . . . 16

2.4.1.2 Clinical Trial of Antibiotics for Leprosy . . . . . . . 16

2.4.1.3 Data on Obesity from the Muscatine Coronary Risk Factor Study . . . . . . . . . . . . . . . . 17

3 Longitudinal Model for Missing Data $\quad 19$

3.1 Introduction . . . . . . . . . . . . . . . . . . . . 19

3.2 Missing Data Patterns in Longitudinal Study . . . . . . . . . . . . . 20

3.3 Weighted Generalized Estimating Equations . . . . . . . . . . . . . 22

3.3.1 Observation-Specific Weighted GEE . . . . . . . . . . . . 23

3.3.2 Subject-Specific Weighted GEE . . . . . . . . . . . . . 24

3.4 Incomplete Binary Longitudinal Model . . . . . . . . . . . . . . . . . 24

3.4.1 Logistic Response Model . . . . . . . . . . . . . . . . . . . . . 24

3.4.2 Dropout Model . . . . . . . . . . . . . . . . . . . 25

3.4.3 Methods of Estimation . . . . . . . . . . . . . . . 27

3.4.3.1 Generalized Estimating Equations . . . . . . . . 27

3.4.3.2 Weighted Generalized Estimating Equations . . . . . 28

3.4.3.3 Approximate Variance of WGEE Estimators . . . . . 30

3.4.4 Asymptotic Properties of WGEE Estimators . . . . . . . . . . 30

3.4.5 Simulation Study . . . . . . . . . . . . . . . 33

3.4.5.1 Response Models . . . . . . . . . . . . . . 34 
3.4.5.2 Dropout Models . . . . . . . . . . . . . . . 35

3.4.5.3 Estimating Dropout Probabilities . . . . . . . . 36

3.4.5.4 Assessment Methods . . . . . . . . . . . . 37

3.4.5.5 Results ................... 37

3.4.6 Application: Analysis of Smoking Data . . . . . . . . . . . . . 45

3.5 Incomplete Count Longitudinal Model . . . . . . . . . . . . . . . . . 48

3.5.1 Log Linear Response Model . . . . . . . . . . . . . . . . . . . 48

3.5.2 Methods of Estimation . . . . . . . . . . . . . . . . . 49

3.5.2.1 Generalized Estimating Equations . . . . . . . . 49

3.5.2.2 Weighted Generalized Estimating Equations . . . . . 50

3.5.2.3 Approximate Variance of WGEE Estimators . . . . . 51

3.5.3 Simulation Study . . . . . . . . . . . . . . 51

3.5.3.1 Generation of Correlated Counts . . . . . . . . . 52

3.5.3.2 Response Models . . . . . . . . . . . . . . 52

3.5.3.3 Dropout Models . . . . . . . . . . . . . . 52

3.5.3.4 Estimating Dropout Probabilities . . . . . . . . 53

3.5.3.5 Results .................. 54

3.6 Conclusion . . . . . . . . . . . . . . . . . . . 59

4 Semiparametric Longitudinal Model $\quad 60$

4.1 Introduction . . . . . . . . . . . . . . . . . . . . 60

4.2 Semiparametric Marginal Model . . . . . . . . . . . . . . . . . . 62

4.3 Regression Spline Methods . . . . . . . . . . . . . . . . . . 63

4.3.1 Selection of Number and Location of Knots . . . . . . . . . . 65

4.4 Partially Linear Binary Longitudinal Model . . . . . . . . . . . . . . 67

4.4 .1 Logistic Model . . . . . . . . . . . . . . . . . . . . . 67

4.4 .2 Method of Estimation . . . . . . . . . . . . . . 68

4.4.2.1 Generalized Estimating Equations . . . . . . . . 68 
4.4.2.2 Estimation Algorithm . . . . . . . . . . . 69

4.4.2.3 Computational Details for Estimating the Response Model .................. . . 71

4.4.3 Asymptotic Properties . . . . . . . . . . . . . . . . 72

4.4.3.1 Asymptotic for $\hat{m}(.) \ldots \ldots . \ldots . \ldots 72$

4.4 .4 Simulation Study . . . . . . . . . . . . . . . . 81

4.4.4.1 Response Models . . . . . . . . . . . . . . . . 81

4.4 .4 Results ................... 83

4.5 Partially Linear Count Longitudinal Model . . . . . . . . . . . . . . 84

4.5.1 Response Models . . . . . . . . . . . . . . . . 84

4.5.1.1 Log-Linear Model . . . . . . . . . . . . . . . . . 84

4.5.1.2 Partially Log-Linear Model . . . . . . . . . . . 85

4.5.2 Methods of Estimation . . . . . . . . . . . . . . 86

4.5.2.1 Generalized Estimating Equations . . . . . . . 86

4.5.2.2 Estimating Algorithm . . . . . . . . . . . 87

4.5.2.3 Computational Details for Estimating the Response

Model ................... 89

4.5.3 Simulation Study . . . . . . . . . . . . . . . . 89

4.5.3.1 Generation of Correlated Counts: . . . . . . . . . . 90

4.5.3.2 Response Models . . . . . . . . . . . . . . . 9 90

4.5.3.3 Results ...................... 91

4.6 Conclusions . . . . . . . . . . . . . . . . . . . . . . . . . 94

\section{Semiparametric Longitudinal Model With Missing Responses $\quad 95$}

5.1 Introduction . . . . . . . . . . . . . . . . . . 95

5.2 Partially Linear Model for Incomplete Binary Longitudinal Data . . . 96

5.2.1 Logistic Response Model . . . . . . . . . . . . . . . . 96

5.2 .2 Dropout Model . . . . . . . . . . . . . . . . . 97 
5.2 .3 Methods of Estimation . . . . . . . . . . . . . . . . . 98

5.2.3.1 Weighted Regression Spline Generalized Estimating Equations (WRSGEEs) . . . . . . . . . . . . 98

5.2.3.2 Estimating Dropout Probabilities . . . . . . . . . 100

5.2.3.3 Computational Details for Estimating the Response

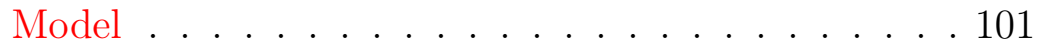

5.2.4 Asymptotic Properties . . . . . . . . . . . . . . . . . 102

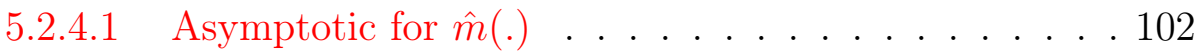

5.2.4.2 Proof of Theorem 2: . . . . . . . . . . . 103

5.2.5 Simulation Study . . . . . . . . . . . . . . . . 108

5.2.5.1 Response Models . . . . . . . . . . . . . . . . 108

5.2.5.2 Dropout Model . . . . . . . . . . . . . . 109

5.2.5.3 Results ................... . 110

5.2.6 Application: Analysis of Smoking Data . . . . . . . . . . . . 113

5.3 Partially Linear Model for Incomplete Count Longitudinal Data . . 118

5.3.1 Partially Log-Linear Response Model . . . . . . . . . . . . . . 118

5.3 .2 Methods of Estimation . . . . . . . . . . . . . 118

5.3.2.1 Weighted Regression Spline Generalized Estimating

Equations (WRSGEEs) . . . . . . . . . . 118

5.3.2.2 Estimating Dropout Probabilities . . . . . . . . 119

5.3.2.3 Computational Details for Estimating the Response Model . . . . . . . . . . . . . . . 120

5.3 .3 Simulation Study . . . . . . . . . . . . . . . . . . 121

5.3.3.1 Correlated Count Data . . . . . . . . . . . 121

5.3.3.2 Response Model . . . . . . . . . . . . . . . . . 122

5.3.3.3 Dropout Model . . . . . . . . . . . . . . . 123

5.3.3.4 Results ...................... 123 
6 Application: Health and Retirement Study (HRS)

6.1 Introduction . . . . . . . . . . . . . . . . . . 127

6.1.1 RAND HRS DATA Files Structure: . . . . . . . . . . . . . 128

6.1.2 Baseline Covariates . . . . . . . . . . . . . . . . . 129

6.1.3 Response Model . . . . . . . . . . . . . . . . . . . . . 132

6.1.4 Missing Data Model . . . . . . . . . . . . . . . . . 132

6.1.5 Estimation Methods . . . . . . . . . . . . . . 132

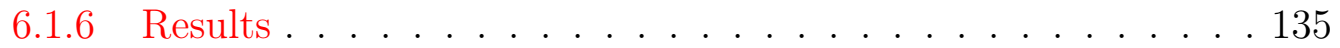

$\begin{array}{llr}7 & \text { Conclusion } & 139\end{array}$

7.1 Future Research . . . . . . . . . . . . . . . . . . . . . . 140

$\begin{array}{lr}\text { Bibliography } & 141\end{array}$

Appendix

A R Codes of Simulation Study for Correlated Binary Responses 149

$\begin{array}{ll}\text { B Additional Simulation Results } & 167\end{array}$

C Analysis of a subset of HRS data, with the maximum number of $\begin{array}{lr}\text { doctor visits of } 100 . & 169\end{array}$ 


\section{List of Abbreviations}

\begin{tabular}{|c|c|}
\hline MAR & Missing at Random \\
\hline MCAR & Missing Completely at Random \\
\hline NMAR & Not Missing at Random \\
\hline NI & Non-Ignorable \\
\hline GEE & Generalized Estimating Equation \\
\hline WGEE & Weighted Generalized Estimating Equation \\
\hline RSGEE & Regression Spline Generalized Estimating Equation \\
\hline WRSGEE & Weighted Regression Spline Generalized Estimating Equation \\
\hline $\mathrm{CI}$ & Confidence Interval \\
\hline GLM & Generalized Linear Model \\
\hline GLMM & Generalized Linear Mixed Model \\
\hline GPLMM & Generalized Partially Linear Mixed Model \\
\hline IRLS & Iteratively Reweighted Least Squares \\
\hline LM & Linear Model \\
\hline ML & Maximum Likelihood \\
\hline MLE & Maximum Likelihood Estimator \\
\hline MSE & Mean Squared Error \\
\hline
\end{tabular}




\section{List of Tables}

3.1 Empirical relative biases, mean squared errors and coverage probabilities of GEE, WGEE1 and WGEE2 estimators under MAR dropout. [True parameter values: $\boldsymbol{\beta}=(-1,1,0.2)^{\prime}, \alpha=0.5$ and $\boldsymbol{\tau}=(-2,3,0)^{\prime}$.]

3.2 Empirical relative biases, mean squared errors and coverage probabilities of GEE, WGEE1 and WGEE2 estimators under MAR dropout. [True parameter values: $\boldsymbol{\beta}=(-1,1,0.2)^{\prime}, \alpha=0.5$ and $\boldsymbol{\tau}=(-2,2,0)^{\prime}$.]

3.3 Empirical percentage relative biases, mean squared errors and coverage probabilities of GEE, WGEE1 and WGEE2 estimators under NI drop-out model.[True parameter values: $\boldsymbol{\beta}=(-1,1,0.2)^{\prime}, \alpha=0.5$ and $\tau=(-2,3,0.5)^{\prime}$.] $\ldots \ldots \ldots \ldots 4 \ldots \ldots \ldots$

3.4 Empirical percentage relative biases, mean squared errors and coverage probabilities of GEE, WGEE1 and WGEE2 estimators under NI drop-out model.[True parameter values: $\boldsymbol{\beta}=(-1,1,0.2)^{\prime}, \alpha=0.5$ and $\left.\tau=(-2,3,1)^{\prime}.\right] \ldots \ldots \ldots \ldots \ldots$

3.5 Percentage relative biases and mean squared errors of maximum likelihood estimates under MAR and nonignorible dropout models. . . . 44

3.6 ML estimates and standard errors of missing data model parameters for the CARDIA study. . . . . . . . . . . . . . . . . . . 47

3.7 Estimates and standard error of regression and association parameters for the CARDIA study. . . . . . . . . . . . . . . . . . 47 
3.8 Empirical percentage relative biases, mean squared errors (MSEs) and coverage probabilities of GEE, WGEE1 and WGEE2 estimators under correctly specified MAR dropout model. True parameter values: $\boldsymbol{\beta}=(1.60,-0.51,-0.50,-0.50)^{\prime}, \alpha=0.20, \phi=1.30$ and $\tau=(-1.5,0.3,0)^{\prime} \ldots \ldots \ldots \ldots \ldots \ldots$

3.9 Empirical percentage relative biases, mean squared errors (MSEs) and coverage probabilities of GEE, WGEE1 and WGEE2 estimators under misspecified NI dropout model. True parameter values: $\boldsymbol{\beta}=$ $(1.60,-0.51,-0.50,-0.50)^{\prime}, \alpha=0.20, \phi=1.30$ and $\tau=(-1.5,0.3,0.3)^{\prime} .57$

3.10 Empirical relative biases and mean squared errors of maximum likelihood estimates under MAR and nonignorible dropout models. . . . 58

4.1 Empirical biases and mean squared errors (MSEs) of ordinary GEE and proposed RSGEE estimators when $m(z)=\theta+\sin (\pi(1-z)) ; z=$ $0.03,0.32,0.55,0.77,0.99$.

4.2 Empirical biases and mean squared errors (MSEs) of ordinary GEE and proposed RSGEE estimators when $m(z)=\sin (8 * z) ; z=0,0.2,0.5,0.7,0.9 .83$

4.3 Biases and MSEs under Prentice's second-order GEE and proposed method RSGEE. True $m(z)=0.5+0.2 z \ldots \ldots . \ldots . \ldots 92$

4.4 Biases and MSEs under usual Prentice's second-order GEE and proposed RSGEE. True $m(z)=0.5+0.2 z /(0.05+z) \ldots \ldots . \ldots . . . .93$

5.1 Empirical biases and mean squared errors (MSEs) of GEE, RSGEE and WRSGEE estimators of regression and association parameters.

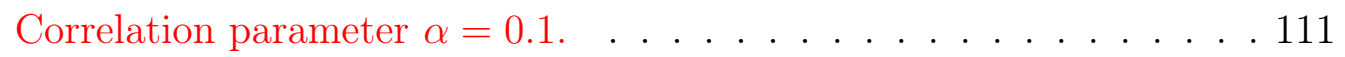

5.2 Empirical biases and mean squared errors (MSEs) of GEE, RSGEE and WRSGEE estimators of regression and association parameters.

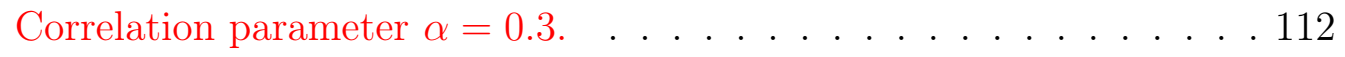

5.3 ML estimates and standard errors of missing data model parameters for the CARDIA study. . . . . . . . . . . . . . . . 116 
5.4 Estimates and standard error of regression and association parameters for the CARDIA study. . . . . . . . . . . . . . . 116

5.5 The estimated nonlinear function $m(z)$ at different of $z($ age/10) for CARDIA study. . . . . . . . . . . . . . . . . 117

5.6 Empirical biases and mean squared errors (MSEs) of GEE, RSGEE and WRSGEE estimators of regression and association parameters. . 124

5.7 Empirical biases and mean squared errors (MSEs) of GEE, RSGEE and WRSGEE estimators of regression and association parameters. . 125

6.1 Summary Statistics of RAND HRS Data . . . . . . . . . . . . . 131

6.2 ML estimates, standard errors and z-value of missing data model parameters for the HRS data analysis . . . . . . . . . . 136

6.3 Estimates, standard errors and z-values of regression, association and dispersion parameters for the HRS data analysis . . . . . . . . . . . 137

6.4 Estimates of nonlinear function $m$ (age) for different values of age . . 138

B.1 Empirical biases and mean squared errors (MSEs) of GEE, RSGEE and WRSGEE estimators of regression and association and dispersion parameters. . . . . . . . . . . . . . . . 168

C.1 ML estimates, standard errors and z-value of missing data model parameters for the HRS data analysis . . . . . . . . . . . . . 169

C.2 Estimates, standard error and z-value of regression, association and dispersion parameters for the HRS data analysis . . . . . . . . . . . 170

C.3 The nonlinear function $\mathrm{m}$ (Age) was estimated based on the spline function for different values of age . . . . . . . . . . . . . . 171 


\section{List of Figures}

4.1 Nonlinear functions, (a) $m(z)=1+\sin (\pi(1-z))$ and (b) $m(z)=$ $\sin (8 * z)$, as used in the simulation study. . . . . . . . . 82

$4.2 z$ vs. $m(z)$ for linear and nonlinear functions, as used in the simulation study . . . . . . . . . . . . . . . . . . . 91

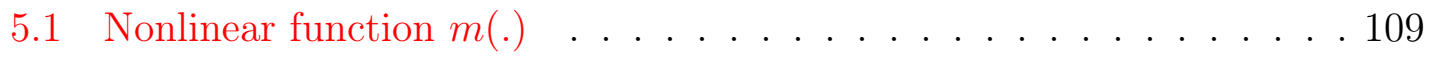

5.2 Estimated nonlinear function $\hat{m}($ age $)$ for the CARDIA study . . . . 115

6.1 (a) Scatter plot of mean visits vs. age. (b) Box plots of doctor visits. 130

6.2 Estimated nonlinear curve for HRS data analysis . . . . . . . . 133

6.3 Residual plots form HRS data analysis . . . . . . . . . . . . . . 134 


\section{Chapter 1}

\section{Introduction}

\subsection{Longitudinal Studies}

A longitudinal study refers to observational research in which a set of individuals or units are monitored repeatedly for a specified study period and data are recorded for each individual or unit. On the other hand, a study in which the data are collected from different subjects at the same time instead of different time points is called a cross-sectional study (Diggle et al., 2002).

Longitudinal studies allow researchers to investigate the change in a response variable over time along with the possibility of changes in some covariates. Also, unlike cross-sectional studies, longitudinal studies can capture the within-subject change. Interdependence among the observations that are taken from similar individuals is one of the main features of longitudinal studies. The observations which are taken from the same individual may be considered as the same cluster. Longitudinal data can be considered as clustered data with the main difference in that in clustered data, observations need not require any order, while in longitudinal data observations must have a sequential order.

The two important types of longitudinal models are marginal and mixed effects models. Marginal models capture the averaged mean structure of the population while, on the other hand, mixed effects models capture the conditional mean struc- 
ture of the population. A longitudinal study has an important role in clinical research, epidemiology and medical assessment. The study of longitudinal data is used to characterize aging and normal growth, and measures the effects of risk factors on human health and also assesses the effectiveness of treatments.

Longitudinal data can be collected either retrospectively, by digging up several measurements from historic records on each person, or prospectively, by following subjects forward in time. But commonly these are collected prospectively since repeated measurement quality collected from previous records or from individuals' memory may be poor. We usually collect longitudinal data in medical, environmental and biological studies, where measurements from similar subjects are repeatedly taken over a specific period of time.

In longitudinal studies, a common goal is to explain the variation in the response variable over time together with the change in variables that are responsible. The application of proper statistical methods is required for analyzing longitudinal data because of intercorrelations within subjects. The correlations must be taken into account to draw good scientific inferences. Longitudinal data analysis is simple because different subjects are assumed independent. Meaningful interpretations can be made by borrowing strength across the subjects. That is, the stability of a pattern across different subjects is the root of essential conclusions. For this reason, inferences on model assumptions for longitudinal data are more robust than those for time-series data, mainly the assumptions about correlations (Diggle et al., 2002).

\subsection{Dropouts in Longitudinal Data}

The most challenging and common problem of longitudinal studies is the presence of missing observations in the data. When observations are missing, the data are essentially uneven over time since all the individuals do not have the same number of repeated measurements taken from the same collection of events. Longitudinal data are defined as complete and balanced when there is no missing data and all individuals are measured at the same set of events (Fitzmaurice et al., 2012). 
We rely on conditional variables for studying dropouts in longitudinal outcomes. When data are missing, the standard GEE approaches of Liang and Zeger (1986) and Prentice (1988) are only valid where data are missing completely at random (MCAR) (Robins et al., 1995); i.e., given the covariates, the missing data process is independent of both the observed and unobserved data. The standard GEE estimator may be biased under a weaker assumption of missing at random (MAR), in which missingness depends on the observed findings but not on the unobserved values (Fitzmaurice et al., 1995).

\subsection{Marginal Models for Longitudinal Data}

In the case of a discrete longitudinal response, linear models are not suitable for connecting variations in the mean response to covariates. As a result, statisticians have established new additions of generalized linear models (GLMs) for longitudinal data. In the analysis of regression, generalized linear models make available cohesive categories of models of independent observations for continuous and discrete responses.

In GLMs, an appropriate nonlinear conversion of the mean response is supposed to be a linear covariates function. Statisticians extended the study of GLMs simply to handle the observations of longitudinal data in several different ways. The three extensive but different categories of regression models for longitudinal data are:

1. Population-averaged models or marginal models,

2. Subject-specific models or random-effects models, and

3. Response conditional models or transition models.

These models not only vary in the way the association between repetitive measures is compensated for, but also include regression parameters with distinctly specific definitions. Fitzmaurice et al. (2008) pointed out that these alternatives in analysis reflect the changed targets of the conclusion of these models. The models considered in this thesis are marginal or population-averaged. 
Population-averaged models or marginal models are a class of regression models in which generalized linear models can be extended to longitudinal data. The word marginal in this perspective is used to highlight that the mean response model at each event just depends on covariates, and does not depend on any earlier responses or random effects. In mixed-effects models, the mean response is not only dependent on covariates, but also on the vector of random effects, and in transitional or response conditional models, the mean response also depends on earlier responses.

Marginal models make available a direct method to extend generalized linear models to longitudinal data. By using a suitable link function, these directly model the mean response on every event. According to Fitzmaurice et al. (2008), marginal models have the emphasis on the marginal mean of the response variable and its dependencies on the covariates. The marginal models do not essentially need complete assumptions on the distribution of the vector of responses, but just a regression model for the mean response.

\subsection{Semiparametric Models for Longitudinal Data}

The relation between the covariates and response may be complicated and linear expressions may not be sufficient to form the association. Grace et al. (2009) pointed out that it would be impractical and worse by fitting a linear model into a nonlinear type of association. Heckman (1986), Shi et al. (1996), Carroll et al. (1997), Lin and Ying (2001), Wu and Zhang (2002), Fan and Li (2004), Wang (2003), Wang et al. (2005), Lin et al. (2004), He et al. (2005), Huang et al. (2007), and Leng et al. (2010) among many others studied essential current interests in emerging semiparametric and nonparametric regression techniques for clustered or longitudinal types of data.

The partially linear model resolves dimensionality problems by supposing certain parametric forms. The outcome variable mean in a partially linear model is supposed to rely on certain $\mathbf{X}$ covariates parametrically and some $\mathbf{Z}$ covariates nonparametrically. This model is mainly attractive when effects of $\mathbf{X}$ are of key interest, however, confounding effects of $\mathbf{Z}$ are nuisance parameters. These models are generalized 
grouping of simpler models, that is, partially linear models and single index models, often referred to as generalized partially linear models (GPLM), and generalized partially linear single-index models (GPLSIM) as used in Carroll et al. (1997).

Efficient estimation for partially linear models is broadly studied for independent data; see, for example, Speckman (1988), Severini and Staniswalis (1994). In the sense of the Cox regression model for survival data, Martinussen and Scheike (2002) considered a partially linear model. For a partially linear model with clustered data, the main concern is to efficiently examine within cluster correlation.

According to Lin and Carroll (2001a), when the parametric covariates rely on nonparametric covariates, an expected use of the local kernel polynomial technique cannot suitably account for within cluster correlation, thus fails to produce an effectual semiparametric estimator. Conversely, the similar kind of kernel technique can account for within cluster correlation and creates an effectual estimator in case of a nonparametric covariate model (Lin and Carroll, 2001b). Fan and Li (2004) considered the profile local linear approach and the joint modelling for partially linear models.

GEE methods for analyzing semiparametric partially linear models and their numerous extensions turn out to be more and more common (see, for example, Fan and Li, 2004; He et al., 2002 and Qin et al., 2016). Most of the research, however, concentrated on estimating only the marginal mean parameters and did not consider the joint estimation of the marginal mean, association and dispersion parameters.

\subsection{Statement of the Problems}

Partially linear marginal models for binary and count longitudinal data with dropouts are suggested and explored in this thesis. In the case of the partially linear marginal model, the key subject of our attention is the estimation of joint marginal mean, association and dispersion parameters through generalized secondorder estimating equations. These problems are explored in three sections as follows. 
In the first section of the study (Chapter 3), a group of weighted generalized estimating equations (GEEs) for binary and count longitudinal responses with dropouts are investigated. The suggested technique produces unbiased estimators of regression and association parameters as well as parameters of missing data models. Simulations were carried out to examine the properties of the proposed estimators under both misspecified and correctly specified correlation patterns. The suggested technique is also applied to a real incomplete longitudinal data set on cigarette smoking trends in young adults to examine the coronary artery development.

In the second section of the study (Chapter 4), a semiparametric technique derived from second-order GEEs for binary and count longitudinal responses are explored. For the estimation of the nonparametric section of the model, a smoothing technique based on regression spline is used. The empirical properties of the proposed method are studied using simulations.

In the last section of this study (Chapter 5), we suggest a weighted secondorder GEE method to simultaneously estimate the nonlinear function, regression, association and dispersion parameters for partially linear dropout models. The implementation of the suggested technique is also done on the Health and Retirement Study. In this study, we are expanding the proposed semiparametric approach to analyze incomplete count longitudinal data with dropouts. The number of visits with a medical doctor in the last two years by a respondent was considered as the response variable. Roughly, $20 \%$ patients have missing observations due to dropouts. This study aims to recognize subgroups of respondents with similar behaviours in terms of the number of visits to a doctor's office and to clarify what predictors affect the number of visits and how.

\subsection{Organization of Thesis}

The thesis is organized as follows. In Chapter 2, we review ordinary GEE approaches from the perspective of their use in longitudinal studies. 
We introduce the proposed methodology and estimation algorithm based on the weighted GEE for fitting regression models to incomplete binary and count longitudinal responses in Chapter 3. We also present results from simulations and real data analysis in Chapter 3.

In Chapter 4, we study the proposed methodology based on the second-order GEE approach for analyzing longitudinal binary and count responses under partially linear models. We also present the results from simulation studies.

We propose the weighted second-order GEE method for the simultaneous estimation of the nonlinear function, regression, association and dispersion parameters for partially linear models with dropouts in Chapter 5. We study the empirical properties of the proposed method in simulations.

Chapter 6 presents the implementation of the proposed methods using actual data from the Health and Retirement Study (HRS)(HRS, 2019). The thesis is concluded in Chapter 7 with some discussion and direction to further studies. 


\section{Chapter 2}

\section{Overview of Generalized Estimating Equations for Longitudinal Data}

The objective of this chapter is to review methods of generalized estimating equations (GEEs) introduced by Liang and Zeger (1986) and the GEE2 approach of Prentice (1988), which are widely used for analyzing binary and count longitudinal data.

\subsection{Introduction}

Generalized estimating equations (GEEs) are commonly used for estimating parameters of marginal models for longitudinal data. These are a class of estimating equations introduced by Liang and Zeger (1986) that give reliable estimators of regression parameters under mild assumptions about the time dependence, without identifying the actual distribution of the longitudinal measurements.

The technique of GEE fits generalized linear models (GLMs) by using generalized estimating equations for longitudinal data and is a multivariate analog of the quasilikelihood. This technique fits marginal models to longitudinal data and is frequently used to evaluate longitudinal data whenever the average effect of the population is of main interest (Liang and Zeger, 1986). The technique of GEE is grounded on a structure of "working" correlation, and even in a misspecified structure of correlation 
this provides consistent estimators.

The first-order GEE approach is attractive in that it does not need detailed requirements of the combined distribution for a longitudinal response; it is based only on the first two moments. Although, most frequently the interest is to perform the analysis by considering the parameters of the regression model, in recent years, there has been a growing interest in the association parameters. Yi and Cook (2002) point out that when parameters of association is of main importance, GEEs of second-order can be used for an effective evaluation. The approach of GEE can be extended for estimating both association and regression parameters of marginal models by Prentice (1988). Fitzmaurice et al. (1995), Lipsitz et al. (1991) and Carey et al. (1993) studied longitudinal data by forming an association between repeated responses and modeling the association among responses in terms of pairwise odds ratios.

\subsection{Generalized Estimating Equations (GEEs)}

The GEE is a class of estimating equations that takes the correlation into account to increase the efficiency of the estimators of regression parameters $\boldsymbol{\beta}$. To illustrate this, we first introduce some notation that will be used throughout the thesis.

Let $y$ be the response variable and $\mathbf{x}$ the vector of covariates. We denote fixed effects parameters by $\boldsymbol{\beta}$. The general form of our model is $E(y \mid \mathbf{x})=f^{-1}\left(\mathbf{x}^{\prime} \boldsymbol{\beta}\right)$, where $f^{-1}(\cdot)$ is a given function (identity function in case of linear models or an inverse of a suitable link function in generalized linear models). Let $y_{i t}$ denote the response variable from the $i^{\text {th }}$ subject/cluster on the $t^{\text {th }}$ measurement occasion, where $1 \leq i \leq k$ and $1 \leq t \leq T$, and $\mathbf{y}_{i}=\left(y_{i 1}, y_{i 2}, \ldots, y_{i T}\right)^{\prime}$ be the vector of all observations from subject/cluster $i$. Each individual also has a $p \times 1$ covariate vector $\mathbf{x}_{i t}=\left(x_{i t 1}, \ldots, x_{i t p}\right)^{\prime}$ measured at time $\mathrm{t}$, which include both time stationary and time varying covariates. The $T \times p$ design matrix of all covariates associated with subject $i$ is denoted by $\mathbf{X}_{i}=\left(\mathbf{x}_{i 1}, \ldots, \mathbf{x}_{i T}\right)^{\prime}$ and $\mathbf{Y}=\left(\mathbf{y}_{1}^{\prime}, \mathbf{y}_{2}^{\prime}, \ldots, \mathbf{y}_{k}^{\prime}\right)^{\prime}$ represents the vector of all outcomes in the study. The three important parts of the marginal 
model for longitudinal data are:

1. Marginal mean response $E\left(y_{i t} \mid \mathbf{x}_{i t}\right)=\mu_{i t}$ depends on covariates through a known link function

$$
f\left(\mu_{i t}\right)=\eta_{i t}=\mathbf{x}_{i t}^{\prime} \boldsymbol{\beta}
$$

2. Marginal variance of $y_{i t}$, given the covariates, is related to the marginal mean by

$$
\operatorname{var}\left(y_{i t} \mid \mathbf{x}_{i t}\right)=\phi v\left(\mu_{i t}\right)
$$

where $v\left(\mu_{i t}\right)$ is a known variance function, $\phi$ is a scale parameter that may be known or need to be estimated. For instance, in case of continuous response, $\phi$ is a scale parameter that must be estimated, whereas for a binary response, $\phi$ may be fixed at 1 . In the case of a count data, $\phi$ is frequently estimated from the data at hand to accommodate overdispersion in Poisson regression.

3. The pairwise within-subject relationship amongst the vector of repeated responses, when covariates are given, is often considered as an additional set of association parameters. For example, when parameter vector $\boldsymbol{\alpha}$ represents the pairwise correlations amongst the responses, the covariance between the responses rely on $\mu_{i t}\left(\mathbf{x}_{i t}^{\prime} \boldsymbol{\beta}\right), \phi$ and $\boldsymbol{\alpha}$. The matrix of covariance is built by the multiplication of standard deviations and correlations as

$$
\mathbf{V}_{i}=\phi \mathbf{A}_{i}^{\frac{1}{2}} \boldsymbol{\rho}(\boldsymbol{\alpha}) \mathbf{A}_{i}^{\frac{1}{2}}
$$

where $\mathbf{A}_{i}$ is a diagonal matrix with diagonal elements $v\left(\mu_{i t}\right)$ and $\boldsymbol{\rho}(\boldsymbol{\alpha})$ is the correlation matrix (function of $\boldsymbol{\alpha}$ ).

The GEE estimator of $\boldsymbol{\beta}$ for given $(\boldsymbol{\alpha}, \phi)$ introduced by Liang and Zeger (1986) is found by solving

$$
\begin{aligned}
\mathbf{U}_{\boldsymbol{\beta}}(\boldsymbol{\beta}, \boldsymbol{\alpha}, \phi) & =\sum_{i=1}^{k} \mathbf{U}_{\boldsymbol{\beta}, i}=\sum_{i=1}^{k} \mathbf{D}_{i}^{\prime} \mathbf{V}_{i}^{-1}\left(\mathbf{y}_{i}-\boldsymbol{\mu}_{i}(\boldsymbol{\beta})\right) \\
& =\sum_{i=1}^{k} \mathbf{X}_{i}^{\prime} \boldsymbol{\Delta}_{i} \mathbf{V}_{i}^{-1}\left(\mathbf{y}_{i}-\boldsymbol{\mu}_{i}(\boldsymbol{\beta})\right)=0
\end{aligned}
$$


where $\mathbf{D}_{i}=\left(\partial \boldsymbol{\mu}_{i} / \partial \boldsymbol{\beta}\right)=\left(\partial \boldsymbol{\mu}_{i} / \partial \boldsymbol{\eta}_{i}\right)\left(\partial \boldsymbol{\eta}_{i} / \partial \boldsymbol{\beta}\right)=\boldsymbol{\Delta}_{i} \mathbf{X}_{i}$, with $\boldsymbol{\Delta}_{i}\left(\boldsymbol{\mu}_{i}(\boldsymbol{\beta})\right)=\left(\partial \boldsymbol{\mu}_{i} \mid \partial \boldsymbol{\eta}_{i}\right)=$ $\operatorname{diag}\left\{\dot{\mu}_{i 1}(\boldsymbol{\beta}), \ldots, \dot{\mu}_{i T}(\boldsymbol{\beta})\right\}$, with $\dot{\mu}($.$) is the inverse function derivative of \mu()=$. $f^{-1}(),. \mathbf{V}_{i}(\boldsymbol{\beta}, \boldsymbol{\alpha}, \phi)=\phi \mathbf{A}_{i}^{1 / 2} \boldsymbol{\rho}(\boldsymbol{\alpha}) \mathbf{A}_{i}^{1 / 2}$, with $\mathbf{A}_{i}=\operatorname{diag}\left\{v\left(\mu_{i 1}\right), \ldots, v\left(\mu_{i T}\right)\right\}$, while $\boldsymbol{\rho}(\boldsymbol{\alpha})$ is a working correlation matrix for $\mathbf{y}_{i}$, depending on the vector of association parameters $\boldsymbol{\alpha}$.

There are different types of working correlation structures. For example, independent correlation structure:

$$
\operatorname{Corr}\left(y_{i t}, y_{i t^{\prime}}\right)=\left\{\begin{array}{cc}
1 & \text { if } t=t^{\prime} \\
0 & \text { if } t \neq t^{\prime}
\end{array}, \quad \boldsymbol{\rho}(\boldsymbol{\alpha})=\left(\begin{array}{lll}
1 & 0 & 0 \\
0 & 1 & 0 \\
0 & 0 & 1
\end{array}\right)_{3 \times 3}\right.
$$

Exchangeable correlation structure:

$$
\operatorname{Corr}\left(y_{i t}, y_{i t^{\prime}}\right)=\left\{\begin{array}{cc}
1 & \text { if } t=t^{\prime} \\
\alpha & \text { if } t \neq t^{\prime}
\end{array}, \quad \boldsymbol{\rho}(\boldsymbol{\alpha})=\left(\begin{array}{ccc}
1 & \alpha & \alpha \\
\alpha & 1 & \alpha \\
\alpha & \alpha & 1
\end{array}\right)_{3 \times 3}\right.
$$

Serial correlation structure:

$$
\operatorname{Corr}\left(y_{i t}, y_{i t^{\prime}}\right)=\left\{\begin{array}{ll}
1 & \text { if } t=t^{\prime} \\
\alpha^{\left|t-t^{\prime}\right|} & \text { if } t \neq t^{\prime}
\end{array}, \quad \boldsymbol{\rho}(\boldsymbol{\alpha})=\left(\begin{array}{ccc}
1 & \alpha & \alpha^{2} \\
\alpha & 1 & \alpha \\
\alpha^{2} & \alpha & 1
\end{array}\right)_{3 \times 3}\right.
$$

Unstructured correlation structure:

$$
\operatorname{Corr}\left(y_{i t}, y_{i t^{\prime}}\right)=\left\{\begin{array}{cc}
1 & \text { if } t=t^{\prime} \\
\alpha_{t t^{\prime}} & \text { if } t \neq t^{\prime}
\end{array}, \quad \boldsymbol{\rho}(\boldsymbol{\alpha})=\left(\begin{array}{ccc}
1 & \alpha_{12} & \alpha_{13} \\
\alpha_{12} & 1 & \alpha_{23} \\
\alpha_{13} & \alpha_{23} & 1
\end{array}\right)_{3 \times 3}\right.
$$

By using an iterative method, the above estimating equations (2.1) for $\boldsymbol{\beta}$ can be solved numerically. If $\boldsymbol{\alpha}$ and $\phi$ are known, the GEE method provides estimate $\hat{\boldsymbol{\beta}}$ 
that is asymptotically efficient (Liang and Zeger, 1986).

Liang and Zeger (1986) also reflected the estimation of association parameters $\boldsymbol{\alpha}$ by method of moments based on Pearson residuals,

$$
\hat{r}_{i t}=\frac{\left(y_{i t}-\hat{\mu}_{i t}\right)}{\sqrt{v\left(\hat{\mu}_{i t}\right)}}
$$

The moment estimators $\hat{\alpha}_{t t^{\prime}}$ are obtained as

$$
\hat{\alpha}_{t t^{\prime}}=\sum_{i=1}^{k} \hat{r}_{i t} \hat{r}_{i t}^{\prime} /(k-p)
$$

and the dispersion parameter $\phi$ can be estimated by

$$
\hat{\phi}=\sum_{i=1}^{k} \sum_{t=1}^{T} \hat{r}_{i t}^{2} /(N-p)
$$

where $N=k \times T$ is the total number of observations. The solution $\hat{\boldsymbol{\beta}}$ to $(2.1)$ for given $(\boldsymbol{\alpha}, \phi)$ is obtained by iteratively re-weighted least squares, which yields consistent estimates of $\boldsymbol{\beta}$.

\subsubsection{Asymptotic Properties of $\hat{\boldsymbol{\beta}}$}

For given $\boldsymbol{\alpha}$ and $\phi$, Liang and Zeger (1986) showed that $k^{1 / 2}(\hat{\boldsymbol{\beta}}-\boldsymbol{\beta})$ is asymptotically distributed as multivariate normal with mean zero and covariance matrix $\mathbf{V}(\boldsymbol{\beta})=\lim _{k \rightarrow \infty} k \mathbf{H}_{\boldsymbol{\beta}}^{-1} \mathbf{O}_{\boldsymbol{\beta}} \mathbf{H}_{\boldsymbol{\beta}}^{-1}$, where $\mathbf{H}_{\boldsymbol{\beta}}=\sum_{i=1}^{k} \mathbf{D}_{i}^{\prime} \mathbf{V}_{i}^{-1} \mathbf{D}_{i}$ and $\mathbf{O}_{\boldsymbol{\beta}}=\sum_{i=1}^{k} \mathbf{D}_{i}^{\prime} \mathbf{V}_{i}^{-1}$ $\operatorname{Cov}\left(\mathbf{y}_{i}\right) \mathbf{V}_{i}^{-1} \mathbf{D}_{i}$. The estimator $\hat{\boldsymbol{\beta}}$ is asymptotically normal and consistent under weak situations even when $(\boldsymbol{\alpha}, \phi)$ are substituted by any $k^{1 / 2}-$ consistent estimators.

The proposed variance estimate of $\hat{\boldsymbol{\beta}}$ by Liang and Zeger (1986) is

$$
\mathbf{V}(\hat{\boldsymbol{\beta}})=\sum_{i=1}^{k}\left(\hat{\mathbf{D}}_{i}^{\prime} \hat{\mathbf{V}}_{i}^{-1} \hat{\mathbf{D}}_{i}\right)^{-1}\left\{\sum_{i=1}^{k} \hat{\mathbf{D}}_{i}^{\prime} \hat{\mathbf{V}}_{i}^{-1} \mathbf{C o v}\left(\hat{\mathbf{y}}_{i}\right) \hat{\mathbf{V}}_{i}^{-1} \hat{\mathbf{D}}_{i}\right\} \sum_{i=1}^{k}\left(\hat{\mathbf{D}}_{i}^{\prime} \hat{\mathbf{V}}_{i}^{-1} \hat{\mathbf{D}}_{i}\right)^{-1},
$$

where a hat $\widehat{()}$ indicates calculation at $\hat{\boldsymbol{\beta}}, \hat{\boldsymbol{\alpha}}$, and $\operatorname{Cov}\left(\hat{\mathbf{y}}_{i}\right)=\left(\mathbf{y}_{i}-\hat{\boldsymbol{\mu}}_{i}\right)\left(\mathbf{y}_{i}-\hat{\boldsymbol{\mu}}_{i}\right)^{\prime}$. The asymptotic efficiency of $\hat{\boldsymbol{\beta}}$ is likely to be near to unity if the form of the work- 
ing correlation matrix is almost correct. Next, an extension of the GEE approach to the joint estimation of the pairwise correlation and marginal mean response is considered.

\subsection{Second-Order Generalized Estimating Equations}

\subsubsection{Modeling the Correlation}

Prentice (1988) extended the GEE technique to allow simultaneous estimation of the vector of regression parameters $\boldsymbol{\beta}$ and association parameters $\boldsymbol{\alpha}$, which characterize the marginal pairwise correlations. This approach is known as GEE2 because it is a second-order extension of the GEE approach developed by Liang and Zeger (1986). Even if the interest lies in the marginal probabilities of the response, careful modelling of the correlation can improve the efficiency of the regression estimators (Prentice, 1988). From a second set of estimating equations, a GEE estimator of the correlation parameter $\boldsymbol{\alpha}$ can be obtained.

Specifically, for estimating $\boldsymbol{\alpha}$, Prentice (1988) suggests solving the equations

$$
\mathbf{U}_{\boldsymbol{\alpha}}(\boldsymbol{\beta}, \boldsymbol{\alpha})=\sum_{i=1}^{k} \mathbf{L}_{i}^{\prime} \mathbf{W}_{i}^{-1}\left(\mathbf{r}_{i}-\boldsymbol{\delta}_{i}(\boldsymbol{\alpha})\right)=0
$$

where $\mathbf{r}_{i}=\left(r_{i 1} r_{i 2}, r_{i 1} r_{i 3}, \ldots, r_{i 1} r_{i T}, r_{i 2} r_{i 3}, \ldots, r_{i, T-1} r_{i T}, r_{i 1}^{2}, r_{i 2}^{2}, \ldots, r_{i T}^{2}\right)^{\prime}$ comprises the products of subject $i$ 's pairs and squares of Pearson residuals

$$
r_{i t}=\frac{\left(y_{i t}-\mu_{i t}\right)}{\sqrt{v\left(\mu_{i t}\right)}}
$$

To find the variance of $\mathbf{r}_{i}$, we can show

$$
\begin{aligned}
E\left(r_{i t}^{2} r_{i u}^{2}\right) & =\left[E\left(y_{i t}^{2} y_{i u}^{2}\right)-2 \mu_{i t} E\left(y_{i t}^{2} y_{i u}\right)+\mu_{i t}^{2} E\left(y_{i u}^{2}\right)-2 \mu_{i t} E\left(y_{i t} y_{i u}^{2}\right)\right. \\
& \left.+4 \mu_{i t} \mu_{i u} E\left(y_{i t} y_{i u}\right)-3 \mu_{i t}^{2} \mu_{i u}^{2}+\mu_{i u}^{2} E\left(y_{i t}^{2}\right)\right]\left[v\left(\mu_{i t}\right) v\left(\mu_{i u}\right]^{-1}\right.
\end{aligned}
$$

for $t<u<T, \boldsymbol{\delta}_{i}=E\left(\mathbf{r}_{i}\right), \mathbf{L}_{i}=\partial \boldsymbol{\delta}_{i} / \partial \boldsymbol{\alpha}$, and $\mathbf{W}_{i}=\operatorname{var}\left(\mathbf{r}_{i}\right)$. The choice of the 
weighted matrix, $\mathbf{W}_{i}$, depends on the type of the responses. In the case of the binary response, the last $T$ components of $\mathbf{r}_{i}$ may be skipped, but this is not the case when the response is count, as in count responses, we have $T^{*}=\left(\begin{array}{l}T \\ 2\end{array}\right)+T$ elements of $\mathbf{r}_{i}$. There are different types of $\mathbf{W}_{i}$ available in the literature; see Hall and Severini 1998 for more details. But we will use the identity matrix for $\mathbf{W}_{i}$, especially for count response when solving $\mathbf{U}_{\boldsymbol{\alpha}}(\boldsymbol{\beta}, \boldsymbol{\alpha})=0$.

Following Prentice (1988), estimators of $(\boldsymbol{\beta}, \boldsymbol{\alpha})$ can be obtained by solving the estimating equations

$$
\begin{aligned}
& \mathbf{U}_{\boldsymbol{\beta}}(\boldsymbol{\beta}, \boldsymbol{\alpha})=\sum_{i=1}^{k} \mathbf{D}_{i}^{\prime} \mathbf{V}_{i}^{-1}\left(\mathbf{y}_{i}-\boldsymbol{\mu}_{i}(\boldsymbol{\beta})\right)=0, \\
& \mathbf{U}_{\boldsymbol{\alpha}}(\boldsymbol{\beta}, \boldsymbol{\alpha})=\sum_{i=1}^{k} \mathbf{L}_{i}^{\prime} \mathbf{W}_{i}^{-1}\left(\mathbf{r}_{i}-\boldsymbol{\delta}_{i}(\boldsymbol{\alpha})\right)=0 .
\end{aligned}
$$

A simple iterative mechanism for calculating $(\hat{\boldsymbol{\beta}}, \hat{\boldsymbol{\alpha}})$ initiates with preliminary values $\left(\boldsymbol{\beta}_{0}, \boldsymbol{\alpha}_{0}\right)$ and yields efficient values $\left(\boldsymbol{\beta}_{m+1}, \boldsymbol{\alpha}_{m+1}\right)$ from interim values (initial values) $\left(\boldsymbol{\beta}_{m}, \boldsymbol{\alpha}_{m}\right)$ by means of

$$
\begin{aligned}
& \boldsymbol{\beta}_{m+1}=\boldsymbol{\beta}_{m}+\left(\sum_{i=1}^{k} \mathbf{D}_{i}^{\prime} \mathbf{V}_{i}^{-1} \mathbf{D}_{i}\right)^{-1} \sum_{i=1}^{k} \mathbf{D}_{i}^{\prime} \mathbf{V}_{i}^{-1}\left(\mathbf{y}_{i}-\boldsymbol{\mu}_{i}(\boldsymbol{\beta})\right), \\
& \boldsymbol{\alpha}_{m+1}=\boldsymbol{\alpha}_{m}+\left(\sum_{i=1}^{k} \mathbf{L}_{i}^{\prime} \mathbf{W}_{i}^{-1} \mathbf{L}_{i}\right)^{-1} \sum_{i=1}^{k} \mathbf{L}_{i}^{\prime} \mathbf{W}_{i}^{-1}\left(\mathbf{r}_{i}-\boldsymbol{\delta}_{i}(\boldsymbol{\alpha})\right) .
\end{aligned}
$$

The equations $\mathbf{U}_{\boldsymbol{\beta}}(\boldsymbol{\beta}, \boldsymbol{\alpha})=0$ and $E\left(\mathbf{y}_{i} \mid \mathbf{X}_{i}, \boldsymbol{\beta}\right)=\boldsymbol{\mu}_{i}$ at true $\boldsymbol{\beta}$ are unbiased under the mechanism of missing completely at random (MCAR)(Robins et al., 1995). But in the case of non-ignorable (NI) or missing at random (MAR) processes $E\left(\mathbf{y}_{i} \mid \mathbf{X}_{i}, \boldsymbol{\beta}\right) \neq$ $\boldsymbol{\mu}_{i}$ in general; so $\hat{\boldsymbol{\beta}}$ may not be consistent in such cases. 


\subsubsection{Approximate Variance of GEE Estimators}

The variance of the GEE estimators $\hat{\boldsymbol{\beta}}$ and $\hat{\boldsymbol{\alpha}}$ can be approximated by using sandwich type estimators (White, 1982). The variance of $\hat{\boldsymbol{\beta}}$ can be approximated as

$$
\mathbf{V}(\hat{\boldsymbol{\beta}})=\mathbf{H}_{\boldsymbol{\beta}}^{-1} \mathbf{O}_{\beta} \mathbf{H}_{\boldsymbol{\beta}}^{-1},
$$

where $\mathbf{H}_{\boldsymbol{\beta}}=\sum_{i=1}^{k} \mathbf{D}_{i}^{\prime} \mathbf{V}_{i}^{-1} \mathbf{D}_{i}$ and $\mathbf{O}_{\boldsymbol{\beta}}=\sum_{i=1}^{k} \mathbf{S}_{\boldsymbol{\beta}, i} \mathbf{S}_{\boldsymbol{\beta}, i}^{\prime}$, with $\mathbf{S}_{\boldsymbol{\beta}, i}=\mathbf{D}_{i}^{\prime} \mathbf{V}_{i}^{-1}\left(\mathbf{y}_{i}-\boldsymbol{\mu}_{i}\right)$.

Similarly, the variance of the GEE estimator $\hat{\boldsymbol{\alpha}}$ can be obtained as

$$
\mathrm{V}(\hat{\alpha})=\mathbf{H}_{\alpha}^{-1} \mathbf{O}_{\alpha} \mathbf{H}_{\alpha}^{-1}
$$

where $\mathbf{H}_{\boldsymbol{\alpha}}=\sum_{i=1}^{k} \mathbf{L}_{i}^{\prime} \mathbf{W}_{i}^{-1} \mathbf{L}_{i}$ and $\mathbf{O}_{\boldsymbol{\alpha}}=\sum_{i=1}^{k} \mathbf{S}_{\boldsymbol{\alpha}, i} \mathbf{S}_{\boldsymbol{\alpha}, i}^{\prime}$, with $\mathbf{S}_{\boldsymbol{\alpha}, i}=\mathbf{L}_{i}^{\prime} \mathbf{W}_{i}^{-1}\left(\mathbf{r}_{i}-\boldsymbol{\delta}_{i}\right)$.

The matrices $\mathbf{H}_{\boldsymbol{\alpha}}, \mathbf{H}_{\boldsymbol{\beta}}$ and $\mathbf{O}_{\boldsymbol{\alpha}}, \mathbf{O}_{\boldsymbol{\beta}}$ are evaluated at the GEE estimators $\hat{\boldsymbol{\alpha}}$ and $\hat{\boldsymbol{\beta}}$.

\subsection{Application of GEE}

GEE methods can be applied in numerous fields, especially in epidemiological and cohort studies. The epidemiological research investigates the dissemination and patterns of specific groups of health accidents, safety features, and their consequences or implications. In a cohort study, participants are monitored over time from weeks to years, based on age. The goal is to understand the relationship between some cohort-related features and the eventual outcome. One can use the GEE to analyze repeated categorical (e.g., binary and count data) responses, where the interest is focused primarily on the regression parameters for the marginal expectations of the categorical response. A model is extended by GEE methods to recurrent, linked and clustered categorical responses. 


\subsubsection{Case Studies}

In this section, we illustrate three different case studies, where the method of GEE is implemented on marginal models for analyzing longitudinal data.

\subsubsection{Epileptic Seizures}

The data are from a placebo-controlled clinical trial of 59 epileptics carried out by Mirelesh and Leppik (1985), where a randomized controlled clinical trial of the nonepileptic medication, progabide, studied people with partial seizures. As an adjuvant of regular anti-epileptic treatment, participants in the study were assigned to either progabide or placebo. Progabide is an anti-epileptic medication whose main action function is to increase the production of gamma-aminobutyric acid (GABA); GABA is the predominant inhibitory neurotransmitter within the brain. Baseline details on the number of epileptic seizures were reported during the intervening 8-week period before medication was obtained. The counts of epileptic seizures at 2-week periods were registered before each of four consecutive post-randomization clinic visits. The main aim of this research was to evaluate whether progabide decreases seizure incidence. Thall and Vail (1990) and Fitzmaurice et al. (2012) analyzed the data by GEE methods.

\subsubsection{Clinical Trial of Antibiotics for Leprosy}

This is a placebo-controlled clinical trial in the Philippines Eversley Child's Sanitarium (Snedecor and Cochran, 1967) affecting 30 patients with leprosy, where study participants were assigned to either one of two antibiotics (treatment A and B) or placebo (drug C), respectively. Until randomization, baseline results were collected for each patient on the number of leprosy bacilli on the body. Once more, the number of leprosy bacilli was reported after several months of treatment. Fitzmaurice et al. (2012) and Cui and Qian (2007) analyzed the data by using GEE techniques, where the question of interest was whether antibiotic treatment (drugs A and B) decreases leprosy bacilli abundance as contrasted with placebo (drug C). 


\subsubsection{Data on Obesity from the Muscatine Coronary Risk Factor Study}

Data on obesity from the research came from the analysis of the Muscatine Cardiovascular Risk Factor (MCRF), a retrospective sample of school-age children in Muscatine, Iowa (Woolson and Clarke, 1984). The review of MCRF aimed to investigate the production and prevalence of risk factors for cardiovascular disease in children. In the MCRF report, measurements on weight and height of five children's cohorts, initially aged 5-7, 7-9, 9-11, 11-13, and 13-15 years, were collected biennially from 1977 through 1981. Data on 4856 boys and girls were gathered. Children have been classified as obese or not obese based on a measure of their weight to age-gender specific standards. Before each assessment, the research procedure required parental consent. This data set was analyzed by Woolson and Clarke (1984), Ekholm and Skinner (1998), Cui and Qian (2007) and Fitzmaurice et al. (2012) among many others. One aim of the MCRF research was to evaluate whether the risk of obesity increased with age and whether boys and girls had the same trend of improvement in obesity. While each child was able to engage in all three surveys, there was a considerable volume of missed obesity data, with fewer than 40 percent of the children presenting accurate results for all three instances of measurement.

Fitzmaurice et al. (2012) reported that the two main reasons for nonresponse are: (1) no parent-signed consent document was issued, and (2) during the day of the test, the child was absent from school. Suppose, the parents of obese children are either more or less inclined to sign the consent form than parents of non-obese children. For instance, parents of children who were obese were also more interested in signing the release form despite concerns about the health effects of obesity; alternatively, they were much less likely to sign the release form due to concerns that the children might be reluctant to engage in the research. In either case, the reason for missing height and weight data has to do with the child's obesity status. Similarly, missingness is linked to the child's obesity status because children who were obese were more prone to be absent on test day attributable, for example, 
to a discomfort overweight. On the other side, if a child was missing on the day of review owing to relocation of jobs by her parents (completely unrelated to the child's health) this absence does not impact the child's obesity status (Fitzmaurice et al., 2012). If the missingness is not linked to the child's obesity status, so this is the case of missing completely at random (MCAR) (where missingness does not rely on the observed and non-observed outcomes), the GEE methodology produce accurate estimates of the regression parameters for longitudinal data analysis. On the other side, if the absence is connected to the obesity level, we have two types of missingness, missing at random (MAR) and not missing at random (NMAR). The ordinary GEE strategies struggle in both instances and may yield inaccurate results.

Robins et al. (1995) suggested a weighted GEE method for the calculation of regression parameters in the case of missing at random (MAR) (where missingness relies on the observed but not the non-observed results). Under MAR missingness, the estimation of weights is probable, but in case of non-ignorable or NMAR (missingness is linked to the particular values which would have been obtained), further advanced methodology is utilized to obtain accurate estimates. 


\section{Chapter 3}

\section{Longitudinal Model for Missing Data}

\subsection{Introduction}

Regression models for binary and count longitudinal responses are studied in this chapter, where the mean response is associated with time and other covariates by a known link function. The main focus is on a monotone missing data pattern, where dropout of a subject is not recuperated by any other measurements for the same subject. The analysis of longitudinal responses accompanied by dropouts is done by incorporating inverse probability weights suggested by Robins et al. (1995) into the GEE approach of Prentice (1988). The empirical properties of weighted GEE estimators are also studied in simulations.

This chapter is organized as follows. Sections 3.2 and 3.3 give a brief review of missing data types and the weighted generalized estimating equation. Section 3.4 provides the model and terminology for incomplete binary longitudinal data and the dropout mechanism. Section 3.4.3 discusses the techniques of generalized estimating equations and weighted generalized estimating equations to evaluate incomplete binary longitudinal data. Section 3.4.4 addresses the asymptotic properties of the WGEE estimators. Section 3.4.5 describes the findings of a simulation study carried out to explore the analytical properties of the weighted GEE method. Section 3.4.6 describes the method by incorporating actual longitudinal data from clinical trials. 
For incomplete count longitudinal data, Section 3.5 discusses the response model and methods of estimation. Section 3.5.3 addresses the empirical properties of the weighted GEE method based on a simulation study. Section 3.6 gives the chapter's conclusions.

\subsection{Missing Data Patterns in Longitudinal Study}

Missingness in data occurs due to many reasons. The problem of missing data commonly arises in health sciences and biological research. A large amount of missingness pattern commonly occurs because a person's response might be measured at one follow-up time and might not be measured at the next follow-up time. This may be due to the fact that some subjects move out of the region so they usually drop out before the study ends, subjects pass away or they see no particular advantage to participate in the longitudinal study or do not like the effect of a treatment. Missing data may occur for many reasons, but when missingness in data occurs, it must be taken into consideration for valid statistical inferences.

The missing data can be fit into three categories, which are based on the link between observed and missing values. It is essential to understand these categories because missing data problems and their solutions are different for each category. Little and Rubin (1987) suggested three different forms of missing data. Let the whole data $\mathbf{Y}$ be symbolized by $\mathbf{Y}=\left(\mathbf{Y}^{o}, \mathbf{Y}^{m}\right)$, where $\mathbf{Y}^{o}$ denotes the observed value and $\mathbf{Y}^{m}$ the missing value of $\mathbf{Y}$ and let $\mathbf{X}$ represent a full set of covariates. Then

$$
\operatorname{pr}(\mathbf{Y} \mid \mathbf{X}, \boldsymbol{\theta})=\operatorname{pr}\left(\mathbf{Y}^{o}, \mathbf{Y}^{m} \mid \mathbf{X}, \boldsymbol{\theta}\right)
$$

is the density function of $\mathbf{Y}$ conditional on some vector of unidentified parameters $\boldsymbol{\theta}$. The response indicator vector is represented by $\mathbf{R}=\left(R_{i t}\right)$, which indicates whether 
each element of $\mathbf{Y}$ is present or absent, so that

$$
R_{i t}= \begin{cases}1 & \text { if } y_{i t} \text { is observed } \\ 0 & \text { if } y_{i t} \text { is missing }\end{cases}
$$

Here $\mathbf{R}$ is a random vector in the model and we assume a joint distribution of $\mathbf{R}$ and $\mathbf{Y}$. The joint distribution can be expressed as

$$
\operatorname{pr}(\mathbf{Y}, \mathbf{R} \mid \mathbf{X}, \boldsymbol{\theta}, \boldsymbol{\tau})=\operatorname{pr}(\mathbf{Y} \mid \mathbf{X}, \boldsymbol{\theta}) \operatorname{pr}(\mathbf{R} \mid \mathbf{Y}, \mathbf{X}, \boldsymbol{\tau})
$$

where $\operatorname{pr}(\mathbf{R} \mid \mathbf{Y}, \mathbf{X}, \boldsymbol{\tau})$ depends on some parameters $\boldsymbol{\tau}$, and is the conditional distribution of $\mathbf{R}$ given $\mathbf{Y}$ for the mechanism of missing data. Three classes or categories of missing data are described below.

1. Missing Completely at Random [MCAR]: MCAR means that the missing data mechanism is not associated with the values of any variables whether it is observed or missing. Under MCAR, missingness is independent of $\mathbf{Y}$, which means that the process of missing data does not rely on missing values as well as observed data values. MCAR occurs when a researcher drops participants by mistake or unintentionally misses questions. MCAR can be represented as

$$
\operatorname{pr}\left(\mathbf{R}=\mathbf{1} \mid \mathbf{Y}^{o}, \mathbf{Y}^{m}, \mathbf{X}, \boldsymbol{\tau}\right)=\operatorname{pr}(\mathbf{R}=\mathbf{1} \mid \mathbf{X}, \boldsymbol{\tau})
$$

2. Missing at Random [MAR]: Under MAR, the occurrence of missing data may be linked to observed values, but not related to missing values of variables. MAR can be represented by

$$
\operatorname{pr}\left(\mathbf{R}=\mathbf{1} \mid \mathbf{Y}^{o}, \mathbf{Y}^{m}, \mathbf{X}, \boldsymbol{\tau}\right)=\operatorname{pr}\left(\mathbf{R}=\mathbf{1} \mid \mathbf{Y}^{o}, \mathbf{X}, \boldsymbol{\tau}\right)
$$

which means that missing values are associated with observed components of $\mathbf{Y}$. When MAR occurs, the probability-based inference does not rely on the missing 
data mechanism (Rubin, 1976).

3. Non-Ignorable [NI] or Not Missing at Random [NMAR]: If the assumption of MAR is violated, then we say that the data are NMAR. Under NMAR, the mechanism of missing data is associated with the missing values, i.e., after adjusting for $\mathbf{X}$ the probability that missing data of $\mathbf{Y}$ depends on $\mathbf{Y}$ itself. It usually happens when individuals do not want to reveal something personal about them. People with higher incomes, for example, may not disclose them on the survey; the missing data mechanism for income cannot be ignored when missingness in income is associated with its value. For NI missing data, complete case analysis can provide biased results because high-income individuals may be missing and only moderate and low-income persons have remained in the sample. In this case, estimates of average income could be less than the actual average. In case of NMAR, we have

$$
\operatorname{pr}(\mathbf{R}=\mathbf{1} \mid \mathbf{Y}, \mathbf{X}, \boldsymbol{\tau})=\operatorname{pr}\left(\mathbf{R}=\mathbf{1} \mid \mathbf{Y}^{o}, \mathbf{Y}^{m}, \mathbf{X}, \boldsymbol{\tau}\right)
$$

Missing data are considered as non-ignorable if missingness depends on a person's unobserved response (Baker and Laird, 1988). In such cases, the missingness mechanism needs to be taken into account. There are many good techniques available for managing ignorable missing data, while non-ignorable missing data requires different approaches and are more challenging.

\subsection{Weighted Generalized Estimating Equations}

The traditional GEE approaches by Prentice (1988) and Liang and Zeger (1986) are useful only when data are missing completely at random (MCAR) (Little and Rubin, 1987). The traditional GEE estimator is biased under the weaker assumption of missing at random (MAR) (Fitzmaurice et al., 2008). Robins et al. (1995) suggested inverse probabilities of first-order weighted GEE method (WGEE) in which the traditional GEE is reweighted by estimated dropout probabilities. The proba- 
bilities creating these weights are attained by modeling missing data indicators as a function of responses. Under the MAR situation, the calculation of these weights is possible. However, in case of non-ignorable missing data, sensitivity analysis must be conducted. The WGEE produces consistent estimators and unbiased estimating equations for mean parameters when the missing data model is MAR and dropout probabilities are specified correctly. There are two types of weighted techniques for estimating regression parameters $\boldsymbol{\beta}$ in case of dropouts: (1) observation-specific weight and (2) subject-specific weight. They both yield reliable estimators when data are MAR.

\subsubsection{Observation-Specific Weighted GEE}

Assume that the response probability weight for $y_{i t}$ is $\nu_{i t}$, which denotes the probability of observing $y_{i t}$, that is, $\nu_{i t}=P\left(R_{i t}=1 \mid \mathbf{X}_{i}, \mathbf{y}_{i}\right)$. Suppose $\boldsymbol{\nu}_{i}$ is a $J \times J$ diagonal matrix whose $t^{\text {th }}$ diagonal element is $\nu_{i t}$. For estimating $\boldsymbol{\beta}$, Robins et al. (1995) proposed the weighted GEE

$$
\mathbf{U}_{\boldsymbol{\beta}}(\boldsymbol{\beta}, \boldsymbol{\alpha})=\sum_{i=1}^{k} \boldsymbol{\nu}_{i}^{-1} \mathbf{D}_{i}^{\prime} \mathbf{V}_{i}^{-1}\left(\mathbf{y}_{i}^{o}-\boldsymbol{\mu}_{i}^{o}(\boldsymbol{\beta})\right)=0
$$

The weights $\nu_{i t}$ are unknown in general, and are calculated with a logistic regression in terms of the MAR assumption. Assume $\lambda_{i t}=P\left(R_{i t}=1 \mid R_{i t-1}=1, \mathbf{X}_{i}, \mathbf{y}_{i}, \boldsymbol{\tau}\right)$ defines the probability of response $y_{i t}$ by its previous observed responses. In terms of the MAR assumption,

$$
\lambda_{i t}=P\left(R_{i t}=1 \mid R_{i t-1}=1, \mathbf{X}_{i}, \mathbf{y}_{i}, \boldsymbol{\tau}\right)=P\left(R_{i t}=1 \mid R_{i t-1}=1, \mathbf{X}_{i}, y_{i 1}, \ldots, y_{i t-1}, \boldsymbol{\tau}\right) .
$$

Here $\lambda_{i t}$ can be predicted from a logistic regression of the form

$$
\operatorname{logit}\left(\lambda_{i t}\right)=\mathbf{C}_{i t}^{\prime} \boldsymbol{\tau},
$$


where predictors $\mathbf{C}_{i t}$ generally contain covariates $\mathbf{x}_{i t}$, earlier responses and visit times indicators. The dropout mechanism indicates that the estimated response probability of $y_{i t}$ can be stated as a product of conditional probabilities

$$
\hat{P}\left(R_{i t}=1 \mid \mathbf{X}_{i}, \mathbf{y}_{i}, \boldsymbol{\tau}\right)=\lambda_{i 1}(\hat{\boldsymbol{\tau}}) \times \cdots \times \lambda_{i t}(\hat{\boldsymbol{\tau}}),
$$

with the estimated weights $\hat{\nu}_{i t}=\hat{P}\left(R_{i t}=1 \mid \mathbf{X}_{i}, \mathbf{y}_{i}, \boldsymbol{\tau}\right)$.

\subsubsection{Subject-Specific Weighted GEE}

In contrast to the observation-specific weighted method, the subject-specific weighted method allocates a particular weight to every subject. In this case, WGEE estimators of $\boldsymbol{\beta}$ and $\boldsymbol{\alpha}$ may be obtained by solving the estimating equations

$$
\begin{aligned}
& \mathbf{U}_{\boldsymbol{\beta}}(\boldsymbol{\beta}, \boldsymbol{\alpha})=\sum_{i=1}^{k} \frac{1}{\nu_{i m}} \mathbf{D}_{i}^{\prime} \mathbf{V}_{i}^{-1}\left(\mathbf{y}_{i}^{o}-\boldsymbol{\mu}_{i}^{o}\right)=0, \\
& \mathbf{U}_{\boldsymbol{\alpha}}(\boldsymbol{\beta}, \boldsymbol{\alpha})=\sum_{i=1}^{k} \frac{1}{\nu_{i m}} \mathbf{L}_{i}^{\prime} \mathbf{W}_{i}^{-1}\left(\mathbf{r}_{i}^{o}-\boldsymbol{\delta}_{i}^{o}\right)=0,
\end{aligned}
$$

where $\nu_{i m}$ is the response probability weight for $i^{\text {th }}$ subject (Fitzmaurice et al., 1995; Preisser et al., 2002). Note that the subject-specific weight $\nu_{i m}$ is defined as a scalar in contrary to the weight matrix $\boldsymbol{\nu}_{i}$. Both weighted methods yield reliable estimators when data are MAR. Here we consider a subject-specific weighted technique, which allocates a particular weight to every subject.

\subsection{Incomplete Binary Longitudinal Model}

\subsubsection{Logistic Response Model}

Assume that $k$ subjects are observed at a fixed set of $T$ time points. Let the $i^{\text {th }}$ subject at visit $t$ provide a binary response variable, denoted by $y_{i t}$, for $1 \leq i \leq k$, and $1 \leq t \leq T$. Let $\mathbf{y}_{i}=\left(y_{i 1}, \ldots, y_{i T}\right)^{\prime}$ denote the $i^{t h}$ binary response vector. Also, 
let the $p \times 1$ covariate vector for the $i^{\text {th }}$ subject at time $t$ be $\mathbf{x}_{i t}=\left(1, x_{i t, 1}, \ldots, x_{i t, p-1}\right)^{\prime}$. The covariates may be deemed fixed or time dependent throughout the observation time. For the $i^{\text {th }}$ individual at time $t$, the marginal probability of success is denoted by $p_{i t}$.

Assume that the marginal distribution of the binary response variable $y_{i t}$ is given by

$$
y_{i t} \sim \operatorname{Bernoulli}\left(p_{i t}\right), \quad i=1, \ldots, k, \quad t=1, \ldots, T
$$

where the probability of success

$$
p_{i t}=E\left(y_{i t} \mid \mathbf{X}_{i}\right)=p\left(y_{i t}=1 \mid \mathbf{X}_{i}\right)
$$

Define vector $\mathbf{p}_{i}=\left(p_{i 1}, \ldots, p_{i T}\right)^{\prime}$. Consider a binary logistic regression model for $p_{i t}$ as

$$
\operatorname{logit}\left(p_{i t}\right)=\log \left(\frac{p_{i t}}{1-p_{i t}}\right)=\mathbf{x}_{i t}^{\prime} \boldsymbol{\beta}
$$

where $\boldsymbol{\beta}=\left(\beta_{0}, \ldots, \beta_{p-1}\right)^{\prime}$ is a $p \times 1$ vector of regression parameters. The marginal variance $v_{i t}$ of $y_{i t}$ is stated as a function of marginal means by

$$
v_{i t}=\operatorname{var}\left(y_{i t} \mid \mathbf{x}_{i t}\right)=p_{i t}\left(1-p_{i t}\right)
$$

Assume that $y_{i t}$ and $y_{i^{\prime}} t^{\prime}$ are uncorrelated when $i \neq i^{\prime}$. Let

$$
\operatorname{corr}\left(y_{i t}, y_{i t^{\prime}}\right)=\alpha_{t t^{\prime}}
$$

denote the correlation between $y_{i t}$ and $y_{i t^{\prime}}$ for given $\mathbf{x}_{i t}$, and let $\boldsymbol{\alpha}=\left(\alpha_{12}, \ldots, \alpha_{1 T}, \alpha_{23}\right.$, $\left.\ldots, \alpha_{T-1, T}\right)^{\prime}$ denote a vector of correlation parameters.

\subsubsection{Dropout Model}

We often encounter attrition in longitudinal studies due to dropouts and delayed enrollments, where participants drop out before the end of the study and do not 
return. For example, members of the panel may drop out in panel surveys because they travel to a place that is inconvenient to the researchers, or, in a clinical study, some participants drop out of the study for known or unknown reasons, possibly due to side effects of drugs, or they have recovered from the disease under investigation. The pattern of attrition is an example of monotonous missing data, where follow-up measurements $y_{i, t+1}, \ldots, y_{i T}$ are missing and all previous measurements $y_{i 1}, \ldots, y_{i, t}$ are observed. The missing data pattern is rarely monotonous in practice, but it is often close to monotonous (Little and Rubin, 1987).

To set up a dropout model, let the indicator variable $R_{i t}$ be 1 if the response $y_{i t}$ is observed and 0 otherwise. Let $\mathbf{R}_{i}=\left(R_{i 1}, R_{i 2}, \ldots, R_{i T}\right)^{\prime}$ denote the response indicators for $\mathbf{y}_{i}=\left(y_{i 1}, \ldots, y_{i T}\right)^{\prime}$. Suppose we have a monotone missingness pattern so that $R_{i 1} \geq \cdots \geq R_{i T}$ and $R_{i 1}=1$ for all subjects. In general, the dropout mechanism depends on the complete vector of responses, which includes the unobserved components of $\mathbf{y}_{i}$ and vector of covariates $\mathbf{X}_{i}$.

Let

$$
\lambda_{i t}=P\left(R_{i t}=1 \mid R_{i 1}=\cdots=R_{i, t-1}=1, \mathbf{y}_{i}, \mathbf{X}_{i}, \boldsymbol{\tau}\right)
$$

be the probability that the subject $i^{t h}$ is observed at time $t$, provided that the subject is observed at previous $t-1$ time points and provided the response vector $\mathbf{y}_{i}$ and covariates $\mathbf{X}_{i}$, where $\boldsymbol{\tau}$ is indicated as the missing data model's "nuisance parameters". Let the observed and non-observed (missing) components of the response vector $\mathbf{y}_{i}$ be denoted by $\mathbf{y}_{i}^{o}$ and $\mathbf{y}_{i}^{m}$. For MCAR (missing completely at random), $\lambda_{i t}=P\left(R_{i t}=1 \mid R_{i 1}, \ldots, R_{i, t-1}=1, \mathbf{X}_{i}, \boldsymbol{\tau}\right)$ and for MAR (missing at random) $\lambda_{i t}=P\left(R_{i t}=1 \mid R_{i 1}, \ldots, R_{i, t-1}=1, \mathbf{y}_{i}^{o}, \mathbf{X}_{i}, \boldsymbol{\tau}\right)$. For NI (non-ignorable) missingness, $\lambda_{i t}$ depends on the non-observed response variables.

In the case of dropouts, the binary indicator random vector $\mathbf{R}_{i}=\left(R_{i 1}, \ldots, R_{i T}\right)^{\prime}$ be defined by a single random variable as

$$
M_{i}=1+\sum_{t=1}^{T} R_{i t}
$$


which indicates the dropout time. The dropout or missing data process can be defined by

$$
\nu_{i m_{i}}=f_{M_{i}}\left(m_{i} \mid \mathbf{y}_{i}, \mathbf{X}_{i}, \boldsymbol{\tau}\right)=P\left(M_{i}=m_{i} \mid \mathbf{y}_{i}, \mathbf{X}_{i}, \boldsymbol{\tau}\right)
$$

The value of $M_{i}$ lies between 2 and $T+1$ if all subjects are observed at the first visit, where the maximum value of $T+1$ corresponds to a full sequence of measurements. The probability that the $i^{\text {th }}$ subject drops out at the $m^{\text {th }}$ visit is given by

$$
\begin{aligned}
P\left(M_{i}=m \mid \mathbf{y}_{i}, \mathbf{X}_{i}, \boldsymbol{\tau}\right) & =P\left(R_{i 2}=\cdots=R_{i, m-1}=1, R_{i m}=0 \mid y_{i 1}, \ldots, y_{i m}, \mathbf{X}_{i}, \boldsymbol{\tau}\right) \\
& =\left\{\prod_{t=2}^{m-1} P\left(R_{i t}=1 \mid R_{i 1}=\cdots=R_{i, t-1}=1, y_{i 1}, \ldots, y_{i t}, \mathbf{X}_{i}, \boldsymbol{\tau}\right)\right\} \\
& \times P\left(R_{i m}=0 \mid R_{i 1}=\cdots=R_{i m-1}=1, y_{i 1}, \ldots, y_{i m}, \mathbf{X}_{i}, \boldsymbol{\tau}\right)^{I\{m \leq T\}},
\end{aligned}
$$

where $I\{\}$ is an indicator variable.

\subsubsection{Methods of Estimation}

\subsubsection{Generalized Estimating Equations}

This section addresses the estimation of model parameters using the weighted generalized estimating equation (WGEE) and generalized estimating equation (GEE). Here the estimation of association parameters $\boldsymbol{\alpha}$ and regression parameters $\boldsymbol{\beta}$ is of main interest, and $\boldsymbol{\tau}$ is considered as the nuisance parameter of the missing data model. Using the traditional method, the longitudinal data may be analyzed by ignoring the missing observations. In such a case, for the joint estimation of the re-

gression parameters $\boldsymbol{\beta}$ and association parameters $\boldsymbol{\alpha}$, the GEE approach of Prentice (1988) solves the estimating equations

$$
\begin{aligned}
& \mathbf{U}_{\boldsymbol{\beta}}(\boldsymbol{\beta}, \boldsymbol{\alpha})=\sum_{i=1}^{k} \mathbf{D}_{i}^{\prime} \mathbf{V}_{i}^{-1}\left(\mathbf{y}_{i}^{o}-\mathbf{p}_{i}^{o}(\boldsymbol{\beta})\right)=0, \\
& \mathbf{U}_{\boldsymbol{\alpha}}(\boldsymbol{\beta}, \boldsymbol{\alpha})=\sum_{i=1}^{k} \mathbf{L}_{i}^{\prime} \mathbf{W}_{i}^{-1}\left(\mathbf{r}_{i}^{o}-\boldsymbol{\delta}_{i}^{o}(\boldsymbol{\alpha})\right)=0,
\end{aligned}
$$


where $\mathbf{D}_{i}=\partial \mathbf{p}_{i}^{o} / \partial \boldsymbol{\beta}=\left(\partial \mathbf{p}_{i}^{o} / \partial \boldsymbol{\eta}_{i}\right)\left(\partial \boldsymbol{\eta}_{i} / \partial \boldsymbol{\beta}\right)=\boldsymbol{\Delta}_{i} \mathbf{X}_{i}, \mathbf{V}_{i}=\mathbf{A}_{i}^{o(1 / 2)} \boldsymbol{\rho}^{o}(\boldsymbol{\alpha}) \mathbf{A}_{i}^{o(1 / 2)}$, and $\boldsymbol{\rho}^{o}(\boldsymbol{\alpha})$ is a working correlation matrix for $\mathbf{y}_{i}^{o}$, depending on the vector of correlation parameters $\boldsymbol{\alpha}$. For the correlation parameters $\boldsymbol{\alpha}$, the GEE estimator can be attained by solving estimating equation (3.17), where $\mathbf{L}_{i}=\partial \boldsymbol{\delta}_{i}^{o} / \partial \boldsymbol{\alpha}, \mathbf{W}_{i}=\operatorname{diag}\left\{\operatorname{var}\left(\mathbf{r}_{i}^{o}\right)\right\}$, $\mathbf{r}_{i}^{o}$ is observed component of $\mathbf{r}_{i}=\left(r_{i 12}, \ldots, r_{i 1 T}, r_{i 23}, \ldots, r_{i, T-1, T}\right)^{\prime}$, with $r_{i t u}$ being the sample correlation

$$
r_{i t u}=r_{i t u}(\boldsymbol{\beta})=\frac{\left(y_{i t}-p_{i t}\right)\left(y_{i u}-p_{i u}\right)}{\left(p_{i t} q_{i t} p_{i u} q_{i u}\right)^{1 / 2}}
$$

and $\boldsymbol{\delta}_{i}^{o}=E\left(\mathbf{r}_{i}^{o}\right)$. We can show that

$$
\operatorname{var}\left(r_{i t u}\right)=\omega_{i t u}=1+\left(1-2 p_{i t}\right)\left(1-2 p_{i u}\right)\left(p_{i t} q_{i t} p_{i u} q_{i u}\right)^{-1 / 2} \delta_{i t u}-\delta_{i t u}^{2},
$$

for $t<u<T$.

Note that the ordinary GEE provides biased estimators when the missingness is MAR or non-ignorable (Fitzmaurice et al., 1995). Next, the estimates of $\boldsymbol{\beta}$ and $\boldsymbol{\alpha}$ are obtained by using the weighted GEE method introduced by Robins et al. (1995) in the case of dropouts.

\subsubsection{Weighted Generalized Estimating Equations}

When missing data are MAR, Robins et al. (1995) suggested the weighted GEE technique in which the GEE is reweighted by estimated dropout probabilities. In particular, the WGEE obtains $\hat{\boldsymbol{\beta}}$ by solving

$$
\hat{\mathbf{U}}_{\boldsymbol{\beta}}(\boldsymbol{\beta}, \boldsymbol{\alpha})=\sum_{i=1}^{k} \frac{1}{\nu_{i m}} \mathbf{D}_{i}^{\prime} \mathbf{V}_{i}^{-1}\left(\mathbf{y}_{i}^{o}-\mathbf{p}_{i}^{o}(\boldsymbol{\beta})\right)=0
$$

where $\nu_{i m}$ provides response probability weights as defined in (3.15). The estimating equation (3.20) is unbiased at true $\boldsymbol{\beta}$ if $\nu_{i m}$ is specified correctly (Fitzmaurice et al., 1995). Similarly, to estimate the association parameters $\boldsymbol{\alpha}$, following Prentice (1988) 
we consider solving the weighted estimating equations

$$
\begin{aligned}
\hat{\mathbf{U}}_{\boldsymbol{\alpha}}(\boldsymbol{\beta}, \boldsymbol{\alpha}) & =\sum_{i=1}^{k} \frac{1}{\nu_{i m}} \mathbf{L}_{i}^{\prime} \mathbf{W}_{i}^{-1}\left(\mathbf{r}_{i}^{o}-\boldsymbol{\delta}_{i}^{o}(\boldsymbol{\alpha})\right) \\
& =\sum_{i=1}^{k} \sum_{m_{i}=2}^{T+1} \frac{I\left(M_{i}=m_{i}\right)}{\nu_{i m_{i}}} \mathbf{L}_{i}^{\prime}\left(m_{i}\right) \mathbf{W}_{i}^{-1}\left(m_{i}\right)\left\{\mathbf{r}_{i}\left(m_{i}\right)-\boldsymbol{\delta}_{i}\left(m_{i}\right)\right\}=0,
\end{aligned}
$$

where $\mathbf{L}_{i}=\partial \boldsymbol{\delta}_{i}^{o} / \partial \boldsymbol{\alpha}$ and $\mathbf{W}_{i}=\operatorname{diag}\left\{\operatorname{var}\left(\mathbf{r}_{i}^{o}\right)\right\} ; \mathbf{r}_{i}\left(m_{i}\right)$ and $\boldsymbol{\delta}_{i}\left(m_{i}\right)$ correspond to the $m_{i}-1$ observed values of the response variable. For instance, if $m_{i}=m$, then $\mathbf{r}_{i}(m)=\left(r_{i 12}, \ldots, r_{i 1, m-1}, r_{i 23}, \ldots, r_{i, m-2, m-1}\right)^{\prime}$. Since

$$
\nu_{i m_{i}}=P\left(M_{i}=m_{i} \mid \mathbf{y}_{i}, \mathbf{X}_{i}, \boldsymbol{\tau}\right)=E_{M_{i}} \mid \mathbf{y}_{i}\left[I\left\{M_{i}=m_{i}\right\}\right]
$$

we have

$$
\begin{aligned}
& E\left[\frac{I\left\{M_{i}=m_{i}\right\}}{\nu_{i m_{i}}} \mathbf{L}_{i}^{\prime}\left(m_{i}\right) \mathbf{W}_{i}^{-1}\left(m_{i}\right)\left\{\mathbf{r}_{i}\left(m_{i}\right)-\boldsymbol{\delta}_{i}\left(m_{i}\right)\right\}\right] \\
& =E_{\mathbf{y}_{i}}\left[\mathbf{L}_{i}^{\prime}\left(m_{i}\right) \mathbf{W}_{i}^{-1}\left(m_{i}\right)\left\{\mathbf{r}_{i}\left(m_{i}\right)-\boldsymbol{\delta}_{i}\left(m_{i}\right)\right\} E_{M_{i} \mid \mathbf{y}_{i}}\left\{\frac{I\left\{M_{i}=m_{i}\right\}}{\nu_{i m_{i}}}\right\}\right] \\
& =E_{\mathbf{y}_{i}}\left[\mathbf{L}_{i}^{\prime}\left(m_{i}\right) \mathbf{W}_{i}^{-1}\left(m_{i}\right)\left\{\mathbf{r}_{i}\left(m_{i}\right)-\boldsymbol{\delta}_{i}\left(m_{i}\right)\right\}\right]=0 .
\end{aligned}
$$

Thus if $\nu_{i m_{i}}$ is specified correctly then estimating equation (3.21) is unbiased. Since the estimating equations for $\boldsymbol{\beta}$ and $\boldsymbol{\alpha}$ are both unbiased for 0, WGEE estimates are consistent for $(\boldsymbol{\beta}, \boldsymbol{\alpha})$, as evident from the theory of standard moments method of estimation. If the dropout probabilities $\nu_{i m_{i}}$ are consistently calculated, then the WGEE estimators would still provide reliable estimators of $(\boldsymbol{\beta}, \boldsymbol{\alpha})$ (Robins et al., 1995).

To find the estimates of $(\boldsymbol{\beta}, \boldsymbol{\alpha})$, the iterative procedure begins with some initial values $\left(\boldsymbol{\beta}_{o}, \boldsymbol{\alpha}_{o}\right)$ and produces updated values $\left(\boldsymbol{\beta}_{s+1}, \boldsymbol{\alpha}_{s+1}\right)$ from interim values $\left(\boldsymbol{\beta}_{s}, \boldsymbol{\alpha}_{s}\right)$ by means of the iterative equations

$$
\boldsymbol{\beta}_{s+1}=\boldsymbol{\beta}_{s}+\left(\sum_{i=1}^{k} \frac{1}{\nu_{i m}} \mathbf{D}_{i}^{\prime} \mathbf{V}_{i}^{-1} \mathbf{D}_{i}\right)^{-1} \sum_{i=1}^{k} \frac{1}{\nu_{i m}} \mathbf{D}_{i}^{\prime} \mathbf{V}_{i}^{-1}\left(\mathbf{y}_{i}^{o}-\mathbf{p}_{i}^{o}(\boldsymbol{\beta})\right)
$$




$$
\boldsymbol{\alpha}_{s+1}=\boldsymbol{\alpha}_{s}+\left(\sum_{i=1}^{k} \frac{1}{\nu_{i m}} \mathbf{L}_{i}^{\prime} \mathbf{W}_{i}^{-1} \mathbf{L}_{i}\right)^{-1} \sum_{i=1}^{k} \frac{1}{\nu_{i m}} \mathbf{L}_{i}^{\prime} \mathbf{W}_{i}^{-1}\left(\mathbf{r}_{i}^{o}-\boldsymbol{\delta}_{i}^{o}(\boldsymbol{\alpha})\right),
$$

for $s=0,1,2, \ldots$, where for every estimating equation, the second term on the right side is evaluated at the current estimates $\left(\boldsymbol{\beta}_{s}, \boldsymbol{\alpha}_{s}\right)$.

\subsubsection{Approximate Variance of WGEE Estimators}

Following White (1982), the variance-covariance matrix of WGEE estimators $\hat{\boldsymbol{\beta}}$ and $\hat{\boldsymbol{\alpha}}$ can be approximated by using sandwich type estimators. In particular, variance of $\hat{\boldsymbol{\beta}}$ is approximated by

$$
\mathbf{V}(\hat{\boldsymbol{\beta}})=\mathbf{H}_{\boldsymbol{\beta}}^{-1} \mathbf{O}_{\boldsymbol{\beta}} \mathbf{H}_{\boldsymbol{\beta}}^{-1}
$$

where $\mathbf{H}_{\boldsymbol{\beta}}=\sum_{i=1}^{k} \frac{1}{\nu_{i m}} \mathbf{D}_{i}^{\prime} \mathbf{V}_{i}^{-1} \mathbf{D}_{i}$ with

$\mathbf{O}_{\boldsymbol{\beta}}=\sum_{i=1}^{k} \mathbf{S}_{\boldsymbol{\beta}, i} \mathbf{S}_{\boldsymbol{\beta}, i}^{\prime}$, with $\mathbf{S}_{\boldsymbol{\beta}, i}=\frac{1}{\nu_{i m}} \mathbf{D}_{i}^{\prime} \mathbf{V}_{i}^{-1}\left(\mathbf{y}_{i}^{o}-\mathbf{p}_{i}^{o}\right)$. Similarly, variance of $\hat{\boldsymbol{\alpha}}$ may be approximated by

$$
\mathrm{V}(\hat{\alpha})=\mathbf{H}_{\alpha}^{-1} \mathbf{O}_{\alpha} \mathbf{H}_{\alpha}^{-1}
$$

where $\mathbf{H}_{\boldsymbol{\alpha}}=\sum_{i=1}^{k} \frac{1}{\nu_{i m}} \mathbf{L}_{i}^{\prime} \mathbf{W}_{i}^{-1} \mathbf{L}_{i}$ and $\mathbf{O}_{\boldsymbol{\alpha}}=\sum_{i=1}^{k} \mathbf{S}_{\boldsymbol{\alpha}, i} \mathbf{S}_{\boldsymbol{\alpha}, i}^{\prime}$, with $\mathbf{S}_{\boldsymbol{\alpha}, i}=\frac{1}{\nu_{i m}} \mathbf{L}_{i}^{\prime} \mathbf{W}_{i}^{-1}\left(\mathbf{r}_{i}^{o}-\boldsymbol{\delta}_{i}^{o}\right)$. The matrices $\mathbf{H}_{\boldsymbol{\alpha}}, \mathbf{H}_{\boldsymbol{\beta}}$ and $\mathbf{O}_{\boldsymbol{\alpha}}, \mathbf{O}_{\boldsymbol{\beta}}$ are evaluated the WGEE estimators $\hat{\boldsymbol{\alpha}}$ and $\hat{\boldsymbol{\beta}}$.

\subsubsection{Asymptotic Properties of WGEE Estimators}

Equations (3.20) and (3.21) can be written jointly for $\boldsymbol{\beta}, \boldsymbol{\alpha}$ as

$$
\sum_{i=1}^{k} \boldsymbol{\psi}_{i}(\hat{\boldsymbol{\beta}}, \hat{\boldsymbol{\alpha}})=\mathbf{0}
$$

Using first-order Taylor series approximation about true values $(\boldsymbol{\beta}, \boldsymbol{\alpha})$, we can write

$$
0 \equiv \sum_{i=1}^{k} \boldsymbol{\psi}_{i}(\hat{\boldsymbol{\beta}}, \hat{\boldsymbol{\alpha}}) \cong \sum_{i=1}^{k} \boldsymbol{\psi}_{i}(\boldsymbol{\beta}, \boldsymbol{\alpha})+\left[\sum_{i=1}^{k} \frac{\partial \boldsymbol{\psi}_{i}(\boldsymbol{\beta}, \boldsymbol{\alpha})}{\boldsymbol{\partial}(\boldsymbol{\beta}, \boldsymbol{\alpha})}\right]^{\prime}\left[\left(\begin{array}{l}
\hat{\boldsymbol{\beta}} \\
\hat{\boldsymbol{\alpha}}
\end{array}\right)-\left(\begin{array}{l}
\boldsymbol{\beta} \\
\boldsymbol{\alpha}
\end{array}\right)\right]
$$




$$
\begin{gathered}
\Rightarrow\left[\left(\begin{array}{l}
\hat{\boldsymbol{\beta}} \\
\hat{\boldsymbol{\alpha}}
\end{array}\right)-\left(\begin{array}{l}
\boldsymbol{\beta} \\
\boldsymbol{\alpha}
\end{array}\right)\right] \cong\left\{-\left[\sum_{i=1}^{k} \frac{\boldsymbol{\partial} \boldsymbol{\psi}_{i}(\boldsymbol{\beta}, \boldsymbol{\alpha})}{\boldsymbol{\partial}(\boldsymbol{\beta}, \boldsymbol{\alpha})}\right]^{\prime}\right\}^{-1} \sum_{i=1}^{k} \boldsymbol{\psi}_{i}(\boldsymbol{\beta}, \boldsymbol{\alpha}) \\
\Rightarrow k^{1 / 2}\left[\left(\begin{array}{l}
\hat{\boldsymbol{\beta}} \\
\hat{\boldsymbol{\alpha}}
\end{array}\right)-\left(\begin{array}{l}
\boldsymbol{\beta} \\
\boldsymbol{\alpha}
\end{array}\right)\right] \cong\left\{-k^{-1}\left[\sum_{i=1}^{k} \frac{\boldsymbol{\partial} \boldsymbol{\psi}_{i}(\boldsymbol{\beta}, \boldsymbol{\alpha})}{\boldsymbol{\partial}(\boldsymbol{\beta}, \boldsymbol{\alpha})}\right]^{\prime}\right\}^{-1}\left[k^{-1 / 2} \sum_{i=1}^{k} \boldsymbol{\psi}_{i}(\boldsymbol{\beta}, \boldsymbol{\alpha})\right]
\end{gathered}
$$

Thus $k^{1 / 2}\left[(\hat{\boldsymbol{\beta}}-\boldsymbol{\beta})^{\prime},(\hat{\boldsymbol{\alpha}}-\boldsymbol{\alpha})^{\prime}\right]^{\prime}$ can be approximated under some regularity conditions by

$$
\left[\begin{array}{ll}
-k^{-1} \frac{\partial \hat{\boldsymbol{U}}_{\boldsymbol{\beta}}(\boldsymbol{\beta}, \boldsymbol{\alpha})}{\partial \hat{\boldsymbol{\beta}}} & -k^{-1} \frac{\partial \hat{\boldsymbol{U}}_{\boldsymbol{\beta}}(\boldsymbol{\beta}, \boldsymbol{\alpha})}{\partial \hat{\alpha}} \\
-k^{-1} \frac{\partial \hat{\boldsymbol{U}}_{\alpha}(\boldsymbol{\beta}, \boldsymbol{\alpha})}{\partial \hat{\boldsymbol{\beta}}} & -k^{-1} \frac{\partial \hat{\boldsymbol{U}}_{\boldsymbol{\alpha}}(\boldsymbol{\beta}, \boldsymbol{\alpha})}{\partial \hat{\boldsymbol{\alpha}}}
\end{array}\right]^{-1}\left[\begin{array}{l}
k^{-1 / 2} \hat{\mathbf{U}}_{\boldsymbol{\beta}}(\boldsymbol{\beta}, \boldsymbol{\alpha}) \\
k^{-1 / 2} \hat{\mathbf{U}}_{\boldsymbol{\alpha}}(\boldsymbol{\beta}, \boldsymbol{\alpha})
\end{array}\right] \cong \mathrm{Q}^{-1} \mathrm{G} .
$$

Hence the variance can be written as

$$
\operatorname{var}\left(\mathbf{Q}^{-1} \mathbf{G}\right)=\left(\mathbf{Q}^{-1}\right) \operatorname{var}(\mathbf{G})\left(\mathbf{Q}^{-1}\right)^{\prime}
$$

where $\operatorname{var}(\mathbf{G})$ can be obtained as

$$
\begin{gathered}
\operatorname{var}(\mathbf{G})=k^{-1}\left[\begin{array}{cc}
\operatorname{var}\left(\hat{\mathbf{U}}_{\boldsymbol{\beta}}\right) & \operatorname{cov}\left(\hat{\mathbf{U}}_{\boldsymbol{\beta}}, \hat{\mathbf{U}}_{\boldsymbol{\alpha}}\right) \\
\operatorname{cov}\left(\hat{\mathbf{U}}_{\boldsymbol{\beta}}, \hat{\mathbf{U}}_{\boldsymbol{\alpha}}\right) & \operatorname{var}\left(\hat{\mathbf{U}}_{\boldsymbol{\alpha}}\right)
\end{array}\right] \\
=k^{-1}\left[\begin{array}{cc}
\sum_{i=1}^{k} \frac{1}{\nu_{i m}^{2}} \mathbf{D}_{i}^{\prime} \mathbf{V}_{i}^{-1} \operatorname{var}\left(\mathbf{y}_{i}^{o}\right) \mathbf{V}_{i}^{-1} \mathbf{D}_{i} & \sum_{i=1}^{k} \frac{1}{\nu_{i m}^{2}} \mathbf{D}_{i}^{\prime} \mathbf{V}_{i}^{-1} \operatorname{cov}\left(\mathbf{y}_{i}^{o}, \mathbf{r}_{i}^{o}\right) \mathbf{W}_{i}^{-1} \mathbf{L}_{i} \\
\sum_{i=1}^{k} \frac{1}{\nu_{i m}^{2}} \mathbf{D}_{i}^{\prime} \mathbf{V}_{i}^{-1} \operatorname{cov}\left(\mathbf{y}_{i}^{o}, \mathbf{r}_{i}^{o}\right) \mathbf{V}_{i}^{-1} \mathbf{D}_{i} & \sum_{i=1}^{k} \frac{1}{\nu_{i m}^{2}} \mathbf{L}_{i}^{\prime} \mathbf{W}_{i}^{-1} \operatorname{var}\left(\mathbf{r}_{i}^{o}\right) \mathbf{W}_{i}^{-1} \mathbf{L}_{i}
\end{array}\right] \\
\cong k^{-1}\left(\begin{array}{cc}
\boldsymbol{\Gamma}_{\mathbf{1 1}} & \boldsymbol{\Gamma}_{\mathbf{1 2}} \\
\boldsymbol{\Gamma}_{\mathbf{2 1}} & \boldsymbol{\Gamma}_{\mathbf{2 2}}
\end{array}\right)
\end{gathered}
$$

with approximations

$$
\begin{gathered}
\operatorname{var}\left(\mathbf{y}_{i}^{o}\right) \cong\left(\mathbf{y}_{i}^{o}-\mathbf{p}_{i}^{o}\right)\left(\mathbf{y}_{i}^{o}-\mathbf{p}_{i}^{o}\right)^{\prime} \\
\operatorname{cov}\left(\mathbf{y}_{i}^{o}, \mathbf{r}_{i}^{o}\right) \cong\left(\mathbf{y}_{i}^{o}-\mathbf{p}_{i}^{o}\right)\left(\mathbf{r}_{i}^{o}-\boldsymbol{\delta}_{i}^{o}\right)^{\prime} \\
\operatorname{var}\left(\mathbf{r}_{i}^{o}\right) \cong\left(\mathbf{r}_{i}^{o}-\boldsymbol{\delta}_{i}^{o}\right)\left(\mathbf{r}_{i}^{o}-\boldsymbol{\delta}_{i}^{o}\right)^{\prime}
\end{gathered}
$$

We have already seen that the estimating equations (3.20) and (3.21) for $\boldsymbol{\beta}$ and $\boldsymbol{\alpha}$ are unbiased. Hence the score function $\mathbf{G}$ has an asymptotic normal distribution 
with mean zero and variance

$$
\Sigma_{\mathbf{G}}=k^{-1}\left(\begin{array}{ll}
\Gamma_{11} & \Gamma_{12} \\
\Gamma_{21} & \Gamma_{22}
\end{array}\right)
$$

Thus $\left.k^{1 / 2}\left[(\hat{\boldsymbol{\beta}}-\boldsymbol{\beta})^{\prime},(\hat{\boldsymbol{\alpha}}-\boldsymbol{\alpha})^{\prime}\right)\right]^{\prime}=\mathbf{Q}^{-\mathbf{1}} \mathbf{G}$ has an asymptotic multivariate normal distribution with mean 0 and variance

$$
\boldsymbol{\Sigma}=\lim _{k \rightarrow \infty}\left(\mathbf{Q}^{-\mathbf{1}}\right) \boldsymbol{\Sigma}_{\mathbf{G}} \lim _{k \rightarrow \infty}\left(\mathbf{Q}^{-\mathbf{1}}\right)^{\prime}
$$

To calculate the variance in (3.35), we can show

$$
\begin{gathered}
-k^{-1} \frac{\partial \hat{\mathbf{U}}_{\boldsymbol{\beta}}(\boldsymbol{\beta}, \boldsymbol{\alpha})}{\boldsymbol{\partial} \boldsymbol{\beta}}=-k^{-1} \sum_{i=1}^{k} \frac{1}{\nu_{i m}} \mathbf{D}_{i}^{\prime} \mathbf{V}_{i}^{-1} \frac{\boldsymbol{\partial}\left(\mathbf{y}_{i}^{o}-\mathbf{p}_{i}^{o}\right)}{\boldsymbol{\partial} \boldsymbol{\beta}} \\
-k^{-1} \sum_{i=1}^{k} \frac{1}{\nu_{i m}} \frac{\partial \mathbf{D}_{i}^{\prime} \mathbf{V}_{i}^{-1}}{\boldsymbol{\partial} \boldsymbol{\beta}}\left(\mathbf{y}_{i}^{o}-\mathbf{p}_{i}^{o}\right) \\
=k^{-1} \sum_{i=1}^{k} \frac{1}{\nu_{i m}} \mathbf{D}_{i}^{\prime} \mathbf{V}_{i}^{-1} \mathbf{D}_{i}+o_{p}(1) .
\end{gathered}
$$

Similarly, we can show

$$
\begin{aligned}
& -k^{-1} \frac{\partial \hat{\mathbf{U}}_{\boldsymbol{\beta}}(\boldsymbol{\beta}, \boldsymbol{\alpha})}{\boldsymbol{\partial \alpha}}=-k^{-1} \sum_{i=1}^{k} \frac{1}{\nu_{i m}} \mathbf{D}_{i}^{\prime} \frac{\partial \mathbf{V}_{i}^{-1}}{\partial \boldsymbol{\alpha}}\left(\mathbf{y}_{i}^{o}-\mathbf{p}_{i}^{o}\right)=o_{p}(1) \\
& -k^{-1} \frac{\partial \hat{\mathbf{U}}_{\boldsymbol{\alpha}}(\boldsymbol{\beta}, \boldsymbol{\alpha})}{\boldsymbol{\partial} \boldsymbol{\alpha}}=-k^{-1} \sum_{i=1}^{k} \frac{1}{\nu_{i m}} \mathbf{L}_{i}^{\prime} \mathbf{W}_{i}^{-1} \frac{\boldsymbol{\partial}\left(\mathbf{r}_{i}^{o}-\boldsymbol{\delta}_{i}^{o}\right)}{\boldsymbol{\partial} \boldsymbol{\alpha}} \\
& -k^{-1} \sum_{i=1}^{k} \frac{1}{\nu_{i m}} \frac{\partial \mathbf{L}_{i}^{\prime} \mathbf{W}_{i}^{-1}}{\partial \boldsymbol{\alpha}}\left(\mathbf{r}_{i}^{o}-\boldsymbol{\delta}_{i}^{o}\right) \\
& =k^{-1} \sum_{i=1}^{k} \frac{1}{\nu_{i m}} \mathbf{L}_{i}^{\prime} \mathbf{W}_{i}^{-1} \mathbf{L}_{i}+o_{p}(1),
\end{aligned}
$$

and

$$
-k^{-1} \frac{\partial \hat{\mathbf{U}}_{\boldsymbol{\alpha}}(\boldsymbol{\beta}, \boldsymbol{\alpha})}{\boldsymbol{\partial} \boldsymbol{\beta}}=-k^{-1} \sum_{i=1}^{k} \frac{1}{\nu_{i m}} \mathbf{L}_{i}^{\prime} \mathbf{W}_{i}^{-1} \frac{\boldsymbol{\partial}\left(\mathbf{r}_{i}^{o}-\boldsymbol{\delta}_{i}^{o}\right)}{\boldsymbol{\partial} \boldsymbol{\beta}}
$$




$$
\begin{aligned}
& -k^{-1} \sum_{i=1}^{k} \frac{1}{\nu_{i m}} \frac{\partial \mathbf{L}_{i}^{\prime} \mathbf{W}_{i}^{-1}}{\boldsymbol{\partial} \boldsymbol{\beta}}\left(\mathbf{r}_{i}^{o}-\boldsymbol{\delta}_{i}^{o}\right) \\
& =k^{-1} \sum_{i=1}^{k} \frac{1}{\nu_{i m}} \mathbf{L}_{i}^{\prime} \mathbf{W}_{i}^{-1} \frac{\partial \mathbf{r}_{i}^{o}}{\boldsymbol{\partial} \boldsymbol{\beta}}+o_{p}(1)
\end{aligned}
$$

Here we can show

$$
\begin{gathered}
\frac{\partial r_{i t u}}{\partial \beta_{j}}=-\left\{\frac{\partial p_{i t}}{\partial \beta_{j}}\left(y_{i u}-p_{i u}\right)+\frac{\partial p_{i u}}{\partial \beta_{j}}\left(y_{i t}-p_{i t}\right)+\frac{1}{2}\left(y_{i t}-p_{i t}\right)\left(y_{i u}-p_{i u}\right)\right. \\
\left.\times\left[\left(1-2 p_{i t}\right) p_{i t}^{-1} q_{i t}^{-1} \frac{\partial p_{i t}}{\partial \beta_{j}}+\left(1-2 p_{i u}\right) p_{i u}^{-1} q_{i u}^{-1} \frac{\partial p_{i u}}{\partial \beta_{j}}\right]\right\}\left(p_{i t} q_{i t} p_{i u} q_{i u}\right)^{-1 / 2} .
\end{gathered}
$$

Hence as $k \rightarrow \infty$, we can write

$$
\mathbf{Q}=k^{-1}\left[\begin{array}{cc}
\sum_{i=1}^{k} \frac{1}{\nu_{i m}} \mathbf{D}_{i}^{\prime} \mathbf{V}_{i}^{-1} \mathbf{D}_{i} & 0 \\
\sum_{i=1}^{k} \frac{1}{\nu_{i m}} \mathbf{L}_{i}^{\prime} \mathbf{W}_{i}^{-1} \frac{\partial \mathbf{r}_{i}^{o}}{\boldsymbol{\partial} \boldsymbol{\beta}} & \sum_{i=1}^{k} \frac{1}{\nu_{i m}} \mathbf{L}_{i}^{\prime} \mathbf{W}_{i}^{-1} \mathbf{L}_{i}
\end{array}\right]
$$

and

$$
\mathbf{Q}^{-\mathbf{1}}=k\left[\begin{array}{cc}
\left(\sum_{i=1}^{k} \frac{1}{\nu_{i m}} \mathbf{D}_{i}^{\prime} \mathbf{V}_{i}^{-1} \mathbf{D}_{i}\right)^{-1} & \mathbf{0} \\
\mathbf{B}^{*} & \left(\sum_{i=1}^{k} \frac{1}{\nu_{i m}} \mathbf{L}_{i}^{\prime} \mathbf{W}_{i}^{-1} \mathbf{L}_{i}\right)^{-1}
\end{array}\right]
$$

where

$$
\mathbf{B}^{*}=\left(\sum_{i=1}^{k} \frac{1}{\nu_{i m}} \mathbf{L}_{i}^{\prime} \mathbf{W}_{i}^{-1} \mathbf{L}_{i}\right)^{-1}\left(\sum_{i=1}^{k} \frac{1}{\nu_{i m}} \mathbf{L}_{i}^{\prime} \mathbf{W}_{i}^{-1} \frac{\partial \mathbf{r}_{i}^{o}}{\boldsymbol{\partial} \boldsymbol{\beta}}\right)\left(\sum_{i=1}^{k} \frac{1}{\nu_{i m}} \mathbf{D}_{i}^{\prime} \mathbf{V}_{i}^{-1} \mathbf{D}_{i}\right)^{-1}
$$

Then the asymptotic variance matrix of $\left.k^{1 / 2}\left[(\hat{\boldsymbol{\beta}}-\boldsymbol{\beta})^{\prime},(\hat{\boldsymbol{\alpha}}-\boldsymbol{\alpha})^{\prime}\right)\right]^{\prime}$ can be stated as

$$
\Sigma=\mathrm{Q}^{-1} \Sigma_{\mathrm{G}}\left(\mathbf{Q}^{-1}\right)^{\prime}
$$

where $\boldsymbol{\Sigma}_{\mathbf{G}}$ and $\mathbf{Q}$ are defined in (3.34) and (3.41), respectively.

\subsubsection{Simulation Study}

Two sets of simulations were carried out under incomplete longitudinal data to test the empirical properties of the GEE and WGEE estimators. Under correctly specified MAR models, estimators were studied in the first set, while under mis- 
specified non-ignorable missing data models, estimator's robustness properties were studied in the second set. In both simulations sets, data were produced under exchangeable and serial correlation structures among longitudinal responses.

Three techniques were compared in the simulations:

1. GEE : Both $\boldsymbol{\alpha}$ and $\boldsymbol{\beta}$ are estimated by solving the unweighted GEEs (3.16) and (3.17) following Prentice (1988).

2. WGEE1: $\boldsymbol{\beta}$ is estimated by solving the weighted GEEs (3.20), but $\boldsymbol{\alpha}$ is estimated by solving the unweighted GEEs (3.17).

3. WGEE2: Both $\boldsymbol{\alpha}$ and $\boldsymbol{\beta}$ are estimated by solving weighted GEEs (3.20) and $(3.21)$.

\subsubsection{Response Models}

In the first set of simulations, data were generated under the MAR model, where binary responses where obtained at three follow-up times using the marginal mean response model

$$
\operatorname{logit}\left[E\left(y_{i t} \mid \mathbf{x}_{i t}, \boldsymbol{\beta}, \alpha\right)\right]=\beta_{0}+\beta_{1} x_{i}+\beta_{2} t, \quad t=1,2,3 .
$$

Here the binary covariate $x_{i}$ was chosen as a group indicator with $P\left(x_{i}=1\right)=0.2$. To define the correlation structure among longitudinal responses, we considered two types of correlations: $\operatorname{corr}\left(y_{i t}, y_{i t^{\prime}}\right)=\alpha^{\left|t-t^{\prime}\right|}$ for serial correlations and $\operatorname{corr}\left(y_{i t}, y_{i t^{\prime}}\right)=$ $\alpha$ for exchangeable correlations, for all $\left(t, t^{\prime}\right)$.

The correlated binary data were generated using a multivariate binary model suggested by Bahadur (1961). Model parameters were estimated by assuming both serial and exchangeable correlation structures. The association and regression parameters were fixed at $\alpha=0.5$ and $\left(\beta_{0}, \beta_{1}, \beta_{2}\right)=(-1,1,0.2)$, respectively, throughout the simulations. Each simulation run was based on 1000 replications of data 
sets, where each data set contained $k=500$ subjects and $T=3$ observations per subject.

\subsubsection{Dropout Models}

The dropout of a subject was assumed to depend on previous and current responses, that is,

$$
P\left(M_{i}=m \mid \mathbf{y}_{i}, \mathbf{X}_{i}, \boldsymbol{\tau}\right)=P\left(M_{i}=m \mid y_{i 1}, \ldots, y_{i m}, \boldsymbol{\tau}\right) .
$$

We assumed that all subjects were evaluated at the first time point, but dropouts occurred at second or third time points, so that $2 \leq M_{i} \leq 4$. For calculating $P\left(M_{i}=m \mid y_{i 1}, \ldots, y_{i m}, \boldsymbol{\tau}\right)$ in (3.46), the individual non-response probabilities were obtained from

$$
P\left(R_{i t}=0 \mid R_{i 1}=\cdots=R_{i t-1}=1, y_{i 1}, \ldots, y_{i t}, \boldsymbol{\tau}\right)=\frac{\exp \left(\psi_{i t}\right)}{1+\exp \left(\psi_{i t}\right)},
$$

where $\psi_{i t}=\tau_{0}+\tau_{1} y_{i t-1}+\tau_{2} y_{i t}$ for $\mathrm{t}=2$, 3. In this setting, $\tau_{2}=0$ corresponds to MAR, $\tau_{1}=\tau_{2}=0$ corresponds to MCAR, and $\tau_{2} \neq 0$ leads to non-ignorable (NI) missingness mechanism. Using (3.15) and (3.47) we get

$$
\nu_{i m}=P\left(M_{i}=m \mid y_{i 1}, \ldots, y_{i m}, \boldsymbol{\tau}\right)=\left\{\prod_{t=2}^{m-1} \frac{1}{1+\exp \left(\psi_{i t}\right)}\right\}\left\{\frac{\exp \left(\psi_{i m}\right)}{1+\exp \left(\psi_{i m}\right)}\right\}^{I\{m \leq T\}}
$$

where $\psi_{i t}=\tau_{0}+\tau_{1} y_{i, t-1}+\tau_{2} y_{i t}$ and $\psi_{i m}=\tau_{0}+\tau_{1} y_{i, m-1}+\tau_{2} y_{i m}$.

We ran our simulations for each combination of missing data model parameters $\boldsymbol{\tau}=\left(\tau_{0}, \tau_{1}, \tau_{2}\right)^{\prime}=(-2,3,0)^{\prime}$, and $\boldsymbol{\tau}=\left(\tau_{0}, \tau_{1}, \tau_{2}\right)^{\prime}=(-2,2,0)^{\prime}$ that correspond to MAR mechanism and also for $\boldsymbol{\tau}=\left(\tau_{0}, \tau_{1}, \tau_{2}\right)^{\prime}=(-2,3,0.5)^{\prime}$ and $\boldsymbol{\tau}=\left(\tau_{0}, \tau_{1}, \tau_{2}\right)^{\prime}=$ $(-2,3,1)^{\prime}$ that correspond to NMAR (NI) mechanism. Model parameters were estimated under misspecified missing data model (MAR). 


\subsubsection{Estimating Dropout Probabilities}

For the calculation of WGEE estimators, we solve iterative equations (3.24) and (3.25), where the response probability weights $\nu_{i m_{i}}$ are estimated from (3.48). In particular, missing data model parameters $\boldsymbol{\tau}$ are estimated by maximizing the likelihood

$$
\begin{gathered}
L(\boldsymbol{\tau})=\prod_{i=1}^{k} P\left(M_{i}=m_{i} \mid y_{i 1}, \ldots, y_{i m_{i}}, \boldsymbol{\tau}\right), \\
=\prod_{i=1}^{k}\left\{\prod_{t=2}^{m_{i}-1} \frac{1}{1+\exp \left(\psi_{i t}\right)}\right\}\left\{\frac{\exp \left(\psi_{i m_{i}}\right)}{1+\exp \left(\psi_{i m_{i}}\right)}\right\}^{I\left\{m_{i} \leq T\right\}},
\end{gathered}
$$

where $\psi_{i t}=\tau_{0}+\tau_{1} y_{i, t-1}+\tau_{2} y_{i t}$. Let

$$
\operatorname{logit}\left(P_{i t}^{*}(\boldsymbol{\tau})\right)=\tau_{0}+\tau_{1} y_{i, t-1}+\tau_{2} y_{i t}
$$

Then the score equation for estimating $\boldsymbol{\tau}$ is given by

$$
S(\boldsymbol{\tau})=\sum_{i=1}^{k}\left\{-\sum_{t=2}^{m_{i}-1} P_{i t}^{*}(\boldsymbol{\tau}) \mathbf{y}_{i t}^{*}+I\left\{m_{i} \leq T\right\}\left\{1-P_{i m_{i}}^{*}(\boldsymbol{\tau})\right\} \mathbf{y}_{i m_{i}}^{*}\right\}=0
$$

where $\mathbf{y}_{i t}^{*}=\left(1, y_{i, t-1}, y_{i t}\right)^{\prime}$. The variance of the estimator $\hat{\boldsymbol{\tau}}$ may be obtained from the information matrix

$$
I(\boldsymbol{\tau})=\sum_{i=1}^{k} \sum_{t=2}^{\min \left(m_{i}, T\right)} P_{i t}^{*}(\boldsymbol{\tau})\left(1-P_{i t}^{*}(\boldsymbol{\tau})\right) \mathbf{y}_{i t}^{*} \mathbf{y}_{i t^{\prime}}^{*}
$$

For the $i^{\text {th }}$ individual at time $t$, the predicted probability of response is obtained as

$$
1-\hat{P}_{i t}^{*}=\frac{1}{1+\exp \left(\mathbf{y}_{i t}^{\prime *} \hat{\boldsymbol{\tau}}\right)}
$$

Then for the $i^{\text {th }}$ individual at time $m_{i}$, the dropout out probability is estimated by

$$
\hat{\nu}_{i m_{i}}=P\left(M_{i}=m_{i} \mid y_{i 1}, \ldots, y_{i m_{i}}, \hat{\boldsymbol{\tau}}\right)=\left\{\prod_{t=2}^{m_{i}-1}\left(1-\hat{P}_{i t}^{*}\right)\right\} \times\left\{\hat{P}_{i m_{i}}^{*}\right\}^{I\left\{m_{i} \leq T\right\}}
$$




\subsubsection{Assessment Methods}

The aforementioned three methods were compared based on empirical biases, mean squared errors, and coverage probabilities of the estimators of regression and association parameters.

Bias: The empirical bias of an estimator $\hat{\theta}$ of $\theta$ is obtained as

$$
\operatorname{Bias}(\hat{\theta})=\frac{1}{S} \sum_{s=1}^{S}\left(\hat{\theta}_{s}-\theta\right)
$$

where $\hat{\theta}_{s}$ is the estimate of $\theta$ attained from the $s$ th simulated data set and $S$ is the simulation size.

The percentage relative bias is calculated as

$$
\frac{\operatorname{Bias}(\hat{\theta})}{\theta} \times 100
$$

Mean Squared Error: The empirical mean squared error (MSE) of $\hat{\theta}$ is obtained as

$$
\operatorname{MSE}(\hat{\theta})=E(\hat{\theta}-\theta)^{2} \approx \frac{1}{S} \sum_{s=1}^{S}\left(\hat{\theta}_{s}-\theta\right)^{2}
$$

Coverage Probability: The empirical coverage probability of $\hat{\theta}$ for a $95 \%$ confidence interval on $\theta$ is attained from

$$
\mathbf{C P}(\hat{\theta})=\frac{1}{S} \sum_{s=1}^{S} I\left\{\left|\hat{\theta}_{s}-\theta\right| \leq 1.96 \times S E\left(\hat{\theta}_{s}\right)\right\}
$$

where $S E(\hat{\theta})$ is an estimate of the standard error of $\hat{\theta}$ and $I$ is an indicator variable.

\subsubsection{Results}

Recall that in the first set of simulations, data were generated using the dropout model (3.48) with parameters $\boldsymbol{\tau}=\left(\tau_{0}, \tau_{1}, \tau_{2}\right)^{\prime}=(-2,2,0)^{\prime}$ and $(-2,3,0)^{\prime}$, which correspond to the MAR model. For these two sets of $\boldsymbol{\tau}$ parameters, the data contained approximately $30 \%$ and $40 \%$ missing values of the response variable, respec- 
tively. Tables 3.1 and 3.2 present empirical relative biases, mean squared errors and coverage probabilities of GEE, WGEE1 and WGEE2 estimates of the regression parameters $\boldsymbol{\beta}$ and the correlation parameter $\alpha$ under the above two MAR models.

It is clear from Tables 3.2 and 3.2 that the ordinary GEE method provides large percentage relative biases for the time effect $\hat{\beta}_{2}$ under all scenarios considered. The systematic biases of the GEE estimate $\hat{\beta}_{2}$ also increases when the proportion of missing data increases. For example, under the correctly specified serial correlation structure, when the percentage of missing data increases from $30 \%$ (at $\tau_{1}=2$ ) to $40 \%$ (at $\tau_{1}=3$ ), the absolute percentage relative bias of $\hat{\beta}_{2}$ increases from $18.35 \%$ to $59.40 \%$. The GEE method also provides large systematic biases for estimating the correlation parameter $\alpha$. For example, at $\tau_{1}=2$ the absolute percentage relative bias of the GEE estimate of $\alpha$ under the correctly specified serial correlation is obtained as $11.92 \%$, whereas at $\tau_{1}=3$, the corresponding percentage relative bias is obtained as $27.76 \%$.

When comparing the weighted GEE (WGEE) methods, both WGEE1 and WGEE2 provide approximately unbiased estimates of all the regression parameters $\left(\beta_{0}, \beta_{1}, \beta_{2}\right)$ under all scenarios considered. For estimating the correlation parameter $\alpha$, our proposed WGEE2 method generally provides unbiased estimates. On the other hand, WGEE1 method gives biased estimates of $\alpha$ under all scenarios. For example, at $\tau_{1}=3$ Table 3.1 shows that under the correctly specified exchangeable correlation structure, the WGEE1 method provides an absolute percentage relative bias of $17.32 \%$, whereas the WGEE2 method provides a very small bias of $0.34 \%$. The empirical mean squared errors of the estimates indicate that the WGEE2 method provides the most efficient estimates of the correlation parameter $\alpha$ under correctly specified correlation structures. Also, the WGEE2 method provides coverage probabilities that are generally close to the nominal 95\% confidence level. For example, when both the true and fitted models use the exchangeable correlation, the coverage probabilities of the estimates of $\alpha$ are obtained as $6.3 \%, 33.4 \%$ and $94.8 \%$ by the GEE, WGEE1 and WGEE2 methods, respectively, as shown in Table 3.1. The pro- 
posed WGEE2 method appears to provide robust estimates of the model parameters under misspecified correlation structures.

In the second set of simulations, data were generated using the non-ignorable (NI) dropout model (3.48) for each combination of $\boldsymbol{\tau}=(-2,3,0.5)^{\prime}$ and $\boldsymbol{\tau}=(-2,3,1)^{\prime}$. However, the fitted models still assumed the MAR dropout model. The goal of the study was to assess the robustness properties of the three methods under the misspecified missing data models as considered here. Tables 3.3 and 3.4 present empirical relative biases, mean squared errors and coverage probabilities of the estimates of regression and correlation parameters obtained under the three methods for $\boldsymbol{\tau}=(-2,3,0.5)^{\prime}$ and $\boldsymbol{\tau}=(-2,3,1)^{\prime}$, respectively.

It is clear from Tables 3.3 and 3.4 that under the non-ignorable dropout model, all three methods provide large systematic biases for estimating the time effect $\beta_{2}$. The GEE method appears to be the worst among these three methods with the largest bias, largest mean squared error and smallest coverage probability for estimating $\beta_{2}$. When estimating the correlation parameter $\alpha$, here the proposed WGEE2 method appears to be the most efficient. For example, under the correctly specified serial correlation structure, the WGEE2 estimate of $\alpha$ provides a coverage probability of $84.4 \%$, whereas the WGEE1 and ordinary GEE provide very poor empirical coverages of (Table 3.3) $21 \%$ and $0.5 \%$, respectively. Overall, the WGEE2 method provides the most robust estimates under misspecified dropout models.

The estimates of the parameters of MAR dropout models were obtained by maximizing the likelihood (3.50). Table 3.5 presents percentage relative biases and mean squared errors of the estimates of dropout model parameters $\left(\tau_{0}, \tau_{1}\right)$. It is clear that under the correctly specified MAR model, the estimates are generally unbiased, whereas under the misspecified NI missing data model, the ML method provides biased estimates of both $\tau_{0}$ and $\tau_{1}$, as expected. 


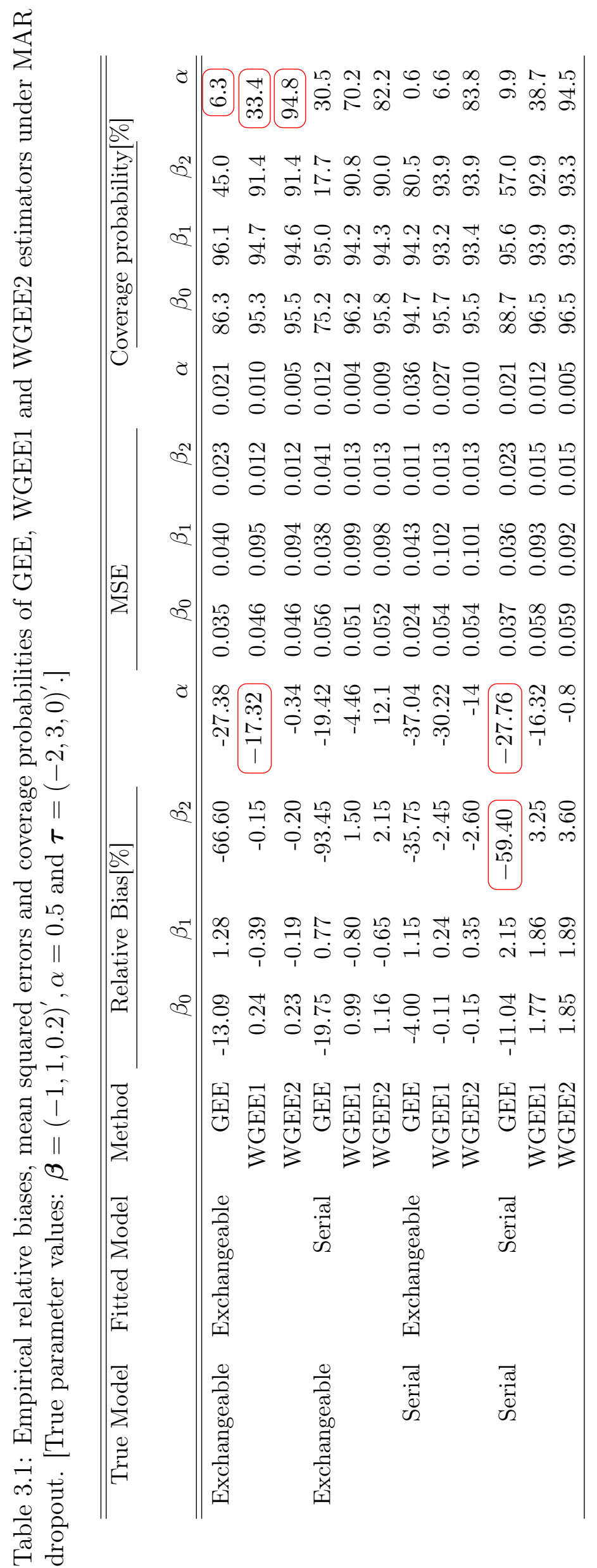




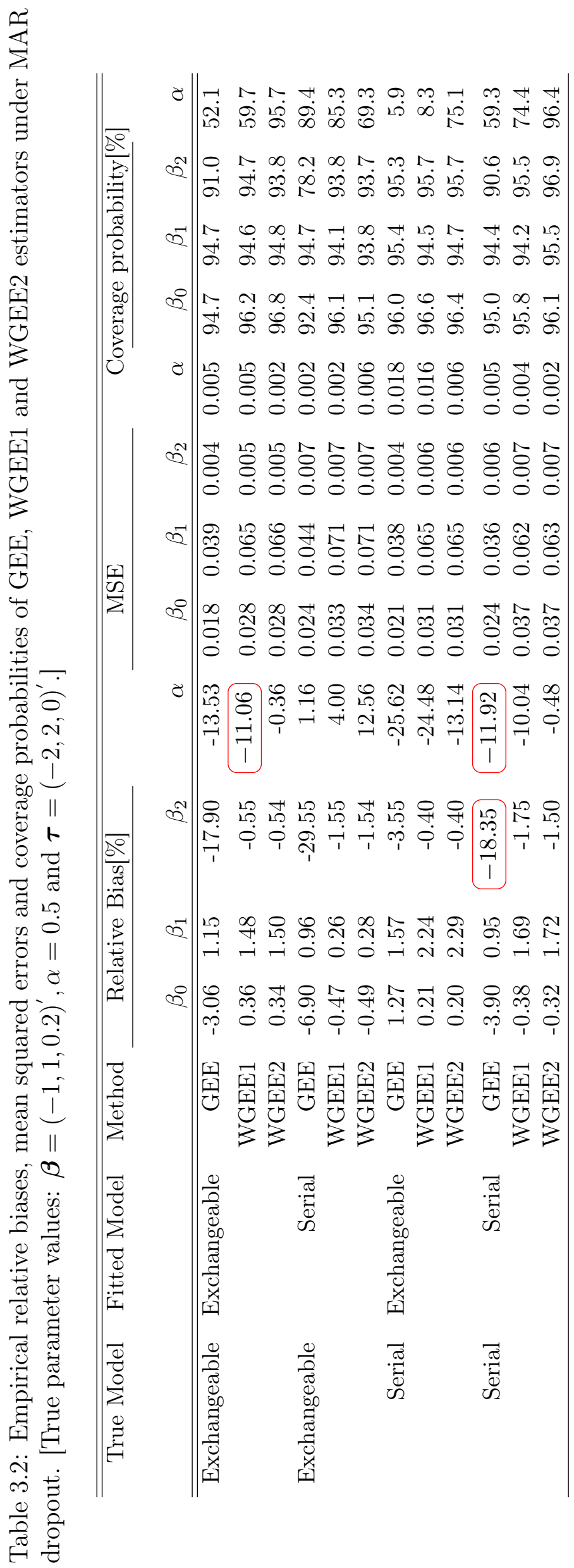




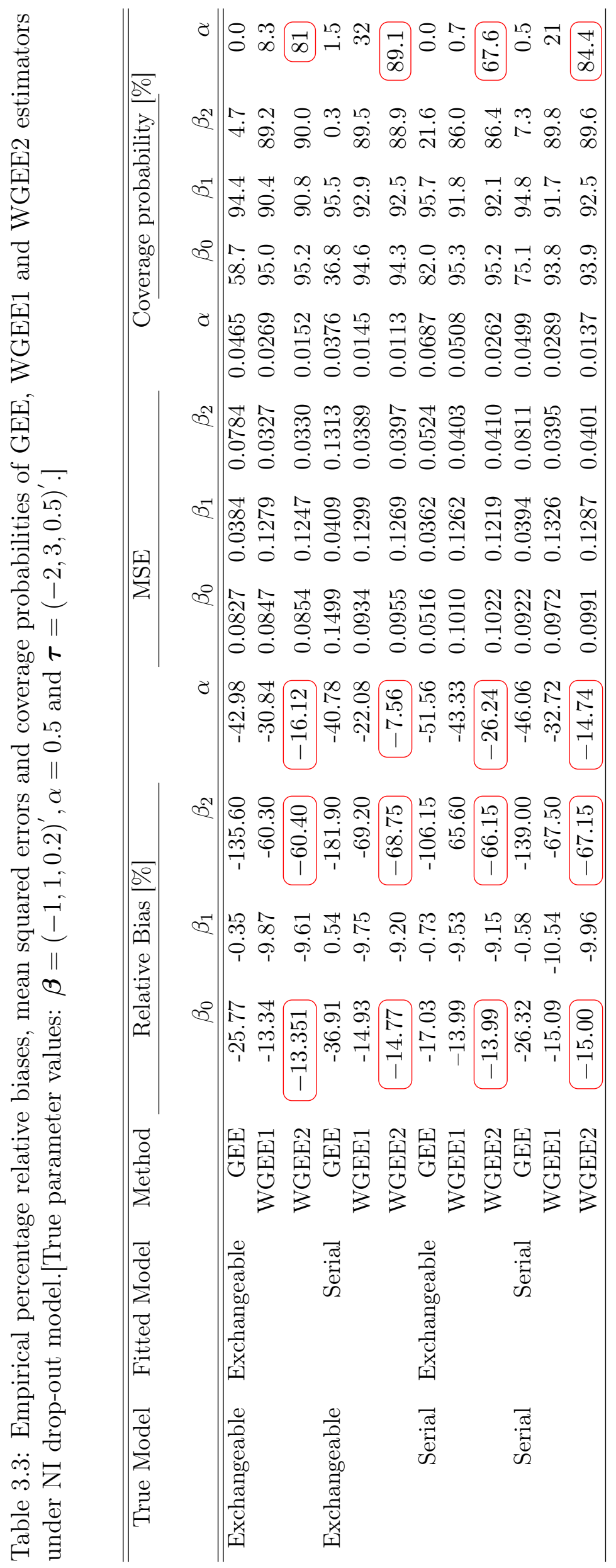




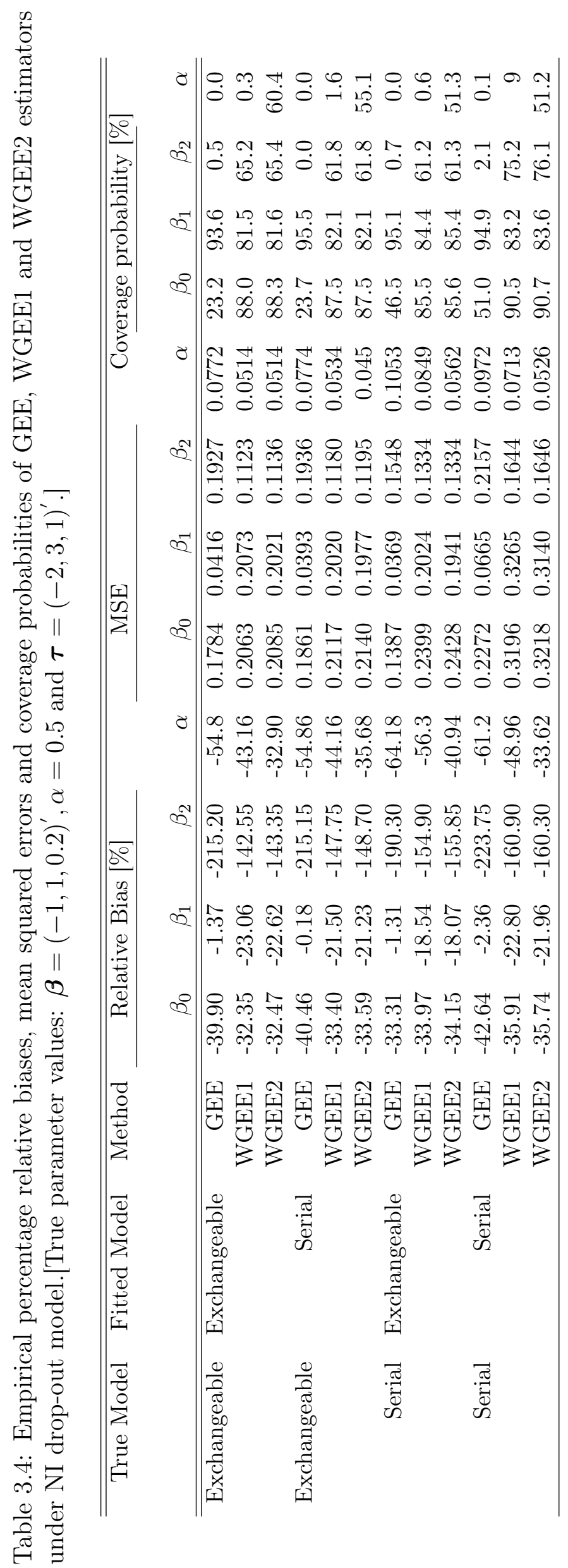


Table 3.5: Percentage relative biases and mean squared errors of maximum likelihood estimates under MAR and nonignorible dropout models.

MAR dropout Model for $k=500$

\begin{tabular}{|c|c|c|c|c|}
\hline \hline Correlation & Parameter & True value & Relative bias $\%$ & MSE \\
\hline \hline Exchangeable & $\tau_{0}$ & -2 & 0.53 & 0.0184 \\
& $\tau_{1}$ & 3 & 0.58 & 0.0361 \\
\hline \hline Serial & $\tau_{0}$ & -2 & 0.06 & 0.0165 \\
& $\tau_{1}$ & 3 & 0.51 & 0.0355 \\
\hline \hline Exchangeable & $\tau_{0}$ & -2 & 0.30 & 0.0176 \\
& $\tau_{1}$ & 2 & 0.43 & 0.0293 \\
\hline \hline Serial & $\tau_{0}$ & -2 & 0.38 & 0.0164 \\
& $\tau_{1}$ & 2 & 0.44 & 0.0309 \\
\hline
\end{tabular}

Non-Ignorable dropout Model for $\mathrm{k}=\mathbf{5 0 0}$

\begin{tabular}{|c|c|c|c|c|}
\hline \hline Correlation & parameter & True value & Relative bias $\%$ & MES \\
\hline \hline Exchangeable & $\tau_{0}$ & -2 & -13.84 & 0.0903 \\
& $\tau_{1}$ & 3 & 14.36 & 0.2325 \\
\hline \hline Serial & $\tau_{0}$ & -2 & -13.76 & 0.0899 \\
& $\tau_{1}$ & 3 & 14.62 & 0.2374 \\
\hline \hline Serial & $\tau_{0}$ & -2 & -14.04 & 0.0924 \\
& $\tau_{1}$ & 2 & 23.11 & 0.2444 \\
\hline \hline Exchangeable & $\tau_{0}$ & -2 & -13.21 & 0.0846 \\
& $\tau_{1}$ & 2 & 22.73 & 0.2373 \\
\hline \hline
\end{tabular}




\subsubsection{Application: Analysis of Smoking Data}

Here we consider data from an epidemiological research on Coronary Artery Risk Development in young adults (CARDIA), which investigates trend of cigarette smoking as potential cardiovascular risk factor among white and black females and males (Hughes et al., 1987). This research conducted in four metropolitan cities of the United States (Oakland, CA; Chicago, IL; Birmingham, AL, and Minneapolis, MN), where an aggregate of 5115 young grownups aged 18 - 30 years was prospectively followed and observed up to five times from 1986 to 1996. Recruitment, limited to white and black individuals, was designed to attain an estimated balance in size of a sample concerning gender, age, education, and race. The participants of this study were scheduled for appointments at $0,2,5,7$ and 10 years. We consider only the initial four appointments with a total of 5078 observations on the self-assessment status of smoking (yes/no) at baseline (year 0). We discarded a small fraction of data (578 out of 17995$)$ to obtain monotone missingness.

Let $y_{i t}=1$ if the the $i^{\text {th }}$ individual at the $t^{\text {th }}$ appointment is a smoker and $y_{i t}=0$, otherwise. Suppose $p_{i t}=E\left(y_{i t}\right)$ is the marginal mean response, which is defined as a function of covariates by

$$
\begin{aligned}
\operatorname{logit}\left(p_{i t}\right)=\beta_{0}+\beta_{1}(\text { age } / 10)_{i}+\beta_{2} \text { year }_{t} & +\beta_{3} \text { eduh }_{i}+\beta_{4} \text { educ }_{i} \\
& \\
& +\beta_{5} \text { racebf }_{i}+\beta_{6} \text { racewm }_{i}+\beta_{7} \text { racewf }_{i}
\end{aligned}
$$

for $i=1, \ldots, 5078$ and $t=1, \ldots, 4$, where $a g e_{i}=$ age of $i^{t h}$ individual in years at baseline time, year $_{t}=$ year since the measurements taken at baseline $=0,2,5$ and 7 , the binary indicator $e d u h_{i}=1$ if level of education of $i^{\text {th }}$ individual is high school or less, and 0 otherwise, $e d u c_{i}=1$ if level of education is up to some college, and 0 otherwise. If the individual is white female then indicator $\operatorname{racew}_{i}=1$, and 0 otherwise, if the individual is black female then $\operatorname{raceb}_{i}=1$, and 0 otherwise, and if the individual is white male then racewm $_{i}=1$, and 0 otherwise. 
For the estimation of missing data model parameters, we consider a model

$$
\operatorname{logit}\left(p_{i t}^{*}\right)=\tau_{0}+\tau_{1} y_{i, t-1}+\tau_{2} \text { racebf }_{i}+\tau_{3} \text { racewm }_{i}+\tau_{4} \text { racew }_{i}+\tau_{5} \text { year }_{t},
$$

where $p_{i t}^{*}=P\left(R_{i t}=0 \mid R_{i 1}=\ldots, R_{i, t-1}=1, \mathbf{y}_{i}, \mathbf{X}_{i}, \boldsymbol{\tau}\right)$ is the conditional probability that the $i^{\text {th }}$ individual drops out at time t.

Table 3.6 presents pseudo-ML estimates of $\boldsymbol{\tau}=\left(\tau_{0}, \ldots, \tau_{5}\right)^{\prime}$, their standard errors and corresponding z-values for testing individual parameters. Both smoking status and race appear to affect the response probabilities under the assumed MAR dropout model. For example, a young adult who currently smokes has $\exp (0.389)=1.47$ times greater odds to miss the next follow-up appointment, as compared to the one who does not smoke. Also, black males are more likely to miss a follow-up appointment, as compared to those from other race and gender.

The regression parameters $\boldsymbol{\beta}=\left(\beta_{0}, \ldots, \beta_{7}\right)^{\prime}$ were estimated using the three methods: GEE, WGEE1, and WGEE2. Table 3.7 presents estimates of $\boldsymbol{\beta}$ and $\alpha$ under the exchangeable correlation, and their corresponding standard error and z-values of the estimates. The estimates are also shown for the serial correlation structure in Table 3.7. The GEE estimates appear to be different than those obtained from the WGEE1 and WGEE2 methods. For example, for the covariate effects of age, the GEE method provides an estimate of 0.3221, whereas the WGEE1 and WGEE2 methods provides estimated values of 0.4824 and 0.4820 , respectively. The WGEE1 and WGEE2 estimates are generally close to each other. It appears that older young adults smoke more, but the smoking rate decreases over time. Education level also has an effect on the smoking trend; young adults with higher degrees are less likely to smoke. For example, the WGEE2 method under the serial correlation indicates that young adults with a high school degree or less have $\exp (1.7341)=5.664$ times higher odds of smoking, as compared to those with a college degree or more. Race is also an indicator of smoking; black males are more likely to smoke as compared to black females. The correlation coefficient $\alpha$ appears to be significant by all three methods. 


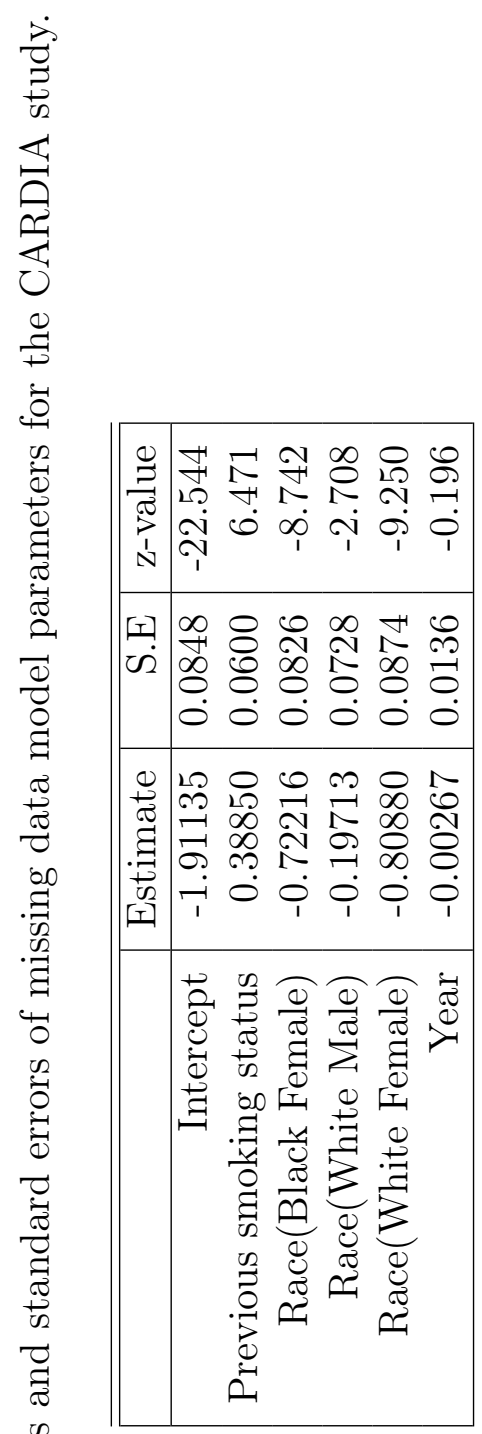

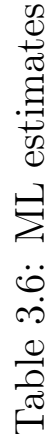

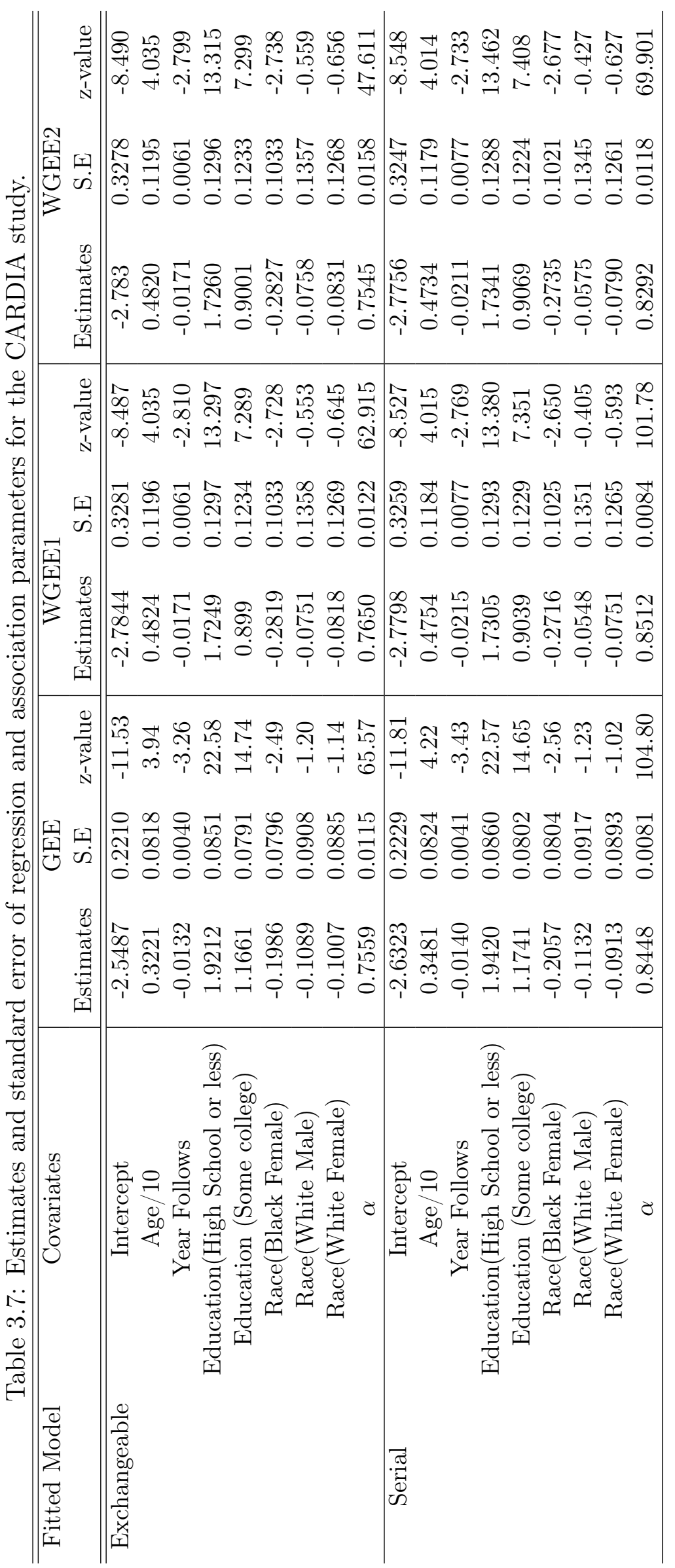




\subsection{Incomplete Count Longitudinal Model}

\subsubsection{Log Linear Response Model}

Let $y_{i t}$ be the count response of subject $i$ at visit $t$, and $\mathbf{x}_{i t}=\left(x_{i t, 1}, \ldots, x_{i t, p}\right)^{\prime}$ be a $p \times 1$ covariates vector for $i^{\text {th }}$ subject at time $t$. Throughout the observation time, the covariates may be considered fixed or time dependent. Assume the marginal distribution of the count response variable $y_{i t}$ is

$$
y_{i t} \sim \operatorname{Poisson}\left(\mu_{i t}\right), \quad i=1, \ldots, k, \quad t=1, \ldots, T
$$

The marginal mean and variance are given by

$$
\begin{gathered}
E\left(y_{i t} \mid \mathbf{X}_{i}\right)=\mu_{i t}, \\
\operatorname{var}\left(y_{i t} \mid \mathbf{X}_{i}\right)=\phi \mu_{i t},
\end{gathered}
$$

where $\phi$ is a dispersion parameter that needs to be estimated. Assume that the mean response $\mu_{i t}$ only depends on the covariates vector $\mathbf{x}_{i t}$ at time $t$ for subject $i$, so that $\mu_{i t}=E\left(y_{i t} \mid \mathbf{X}_{i}\right)=E\left(y_{i t} \mid \mathbf{x}_{i t}\right)$ (Sullivan Pepe and Anderson, 1994). We consider a log-linear model of the form

$$
\log \left(\mu_{i t}\right)=\mathbf{x}_{i t}^{\prime} \boldsymbol{\beta}
$$

where $\boldsymbol{\beta}=\left(\beta_{0}, \ldots, \beta_{p-1}\right)^{\prime}$ is a vector of regression parameters. Assume that responses $y_{i t}$ and $y_{i^{\prime}} t^{\prime}$ are uncorrelated when $i \neq i^{\prime}$. Let

$$
\operatorname{corr}\left(y_{i t}, y_{i t^{\prime}}\right)=\alpha_{t t^{\prime}}
$$

denote the correlation between $y_{i t}$ and $y_{i t^{\prime}}$ for given $\mathbf{x}_{i t}$, where $\boldsymbol{\alpha}=\left(\alpha_{12}, \ldots, \alpha_{1 T}\right.$, $\left.\alpha_{23}, \ldots, \alpha_{T-1, T}\right)^{\prime}$ is a vector of correlation parameters. Here the extra variance assumption of the Poisson model permits the variance to be inflated by a factor $\phi$ ( 
$\phi>1)$. The excess variability in count data can be accounted for by including the dispersion parameters $\phi$ (Fitzmaurice et al., 2012).

\subsubsection{Methods of Estimation}

\subsubsection{Generalized Estimating Equations}

For estimating the regression and association parameters, the methods of generalized estimating equation (GEE) and weighted generalized estimating equation (WGEE) are discussed in this section. Here the association parameters $\boldsymbol{\alpha}$, regression parameters $\boldsymbol{\beta}$ and dispersion parameter $\phi$ are of main interest, and $\boldsymbol{\tau}$ is considered as the nuisance parameters of the model for missing data. Partitioning $\boldsymbol{\mu}_{i}$ into $\boldsymbol{\mu}_{i}^{o}$ for observed data and $\boldsymbol{\mu}_{\boldsymbol{i}}{ }^{m}$ for unobserved data, we can write $\boldsymbol{\mu}_{i}=\left(\boldsymbol{\mu}_{i}^{o}, \boldsymbol{\mu}_{i}^{m}\right)$.

In GEE, longitudinal data are analyzed by ignoring the pattern of missing data, where parameters are estimated only from the observed data. To allow joint estimation of the regression and association parameters $(\boldsymbol{\beta}, \boldsymbol{\alpha})$, an extension of the GEE method was developed by Prentice (1988). Let $\boldsymbol{\vartheta}=\left(\boldsymbol{\alpha}^{\prime}, \phi\right)^{\prime}$ be the vector of association and dispersion parameters. We solve simultaneously the equations below for estimating the model parameters $\boldsymbol{\beta}, \boldsymbol{\alpha}$ and $\phi$. Specifically, for $\boldsymbol{\vartheta}$, we solve

$$
\mathbf{U}_{\boldsymbol{\vartheta}}(\boldsymbol{\beta}, \boldsymbol{\vartheta})=\sum_{i=1}^{k} \mathbf{L}_{i}^{\prime} \mathbf{W}_{i}^{-1}\left(\mathbf{r}_{i}^{o}-\boldsymbol{\delta}_{i}^{o}\right)=0
$$

where $\mathbf{L}_{i}=\frac{\partial \boldsymbol{\delta}_{i}^{o}}{\partial \vartheta}=\frac{\partial \boldsymbol{\delta}_{i}^{o}}{\partial(\boldsymbol{\alpha}, \phi)}, \boldsymbol{\delta}_{i}^{o}=E\left(\mathbf{r}_{i}^{o}\right)$, and $\mathbf{W}_{i}=\operatorname{var}\left(\mathbf{r}_{i}^{o}\right)$, with $\mathbf{r}_{i}^{o}$ being observed components of $\mathbf{r}_{i}=\left(r_{i 1} r_{i 2}, r_{i 1} r_{i 3}, \ldots, r_{i 1} r_{i T}, r_{i 2} r_{23}, \ldots, r_{i, T-1} r_{i, T}, r_{i 1}^{2}, r_{i 2}^{2}, \ldots, r_{i T}^{2}\right)^{\prime}$, with

$$
r_{i t}=\frac{\left(y_{i t}-\mu_{i t}\right)}{\sqrt{\mu_{i t}}} .
$$

The GEE estimators of $(\boldsymbol{\beta}, \boldsymbol{\vartheta})$ may be obtained by solving the following estimating equations

$$
\mathbf{U}_{\boldsymbol{\beta}}(\boldsymbol{\beta}, \boldsymbol{\vartheta})=\sum_{i=1}^{k} \mathbf{D}_{i}^{\prime} \mathbf{V}_{i}^{-1}\left(\mathbf{y}_{i}^{o}-\boldsymbol{\mu}_{i}^{o}\right)=0
$$




$$
\mathbf{U}_{\boldsymbol{\vartheta}}(\boldsymbol{\beta}, \boldsymbol{\vartheta})=\sum_{i=1}^{k} \mathbf{L}_{i}^{\prime} \mathbf{W}_{i}^{-1}\left(\mathbf{r}_{i}^{o}-\boldsymbol{\delta}_{i}^{o}\right)=0
$$

One can set $\mathbf{W}_{i}=\operatorname{var}\left(\mathbf{r}_{i}^{o}\right)$ to be the identity matrix to avoid estimating additional higher-order parameters and to reduce sampling variation (Diggle et al., 2002).

\subsubsection{Weighted Generalized Estimating Equations}

In the case of dropouts, the estimates of $\boldsymbol{\beta}, \boldsymbol{\vartheta}$ are obtained by using the weighted GEE method introduced by Robins et al. (1995). For given $\boldsymbol{\mu}^{o}$ and $\mathbf{y}^{o}$, the WGEE estimators of $\boldsymbol{\beta}$ and $\boldsymbol{\vartheta}$ are obtained by solving the weighted estimating equations

$$
\begin{aligned}
& \hat{\mathbf{U}}_{\boldsymbol{\beta}}(\boldsymbol{\beta}, \boldsymbol{\vartheta})=\sum_{i=1}^{k} \frac{1}{\nu_{i m}} \mathbf{D}_{i}^{\prime} \mathbf{V}_{i}^{-1}\left(\mathbf{y}_{i}^{o}-\boldsymbol{\mu}_{i}^{o}\right)=0, \\
& \hat{\mathbf{U}}_{\boldsymbol{\vartheta}}(\boldsymbol{\beta}, \boldsymbol{\vartheta})=\sum_{i=1}^{k} \frac{1}{\nu_{i m}} \mathbf{L}_{i}^{\prime} \mathbf{W}_{i}^{-1}\left(\mathbf{r}_{i}^{o}-\boldsymbol{\delta}_{i}^{o}\right)=0 .
\end{aligned}
$$

Since both estimating equations are unbiased for 0 , the WGEE estimators $(\hat{\boldsymbol{\beta}}, \hat{\boldsymbol{\vartheta}})$ are consistent for $(\boldsymbol{\beta}, \boldsymbol{\vartheta})$, as evident from the theory of standard method of moments. If dropout probabilities $\nu_{i m_{i}}$ are estimated consistently, then WGEE estimators would still provide consistent estimators of $(\boldsymbol{\beta}, \boldsymbol{\vartheta})$ (Robins et al., 1995).

To find the estimates of $(\boldsymbol{\beta}, \boldsymbol{\vartheta})$, the iterative procedure begins with some initial values $\left(\boldsymbol{\beta}_{s}, \boldsymbol{\vartheta}_{s}\right)$ and produces updated values $\left(\boldsymbol{\beta}_{s+1}, \boldsymbol{\vartheta}_{s+1}\right)$ from interim values $\left(\boldsymbol{\beta}_{s}, \boldsymbol{\vartheta}_{s}\right)$ by means of the iterative equations

$$
\begin{aligned}
& \boldsymbol{\beta}_{s+1}=\boldsymbol{\beta}_{s}+\left(\sum_{i=1}^{k} \frac{1}{\nu_{i m}} \mathbf{D}_{i}^{\prime} \mathbf{V}_{i}^{-1} \mathbf{D}_{i}^{\prime}\right)^{-1} \sum_{i=1}^{k} \frac{1}{\nu_{i m}} \mathbf{D}_{i}^{\prime} \mathbf{V}_{i}^{-1}\left(\mathbf{y}_{i}^{o}-\boldsymbol{\mu}_{i}^{o}\right), \\
& \boldsymbol{\vartheta}_{s+1}=\boldsymbol{\vartheta}_{s}+\left(\sum_{i=1}^{k} \frac{1}{\nu_{i m}} \mathbf{L}_{i}^{\prime} \mathbf{W}_{i}^{-1} \mathbf{L}_{i}^{\prime}\right)^{-1} \sum_{i=1}^{k} \frac{1}{\nu_{i m}} \mathbf{L}_{i}^{\prime} \mathbf{W}_{i}^{-1}\left(\mathbf{r}_{i}^{o}-\boldsymbol{\delta}_{i}^{o}\right),
\end{aligned}
$$

for $s=0,1,2, \ldots$, where each term on the right side is evaluated at the current estimates $\left(\boldsymbol{\beta}_{s}, \boldsymbol{\vartheta}_{s}\right)$. 


\subsubsection{Approximate Variance of WGEE Estimators}

According to White (1982), the variance covariance matrix of WGEE estimators $\hat{\boldsymbol{\beta}}$ and $\hat{\boldsymbol{\vartheta}}$ can be approximated by using sandwich type estimators. The variance of $\hat{\boldsymbol{\beta}}$ can be approximated from

$$
\mathrm{V}(\hat{\boldsymbol{\beta}})=\mathbf{H}_{\boldsymbol{\beta}}^{-1} \mathbf{O}_{\boldsymbol{\beta}} \mathbf{H}_{\boldsymbol{\beta}}^{-1}
$$

where $\mathbf{H}_{\boldsymbol{\beta}}=\sum_{i=1}^{k} \frac{1}{\nu_{i m}} \mathbf{D}_{i}^{\prime} \mathbf{V}_{i}^{-1} \mathbf{D}_{i}$ and $\mathbf{O}_{\boldsymbol{\beta}}=\sum_{i=1}^{k} \mathbf{S}_{\boldsymbol{\beta}, i} \mathbf{S}_{\boldsymbol{\beta}, i}^{\prime}$, with

$\mathbf{S}_{\boldsymbol{\beta}, i}=\frac{1}{\nu_{i m}} \mathbf{D}_{i}^{\prime} \mathbf{V}_{i}^{-1}\left(\mathbf{y}_{i}^{o}-\boldsymbol{\mu}_{i}^{o}\right)$. Similarly, variance of $\hat{\boldsymbol{\vartheta}}$ may be approximated by

$$
\mathbf{V}(\hat{\vartheta})=\mathbf{H}_{\vartheta}^{-1} \mathbf{O}_{\vartheta} \mathbf{H}_{\vartheta}^{-1}
$$

where $\mathbf{H}_{\boldsymbol{\vartheta}}=\sum_{i=1}^{k} \frac{1}{\nu_{i m}} \mathbf{L}_{i}^{\prime} \mathbf{W}_{i}^{-1} \mathbf{L}_{i}$ and $\mathbf{O}_{\vartheta}=\sum_{i=1}^{k} \mathbf{S}_{\boldsymbol{\vartheta}, i} \mathbf{S}_{\boldsymbol{\vartheta}, i}^{\prime}$,

with $\mathbf{S}_{\boldsymbol{\vartheta}, i}=\frac{1}{\nu_{i m}} \mathbf{L}_{i}^{\prime} \mathbf{W}_{i}^{-1}\left(\mathbf{r}_{i}^{o}-\boldsymbol{\delta}_{i}^{o}\right)$. The matrices $\mathbf{H}_{\boldsymbol{\vartheta}}, \mathbf{H}_{\boldsymbol{\beta}}$ and $\mathbf{O}_{\boldsymbol{\vartheta}}, \mathbf{O}_{\boldsymbol{\beta}}$ are evaluated at the WGEE estimators $\hat{\boldsymbol{\vartheta}}$ and $\hat{\boldsymbol{\beta}}$.

\subsubsection{Simulation Study}

We ran a series of simulations using incomplete longitudinal count data in order to study the empirical properties of GEE, WGEE1 and WGEE2 estimators, as described below:

1. GEE: Estimates of regression parameters $\boldsymbol{\beta}$, association parameters $\boldsymbol{\alpha}$ and dispersion parameter $\phi$ are attained by solving unweighted GEEs of Prentice (1988).

2. WGEE1: Estimates of regression parameters $\boldsymbol{\beta}$ are attained by solving weighted GEEs, but the estimates of association parameters $\boldsymbol{\alpha}$ and dispersion parameter $\phi$ are attained by solving unweighted GEEs.

3. WGEE2: Estimates of all parameters $(\boldsymbol{\beta}, \boldsymbol{\alpha}, \phi)$ are attained by solving weighted GEEs. 


\subsubsection{Generation of Correlated Counts}

To generate correlated count data, we use a Poisson mixed model in the form:

- $y_{i t} \mid b_{i} \sim$ ind.Poisson $\left(\mu_{i t}\right)$,

- $\log \left(\mu_{i t}\right)=\mathbf{x}_{i t}^{\prime} \boldsymbol{\beta}+b_{i}$,

- $b_{i} \sim \operatorname{Normal}\left(0, \sigma^{2}\right)$, for $i=1, \ldots, N$, and $\sigma=[0.05]$.

A large data set with $N=500,000$ subjects was generated from this mixed model. The GEE estimates under the exchangeable correlation were treated as the true population parameters. We then ran our simulations by generating random samples from this population with each combination of $k=300, k=500$ and $k=1000$ subjects with each subjects being measured at $T=3$ time points.

\subsubsection{Response Models}

The marginal mean response model is given by

$$
\log \left[E\left(y_{i t} \mid \mathbf{x}_{i t}, \boldsymbol{\beta}, \alpha, \phi\right)\right]=\beta_{0}+\beta_{1} x_{i}+\beta_{2} t+\beta_{3} x_{i} t
$$

for $i=1, \ldots, k$ and $t=1, \ldots, T$, where $x_{i}$ is a binary covariate specifying the treatment group with $P\left(x_{i}=1\right)=0.5$. The true values of parameters are $\boldsymbol{\beta}=$ $\left(\beta_{0}, \beta_{1}, \beta_{2}, \beta_{3}\right)^{\prime}=(1.60,-0.51,-0.50,-0.50)^{\prime}$ and $\boldsymbol{\vartheta}=(\alpha, \phi)^{\prime}=(0.20,1.30)^{\prime}$. Each set of simulations was based on 1000 replicates of data sets.

\subsubsection{Dropout Models}

We consider a dropout model, where the response probability depends on previous and current responses and is independent of the group indicator $x_{i}$. That is, we assume

$$
P\left(M_{i}=m \mid \mathbf{y}_{i}, \mathbf{X}_{i}, \boldsymbol{\tau}\right)=P\left(M_{i}=m \mid y_{i 1}, \ldots, y_{i m}, \boldsymbol{\tau}\right) .
$$


We assume that all subjects are observed at the first time point. We denote $R_{i t}=1$ for observed $y_{i t}$ and 0 if $y_{i t}$ is missing. The dropout probability is given by

$$
P\left(R_{i t}=0 \mid R_{i 1}=\cdots=R_{i t-1}=1, y_{i 1}, \ldots, y_{i t-1}, y_{i t}, \boldsymbol{\tau}\right)=\frac{\exp \left(\psi_{i t}\right)}{1+\exp \left(\psi_{i t}\right)},
$$

where $\psi_{i t}=\tau_{0}+\tau_{1} y_{i t-1}+\tau_{2} y_{i t}$, for $t=2,3$. Note that we have $\tau_{2}=0$ for MAR, and, $\tau_{2} \neq 0$ leads us to NI(non-ignorable) missing data model, where missingness depends on the current response. Using (3.15) and (3.73), we get

$$
\nu_{i m}=P\left(M_{i}=m \mid y_{i 1}, \ldots, y_{i m}, \boldsymbol{\tau}\right)=\left\{\prod_{t=2}^{m-1} \frac{1}{1+\exp \left(\psi_{i t}\right)}\right\}\left\{\frac{\exp \left(\psi_{i m}\right)}{1+\exp \left(\psi_{i m}\right)}\right\}^{I\{m \leq T\}} .
$$

To measure the performance of our proposed methods WGEE1 and WGEE2 under missing data and to compare these with the ordinary second-order GEE approach of Prentice (1988), two sets of simulations were performed. In the first set, data were produced under "TRUE MAR" model, where the missing data parameters were considered as $\boldsymbol{\tau}=(-1.5,0.3,0)^{\prime}$, while the model parameters were also estimated under "TRUE MAR" model. In the second set, data were generated under "TRUE NI" missing data model, where $\boldsymbol{\tau}=(-1.5,0.3,0.3)^{\prime}$, and the model parameters were estimated under the misspecified NI model.

A sequence of 1000 replicates of data sets were produced for each combination of missing data parameters $\boldsymbol{\tau}=(-1.5,0.3,0)^{\prime}$ and $\boldsymbol{\tau}=(-1.5,0.3,0.3)^{\prime}$, and for $k=300,500$ and 1000 subjects observed at $T=3$ time points. Both cases produced approximately $32 \%$ missing data on the response variable.

\subsubsection{Estimating Dropout Probabilities}

To find WGEE estimators, the response probability weights $\nu_{i m_{i}}$ are estimated by using equation (3.15). The pseudo-likelihood function for $\boldsymbol{\tau}$ is given by

$$
L(\boldsymbol{\tau})=\prod_{i=1}^{k} P\left(M_{i}=m_{i} \mid y_{i 1}, \ldots, y_{i m_{i}}, \boldsymbol{\tau}\right)
$$




$$
=\prod_{i=1}^{k}\left\{\prod_{t=2}^{m_{i}-1} \frac{1}{1+\exp \left(\psi_{i t}\right)}\right\}\left\{\frac{\exp \left(\psi_{i m_{i}}\right)}{1+\exp \left(\psi_{i m_{i}}\right)}\right\}^{I\left\{m_{i} \leq T\right\}},
$$

where $\psi_{i t}=\tau_{0}+\tau_{1} y_{i, t-1}+\tau_{2} y_{i t}$. Let

$$
\operatorname{logit}\left(P_{i t}^{*}(\boldsymbol{\tau})\right)=\tau_{0}+\tau_{1} y_{i, t-1}+\tau_{2} y_{i t}
$$

From (3.75), the score equation for $\boldsymbol{\tau}$ take the form

$$
S(\boldsymbol{\tau})=\sum_{i=1}^{k}\left\{-\sum_{t=2}^{m_{i}-1} P_{i t}^{*}(\boldsymbol{\tau}) \mathbf{y}_{i t}^{*}+I\left\{m_{i} \leq T\right\}\left\{1-P_{i m_{i}}^{*}(\boldsymbol{\tau})\right\} \mathbf{y}_{i m_{i}}^{*}\right\}=0
$$

where $\mathbf{y}_{i t}^{*}=\left(1, y_{i, t-1}, y_{i t}\right)^{\prime}$. The pseudo-ML estimator $\hat{\boldsymbol{\tau}}$ is obtained by solving (3.76) using an iterative method. The variance of $\hat{\tau}$ may be approximated from the information matrix

$$
I(\boldsymbol{\tau})=\sum_{i=1}^{k} \sum_{t=2}^{\min \left(m_{i}, T\right)} P_{i t}^{*}(\boldsymbol{\tau})\left(1-P_{i t}^{*}(\boldsymbol{\tau})\right) \mathbf{y}_{i t}^{*} \mathbf{y}_{i t^{\prime}}^{*}
$$

For the $i^{t h}$ individual at time $t$, the predicted probability of response is obtained as

$$
1-\hat{P}_{i t}^{*}=\frac{1}{1+\exp \left(\mathbf{y}_{i t}^{\prime *} \hat{\boldsymbol{\tau}}\right)}
$$

Then for the $i^{\text {th }}$ individual at time $m_{i}$, the dropout probability is estimated by

$$
\hat{\nu}_{i m_{i}}=P\left(M_{i}=m_{i} \mid y_{i 1}, \ldots, y_{i m_{i}}, \boldsymbol{\tau}\right)=\left\{\prod_{t=2}^{m_{i}-1}\left(1-\hat{P}_{i t}^{*}\right)\right\} \times\left\{\hat{P}_{i m_{i}}^{*}\right\}^{I\left\{m_{i} \leq T\right\}}
$$

We solve (3.65) and (3.66) by replacing $\nu_{i m}$ with $\hat{\nu}_{i m}$ for the WGEE estimators $\hat{\boldsymbol{\beta}}$ and $\hat{\vartheta}$.

\subsubsection{Results}

Table 3.8 presents empirical percentage relative biases, mean squared errors and coverage probabilities of GEE, WGEE1 and WGEE2 estimators of the regression parameters $\boldsymbol{\beta}=\left(\beta_{0}, \beta_{1}, \beta_{2}, \beta_{3}\right)^{\prime}$, the association and dispersion parameters $(\alpha, \phi)$ 
under the correctly specified MAR model with $\boldsymbol{\tau}=(-1.5,0.3,0)^{\prime}$. Table 3.9 repeats the results for misspecified missing data model with $\boldsymbol{\tau}=(-1.5,0.3,0.3)^{\prime}$. It is clear from Table 3.8 that under the MAR model, both WGEE1 and WGEE2 methods provide close results for estimating the regression parameters. For estimating the correlation parameter $\alpha$ and dispersion parameter $\phi$, the proposed WGEE2 method appears to be the most efficient in terms of small percentage relative biases and good coverage probabilities. For example, when estimating $\alpha$ at $k=500$, the WGEE1 method provides a percentage relative bias of $-17.40 \%$ and a coverage probability of $81.1 \%$, whereas the WGEE2 method provides a much smaller percentage relative bias of $6.25 \%$ and a better coverage probability of $94.6 \%$. Also, for estimating $\phi$ at $k=500$, the WGEE1 method provides a percentage relative bias of $-13.24 \%$ and a poor coverage probability of $40.9 \%$, whereas WGEE2 estimator provides a very smaller percentage relative bias of $0.65 \%$ and good coverage probability of $95.1 \%$ for the nominal $95 \%$ confidence level.

Under the misspecified dropout model, Table 3.9 indicates that all three methods provide biased estimates of the regression parameters $\left(\beta_{0}, \beta_{1}, \beta_{2}, \beta_{3}\right)$ and association parameters. However the extent of the bias from the WGEE2 method is generally less as compared to the other two method. For example, when estimating $\alpha$ at $k=$ 1000, the WGEE2 method provides a bias of $-24.7 \%$, whereas the WGEE1 and GEE methods provide biases of $-43.6 \%$ and $-44.6 \%$, respectively. For the overdispersion parameter $\phi$, unlike the GEE and WGEE1 methods, the WGEE2 method generally provides unbiased estimates and a coverage probability that is close to the nominal confidence level. Overall, the WGEE2 method appears to provide the most robust and efficient estimators, as compared to the other two methods considered here.

Table 3.10 presents empirical relative biases and mean squared errors of the dropout model parameters $\left(\tau_{o}, \tau_{1}\right)$ under correctly specified MAR and misspecified NMAR models, respectively. As expected, the pseudo-ML estimates are unbiased under the MAR model. But under the misspecified (NI) model, Table 3.10 indicates systematic bias in the estimates of both $\tau_{o}$ and $\tau_{1}$. 


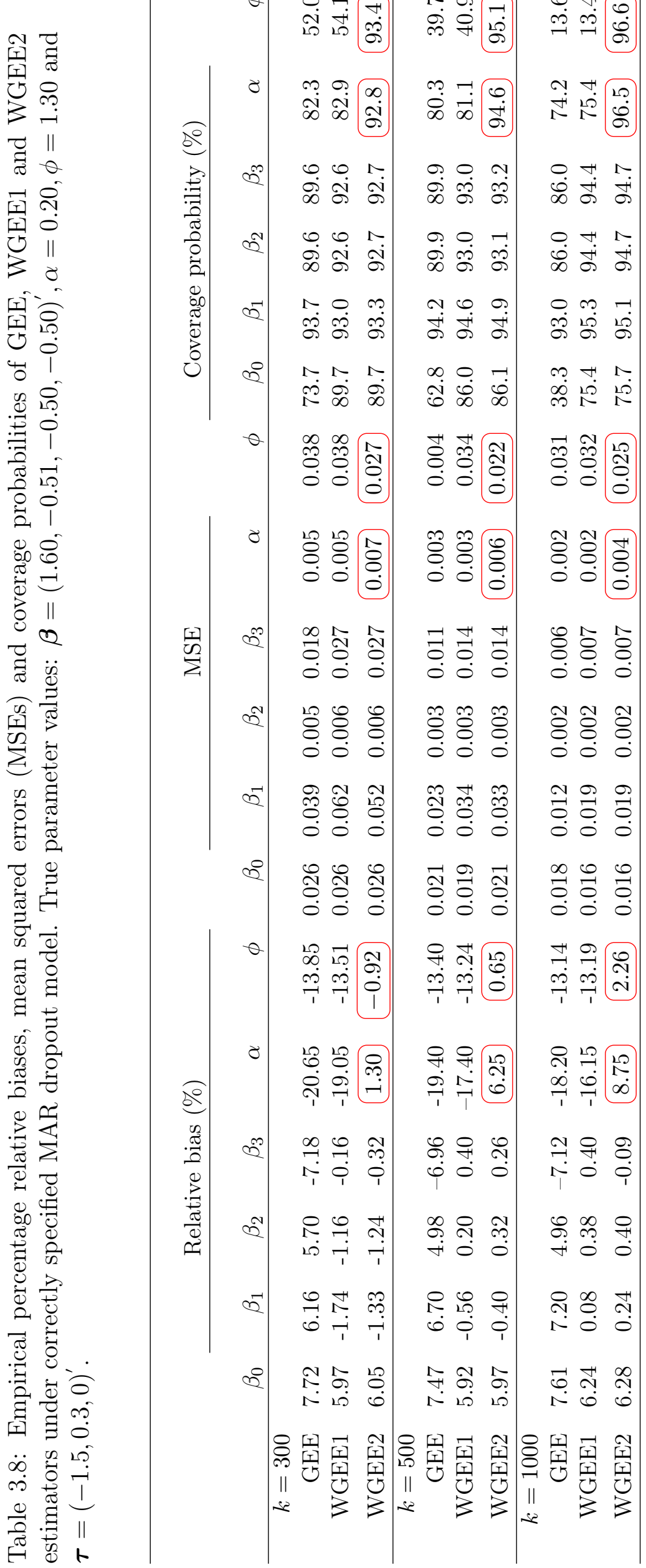




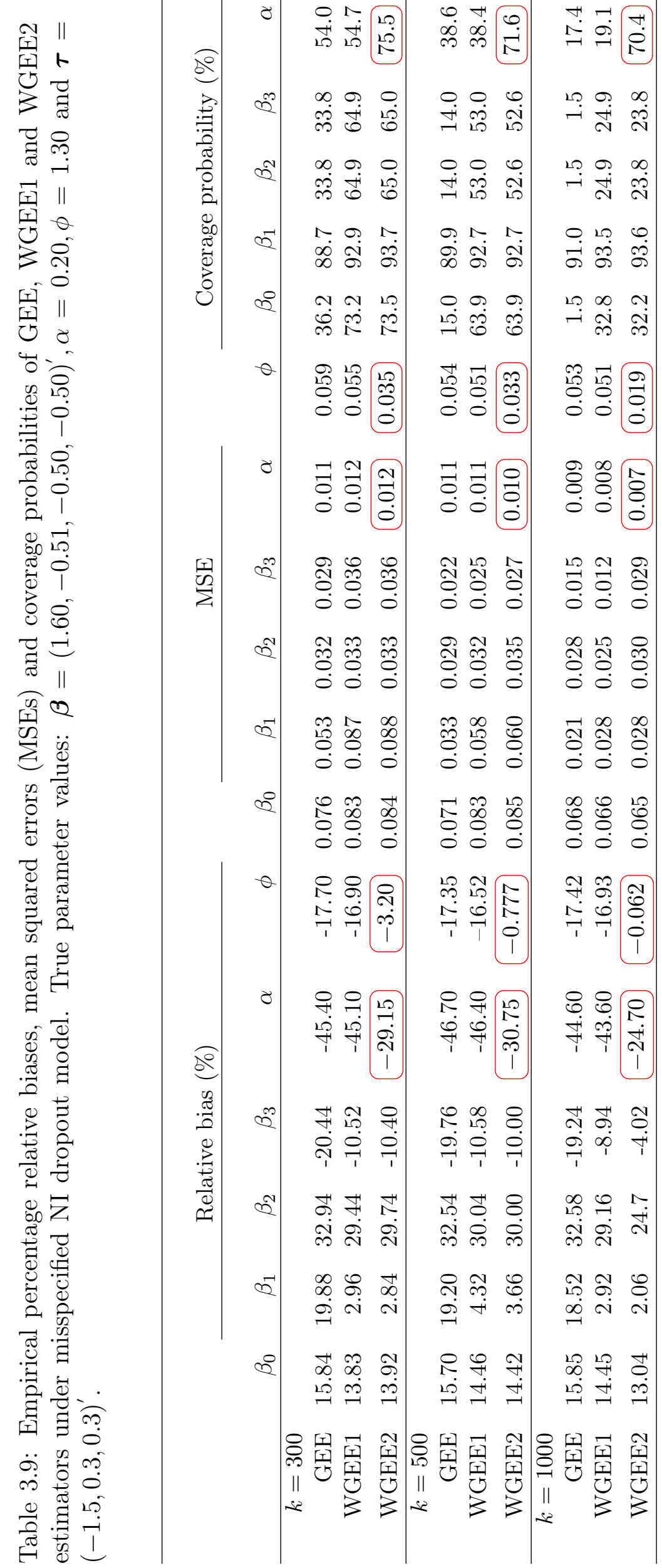


Table 3.10: Empirical relative biases and mean squared errors of maximum likelihood estimates under MAR and nonignorible dropout models.

MAR dropout Model

\begin{tabular}{|c|c|c|c|c|}
\hline \hline Sample Size & Parameter & True value & Relative bias\% & MSE \\
\hline \hline \multirow{2}{*}{$k=300$} & $\tau_{0}$ & -1.5 & 0.53 & 0.0219 \\
\hline \hline$k=500$ & $\tau_{1}$ & 0.3 & 0.60 & 0.0027 \\
\hline \hline & $\tau_{0}$ & -1.5 & 0.14 & 0.0138 \\
$k=1000$ & $\tau_{1}$ & 0.3 & 0.41 & 0.0027 \\
\hline \hline
\end{tabular}

Non-Ignorable dropout Model

\begin{tabular}{|c|c|c|c|c|}
\hline \hline Sample Size & parameter & True value & Relative bias $\%$ & MES \\
\hline \hline & $\tau_{0}$ & -1.5 & -8.48 & 0.0388 \\
$k=300$ & $\tau_{1}$ & 0.3 & 32.67 & 0.0132 \\
\hline \hline$k=500$ & $\tau_{0}$ & -1.5 & -8.33 & 0.0289 \\
$k$ & $\tau_{1}$ & 0.3 & 32.16 & 0.0113 \\
\hline \hline$k=1000$ & $\tau_{0}$ & -2 & -14.04 & 0.0924 \\
\hline \hline
\end{tabular}




\subsection{Conclusion}

The aim of this research was to provide a better alternative to the GEE approach of Prentice (1988) for analyzing missing longitudinal data. Our simulation study reveals that the suggested WGEE2 method typically offers unbiased and efficient estimators if a MAR model is accompanied by the missing data process. The three approaches offer biased estimators of the model parameters when the missing data are non-ignorable. The degree of the bias from the WGEE2 approach is usually less extreme relative to the unweighted approaches of GEE and WGEE1. 


\section{Chapter 4}

\section{Semiparametric Longitudinal Model}

\subsection{Introduction}

There has been substantial interest in expanding semiparametric and nonparametric regression techniques for longitudinal/clustered data. A complete nonparametric regression undergoes from the "curse of dimensionality", and to create effective statistical analysis certain forms on regression function is generally established. Semiparametric or partially linear models deal with the problem of dimensionality by presuming certain parametric forms. In a partially linear model, the mean response is assumed to rely on certain covariates $\mathbf{X}$ parametrically and certain additional covariates $\mathbf{Z}$ nonparametrically. This model is specifically interesting when the effects of $\mathbf{X}$ (e.g., treatments) are of main concern, whereas the effects of $\mathbf{Z}$ (e.g., confounders) are irritant parameters. Effective estimation for partially linear models is studied broadly and is interpretable for independent data; see, for example, Martinussen and Scheike (2002). Severini and Staniswalis (1994) and Speckman (1988) reflected partially linear models in the perspective of Cox regression model for survival data.

In the case of clustered data, for a semiparametric model, the main concern is how to successfully lay hold of within-cluster correlation. When parametric covariates are dependent on a nonparametric covariate, an ordinary implementation of the 
polynomial technique cannot appropriately account for within-cluster correlation and consequently fails to produce a semiparametric effectual estimator (Lin and Carroll, 2001a). On the other hand, a similar form of core method can be considered for within cluster correlation and can create an effective estimator in a case when a covariate displaying nonparametrically is a covariate of cluster level, as a family level covariate in familial studies or a time independence covariate in longitudinal studies (Lin and Carroll, 2001b).

The purpose of this research was to suggest a simple, joint technique that is effective in semiparametric regression irrespective of whether or not the covariate displaying nonparametrically is of cluster level. The proposed technique is centered on a spline method for estimating the nonparametric part and the adoption of usual generalized estimating equations (GEEs). The spline-based method is known to provide optimal convergence rates in a semiparametric model for both parametric and nonparametric components (Heckman 1986; He and Shi 1996). The proposed estimation of both nonparametric and parametric parts of the model has the advantage of possessing similar good characteristics as to that of the parametric GEE, i.e., the approximations are effectual if the covariance structure is properly stated, and are still stable and asymptotic normal even if the covariance structure is misspecified. The effectiveness of the semiparametric approximation is attained by supposing only conditional moment limitations and the strict Gaussian error assumption is not required. We adopt the second-order GEE approach of Prentice (1988) to analyze longitudinal responses under semiparametric models, where the nonlinear function is approximated by the spline regression.

This chapter is structured as follows. Section 4.2 introduces the model and notation to define the semiparametric marginal model for longitudinal data. Section 4.3 reviews the regression spline methods. Section 4.4 introduces a logistic partially linear response model for binary longitudinal data, while methods of estimation are discussed in Section 4.4.2. The asymptotic properties of the proposed GEE estimators are discussed in Section 4.4.3, and Section 4.4.4 examines the simulation 
study in case of a partially linear binary response model.

For count longitudinal data, Section 4.5 gives a brief introduction of log-linear and partially log-linear models. Section 4.5.2 reviews the proposed method of approximation, while the finite sample properties of the proposed estimators are studied in Section 4.5.3 based on simulations. Section 4.6 gives a brief conclusion of the chapter.

\subsection{Semiparametric Marginal Model}

In this section, the semiparametric regression model for longitudinal data is presented. Let the data comprise of $k$ subjects $(i=1, \ldots, k)$ with each subject consisting of a fixed set of $T$ observations. Let $y_{i t}$ be the response variable and $\left(\mathbf{x}_{i t}, z_{i t}\right)$ the covariates from the $i^{t h}$ subject observed at the $t^{t h}(t=1, \ldots, T)$ timepoint, where $\mathbf{x}_{i t}$ and $z_{i t}$ are a $p \times 1$ vector and a scalar, respectively. Given the covariates $\left(\mathbf{x}_{i t}, z_{i t}\right)$, the marginal mean and variance of the outcome variable $y_{i t}$ are given by

$$
E\left(y_{i t} \mid \mathbf{x}_{i t}, z_{i t}\right)=\mu_{i t}
$$

and

$$
\operatorname{var}\left(y_{i t} \mid \mathbf{x}_{i t}, z_{i t}\right)=\phi v\left(\mu_{i t}\right)
$$

The marginal mean $\mu_{i t}$ relies on $\mathbf{x}_{i t}$ and $z_{i t}$ by a known differentiable and monotonic link function $f($.$) , given by$

$$
\eta_{i t}=f\left(\mu_{i t}\right)=\mathbf{x}_{i t}^{\prime} \boldsymbol{\beta}+m\left(z_{i t}\right), \quad \mu_{i t}=f^{-1}\left(\eta_{i t}\right),
$$

where $\boldsymbol{\beta}$ is $p \times 1$ vector of regression coefficient and $m($.$) is an unidentified smooth$ function. The fundamental supposition is that without loss of generality $z_{i t}$ are all scaled into the interval $[0,1]$ and the observational values from subjects or clusters of different natures are independent.

The effect of covariates $\mathbf{x}$ are modeled parametrically and the effects of $\mathrm{z}$ is modeled 
nonparametrically. Model (4.3) varies from standard GEE models (Liang and Zeger, 1986) essentially through the existence of the nonparametric component $m($.$) . It is$ inspired by the reality that the effect of covariate $\mathrm{z}$ may be complex and might be well modeled nonparametrically (Zeger and Diggle, 1994). It is noted that our model has no distributional assumptions on the data other than the conditions of the moments specified in (4.1) and (4.2). In particular, $\mathbf{x}$ and $\mathrm{z}$ are allowed to be dependent, as usually seen for longitudinal/clustered data. It is important to note the assumption (Pepe and Couper, 1997) that

$$
E\left(y_{i t} \mid \mathbf{X}_{i}, \mathbf{Z}_{i}\right)=E\left(y_{i t} \mid \mathbf{x}_{i t}, z_{i t}\right)
$$

In matrix notation, we have

$$
\boldsymbol{\eta}_{i}=f\left(\boldsymbol{\mu}_{i}\right)=\mathbf{X}_{i} \boldsymbol{\beta}+m\left(\mathbf{Z}_{i}\right)
$$

where $\boldsymbol{\mu}_{i}=\left(\mu_{i 1}, \ldots, \mu_{i T}\right)^{\prime}, \mathbf{y}_{i}=\left(y_{i 1}, \ldots, y_{i T}\right)^{\prime}$, and $\mathbf{X}_{i}=\left(\mathbf{x}_{i 1}, \ldots, \mathbf{x}_{i T}\right)^{\prime}, \mathbf{Z}_{i}=$

$\left(z_{i 1}, \ldots, z_{i T}\right)^{\prime}$. If model (4.5) does not comprise $\mathbf{X}_{i} \boldsymbol{\beta}$, then it leads to the nonparametric model considered by Lin and Carroll (2000). Severini and Staniswalis (1994) examined a model analogous to (4.1)-(4.3). It is important to note that the marginal model for clustered data provides over-identification of the mean and variance functions. This is in the essence of GEE type models (Liang and Zeger, 1986). Apart from Gaussian data, the proposed marginal models need not be complete semiparametric likelihood descriptions.

\subsection{Regression Spline Methods}

Wold (1974), Smith (1979) and Eubank (1999) define the spline regression as piecewise polynomials, which are identified by a set of divisions (knots) and certain conditions of continuity. Spline regression can also be defined as the linear combination of a spline regression basis. Eubank (1999) introduced the truncated basis and 
De Boor (1978) introduced the B-spline basis, which are considered the two most commonly used spline regression basis functions. Ruppert and Carroll (2000) used truncated polynomials as basis functions, while He et al. (2002), He et al. (2005), Huang et al. (2007) and Lin et al. (2017) among others used B-spline basis functions. Spline regression is an important technique used to smooth the longitudinal data as this is a simple extension of polynomials.

Regression splines are of different types:

1. Naïve regression splines (Huggins and Loesch, 1998 and Huang et al., 2002),

2. Generalized regression splines (Wang, 1998 and Zhang, 1997),

3. Mixed effect regression splines (Shi et al., 1996 and Zhang, 1997).

The objective of the first two techniques is to estimate a function of a population mean for a set of longitudinal data, whereas the third technique is used to estimate the function of a population mean and also separable functions at the same time.

This study emphasizes the second kind of spline regression with a truncated power basis function. Spline regression can be set up by using the following $l^{\text {th }}$ degree truncated power basis on $q$ inner knots $\left(0=\zeta_{0}<\zeta_{1}<\zeta_{2}<\cdots<\zeta_{q}<\zeta_{q+1}=1\right)$ as:

$$
\Phi_{l, q}(z)=\left[1, z, \ldots, z^{l},\left(z-\zeta_{1}\right)_{+}^{l}, \ldots,\left(z-\zeta_{q}\right)_{+}^{l}\right]^{\prime}
$$

where $w^{l}=\left[w_{+}\right]^{l}$ denotes power $l$ of the positive part of $w$ with $w_{+}=\max (0, w)$. Note that the basis function consists of the first $(l+1)$ components for polynomials of degree $l$ and the rest are truncated power functions of degree $l$; usually truncated power basis (4.6) of degree " $l=0,1,2,3$ " is called "constant, linear, quadratic and cubic" truncated power basis, respectively. Therefore, using the truncated power basis (4.6), the spline regression can be expressed as:

$$
s(z)=\sum_{j=0}^{l} \gamma_{j} z^{j}+\sum_{r=1}^{q} \gamma_{l+r}\left(z-\zeta_{r}\right)_{+}^{l},
$$

where $\gamma_{0}, \gamma_{1}, \ldots, \gamma_{l+q}$ are the associated coefficients. The above equation means that, a) $s($.$) is a piecewise polynomial of order l$ on sub interval $\left[\zeta_{r}, \zeta_{r+1}\right)$, 
b) $s($.$) has l-1$ continuous derivatives and

c) $s($.$) has l$ discontinuous derivatives with jumps at $\zeta_{1}, \ldots \zeta_{q}$ (Eubank, 1999). For convenience, it may be called a regression spline of degree $l$ with knots $\zeta_{1}, \ldots, \zeta_{q}$. The regression spline (4.7) approximates the unknown smooth function $m(z)$ by

$$
\begin{aligned}
m(z) & \approx \sum_{j=0}^{l} \gamma_{j} z^{j}+\sum_{r=1}^{q} \gamma_{l+r}\left(z-\zeta_{r}\right)_{+}^{l} \\
& =\Phi_{l, q}^{\prime}(z) \gamma
\end{aligned}
$$

where $\gamma=\left(\gamma_{0}, \gamma_{1}, \ldots, \gamma_{l+q}\right)^{\prime}$. B-splines and truncated polynomials are linked directly, as B-splines can be seen as properly scaled $(l+1)^{\text {th }}$ order divided differences of the truncated polynomials (De Boor, 1978).

Our proposed estimation approach is based on the spline approximation (4.8), where $\mathrm{m}($.$) is a smooth function. If m($.$) is continuous, the spline approximation$ can be taken to satisfy $\sup _{z}\left|m(z)-\Phi_{l, q}(z)^{\prime} \gamma\right| \rightarrow 0$ as $N_{q}=(l+q) \rightarrow \infty$ (De Boor, 1978). Thus the function $\mathrm{m}(\cdot)$ in (4.8) is estimated by the spline regression, where the standard GEE is applied to estimate $\gamma$. Letting $\Phi\left(z_{i t}\right)=\Phi_{i t}$, the linear predictor (4.3) is approximated by

$$
\eta_{i t}=f\left(\mu_{i t}\right)=\mathbf{x}_{i t}^{\prime} \boldsymbol{\beta}+\Phi_{i t}^{\prime} \boldsymbol{\gamma}
$$

which may be rewritten as

$$
\eta_{i t}\left(\boldsymbol{\beta}^{*}\right)=f\left(\mu_{i t}\left(\boldsymbol{\beta}^{*}\right)\right)=\mathbf{x}_{i t}^{*^{\prime}} \boldsymbol{\beta}^{*}
$$

where $\mathbf{x}_{i t}^{*}=\left(\mathbf{x}_{i t}^{\prime}, \Phi_{i t}^{\prime}\right)^{\prime}, \boldsymbol{\beta}^{*}=\left(\boldsymbol{\beta}^{\prime}, \boldsymbol{\gamma}^{\prime}\right)^{\prime}$.

\subsubsection{Selection of Number and Location of Knots}

The performance of regression spline (4.8) depends strongly on the selection of best locations of knots. However, the selection of the degree of regression spline basis (4.6) is commonly less crucial and is frequently taken as 1,2 or 3 for computational 
convenience. Three widely used methods for location of knots are given in the book of Wu and Zhang (2006). The methods are briefly discussed below

1) Equally spaced method: This technique takes equally spaced " $q$ " points over the interval $[a, b]$ as knots, where " $q$ " knots are expressed as

$$
\zeta_{r}=a+(b-a) r /(q+1), \quad r=1,2, \ldots, q .
$$

The technique of knots insertion and design time points are independent of one another. It is often used when design-time points are supposed to be scattered uniformly over the range of interest.

2) Equally spaced sample quantiles as knots method: This technique takes uniformly distributed sample quantiles of $z_{i t}$ as knots of the design time points. Let the order statistics be denoted by $z_{(1)}, \ldots, z_{(N)}$ of the design time points. Than $q$ knots are expressed as

$$
\zeta_{r}=z_{(1+[r N /(q+1)])}, \quad r=1,2, \ldots, q,
$$

where $a=[r N /(q+1)]$ expresses the integer portion of $a$. This technique of knots insertion is designed flexibly. When design time points are more dispersed, it traces more knots. It is very similar to the equally spaced method in case of evenly scattered design time points.

3) Model selection based method: This technique takes knots from all the different design time points to get a trimmed basis of power. Knots deletion is equal to the deletion of the trimmed basis of power related to the knots. This technique is similar to the covariates deletion in the approximate linear model. Molinari et al. (2004) note that the selection of knots may be done through the technique of model selection, such as backward elimination or step-wise regression for adding or removing a knot from the nominees of knots. Smith and Kohn (1996), Friedman 
et al. (1991) and Friedman and Silverman (1989) discuss other techniques for the selection of knots. He et al. (2005) mentioned that the regression spline provides good approximations with a small number of knots and, another interesting result is that it is safer to use a small number of knots (near regression splines case), as this results in a smaller mean square error (with optimal rate), whereas this optimal rate cannot be achieved for the other situation that is similar to smoothing splines, without placing strong assumptions on boundary behaviour.

For simplicity, we use the equally spaced sample quantiles of $\left\{z_{i t}, 1 \leq i \leq k, 1 \leq\right.$ $t \leq T\}$ as knots and the number of knots to be chosen as the integer part of $\mathrm{M}^{1 / 5}$, where $\mathrm{M}$ is the number of distinct values in $\left\{z_{i t}, 1 \leq i \leq k, 1 \leq t \leq T\right\}$ (He et al., 2005 and Zhou et al., 2008).

\subsection{Partially Linear Binary Longitudinal Model}

\subsubsection{Logistic Model}

Let $y_{i t}$ be a binary response from subjects $i$ at time $t$. Define $p_{i t}=E\left(y_{i t} \mid \mathbf{X}_{i}, \mathbf{Z}_{i}\right)=$ $P\left(y_{i t}=1 \mid \mathbf{X}_{i}, \mathbf{Z}_{i}\right)$, where the mean of $y_{i t}$ depends only on the covariate vector for subject $i$ at time $t$, so that $p_{i t}=E\left(y_{i t} \mid \mathbf{x}_{i t}, z_{i t}\right)$ (Pepe and Couper, 1997). Consider a partially linear model for $p_{i t}$ as

$$
\operatorname{logit}\left(p_{i t}\right)=\log \left(\frac{p_{i t}}{1-p_{i t}}\right)=\mathbf{x}_{i t}^{*^{\prime}} \boldsymbol{\beta}^{*}
$$

where

$$
\mathbf{x}_{i t}^{*^{\prime}} \boldsymbol{\beta}^{*}=\mathbf{x}_{i t}^{\prime} \boldsymbol{\beta}+\Phi_{i t}^{\prime} \boldsymbol{\gamma}
$$

$\mathbf{x}_{i t}^{*}=\left(\mathbf{x}_{i t}^{\prime}, \quad \Phi_{i t}^{\prime}\right)^{\prime}$ and $\boldsymbol{\beta}^{*}=\left(\boldsymbol{\beta}^{\prime}, \boldsymbol{\gamma}^{\prime}\right)^{\prime}$ is a combined regression parameter vector.

Assume a Bernoulli distribution for the binary response $y_{i t}$, that is

$$
y_{i t} \sim \operatorname{Bernoulli}\left(p_{i t}\right), \quad i=1, \ldots, k, \quad t=1, \ldots, T
$$


with the probability of success

$$
p_{i t}=E\left(y_{i t} \mid \mathbf{X}_{i}, \mathbf{Z}_{i}\right)=P\left(y_{i t}=1 \mid \mathbf{X}_{i}, \mathbf{Z}_{i}\right)
$$

The marginal variance of $y_{i t}$ is stated as a function of the marginal mean:

$$
\operatorname{var}\left(y_{i t} \mid \mathbf{x}_{i t}, z_{i t}\right)=p_{i t}\left(1-p_{i t}\right)
$$

Assume that $y_{i t}$ and $y_{i^{\prime}} t^{\prime}$ are uncorrelated when $i \neq i^{\prime}$. Let

$$
\operatorname{corr}\left(y_{i t}, y_{i t^{\prime}}\right)=\alpha_{t t^{\prime}}
$$

be the correlation between $y_{i t}$ and $y_{i t^{\prime}}$ for given $\mathbf{x}_{i t}$, where $\boldsymbol{\alpha}=\left(\alpha_{12}, \ldots, \alpha_{1 T}, \alpha_{23}\right.$, $\left.\ldots, \alpha_{T-1, T}\right)^{\prime}$ is the vector of correlation parameters.

\subsubsection{Method of Estimation}

\subsubsection{Generalized Estimating Equations}

Our main interest is in estimating the regression parameters $\boldsymbol{\beta}^{*^{\prime}}=\left(\boldsymbol{\beta}^{\prime}, \boldsymbol{\gamma}^{\prime}\right)$ and association parameters $\boldsymbol{\alpha}$. As a naive technique, longitudinal data are analyzed by assuming an identity function for $m($.$) , where the model parameters are estimated$ based on the generalized estimating equations (GEEs) of Liang and Zeger (1986).

The regression parameters $\boldsymbol{\beta}^{*}$ may be estimated by solving the equations

$$
\begin{aligned}
\mathbf{U}_{\boldsymbol{\beta}^{*}}\left(\boldsymbol{\beta}^{*}, \boldsymbol{\alpha}\right) & =\sum_{i=1}^{k} \mathbf{D}_{i}^{\prime} \mathbf{V}_{i}^{-1}\left(\mathbf{y}_{i}-\mathbf{p}_{i}\left(\boldsymbol{\beta}^{*}\right)\right) \\
& =\sum_{i=1}^{k} \mathbf{X}_{i}^{*^{\prime}} \boldsymbol{\Delta}_{i}\left(\mathbf{p}_{i}\left(\boldsymbol{\beta}^{*}\right)\right) \mathbf{V}_{i}^{-1}\left(\mathbf{p}_{i}\left(\boldsymbol{\beta}^{*}, \boldsymbol{\alpha}\right)\right)\left(\mathbf{y}_{i}-\mathbf{p}_{i}\left(\boldsymbol{\beta}^{*}\right)\right)=0
\end{aligned}
$$

where $\mathbf{D}_{i}=\partial \mathbf{p}_{i} / \partial \boldsymbol{\beta}^{*}=\boldsymbol{\Delta}_{i}\left(\mathbf{p}_{i}\left(\boldsymbol{\beta}^{*}\right) \mathbf{X}_{i}^{*}\right.$ with $\mathbf{X}_{i}^{*}=\left(\mathbf{X}_{i}, \boldsymbol{\Phi}_{i}\right), \boldsymbol{\Delta}_{i}\left(\mathbf{p}_{i}\left(\boldsymbol{\beta}^{*}\right)\right)=\partial \mathbf{p}_{i} / \partial \boldsymbol{\eta}_{i}=$ $\operatorname{diag}\left\{\dot{p}_{i 1}\left(\boldsymbol{\beta}^{*}\right), \ldots, \dot{p}_{i T}\left(\boldsymbol{\beta}^{*}\right)\right\}$, with $\dot{p}_{i t}($.$) being the first derivatives of \mathbf{p}($.$) evaluated at$ 
$\boldsymbol{\beta}^{*}, \mathbf{V}_{i}=\left(\mathbf{A}_{i}\right)^{1 / 2} \boldsymbol{\rho}(\boldsymbol{\alpha})\left(\mathbf{A}_{i}\right)^{1 / 2}$, with $\mathbf{A}_{i}=\operatorname{diag}\left\{p_{i 1}\left(1-p_{i 1}\right), p_{i 2}\left(1-p_{i 2}\right) \ldots, p_{i T}(1-\right.$ $\left.\left.p_{i T}\right)\right\}$, and $\boldsymbol{\rho}(\boldsymbol{\alpha})$ is a working correlation matrix for $\mathbf{y}_{i}$, depending on the vector of correlation parameters $\boldsymbol{\alpha}$. For the estimates of $\boldsymbol{\beta}^{*}$, an iterative method is used to solve the equation (4.18). If $\boldsymbol{\alpha}$ were known, then the solution to equation (4.18) is efficient (Liang and Zeger, 1986).

Prentice (1988) extended the GEE approach for the simultaneous estimation of both regression parameters $\boldsymbol{\beta}^{*}$ and correlation parameters $\boldsymbol{\alpha}$. Define

$$
r_{i t u}=r_{i t u}\left(\boldsymbol{\beta}^{*}\right)=\frac{\left(y_{i t}-p_{i t}\right)\left(y_{i u}-p_{i u}\right)}{\left(p_{i t} q_{i t} p_{i u} q_{i u}\right)^{1 / 2}}
$$

which has the mean $\delta_{i t u}$ and variance

$$
\omega_{i t u}=1+\left(1-2 p_{i t}\right)\left(1-2 p_{i u}\right)\left(p_{i t} q_{i t} p_{i u} q_{i u}\right)^{-1 / 2} \delta_{i t u}-\delta_{i t u}^{2},
$$

for $t<u<T, i=1, \ldots, k$, and $t=1, \ldots, T$. Let $\mathbf{r}_{i}=\left(r_{i 12}, \ldots, r_{i 1 T}, r_{i 23}, \ldots, r_{i, T-1, T}\right)^{\prime}$ and $\boldsymbol{\delta}_{i}=\left(\delta_{i 12}, \ldots, \delta_{i 1 T}, \delta_{i 23}, \ldots, \delta_{i, T-1, T}\right)^{\prime}$. Then Prentice (1988) finds the GEE estimators of $\left(\boldsymbol{\beta}^{*}, \boldsymbol{\alpha}\right)$ by solving the estimating equations:

$$
\mathbf{U}_{\boldsymbol{\beta}^{*}}\left(\boldsymbol{\beta}^{*}, \boldsymbol{\alpha}\right)=\sum_{i=1}^{k} \mathbf{D}_{i}^{\prime} \mathbf{V}_{i}^{-1}\left(\mathbf{y}_{i}-\mathbf{p}_{i}\left(\boldsymbol{\beta}^{*}\right)\right)=0
$$

and

$$
\mathbf{U}_{\boldsymbol{\alpha}}\left(\boldsymbol{\beta}^{*}, \boldsymbol{\alpha}\right)=\sum_{i=1}^{k} \mathbf{L}_{i}^{\prime} \mathbf{W}_{i}^{-1}\left(\mathbf{r}_{i}-\boldsymbol{\delta}_{i}\right)=0,
$$

where $\mathbf{L}_{i}=\partial \boldsymbol{\delta}_{i} / \partial \boldsymbol{\alpha}$ and $\mathbf{W}_{i}=\operatorname{diag}\left\{\omega_{i 12}, \ldots, \omega_{i 1 T}, \omega_{i 23}, \ldots, \omega_{i, T-1, T}\right\}$.

\subsubsection{Estimation Algorithm}

Recall the binary response $y_{i t}$ with the probability of success $p_{i t}$ being approximated by

$$
\operatorname{logit}\left(p_{i t}\right)=\log \left(\frac{p_{i t}}{1-p_{i t}}\right)=\mathbf{x}_{i t}^{*^{\prime}} \boldsymbol{\beta}^{*}
$$


where

$$
\mathbf{x}_{i t}^{*^{\prime}} \boldsymbol{\beta}^{*}=\mathbf{x}_{i t}^{\prime} \boldsymbol{\beta}+m\left(z_{i t}\right)=\mathbf{x}_{i t}^{*^{\prime}} \boldsymbol{\beta}+\boldsymbol{\Phi}_{i t}^{\prime} \boldsymbol{\gamma}
$$

Suppose estimators $\hat{\boldsymbol{\beta}}^{*}=\left(\hat{\boldsymbol{\beta}}^{\prime}, \hat{\boldsymbol{\gamma}}^{\prime}\right)^{\prime}$ are obtained by minimizing the objective function

$$
\sum_{i=1}^{k}\left(\mathbf{y}_{i}-\mathbf{p}\left(\mathbf{X}_{i}^{*} \boldsymbol{\beta}^{*}\right)\right)^{\prime} \mathbf{V}_{i}^{-1}\left(\mathbf{y}_{i}-\mathbf{p}\left(\mathbf{X}_{i}^{*} \boldsymbol{\beta}^{*}\right)\right)
$$

or, equivalently, by solving the estimating equations

$$
\mathbf{U}_{\boldsymbol{\beta}^{*}}\left(\boldsymbol{\beta}^{*}, \boldsymbol{\alpha}, m(.)\right)=\sum_{i=1}^{k} \mathbf{D}_{i}^{\prime} \mathbf{V}_{i}^{-1}\left(\mathbf{y}_{i}-\mathbf{p}\left(\mathbf{X}_{i}^{*} \boldsymbol{\beta}^{*}\right)\right)=0
$$

for $\hat{\boldsymbol{\beta}}^{*}$, and

$$
\mathbf{U}_{\boldsymbol{\alpha}}\left(\boldsymbol{\beta}^{*}, \boldsymbol{\alpha}, m(.)\right)=\sum_{i=1}^{k} \mathbf{L}_{i}^{\prime} \mathbf{W}_{i}^{-1}\left(\mathbf{r}_{i}-\boldsymbol{\delta}_{i}\right)=0,
$$

for $\hat{\boldsymbol{\alpha}}$. We describe below an algorithm for simultaneously estimating the mean parameters $\boldsymbol{\beta}^{*}$ and correlation parameters $\boldsymbol{\alpha}$. Here we adopt the regression spline smoother and Prentice (1988) GEE approach for simultaneous estimation of $\left(\boldsymbol{\beta}^{*}, m(),. \boldsymbol{\alpha}\right)$.

Step 0. Consider initial values $\left(\hat{\boldsymbol{\beta}}_{0}^{*}, \hat{\boldsymbol{\alpha}}_{0}\right)$,

Step 1. For a given number and locations of knots, approximate $m\left(z_{i t}\right)$ by $\hat{m}\left(z_{i t}\right)=\sum_{j=0}^{l} \hat{\gamma}_{j o} z_{i t}^{j}+\sum_{r=1}^{q} \hat{\gamma}_{l+r, o}\left(z_{i t}-\zeta_{r}\right)_{+}^{l}$.

Step 2. Given the approximate $\hat{m}(z)$, update $\left(\boldsymbol{\beta}^{*}, m(),. \boldsymbol{\alpha}\right)$ by equations

$$
\mathbf{U}_{\boldsymbol{\beta}^{*}}\left(\boldsymbol{\beta}^{*}, \boldsymbol{\alpha}, m(.)\right)=\sum_{i=1}^{k} \mathbf{D}_{i}^{\prime} \mathbf{V}_{i}^{-1}\left(\mathbf{y}_{i}-\mathbf{p}_{i}\left(\boldsymbol{\beta}^{*}\right)\right)=0
$$

for $\hat{\boldsymbol{\beta}}^{*}$, and

$$
\mathbf{U}_{\boldsymbol{\alpha}}\left(\boldsymbol{\beta}^{*}, \boldsymbol{\alpha}, m(.)\right)=\sum_{i=1}^{k} \mathbf{L}_{i}^{\prime} \mathbf{W}_{i}^{-1}\left(\mathbf{r}_{i}-\boldsymbol{\delta}_{i}\right)=0,
$$


for $\hat{\boldsymbol{\alpha}}$, where $\mathbf{p}_{i}\left(\boldsymbol{\beta}^{*}\right)=\left(p_{i 1}\left(\boldsymbol{\beta}^{*}, \ldots, p_{i T}\left(\boldsymbol{\beta}^{*}\right)\right)^{\prime}\right.$ with

$$
p_{i t}\left(\boldsymbol{\beta}^{*}\right)=\operatorname{logit}^{-1}\left(\mathbf{x}_{i t}^{\prime} \boldsymbol{\beta}+\hat{m}\left(z_{i t}\right)\right)
$$

Step 3. Redo Steps 1 and $\mathbf{2}$ until convergence in $\left(\hat{\boldsymbol{\beta}}^{*}, \hat{\boldsymbol{\alpha}}\right)$.

When executing the above-mentioned algorithm, it is practical to choose an initial value by the GEE method and assuming the identity function for $m($.$) . In$ our experience, the above procedure is not very sensitive to the starting values. The convergence criterion may be based on the comparison between the estimates of two successive reiterations.

\subsubsection{Computational Details for Estimating the Response Model}

Recall the algorithm discussed in Section 4.4.2.2. In Step 1, we approximate the nonlinear function $m($.$) by the spline regression$

$$
\hat{m}\left(z_{i t}\right)=\Phi_{i t}^{\prime} \gamma
$$

for which the success probability $p_{i t}$ is approximated by,

$$
\operatorname{logit}\left(p_{i t}\right)=\mathbf{x}_{i t}^{*^{\prime}} \boldsymbol{\beta}^{*}=\mathbf{x}_{i t}^{\prime} \boldsymbol{\beta}+\Phi_{i t}^{\prime} \boldsymbol{\gamma}
$$

The iterative procedure for $\left(\hat{\boldsymbol{\beta}}^{*}, \hat{\boldsymbol{\alpha}}\right)$ begins with some initial values $\left(\hat{\boldsymbol{\beta}}_{0}^{*}, \hat{\boldsymbol{\alpha}}_{0}\right)$ in step $\mathbf{0}$ of our algorithm and then produces updated values $\left(\hat{\boldsymbol{\beta}}_{s+1}^{*}, \hat{\boldsymbol{\alpha}}_{s+1}\right)$ from $\left(\hat{\boldsymbol{\beta}}_{s}^{*}, \hat{\boldsymbol{\alpha}}_{s}\right)$ by means of the iterative equations

$$
\hat{\boldsymbol{\beta}}_{s+1}^{*}=\hat{\boldsymbol{\beta}}_{s}^{*}+\left(\sum_{i=1}^{k} \mathbf{D}_{i}^{\prime} \mathbf{V}_{i}^{-1} \mathbf{D}_{i}\right)^{-1} \sum_{i=1}^{k} \mathbf{D}_{i}^{\prime} \mathbf{V}_{i}^{-1}\left(\mathbf{y}_{i}-\mathbf{p}_{i}\left(\boldsymbol{\beta}^{*}, \boldsymbol{\alpha}\right)\right),
$$

and

$$
\hat{\boldsymbol{\alpha}}_{s+1}=\hat{\boldsymbol{\alpha}}_{s}+\left(\sum_{i=1}^{k} \mathbf{L}_{i}^{\prime} \mathbf{W}_{i}^{-1} \mathbf{L}_{i}\right)^{-1} \sum_{i=1}^{k} \mathbf{L}_{i}^{\prime} \mathbf{W}_{i}^{-1}\left(\mathbf{r}_{i}-\boldsymbol{\delta}_{i}\right),
$$

for $s=0,1,2, \ldots$, where the second term on the right side of each estimating 
equation is evaluated at the current estimates $\left(\hat{\boldsymbol{\beta}}_{s}^{*}, \hat{\boldsymbol{\alpha}}_{s}\right)$.

\subsubsection{Asymptotic Properties}

\subsubsection{Asymptotic for $\hat{m}($.}

In this section, the asymptotic properties of the proposed estimators are discussed. We first introduce some notation and regularity conditions. Let $\boldsymbol{\beta}_{o}, \boldsymbol{\alpha}_{o}$ and $m_{o}($.$) be the true values of \boldsymbol{\beta}, \boldsymbol{\alpha}$ and $m($.$) , respectively. The estimators \hat{\boldsymbol{\beta}}, \hat{\boldsymbol{\alpha}}$ and $\hat{m}(z)$ are called consistent estimators of $\boldsymbol{\beta}, \boldsymbol{\alpha}$ and $m($.$) if \hat{\boldsymbol{\beta}}-\boldsymbol{\beta}_{o}, \hat{\boldsymbol{\alpha}}-\boldsymbol{\alpha}_{o}$ and $\sup _{z}\left|\hat{m}(z)-m_{o}(z)\right|$ converge to zero in probability. Recall that the mean response model (4.13) is approximated by truncated polynomials. We can develop the asymptotic properties of regression spline estimators using similar arguments as used in De Boor (1978) for B-splines. Both methods are directly linked and comparable, as from truncated basis $\Phi_{l, q}(z)$, we can construct the usual B-spline basis through $B_{l, q}=q^{l} \Phi_{l, q} J_{l, q}$, where $J_{l, q}$ is an invertible dimension matrix of order $(l+q) \times(l+q)$ built from a matrix of $(l+1)^{t h}$ order differences (see, Kauermann et al., 2009 and Claeskens et al., 2009, for more details). The representation on spline can now be written as $B_{l, q} \boldsymbol{\Gamma}=\Phi_{l, q} \gamma$ with coefficient $\boldsymbol{\Gamma}=q^{-l} J_{l, q}^{-1} \gamma$. Suppose $\Pi=\left(B_{1}, \ldots, B_{N_{q}}\right)^{\prime}$ is an $N_{q}=q+l$ vector of B-spline basis functions of order $l$. Let the true mean of $\mathbf{y}_{i}$ be $\mathbf{p}_{0 i}$.

The basic assumptions are as follows:

A1. The distinct design points $\left\{z_{i t}\right\}$ are scattered according to a design density with a compact support on $[0,1]$.

A2. The $h^{\text {th }}$ derivative of $m($.$) is bounded for some h \geq 2$.

A3. There exist a positive constant $C 1$ such that $0<C 1 \leq \nu()<.\infty$, where the functions $\nu(),. f($.$) have bounded second derivatives.$

A4. There exits some $\boldsymbol{\alpha}_{o}$ such that the estimates of covariance parameters satisfy $\sqrt{n}\left(\hat{\boldsymbol{\alpha}}-\boldsymbol{\alpha}_{o}\right)=O_{p}(1)$.

Certain assumptions on covariates of $\mathbf{x}$ and $\mathbf{z}$ are required to assess the asymptotic 
properties of the estimators of $\boldsymbol{\beta}$. To this end, the following relation is assumed:

$$
x_{i t j}=g_{j}\left(z_{i t}\right)+\delta_{i t j}
$$

for $1 \leq i \leq k, 1 \leq t \leq T, 1 \leq j \leq p$, where $g_{j}(z)$ are bounded function $h^{\text {th }}$ derivatives and $\delta_{i t j}$ is an independent random variable with mean zero and is independent of the corresponding random error. The relationship (4.34) is commonly made in the spline literature (see He et al., 2002 and Rice, 1986, among others).

Let $\mathbf{X}=\left(\mathbf{X}_{1}^{\prime}, \ldots, \mathbf{X}_{k}^{\prime}\right)^{\prime}$ with $\mathbf{X}_{i}=\left(\mathbf{x}_{i 1}, \ldots, \mathbf{x}_{i T}\right)^{\prime}, \mathbf{M}=\left(\mathbf{\Pi}_{1}^{\prime}, \ldots, \mathbf{\Pi}_{k}^{\prime}\right)^{\prime}$ with $\boldsymbol{\Pi}_{i}=\left(\Pi_{i 1}, \ldots, \Pi_{i T}\right)^{\prime}, \boldsymbol{\Sigma}=\operatorname{diag}\left(\boldsymbol{\Sigma}_{1}, \ldots, \boldsymbol{\Sigma}_{k}\right)$ with $\boldsymbol{\Sigma}_{i}=\boldsymbol{\Delta}_{i} \mathbf{V}_{i}^{-1} \boldsymbol{\Delta}_{i}$, $\mathbf{P}=\mathbf{M}\left(\mathbf{M}^{\prime} \boldsymbol{\Sigma}_{o} \mathbf{M}\right)^{-\mathbf{1}} \mathbf{M}^{\prime} \boldsymbol{\Sigma}_{o}$, and $\tilde{\mathbf{X}}=\left(\tilde{\mathbf{X}}_{1}^{\prime} \ldots, \tilde{\mathbf{X}}_{k}^{\prime}\right)^{\prime}=(\mathbf{I}-\mathbf{P}) \mathbf{X}$. Also let $\boldsymbol{\Sigma}_{o i}, \tilde{\mathbf{X}}_{o i}$ and $\boldsymbol{\Delta}_{o i}$ represent $\boldsymbol{\Sigma}_{i}, \tilde{\mathbf{X}}_{i}$ and $\boldsymbol{\Delta}_{i}$ evaluated at true $\mathbf{p}_{o i}$. Let $\boldsymbol{\Omega}_{n}$ be an $N(=k \times T) \times p$ matrix whose $s^{\text {th }}$ column is $\boldsymbol{\delta}=\left(\delta_{11 s}, \ldots, \delta_{1 T s}, \ldots, \delta_{k T s}\right)^{\prime}$.

A5. (a) $E\left(\boldsymbol{\Omega}_{n}\right)=0, \sup _{n} \frac{1}{n} E\left(\left\|\boldsymbol{\Omega}_{n}\right\|^{2}\right)<\infty$.

(b) $q \mathbf{M}^{\prime} \boldsymbol{\Sigma}_{o} \mathbf{M}$ is non-singular, and for a sufficiently large $\mathrm{n}$, the eigenvalues of $q \mathbf{M}^{\prime} \boldsymbol{\Sigma}_{o} \mathbf{M} / n$ are bounded, away from 0 and infinity, where $\boldsymbol{\Sigma}_{o}=\operatorname{diag}\left(\boldsymbol{\Sigma}_{o 1}, \ldots, \boldsymbol{\Sigma}_{o k}\right)$. (c) $\frac{1}{n} \mathbf{H}_{n} \rightarrow \mathbf{H}>0, \frac{1}{n} \mathbf{O}_{n} \rightarrow \mathbf{O}>0$ for some positive definite matrices $\mathbf{H}$ and $\mathbf{O}$, where $\mathbf{H}_{n}=\tilde{\mathbf{X}}^{\prime} \boldsymbol{\Sigma}_{o} \tilde{\mathbf{X}}$ and $\left.\mathbf{O}_{n}=\sum_{i=1}^{k} \tilde{\mathbf{X}}_{i}^{\prime} \boldsymbol{\Delta}_{o i} \mathbf{V}_{o i}^{-1} \operatorname{cov}\left(\mathbf{y}_{i}-\mathbf{p}_{o i}\right)\right) \mathbf{V}_{o i}^{-1} \boldsymbol{\Delta}_{o i} \tilde{\mathbf{X}}_{i}$.

Theorem 1. Assume that the above conditions hold. If the number of knots $q \approx n^{1 /(2 h+1)}$, then

$$
\frac{1}{n} \sum_{i=1}^{k} \sum_{t=1}^{T}\left(\hat{m}\left(z_{i t}\right)-m\left(z_{i t}\right)\right)^{2}=O_{p}\left(n^{-2 h /(2 h+1)}\right),
$$

where $\hat{m}(z)=\Pi(z)^{\prime} \hat{\boldsymbol{\Gamma}}$ and

$$
\sqrt{n}\left(\hat{\boldsymbol{\beta}}-\boldsymbol{\beta}_{o}\right) \longrightarrow \mathbf{N}\left(\mathbf{0}, \mathbf{V}_{\boldsymbol{\beta}}\right)
$$

where $\mathbf{V}_{\boldsymbol{\beta}}=\mathbf{H}_{n}^{-1} \mathbf{O}_{n} \mathbf{H}_{n}^{-1}$. The asymptotic covariance matrices of $\hat{\boldsymbol{\beta}}$ can be estimated by

$$
\mathbf{V}(\hat{\boldsymbol{\beta}})=\mathbf{H}_{\hat{\boldsymbol{\beta}}}^{-1} \mathbf{O}_{\hat{\boldsymbol{\beta}}} \mathbf{H}_{\hat{\boldsymbol{\beta}}}^{-1},
$$


where $\mathbf{H}_{\hat{\boldsymbol{\beta}}}=\sum_{i=1}^{k} \widehat{\mathbf{D}}_{i}^{\prime} \hat{\mathbf{V}}_{i}^{-1} \widehat{\mathbf{D}}_{i}$ and $\mathbf{O}_{\hat{\boldsymbol{\beta}}}=\sum_{i=1}^{k} \widehat{\mathbf{D}}_{i}^{\prime} \hat{\mathbf{V}}_{i}^{-1}\left(\mathbf{y}_{i}-\hat{\mathbf{p}}_{i}\right)\left(\mathbf{y}_{i}-\hat{\mathbf{p}}_{i}\right)^{\prime} \hat{\mathbf{V}}_{i}^{-1} \widehat{\mathbf{D}}_{i}$.

For asymptotic consistency, the dimension of the approximating spline space must increase with the number of subjects $k$ and number of knots must be selected appropriately to balance between the bias and variance. Under rather general conditions (see, e.g., Lemma 8 and 9 in Stone, 1985), equation (4.35) implies that $\int\left(\left(\hat{m}\left(z_{i t}\right)-m\left(z_{i t}\right)\right)^{2} d t=O_{p}\left(n^{-2 h /(2 h+1)}\right)\right.$. Under the smoothness condition A2, this is the optimal rate of convergence for estimating $\mathrm{m}($.$) .$

The following Lemma plays an important role in the proof of the theorem and can be found in He et al. (2005) [Lemma 1].

Lemma 1. Under Assumptions A1 and A2, there exist a constant $C_{2}$ such that

$$
\sup _{z \in[0,1]}\left|m(z)-\Pi(z)^{\prime} \boldsymbol{\Gamma}\right| \leq C_{2} q^{-h}
$$

where $\boldsymbol{\Gamma}$ is a vector of $N_{q}$ dimension depending on $\mathrm{m}($.$) . We approximate m(z)$ by $\Pi(z)^{\prime} \boldsymbol{\Gamma}$, thus we have $\eta_{i t}\left(\boldsymbol{\beta}^{*}\right)=f\left(p_{i t}\left(\boldsymbol{\beta}^{*}\right)=\mathbf{x}_{i t}^{\prime} \boldsymbol{\beta}+\Pi_{i t}^{\prime} \boldsymbol{\Gamma}\right.$, with $\boldsymbol{\beta}^{*}=\left(\boldsymbol{\beta}^{\prime}, \boldsymbol{\Gamma}^{\prime}\right)_{\left(p+N_{q}\right) \times 1}^{\prime}$.

\section{Proof of Theorem 1:}

Let

$$
\mathbf{T}_{n}=\left(\begin{array}{cc}
\mathbf{H}_{n}^{-1 / 2} & -\mathbf{H}_{n}^{-1 / 2} \mathbf{X}^{\prime} \boldsymbol{\Sigma}_{\mathbf{o}} \mathbf{M}\left(\mathbf{M}^{\prime} \boldsymbol{\Sigma}_{o} \mathbf{M}\right)^{-\mathbf{1}} \\
0 & q^{-1 / 2} \mathbf{K}_{n}^{-1}
\end{array}\right)
$$

where $\mathbf{H}_{n}=\tilde{\mathbf{X}}^{\prime} \boldsymbol{\Sigma}_{o} \tilde{\mathbf{X}}=\sum_{i=1}^{k} \tilde{\mathbf{X}}_{i}^{\prime} \boldsymbol{\Sigma}_{o i} \tilde{\mathbf{X}}_{i}$ and $\mathbf{K}_{n}^{2}=q \mathbf{M}^{\prime} \boldsymbol{\Sigma}_{o} \mathbf{M}$.

Next, define

$$
\begin{aligned}
\boldsymbol{\xi}\left(\boldsymbol{\beta}^{*}\right) & =\left(\begin{array}{l}
\boldsymbol{\xi}_{\mathbf{1}} \\
\boldsymbol{\xi}_{\mathbf{2}}
\end{array}\right)=\left(\mathbf{T}_{n}^{\prime}\right)^{-1}\left(\boldsymbol{\beta}^{*}-\boldsymbol{\beta}_{o}^{*}\right) \\
& =\left(\begin{array}{c}
\mathbf{H}_{n}^{1 / 2}\left(\boldsymbol{\beta}-\boldsymbol{\beta}_{o}\right) \\
q^{-1 / 2} \mathbf{K}_{n}\left(\boldsymbol{\Gamma}-\boldsymbol{\Gamma}_{o}\right)+q^{1 / 2} \mathbf{K}_{n}^{-1} \mathbf{M}^{\prime} \Sigma_{o} \mathbf{X}\left(\boldsymbol{\beta}-\boldsymbol{\beta}_{o}\right)
\end{array}\right)
\end{aligned}
$$


and

$$
\hat{\xi}=\left(\begin{array}{c}
\hat{\xi_{1}} \\
\hat{\xi_{2}}
\end{array}\right)=\xi(\hat{\beta}, \hat{\Gamma})
$$

From Lemma 1, there exists a constant $C_{o}$ such that for sufficiently large n,

$$
\frac{1}{n} \sum_{i=1}^{k} \sum_{t=1}^{T}\left(\hat{m}\left(z_{i t}\right)-m\left(z_{i t}\right)\right)^{2} \leq \frac{2}{n} \sum_{i=1}^{k} \sum_{t=1}^{T} \Pi\left(z_{i t}\right)^{\prime}\left(\hat{\boldsymbol{\Gamma}}-\boldsymbol{\Gamma}_{\boldsymbol{o}}\right)^{2}+2 C_{o} q^{-2 h}
$$

and $\left\|\mathbf{H}_{n}^{-1 / 2}\left(\hat{\boldsymbol{\beta}}-\boldsymbol{\beta}_{o}\right)\right\| \leq\|\hat{\boldsymbol{\xi}}\|$. We have

$$
\begin{gathered}
{\left[\frac{1}{n} \sum_{i=1}^{k} \sum_{t=1}^{T} \Pi\left(z_{i t}\right)^{\prime}\left(\hat{\boldsymbol{\Gamma}}-\boldsymbol{\Gamma}_{\boldsymbol{o}}\right)^{2}\right]^{1 / 2}=n^{-1 / 2}\left\|\mathbf{M}\left(\hat{\boldsymbol{\Gamma}}-\boldsymbol{\Gamma}_{\mathbf{o}}\right)\right\| \leq C n^{-1 / 2}\left\|q^{-1 / 2} \mathbf{K}_{n}\left(\hat{\boldsymbol{\Gamma}}-\boldsymbol{\Gamma}_{\boldsymbol{o}}\right)\right\|} \\
\leq C n^{-1 / 2}\|\boldsymbol{\xi}\|+C B_{n}^{-1 / 2}\left\|\left(\hat{\boldsymbol{\beta}}-\boldsymbol{\beta}_{o}\right)\right\| \sup _{\|\mathbf{a}\|=1\|\mathbf{b}\|=1}\left|n^{-1} \mathbf{a}^{\prime} \mathbf{M}^{\prime} \boldsymbol{\Sigma}_{o} \mathbf{X} \mathbf{b} q^{1 / 2}\right|
\end{gathered}
$$

where $B_{n}$ is the minimum eigen value of $q \mathbf{M}^{\prime} \Sigma_{o} \mathbf{M} / n$. Then it is sufficient to show that $\|\hat{\boldsymbol{\xi}}\|=O_{p}\left(q^{1 / 2}\right)$. To do so, we standardize $\boldsymbol{\varrho}_{n i}=\boldsymbol{\Pi}\left(\mathbf{Z}_{i}\right) \boldsymbol{\Gamma}_{\boldsymbol{o}}-m\left(\mathbf{Z}_{i}\right)$, and $\boldsymbol{\varsigma}_{i}=$ $\overline{\mathbf{X}}_{i} \boldsymbol{\xi}_{\mathbf{1}}+\tilde{\boldsymbol{\Pi}}_{i} \boldsymbol{\xi}_{\mathbf{2}}+\boldsymbol{\varrho}_{n i}$, where $\widehat{\mathbf{X}}_{i}=\left(\overline{\mathbf{X}}_{i} \tilde{\boldsymbol{\Pi}}_{i}\right), \overline{\mathbf{X}}_{i}^{\prime}=\mathbf{H}_{n}^{-1 / 2} \tilde{\mathbf{X}}_{i}^{\prime}$ and $\tilde{\boldsymbol{\Pi}}_{i}^{\prime}=q^{1 / 2} \mathbf{K}_{n}^{-1} \boldsymbol{\Pi}\left(\mathbf{Z}_{i}^{\prime}\right)$. Thus the regression problem $f\left(\mathbf{p}_{i}\left(\boldsymbol{\beta}^{*}\right)\right)=\boldsymbol{\eta}_{i}\left(\boldsymbol{\beta}^{*}\right)=\mathbf{X}_{i}^{*} \boldsymbol{\beta}^{*}$ becomes $\boldsymbol{\eta}_{i}(\boldsymbol{\xi})=\mathbf{X}_{i}^{*} \boldsymbol{\beta}^{*}=$ $\boldsymbol{\eta}_{o i}+\boldsymbol{\varsigma}_{i}$, for $i=1, \ldots, k$, where $\boldsymbol{\eta}_{o i}=\mathbf{X}_{i} \boldsymbol{\beta}_{o}+m\left(\mathbf{Z}_{i}\right)$,

$\mathbf{p}_{i}\left(\boldsymbol{\beta}^{*}\right)=f^{-1}\left(\boldsymbol{\eta}_{o i}+\boldsymbol{\varsigma}_{i}\right)$ with $f=$ logit link function. The estimating equation $\mathbf{U}_{\boldsymbol{\beta}^{*}}\left(\mathbf{p}\left(\mathbf{X}^{*} \boldsymbol{\beta}^{*}\right)\right)$ becomes

$$
\mathbf{U}_{\boldsymbol{\xi}}\left(\mathbf{p}\left(\boldsymbol{\eta}_{o i}+\boldsymbol{\varsigma}_{i}\right)\right)=\sum_{i=1}^{k} \mathbf{D}_{i}^{\prime}\left(\mathbf{p}_{i}\left(\boldsymbol{\eta}_{o i}+\boldsymbol{\varsigma}_{i}\right)\right) \mathbf{V}_{i}^{-1}\left(\mathbf{p}_{i}\left(\boldsymbol{\eta}_{o i}+\boldsymbol{\varsigma}_{i}\right)\right)\left(\mathbf{y}_{i}-\mathbf{p}_{i}\left(\boldsymbol{\eta}_{o i}+\boldsymbol{\varsigma}_{i}\right)\right)=0,
$$


where $\mathbf{D}_{i}\left(\boldsymbol{\eta}_{o i}+\boldsymbol{\varsigma}_{i}\right)=\boldsymbol{\Delta}_{i}\left(\boldsymbol{\eta}_{o i}+\boldsymbol{\varsigma}_{i}\right) \mathbf{X}_{i}^{*}$. Thus we have

$$
\begin{aligned}
\boldsymbol{\psi}(\boldsymbol{\xi}) & =\left(\begin{array}{c}
\boldsymbol{\psi}_{\mathbf{1}}(\boldsymbol{\xi}) \\
\boldsymbol{\psi}_{\mathbf{2}}(\boldsymbol{\xi})
\end{array}\right)=\mathbf{T}_{\boldsymbol{n}} \mathbf{U}_{\boldsymbol{\xi}}\left(\mathbf{p}\left(\boldsymbol{\eta}_{o i}+\boldsymbol{\varsigma}_{i}\right)\right) \\
& =\sum_{i=1}^{k} \widehat{\mathbf{D}}_{i}^{\prime}\left(\mathbf{p}_{i}\left(\boldsymbol{\eta}_{o i}+\boldsymbol{\varsigma}_{i}\right)\right) \mathbf{V}_{i}^{-1}\left(\mathbf{p}_{i}\left(\boldsymbol{\eta}_{o i}+\boldsymbol{\varsigma}_{i}\right)\right)\left(\mathbf{y}_{i}-\mathbf{p}_{i}\left(\boldsymbol{\eta}_{o i}+\boldsymbol{\varsigma}_{i}\right)\right) \\
& =\left(\begin{array}{c}
\sum_{i=1}^{k} \mathbf{H}_{n}^{-1 / 2} \tilde{\mathbf{X}}_{i}^{\prime} \boldsymbol{\Delta}_{i}\left(\mathbf { p } _ { i } ( \boldsymbol { \eta } _ { o i } + \boldsymbol { \varsigma } _ { i } ) \mathbf { V } ^ { - 1 } ( \mathbf { p } _ { i } ( \boldsymbol { \eta } _ { o i } + \boldsymbol { \varsigma } _ { i } ) ) \left(\mathbf{y}_{i}-\left(\mathbf{p}_{i}\left(\boldsymbol{\eta}_{o i}+\boldsymbol{\varsigma}_{i}\right)\right)\right.\right. \\
\sum_{i=1}^{k} q^{1 / 2} \mathbf{K}_{n}^{-1} \boldsymbol{\Pi}_{i}^{\prime} \boldsymbol{\Delta}_{i}\left(\mathbf{p}_{i}\left(\boldsymbol{\eta}_{o i}+\boldsymbol{\varsigma}_{i}\right)\right) \mathbf{V}^{-1}\left(\mathbf{p}_{i}\left(\boldsymbol{\eta}_{o i}+\boldsymbol{\varsigma}_{i}\right)\right)\left(\mathbf{y}_{i}-\left(\mathbf{p}_{i}\left(\boldsymbol{\eta}_{o i}+\boldsymbol{\varsigma}_{i}\right)\right)\right.
\end{array}\right)=0
\end{aligned}
$$

where $\widehat{\mathbf{D}}_{i}=\boldsymbol{\Delta}_{i} \widehat{\mathbf{X}}_{i}=\left(\boldsymbol{\Delta}_{i} \overline{\mathbf{X}}_{i}, \quad \boldsymbol{\Delta}_{i} \tilde{\boldsymbol{\Pi}}_{i}\right)=\left(\boldsymbol{\Delta}_{i} \tilde{\mathbf{X}}_{i} \mathbf{H}_{n}^{-1 / 2}, \quad \boldsymbol{\Delta}_{i} \boldsymbol{\Pi}_{i} \mathbf{K}_{n}^{-1} q^{1 / 2}\right)$. It is easy to show that both equations (4.38) and (4.39) give the same root for $\boldsymbol{\xi}$ by $\mathbf{A 4}$. Let $\mathbf{a} \in \mathbf{R}^{p+N_{q}}$, where $\mathbf{a}^{\prime} \mathbf{a}=\mathbf{1}$. We expand $\mathbf{a}^{\prime} \boldsymbol{\psi}(\boldsymbol{\xi})$ in a Taylor series as

$$
\begin{aligned}
\mathbf{a}^{\prime} \boldsymbol{\psi}(\boldsymbol{p}(\boldsymbol{\xi})) & =\mathbf{a}^{\prime} \boldsymbol{\psi}\left(\mathbf{p}\left(\boldsymbol{\eta}_{o}+\boldsymbol{\varsigma}\right)\right) \\
& =\sum_{i=1}^{k} \mathbf{a}^{\prime} \widehat{\mathbf{D}}_{i}^{\prime}\left(\mathbf{p}_{i}\left(\boldsymbol{\eta}_{o i}+\boldsymbol{\varsigma}_{i}\right)\right) \mathbf{V}_{i}^{-1}\left(\mathbf{p}_{i}\left(\boldsymbol{\eta}_{o i}+\boldsymbol{\varsigma}_{i}\right)\right)\left(\mathbf{y}_{i}-\mathbf{p}_{i}\left(\boldsymbol{\eta}_{o i}+\boldsymbol{\varsigma}_{i}\right)\right) \\
& =\sum_{i=1}^{k} \mathbf{a}^{\prime} \widehat{\mathbf{D}}_{o i}^{\prime} \mathbf{V}_{o i}^{-1}\left(\mathbf{y}_{i}-\mathbf{p}_{o i}\right)-\sum_{i=1}^{k} \mathbf{a}^{\prime} \widehat{\mathbf{D}}_{o i}^{\prime} \mathbf{V}_{o i}^{-1} \boldsymbol{\Delta}_{o i} \boldsymbol{\varsigma}_{i} \\
& +\left.\sum_{i=1}^{k} \boldsymbol{\varsigma}_{i}^{\prime} \frac{\boldsymbol{\partial}}{\boldsymbol{\partial} \boldsymbol{\varsigma}_{i}}\left(\mathbf{a}^{\prime} \widehat{\mathbf{D}}_{i}^{\prime}\right)\right|_{\boldsymbol{\varsigma}_{i}=0} \mathbf{V}_{o i}^{-1}\left(\mathbf{y}_{i}-\mathbf{p}_{o i}\right)+\varrho_{n}^{*}\left(\boldsymbol{\varsigma}^{*}\right)
\end{aligned}
$$

where $\varrho_{n}^{*}\left(\varsigma^{*}\right)=\sum_{i=1}^{k} \varrho_{n}^{*}\left(\varsigma_{i}^{*}\right)$ with

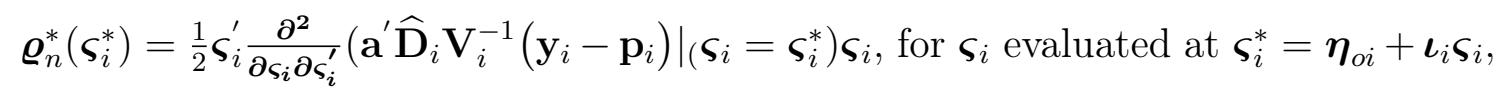
$1 \leq i \leq k, 0<\boldsymbol{\iota}_{i}<1$. Further, we denote

$$
\boldsymbol{\Psi}(\boldsymbol{\xi})=\sum_{i=1}^{k} \widehat{\mathbf{D}}_{o i}^{\prime} \mathbf{V}_{o i}^{-1}\left(\mathbf{y}_{i}-\mathbf{p}_{o i}\right)-\boldsymbol{\xi}=\sum_{i=1}^{k} \widehat{\mathbf{D}}_{o i}^{\prime} \mathbf{V}_{o i}^{-1}\left(\mathbf{y}_{i}-\mathbf{p}_{o i}\right)-\left(\begin{array}{l}
\boldsymbol{\xi}_{\mathbf{1}} \\
\boldsymbol{\xi}_{\mathbf{2}}
\end{array}\right)
$$


The root obtained by solving $\boldsymbol{\Psi}(\boldsymbol{\xi})=0$

$$
\begin{aligned}
\tilde{\boldsymbol{\xi}} & =\left(\begin{array}{c}
\tilde{\boldsymbol{\xi}}_{\mathbf{1}} \\
\tilde{\boldsymbol{\xi}}_{\mathbf{2}}
\end{array}\right)=\sum_{i=1}^{k} \widehat{\mathbf{D}}_{o i}^{\prime} \mathbf{V}_{o i}^{-1}\left(\mathbf{y}_{i}-\mathbf{p}_{o i}\right) \\
& =\left(\begin{array}{c}
\sum_{i=1}^{k} \mathbf{H}_{n}^{-1 / 2} \tilde{\mathbf{X}}_{i}^{\prime} \boldsymbol{\Delta}_{o i} \mathbf{V}_{o i}^{-1}\left(\mathbf{y}_{i}-\mathbf{p}_{o i}\right) \\
\sum_{i=1}^{k} q^{1 / 2} \mathbf{K}_{n}^{-1} \boldsymbol{\Pi}_{i}^{\prime} \boldsymbol{\Delta}_{o i} \mathbf{V}_{o i}^{-1}\left(\mathbf{y}_{i}-\mathbf{p}_{o i}\right)
\end{array}\right)
\end{aligned}
$$

is not an estimator. Below we show that the difference between $\tilde{\xi}$ and $\hat{\boldsymbol{\xi}}$ is small.

The difference between $\mathbf{a}^{\prime} \boldsymbol{\psi}(\boldsymbol{\xi})$ and $\mathbf{a}^{\prime} \boldsymbol{\Psi}(\boldsymbol{\xi})$ can be expressed as

$$
\begin{aligned}
\mathbf{a}^{\prime}(\boldsymbol{\psi}(\boldsymbol{\xi})-\boldsymbol{\Psi}(\boldsymbol{\xi}))= & -\sum_{i=1}^{k} \mathbf{a}^{\prime} \widehat{\mathbf{D}}_{o i}^{\prime} \mathbf{V}_{o i}^{-1} \widehat{\mathbf{D}}_{o i}^{\prime} \boldsymbol{\xi}-\sum_{i=1}^{k} \mathbf{a}^{\prime} \widehat{\mathbf{D}}_{o i}^{\prime} \mathbf{V}_{o i}^{-1} \boldsymbol{\Delta}_{o i} \boldsymbol{\varrho}_{n i} \\
& +\left.\sum_{i=1}^{k} \boldsymbol{\varsigma}_{i}^{\prime} \frac{\boldsymbol{\partial}}{\partial \boldsymbol{\varsigma}_{i}}\left(\mathbf{a}^{\prime} \widehat{\mathbf{D}}_{i}^{\prime}\right)\right|_{\boldsymbol{\varsigma}_{i}=0} \mathbf{V}_{o i}^{-1}\left(\mathbf{y}_{i}-\mathbf{p}_{o i}\right)+\boldsymbol{\varrho}_{n}^{*}\left(\varsigma^{*}\right)+\mathbf{a}^{\prime} \boldsymbol{\xi}
\end{aligned}
$$

since $\boldsymbol{\varsigma}_{i}=\widehat{\mathbf{X}}_{i} \boldsymbol{\xi}+\varrho_{n i}$ and $\widehat{\mathbf{D}}_{o i}=\boldsymbol{\Delta}_{o i} \widehat{\mathbf{X}}_{i}$.

Now, we can show that

$$
\begin{aligned}
\sum_{i=1}^{k} \widehat{\mathbf{D}}_{o i}^{\prime} \mathbf{V}_{o i}^{-1} \widehat{\mathbf{D}}_{o i}^{\prime} & =\sum_{i=1}^{k} \widehat{\mathbf{X}}_{i}^{\prime} \boldsymbol{\Delta}_{o i} \mathbf{V}_{o i}^{-1} \boldsymbol{\Delta}_{o i} \widehat{\mathbf{X}}_{i}=\sum_{i=1}^{k} \widehat{\mathbf{X}}_{i}^{\prime} \boldsymbol{\Sigma}_{o i} \widehat{\mathbf{X}}_{i}=\widehat{\mathbf{X}}^{\prime} \boldsymbol{\Sigma}_{o} \widehat{\mathbf{X}} \\
& =\left(\widehat{\mathbf{X}} \mathbf{H}_{n}^{-1 / 2} \mathbf{M} \mathbf{K}_{n}^{-1} q^{1 / 2}\right)^{\prime} \boldsymbol{\Sigma}_{\boldsymbol{o}}\left(\widehat{\mathbf{X}} \mathbf{H}_{n}^{-1 / 2} \mathbf{M} \mathbf{K}_{\mathbf{n}}^{-\mathbf{1}} \mathbf{q}^{\mathbf{1} / \mathbf{2}}\right) \\
& =\left[\begin{array}{cc}
\mathbf{H}_{n}^{-1 / 2} \tilde{\mathbf{X}}^{\prime} \boldsymbol{\Sigma}_{\mathbf{o}} \tilde{\mathbf{X}} \mathbf{H}_{n}^{-1 / 2} & \mathbf{H}_{n}^{-1 / 2} \tilde{\mathbf{X}}^{\prime} \boldsymbol{\Sigma}_{\mathbf{o}} \mathbf{M} \mathbf{K}_{n}^{-1} q^{1 / 2} \\
q^{1 / 2} \mathbf{K}^{-1} \mathbf{M}^{\prime} \boldsymbol{\Sigma}_{\mathbf{o}} \tilde{\mathbf{X}} \mathbf{H}_{n}^{-1 / 2} & q^{1 / 2} \mathbf{K}_{n}^{-1} \mathbf{M}^{\prime} \boldsymbol{\Sigma}_{\mathbf{o}} \mathbf{M} \mathbf{K}_{n}^{-1} q^{1 / 2}
\end{array}\right] \\
& =\left[\begin{array}{cc}
\mathbf{H}_{n}^{-1 / 2} \mathbf{H}_{n} \mathbf{H}_{n}^{-1 / 2} & \mathbf{H}_{n}^{-1 / 2} \mathbf{X}^{\prime}(\mathbf{I}-\mathbf{P})^{\prime} \boldsymbol{\Sigma}_{\mathbf{o}} \mathbf{M} \mathbf{K}_{n}^{-1} q^{1 / 2} \\
q^{1 / 2} \mathbf{K}^{-1} \mathbf{M}^{\prime} \boldsymbol{\Sigma}_{\mathbf{o}} \mathbf{X}(\mathbf{I}-\mathbf{P}) \mathbf{H}_{n}^{-1 / 2} & \mathbf{K}_{n}^{-1} q^{-1} \mathbf{K}_{n}^{2} \mathbf{K}_{n}^{-1} q^{1 / 2}
\end{array}\right] \\
& =\left[\begin{array}{cc}
\mathbf{I}_{p} & \mathbf{0} \\
\mathbf{0} & \mathbf{I}_{N_{q}}
\end{array}\right]=\mathbf{I}_{\left(p \times N_{q}\right) \times\left(p \times N_{q}\right),}
\end{aligned}
$$

since

$\mathbf{H}_{n}^{-1 / 2} \mathbf{X}^{\prime}(\mathbf{I}-\mathbf{P})^{\prime} \boldsymbol{\Sigma}_{\mathbf{o}} \mathbf{M} \mathbf{K}_{n}^{-1} q^{1 / 2}=\mathbf{H}_{n}^{-1 / 2} \mathbf{X}^{\prime} \boldsymbol{\Sigma}_{\mathbf{o}} \mathbf{M K}_{n}^{-1} q^{1 / 2}-\mathbf{H}_{n}^{-1 / 2} \mathbf{X}^{\prime} \boldsymbol{\Sigma}_{\mathbf{o}} \mathbf{M} \mathbf{K}_{n}^{-1} q^{1 / 2}=\mathbf{0}$ 
Thus Eq.(4.42) becomes

$$
\begin{aligned}
\mathbf{a}^{\prime}(\boldsymbol{\psi}(\boldsymbol{\xi})-\boldsymbol{\Psi}(\boldsymbol{\xi})) & =-\sum_{i=1}^{k} \mathbf{a}^{\prime} \widehat{\mathbf{D}}_{o i}^{\prime} \mathbf{V}_{o i}^{-1} \boldsymbol{\Delta}_{o i} \boldsymbol{\varrho}_{n i}+\left.\sum_{i=1}^{k} \boldsymbol{\varsigma}_{i}^{\prime} \frac{\partial}{\partial \boldsymbol{\varsigma}_{i}}\left(\mathbf{a}^{\prime} \widehat{\mathbf{D}}_{i}^{\prime}\right)\right|_{\varsigma_{i}=0} \mathbf{V}_{o i}^{-1}\left(\mathbf{y}_{i}-\mathbf{p}_{o i}\right)+\boldsymbol{\varrho}_{n}^{*}\left(\varsigma^{*}\right) \\
& =: \mathbf{l}_{\mathbf{1}}+\mathbf{l}_{\mathbf{2}}(\boldsymbol{\xi})+\mathbf{l}_{\mathbf{3}}
\end{aligned}
$$

We can show that

$$
\begin{aligned}
\left|\mathbf{l}_{\mathbf{1}}\right| & =\left|\sum_{i=1}^{k} \mathbf{a}^{\prime} \widehat{\mathbf{D}}_{o i}^{\prime} \mathbf{V}_{o i}^{-1} \boldsymbol{\Delta}_{o i} \boldsymbol{\varrho}_{n i}\right|=\left|\sum_{i=1}^{k} \mathbf{a}^{\prime} \widehat{\mathbf{X}}_{i}^{\prime} \boldsymbol{\Delta}_{o i} \mathbf{V}_{o i}^{-1} \boldsymbol{\Delta}_{o i} \boldsymbol{\varrho}_{n i}\right| \\
& =\left|\sum_{i=1}^{k} \mathbf{a}^{\prime} \widehat{\mathbf{X}}_{i}^{\prime} \boldsymbol{\Sigma}_{o i} \boldsymbol{\varrho}_{n i}\right|=\left|\mathbf{a}^{\prime} \widehat{\mathbf{X}}^{\prime} \boldsymbol{\Sigma}_{o} \boldsymbol{\varrho}_{n}\right| \\
& \leq\left\{\mathbf{a}^{\prime} \widehat{\mathbf{X}}^{\prime} \boldsymbol{\Sigma}_{o} \widehat{\mathbf{X}} \mathbf{a}\right\}^{1 / 2}\left\{\boldsymbol{\varrho}_{n}^{\prime} \boldsymbol{\Sigma}_{o} \boldsymbol{\varrho}_{n}\right\}^{1 / 2}=O\left(n^{1 / 2} q^{-h}\right)=O\left(q^{1 / 2}\right),
\end{aligned}
$$

where $\boldsymbol{\varrho}_{n}=\left(\boldsymbol{\varrho}_{n 11}, \ldots, \varrho_{n 1 T}, \ldots, \varrho_{n k T}\right)^{\prime}$ and $q \approx n^{1 /(2 h+1)}$. For $\mathbf{l}_{\mathbf{2}}(\boldsymbol{\xi})$,

let $\mathbf{G}_{o i}=\left.\frac{\partial}{\partial \varsigma_{i}}\left(\mathbf{a}^{\prime} \widehat{\mathbf{D}}_{i}^{\prime}\right)\right|_{\boldsymbol{s}_{i}=0} \mathbf{V}_{o i}^{-1}\left(\mathbf{y}_{i}-\mathbf{p}_{o i}\right)$ be the derivative of $\mathbf{a}^{\prime} \widehat{\mathbf{D}}_{i}^{\prime} \mathbf{V}_{i}^{-1}\left(\mathbf{y}_{i}-\mathbf{p}_{i}\right)$ with respect to $\boldsymbol{\varsigma}_{i}$, evaluated at $\boldsymbol{\varsigma}_{i}=0$. Thus we can write

$$
\begin{aligned}
\mathbf{l}_{\mathbf{2}}(\xi) & =\sum_{i=1}^{k} \boldsymbol{\xi}^{\prime} \widehat{\mathbf{X}}_{i}^{\prime} \mathbf{G}_{o i}+\sum_{i=1}^{k} \varrho_{n i}^{\prime} \mathbf{G}_{o i} \\
& =: \mathbf{l}_{\mathbf{2}}^{\mathbf{1}}(\boldsymbol{\xi})+\mathbf{l}_{\mathbf{2}}^{\mathbf{2}}
\end{aligned}
$$

Let $\tilde{\mathbf{y}}_{i}=\mathbf{V}_{o i}^{-1}\left(\mathbf{y}_{i}-\mathbf{p}_{o i}\right)=\left(\tilde{y}_{i 1}, \ldots, \tilde{y}_{i T}\right)^{\prime}$. It is easy to show that $\mathbf{G}_{o i}=\operatorname{diag}\left(\dot{p}_{o i 1} \tilde{y}_{i 1}, \ldots, \dot{p}_{o i T} \tilde{y}_{i T}\right) \widehat{\mathbf{X}}_{i} \mathbf{a}=: \nabla\left(\tilde{\mathbf{y}}_{i}\right) \widehat{\mathbf{X}}_{i} \mathbf{a}$. Then by Cauchy-Schwarz inequal- 
ity, we have

$$
\begin{aligned}
\left(\mathbf{l}_{\mathbf{2}}^{\mathbf{1}}(\boldsymbol{\xi})\right)^{\mathbf{2}} & =\left(\sum_{i=1}^{k} \boldsymbol{\xi}^{\prime} \widehat{\mathbf{X}}_{i}^{\prime} \nabla\left(\tilde{\mathbf{y}}_{i}\right) \widehat{\mathbf{X}}_{i} \mathbf{a}\right)^{\mathbf{2}} \\
& =\left(\sum_{j=1}^{\bar{p}} \boldsymbol{\xi}_{j} \sum_{i=1}^{k} \mathbf{1}_{j}^{\prime} \widehat{\mathbf{X}}_{i}^{\prime} \nabla\left(\tilde{\mathbf{y}}_{i}\right) \widehat{\mathbf{X}}_{i} \mathbf{a}\right)^{\mathbf{2}} \\
& \leq\|\boldsymbol{\xi}\|^{2} \sum_{j=1}^{\bar{p}}\left(\sum_{i=1}^{k} \mathbf{1}_{j}^{\prime} \widehat{\mathbf{X}}_{i}^{\prime} \nabla\left(\tilde{\mathbf{y}}_{i}\right) \widehat{\mathbf{X}}_{i} \mathbf{a}\right)^{\mathbf{2}} \\
& \leq\|\boldsymbol{\xi}\|^{2} \sum_{j, t=1}^{\bar{p}}\left(\sum_{i=1}^{k} \mathbf{1}_{j}^{\prime} \widehat{\mathbf{X}}_{i}^{\prime} \nabla\left(\tilde{\mathbf{y}}_{i}\right) \widehat{\mathbf{X}}_{i} \mathbf{1}_{t}\right)^{\mathbf{2}}
\end{aligned}
$$

where $\bar{p}=p+N_{q}$ and $\mathbf{1}_{j}=(0, \ldots, 0,1,0, \ldots, 0)^{\prime}$ is a $\bar{p}$ vector with 1 as its $j^{\text {th }}$ element and 0 elsewhere. By assumptions A1, A3, and A5, we have

$$
\begin{aligned}
\mathbf{E}\left(\mathbf{l}_{\mathbf{2}}^{\mathbf{1}}(\boldsymbol{\xi})\right)^{\mathbf{2}} & \leq\|\boldsymbol{\xi}\|^{2} \sum_{j, t=1}^{\bar{p}} \mathbf{E}\left(\sum_{i=1}^{k} \mathbf{1}_{j}^{\prime} \widehat{\mathbf{X}}_{i}^{\prime} \nabla\left(\tilde{\mathbf{y}}_{i}\right) \widehat{\mathbf{X}}_{i} \mathbf{1}_{t}\right)^{\mathbf{2}} \\
& \leq C\|\boldsymbol{\xi}\|^{2} \sum_{j, t=1}^{\bar{p}} \mathbf{E}\left(\sum_{i=1}^{k} \mathbf{1}_{j}^{\prime} \widehat{\mathbf{X}}_{i}^{\prime} \nabla\left(\tilde{\mathbf{y}}_{i}\right) \widehat{\mathbf{X}}_{i} \mathbf{1}_{t}\right)^{\mathbf{2}} \\
& \leq C\|\boldsymbol{\xi}\|^{2} \sum_{j, t=1}^{\bar{p}} \sum_{i=1}^{k} \mathbf{1}_{j}^{\prime} \widehat{\mathbf{X}}_{i}^{\prime} \widehat{\mathbf{X}}_{i} \mathbf{1}_{j} \mathbf{E}\left\|\nabla\left(\tilde{\mathbf{y}}_{i}\right) \widehat{\mathbf{X}}_{i} \mathbf{1}_{t}\right\|^{2} \\
& \leq C\|\boldsymbol{\xi}\|^{2} \sup _{i} \sum_{j=1} \mathbf{1}_{j}^{\prime} \widehat{\mathbf{X}}_{i}^{\prime} \widehat{\mathbf{X}}_{i} \mathbf{1}_{j} \sum_{i=1}^{k} \sum_{j=1}^{\bar{p}} \mathbf{1}_{j}^{\prime} \widehat{\mathbf{X}}_{i}^{\prime} \widehat{\mathbf{X}}_{i} \mathbf{1}_{j} O(q) \\
& =C\|\boldsymbol{\xi}\|^{2} \sup _{i} \operatorname{trace}\left\{\widehat{\mathbf{X}}_{i} \widehat{\mathbf{X}}_{i}^{\prime}\right\} \operatorname{trace}\left\{\sum_{i=1}^{k} \widehat{\mathbf{X}}_{i} \widehat{\mathbf{X}}_{i}^{\prime}\right\} O(q) \\
& \leq C q\|\boldsymbol{\xi}\|^{\mathbf{2}} \sup _{i} \operatorname{trace}\left\{\tilde{\mathbf{X}}_{i} \mathbf{H}_{n}^{1 / 2} \tilde{\mathbf{X}}_{i}^{\prime}+q^{1 / 2} \boldsymbol{\Pi}_{i} \mathbf{K}_{n}^{-1} \boldsymbol{\Pi}_{i}^{\prime}\right\} O(q) \\
& =O\left(q^{3}\|\boldsymbol{\xi}\|^{\mathbf{2} / n)},\right.
\end{aligned}
$$

where the constant $C$ is independent of $n$ and can differ between two lines. Therefore, we have for enough large $L$,

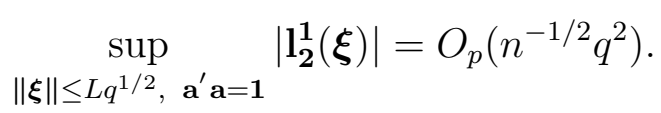


Similarly, we can show that

$$
\sup _{\mathbf{a}^{\prime} \mathbf{a}=\mathbf{1}}\left|\mathbf{l}_{\mathbf{2}}^{\mathbf{2}}\right|=O_{p}\left(q^{1-h}\right)
$$

Combining these two findings together and noting that $q n^{1 /(2 h+1)}$, we have

$$
\sup _{\mathbf{a}^{\prime} \mathbf{a}=\mathbf{1}}\left|\mathbf{l}_{\mathbf{2}}\right|=O_{p}\left(q^{1 / 2}\right) \text {. }
$$

Next, for $\mathbf{l}_{\mathbf{3}}=\boldsymbol{\varrho}_{n}^{*}\left(\varsigma^{*}\right)$, let $\mathbf{F}_{i}^{*}=\frac{\partial^{2}}{\partial \varsigma_{i} \partial \varsigma_{i}^{\prime}}\left(\left.\mathbf{a}^{\prime} \widehat{\mathbf{D}}_{i} \mathbf{V}_{i}^{-1}\left(\mathbf{y}_{i}-\mathbf{p}_{i}\right)\right|_{\left(\boldsymbol{\varsigma}_{i}=\varsigma_{i}^{*}\right)}\right.$. We can show

$$
\begin{aligned}
\mathbf{l}_{\mathbf{3}} & =\frac{1}{2} \sum_{i=1}^{k} \boldsymbol{\xi}^{\prime} \widehat{\mathbf{X}}_{i}^{\prime} \mathbf{F}_{i}^{*} \widehat{\mathbf{X}}_{i} \boldsymbol{\xi}+\sum_{i=1}^{k} \varrho_{n i}^{\prime} \mathbf{F}^{*} \widehat{\mathbf{X}}_{i} \boldsymbol{\xi}+\frac{1}{2} \varrho_{n i}^{\prime} \mathbf{F}^{*} \varrho_{n i} \\
& =: \mathbf{l}_{\mathbf{3}}^{\mathbf{1}}(\boldsymbol{\xi})+\mathbf{l}_{\mathbf{3}}^{\mathbf{2}}(\boldsymbol{\xi})+\mathbf{l}_{\mathbf{3}}^{\mathbf{3}}(\boldsymbol{\xi}) .
\end{aligned}
$$

By assumptions $\mathbf{A} \mathbf{3}$ and $\mathbf{A} 5$, we have that $\sup _{\mathbf{a}^{\prime} \mathbf{a}=\mathbf{1}}\left\|\mathbf{F}_{i}^{*}\right\|=O_{p}\left(n^{-1 / 2} q^{1 / 2}\right)$. Hence

$$
\begin{gathered}
\sup _{\|\boldsymbol{\xi}\| \leq L q^{1 / 2}, \mathbf{a}^{\prime} \mathbf{a}=\mathbf{1}}\left|\mathbf{1}_{\mathbf{3}}^{\mathbf{1}}(\boldsymbol{\xi})\right|=O_{p}\left(n^{-1 / 2} q^{5 / 2}\right), \\
\sup _{\|\boldsymbol{\xi}\| \leq L q^{1 / 2}, \mathbf{a}^{\prime} \mathbf{a}=\mathbf{1}}\left|\mathbf{l}_{\mathbf{3}}^{\mathbf{2}}(\boldsymbol{\xi})\right|=O_{p}\left(q^{3 / 2-h}\right), \\
\sup _{\|\boldsymbol{\xi}\| \leq L q^{1 / 2}, \mathbf{a}^{\prime} \mathbf{a}=\mathbf{1}}\left|\mathbf{l}_{\mathbf{3}}^{\mathbf{3}}(\boldsymbol{\xi})\right|=O_{p}\left(n^{-1 / 2} q^{1 / 2-2 h}\right) .
\end{gathered}
$$

Thus we have

$$
\sup _{\|\boldsymbol{\xi}\| \leq L q^{1 / 2}, \mathbf{a}^{\prime} \mathbf{a}=\mathbf{1}}\left|\mathbf{l}_{\mathbf{3}}\right|=O_{p}\left(q^{1 / 2}\right) .
$$

Putting all the approximations together, we have

$$
\sup _{\|\xi\| \leq L q^{1 / 2}}\|\boldsymbol{\psi}(\boldsymbol{\xi})-\boldsymbol{\Psi}(\boldsymbol{\xi})\|=O_{p}\left(q^{1 / 2}\right)
$$


and for sufficiently large $L$ and $C$, direct calculations give

$$
\begin{array}{rl}
\mathbf{E}\|\tilde{\boldsymbol{\xi}}\|^{2}=\sum_{i=1}^{k} & \mathbf{E}\left\{\left(\mathbf{y}_{i}-\mathbf{p}_{o i}\right)^{\prime} \mathbf{V}_{o i}^{-1} \boldsymbol{\Delta}_{o i} \tilde{\mathbf{X}} \mathbf{H}_{n}^{-1} \tilde{\mathbf{X}}_{i}^{\prime} \boldsymbol{\Delta}_{o i} \mathbf{V}_{o i}^{-1}\left(\mathbf{y}_{i}-\mathbf{p}_{o i}\right)\right. \\
\left.+\sum_{i=1}^{k} q\left(\mathbf{y}_{i}-\mathbf{p}_{o i}\right)^{\prime} \mathbf{V}_{o i}^{-1} \boldsymbol{\Delta}_{o i} \boldsymbol{\Pi}_{i} \mathbf{K}_{n}^{-2} \boldsymbol{\Pi}_{i}^{\prime} \boldsymbol{\Delta}_{o i} \mathbf{V}_{o i}^{-1}\left(\mathbf{y}_{i}-\mathbf{p}_{o i}\right)\right\} \\
\leq \operatorname{Ctrace}\left(\tilde{\mathbf{X}} \mathbf{H}_{n}^{-1} \tilde{\mathbf{X}}^{\prime}+q \boldsymbol{\Pi} \mathbf{K}_{n}^{-2} \Pi^{\prime}\right)=O(q) .
\end{array}
$$

Therefore,

$$
\sup _{\|\boldsymbol{\xi}\| \leq L q^{1 / 2}}\|\boldsymbol{\psi}(\boldsymbol{\xi})-\boldsymbol{\xi}\| \leq \sup _{\|\boldsymbol{\xi}\| \leq L q^{1 / 2}}\|\boldsymbol{\psi}(\boldsymbol{\xi})-\boldsymbol{\Psi}(\boldsymbol{\xi})\|+\|\tilde{\boldsymbol{\xi}}\|=L O_{p}\left(q^{1 / 2}\right)+O_{p}\left(q^{1 / 2}\right)
$$

which shows that for sufficiently large $L, \sup _{\|\boldsymbol{\xi}\| \leq L q^{1 / 2}}\|\boldsymbol{\psi}(\boldsymbol{\xi})-\boldsymbol{\xi}\| \leq L q^{1 / 2}$ in probability. Thus the theorem of Brouwer's fixed point ensures that there exists a zero $\hat{\boldsymbol{\xi}}$ of $\boldsymbol{\psi}(\boldsymbol{\xi})$ with $\|\hat{\boldsymbol{\xi}}\|=O_{p}\left(q^{1 / 2}\right)$ and hence the optimal convergence rate of the estimator of the non parametric function can be achieved. Similarly applying the Central Limit theorem on $\tilde{\boldsymbol{\xi}}_{\mathbf{1}}$, the asymptotic normality of the estimator $\hat{\boldsymbol{\beta}}$ can be established.

\subsubsection{Simulation Study}

We ran two sets of simulations to study the empirical properties of the proposed semiparametric method for estimating the marginal mean response and association parameters under binary longitudinal data.

\subsubsection{Response Models}

In first set of simulations, the marginal mean response $E\left(y_{i t} \mid \mathbf{x}_{i t}, z_{i t}, \boldsymbol{\beta}^{*}, \alpha\right)$ was defined as

$$
\operatorname{logit}\left[E\left(y_{i t} \mid \mathbf{x}_{i t}, z_{i t}, \boldsymbol{\beta}^{*}, \alpha\right)\right]=\beta_{1} x_{i}+m\left(z_{i t}\right),
$$

where $m\left(z_{i t}\right)$ was chosen as a nonlinear function, for $1 \leq i \leq k ; 1 \leq t \leq T$, and $x_{i}$ is a dichotomous covariate representing the treatment group for the $i^{\text {th }}$ individ- 
ual, with $P\left(x_{i}=1\right)=0.5$. The covariate $z_{i t}$ were produced from uniform $U[0,1]$. Parameters were fixed at $\beta_{1}=1, \alpha=0.3$. Each subject was observed at three timepoints $(T=3)$. In the second set of simulation study, the marginal mean response $E\left(y_{i t} \mid \mathbf{x}_{i t}, z_{i t}, \boldsymbol{\beta}^{*}, \alpha\right)$ was given by

$$
\operatorname{logit}\left[E\left(y_{i t} \mid \mathbf{x}_{i t}, z_{i t}, \boldsymbol{\beta}^{*}, \alpha\right)\right]=\beta_{1} x_{1 i}+\beta_{2} x_{2 i t}+m\left(z_{i t}\right)
$$

where $\beta_{1}=0.2, \beta_{2}=-0.2$. Values of $z_{i t}$ were generated from uniform $[0,1]$ and values of $x_{1 i}$ were generated from Bernoulli with the probability of success 0.5. Values of $x_{2 i t}$ were generated from $N(0,1)$.

For the first set of simulations, we chose $m()=.\theta+\sin (\pi(1-z))$, with $\theta=-1$, while for the second set, we chose $m()=.\sin (8 * z)$. We employed the proposed technique of spline regression for approximating $m(z)$ based on the linear spline regression with four knots for the first set and quadratic spline regression with six knots for the second set of the simulation study. The longitudinal binary data were obtained using a multivariate binary model as suggested by Bahadur (1961). Each Simulation run was based on 1000 replications of data sets, with each data set containing $k=300$ subjects and $T=3$ observations per subject. All the simulated data were obtained under the exchangeable correlation structure $\operatorname{Corr}\left(y_{i t}, y_{i t^{\prime}}\right)=$ 0.3 , for all $\left(t \neq t^{\prime}=1, \ldots, T\right)$.
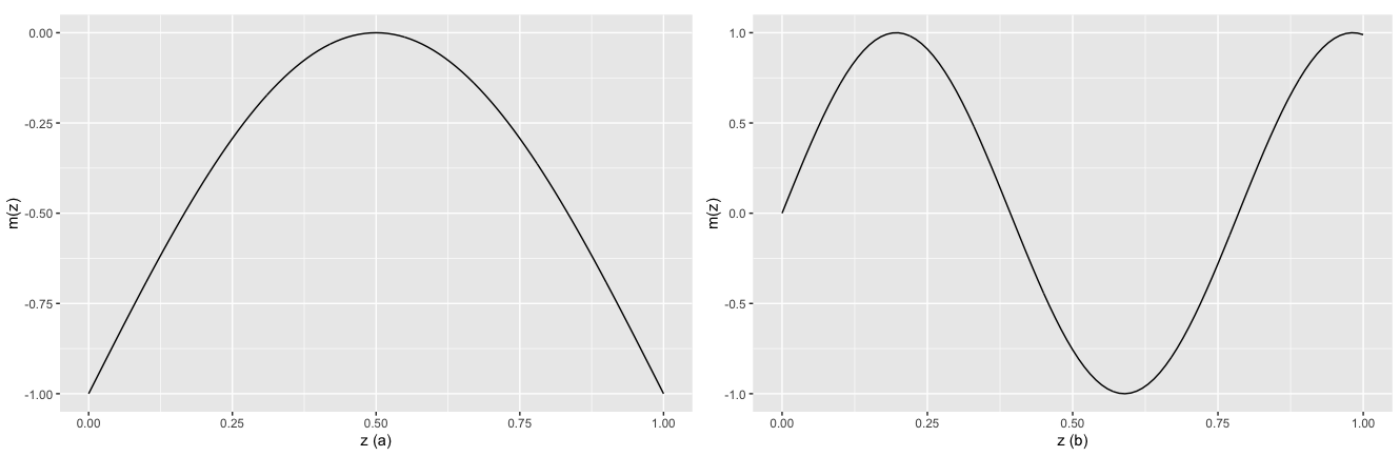

Figure 4.1: Nonlinear functions, (a) $m(z)=1+\sin (\pi(1-z))$ and (b) $m(z)=$ $\sin (8 * z)$, as used in the simulation study. 


\subsubsection{Results}

Tables 4.1 and 4.2 present empirical biases and mean squared errors of ordinary GEE estimators (GEE) assuming a linear function for $m(z)$ and our proposed regression spline GEE (RSGEE) estimators of the regression parameters $\left(\beta_{1}, \beta_{2}\right)$, correlation parameter $\alpha$ and nonlinear function $m(z)$ at different values of $z$ at $z=0.03,0.32,0.55,0.77$, and 0.99 for the first set and at $z=0,0.2,0.5,0.7$, and 0.9 for the second set of simulations.

Table 4.1: Empirical biases and mean squared errors (MSEs) of ordinary GEE and proposed RSGEE estimators when $m(z)=\theta+\sin (\pi(1-z)) ; z=$ $0.03,0.32,0.55,0.77,0.99$.

\begin{tabular}{ccccccc}
\hline \hline \multirow{2}{*}{ Parameter } & & \multicolumn{2}{c}{$G E E$} & & \multicolumn{2}{c}{$R S G E E$} \\
\cline { 3 - 4 } & True value & Bias & MSE & & Bias & MSE \\
\hline \hline$\beta_{1}$ & 1 & -0.020 & 0.029 & & 0.008 & 0.030 \\
$\alpha$ & 0.3 & -0.009 & 0.001 & & -0.001 & 0.001 \\
$m\left(z_{1}\right)$ & -0.91 & 0.548 & 0.326 & & -0.008 & 0.066 \\
$m\left(z_{2}\right)$ & -0.15 & -0.202 & 0.057 & & -0.017 & 0.025 \\
$m\left(z_{3}\right)$ & -0.01 & -0.346 & 0.134 & & -0.006 & 0.028 \\
$m\left(z_{4}\right)$ & -0.34 & -0.019 & 0.018 & & -0.003 & 0.037 \\
$m\left(z_{5}\right)$ & -0.97 & 0.609 & 0.397 & & -0.013 & 0.082 \\
\hline
\end{tabular}

Table 4.2: Empirical biases and mean squared errors (MSEs) of ordinary GEE and proposed RSGEE estimators when $m(z)=\sin (8 * z) ; z=0,0.2,0.5,0.7,0.9$.

\begin{tabular}{cccccc}
\hline \hline & & \multicolumn{2}{c}{$G E E$} & \multicolumn{2}{c}{$R S G E E$} \\
\cline { 3 - 6 } Parameter & True value & Bias & MSE & Bias & MSE \\
\hline \hline & & & & & \\
$\beta_{1}$ & 0.1 & -0.009 & 0.020 & 0.003 & 0.021 \\
$\beta_{2}$ & 0.1 & -0.011 & 0.004 & 0.001 & 0.005 \\
$\alpha$ & 0.2 & -0.019 & 0.002 & 0.003 & 0.001 \\
$m\left(z_{1}\right)$ & 0.00 & 0.363 & 0.156 & 0.016 & 0.259 \\
$m\left(z_{2}\right)$ & 0.99 & -0.727 & 0.545 & 0.018 & 0.055 \\
$m\left(z_{3}\right)$ & -0.76 & 0.892 & 0.807 & -0.021 & 0.051 \\
$m\left(z_{4}\right)$ & -0.63 & 0.675 & 0.471 & -0.012 & 0.038 \\
$m\left(z_{5}\right)$ & 0.79 & -0.841 & 0.728 & 0.012 & 0.076 \\
\hline
\end{tabular}

It is clear from Tables 4.1 and 4.2 that the ordinary GEE method provides biased estimates of the nonlinear function $m(z)$ at different values of $z$. The proposed RSGEE method appears to perform much better than the GEE method in 
terms of both bias and mean squared errors of the estimators. For example, when estimating $m\left(z_{1}=0.03\right)$, the GEE method provides a percentage relative bias of $(0.548 / 0.91) 100=60.2 \%$ and a mean squared error of 0.326 , whereas the proposed RSGEE method provides a much smaller bias of $(0.008 / 0.91) 100=0.9 \%$ and a smaller mean squared error of 0.066 , as shown in Table 4.1. Also, Table 4.2 shows that when estimating $m\left(z_{5}=0.9\right)$, the GEE method provides a large percentage relative bias of $(0.841 / 0.79) 100=106.5 \%$ and mean squared error of 0.728 , whereas the proposed RSGEE method provides a much smaller bias of $(0.012 / 0.79) 100=1.5 \%$ and a small mean squared error of 0.076 .

\subsection{Partially Linear Count Longitudinal Model}

\subsubsection{Response Models}

\subsubsection{Log-Linear Model}

Let the data comprise of $k$ subjects with each subject being studied at a fixed set of $T$ time points. Let $y_{i t}$ be the count response variable and $\mathbf{x}_{i t}$ be a $p \times 1$ vector of covariates measured at the $t^{t h}$ time point on the $i^{\text {th }}$ subject. The covariates here may be fixed or time dependent through the whole observation times.

Assume the marginal distribution for the count response variable $y_{i t}$ is

$$
y_{i t} \sim \operatorname{Poisson}\left(\mu_{i t}\right), \quad i=1, \ldots, k, \quad t=1, \ldots, T
$$

with the marginal mean and variance functions

$$
\begin{gathered}
E\left(y_{i t} \mid \mathbf{X}_{i}\right)=\mu_{i t}, \\
\operatorname{var}\left(y_{i t} \mid \mathbf{X}_{i}\right)=\phi \mu_{i t},
\end{gathered}
$$

where $\phi$ is a dispersion parameter. Similarly to Sullivan Pepe and Anderson (1994), assume that the mean response for the variable $y_{i t}$ depends only on the vector of 
covariates for subject $i$ at time $t$, i.e., $\mu_{i t}=E\left(y_{i t} \mid \mathbf{X}_{i}\right)=E\left(y_{i t} \mid \mathbf{x}_{i t}\right)$.

We assume a log-linear model for mean response $\mu_{i t}$, given by

$$
\log \left(\mu_{i t}\right)=\mathbf{x}_{i t}^{\prime} \boldsymbol{\beta}
$$

where $\boldsymbol{\beta}$ is a $p \times 1$ vector of regression parameters. Assume that $y_{i t}$ and $y_{i^{\prime} t^{\prime}}$ are uncorrelated when $i \neq i^{\prime}$, but observations from a given subject are correlated as

$$
\operatorname{corr}\left(y_{i t}, y_{i t^{\prime}}\right)=\alpha_{t t^{\prime}}
$$

where $\boldsymbol{\alpha}=\left(\alpha_{12}, \ldots, \alpha_{1 T}, \alpha_{23}, \ldots, \alpha_{T-1, T}\right)^{\prime}$ represents a vector of correlation parameters.

\subsubsection{Partially Log-Linear Model}

We now consider some covariate $z_{i t}$ that is related to the mean response $\mu_{i t}$ through an unknown function $m\left(z_{i t}\right)$. In particular, we consider a partially log-linear model in the form

$$
\log \left(\mu_{i t}\right)=\mathbf{x}_{i t}^{\prime} \boldsymbol{\beta}+m\left(z_{i t}\right)
$$

where $\boldsymbol{\beta}$ is a $p \times 1$ vector of regression parameters and $m\left(z_{i t}\right)$ is an unknown smooth function. The idea is to approximate the unknown function $m($.$) by the spline$ regression, so that model (4.54) is approximated as

$$
\log \left(\mu_{i t}\right)=\mathbf{x}_{i t}^{\prime} \boldsymbol{\beta}+\Phi_{i t}^{\prime} \boldsymbol{\gamma}=\mathbf{x}_{i t}^{*^{\prime}} \boldsymbol{\beta}^{*}
$$

where $\boldsymbol{\beta}^{*}=(\boldsymbol{\beta}, \boldsymbol{\gamma})$, and $\Phi$ is the truncated power basis function along the spline vector coefficient $\gamma$. This model has the same marginal variance and correlation structure as (4.50) and (4.51). If $m($.$) is stated as the identity function, then model$ (4.55) becomes a typical log-linear regression like (4.52). Here we model the effects of covariates $\mathbf{X}$ parametrically and that of $\mathbf{Z}$ nonparametrically. 


\subsubsection{Methods of Estimation}

\subsubsection{Generalized Estimating Equations}

The main objective is to estimate the regression parameters $\boldsymbol{\beta}^{*}=(\boldsymbol{\beta}, \boldsymbol{\gamma})$, association parameters $\boldsymbol{\alpha}$ and dispersion parameter $\phi$. As a naive technique, longitudinal data are analyzed by assuming an identity function for $m($.$) , where the model pa-$ rameters are estimated based on the generalized estimating equations (GEEs) of Liang and Zeger (1986). The regression parameters $\boldsymbol{\beta}^{*}$ may be estimated by solving

$$
\begin{gathered}
\mathbf{U}_{\boldsymbol{\beta}^{*}}\left(\boldsymbol{\beta}^{*}, \boldsymbol{\alpha}\right)=\sum_{i=1}^{k} \mathbf{D}_{i}^{\prime} \mathbf{V}_{i}^{-1}\left(\mathbf{y}_{i}-\boldsymbol{\mu}_{i}\left(\boldsymbol{\beta}^{*}\right)\right) \\
=\sum_{i=1}^{k} \mathbf{X}_{i}^{*^{\prime}} \boldsymbol{\Delta}_{i}\left(\boldsymbol{\mu}_{i}\left(\boldsymbol{\beta}^{*}\right)\right) \mathbf{V}_{i}^{-1}\left(\boldsymbol{\mu}_{i}\left(\boldsymbol{\beta}^{*}, \boldsymbol{\alpha}\right)\right)\left(\mathbf{y}_{i}-\boldsymbol{\mu}_{i}\left(\boldsymbol{\beta}^{*}\right)\right)=0
\end{gathered}
$$

with respect to $\boldsymbol{\beta}^{*}$, where $\mathbf{D}_{i}=\partial \boldsymbol{\mu}_{i} / \partial \boldsymbol{\beta}^{*}=\boldsymbol{\Delta}_{i} \mathbf{X}_{i}^{*}, \quad \boldsymbol{\Delta}_{i}\left(\boldsymbol{\mu}_{i}\left(\boldsymbol{\beta}^{*}\right)\right)=\partial \boldsymbol{\mu}_{i} / \partial \boldsymbol{\eta}_{i}=$ $\operatorname{diag}\left\{\dot{\mu}_{i 1}\left(\boldsymbol{\beta}^{*}\right), \ldots, \dot{\mu}_{i t}\left(\boldsymbol{\beta}^{*}\right)\right\}$, with $\dot{\mu}_{i t}($.$) being the first derivative of \boldsymbol{\mu}($.$) evaluated$ at $\boldsymbol{\beta}^{*}, \mathbf{V}_{i}=\phi\left(\mathbf{A}_{i}\right)^{1 / 2} \boldsymbol{\rho}(\boldsymbol{\alpha})\left(\mathbf{A}_{i}\right)^{1 / 2}$, with $\mathbf{A}_{i}=\operatorname{diag}\left\{\mu_{i 1}, \mu_{i 2} \ldots, \mu_{i T}\right\}$, and $\boldsymbol{\rho}(\boldsymbol{\alpha})$ is a working correlation matrix for $\mathbf{y}_{\mathbf{i}}$, depending on the vector of correlation parameters $\boldsymbol{\alpha}$. For the estimates of $\boldsymbol{\beta}^{*}$, an iterative method is used to solve the above equation. If $\boldsymbol{\alpha}$ and $\phi$ were known, then the solution to equation (4.56) is efficient. For estimating $\boldsymbol{\alpha}$ and $\phi$, Liang and Zeger (1986) suggested the method of moments based on Pearson residuals

$$
\hat{r}_{i t}=\frac{\left(y_{i t}-\hat{\mu}_{i t}\right)}{\sqrt{\left(\hat{\mu}_{i t}\right)}} .
$$

The moment estimators of $\alpha_{t t^{t}}$ are obtained as

$$
\hat{\alpha}_{t t^{\prime}}=\frac{1}{\hat{\phi}} \sum_{i=1}^{k} \hat{r}_{i t} \hat{r}_{i t}^{\prime} / k-N_{n},
$$

and $\phi$ can be estimated by

$$
\hat{\phi}=\sum_{i=1}^{k} \sum_{t=1}^{T}, \hat{r}_{i t}^{2} /\left(N-N_{n}\right)
$$


where $N_{n}=p+N_{q}$ is the dimension of $\boldsymbol{\beta}^{*}$.

The estimator $\hat{\boldsymbol{\beta}}^{*}$ is asymptotically normal and consistent under weak situations even when $\boldsymbol{\alpha}$ and $\phi$ are substituted by $k^{1 / 2}$ - consistent estimators. To permit joint approximation of the regression and association parameters, an extension of the GEE method is considered by Prentice (1988). We know that $\mathbf{U}_{\boldsymbol{\beta}^{*}}$ depends not only on $\boldsymbol{\beta}^{*}$ and $\boldsymbol{\alpha}$, but also on $\phi$ because of the need to account for overdispersion. Therefore, let $\boldsymbol{\vartheta}=\left(\boldsymbol{\alpha}^{\prime}, \phi\right)^{\prime}$ be the vector of association with dimension $q=q_{1}+q_{2}$, where $q_{1}$ and $q_{2}$ are the dimensions of $\boldsymbol{\alpha}$ and $\phi$. Following Prentice (1988), we estimate $\boldsymbol{\vartheta}$ by solving

$$
\mathbf{U}_{\boldsymbol{\vartheta}}\left(\boldsymbol{\beta}^{*}, \boldsymbol{\vartheta}\right)=\sum_{i=1}^{k} \mathbf{L}_{i}^{\prime} \mathbf{W}_{i}^{-1}\left(\mathbf{r}_{i}-\boldsymbol{\delta}_{i}\right)=0
$$

where $\mathbf{r}_{i}=\left(r_{i 1} r_{i 2}, r_{i 1} r_{i 3}, \ldots, r_{i, T-1} r_{i, T}, r_{i 1}^{2}, r_{i 2}^{2}, \ldots, r_{i T}^{2}\right)^{\prime}$, with

$$
r_{i t}=\frac{\left(y_{i t}-\mu_{i t}\right)}{\sqrt{\mu_{i t}}}
$$

$\boldsymbol{\delta}_{i}=E\left(\mathbf{r}_{i}\right)$, and $\mathbf{W}_{i}=\operatorname{diag}\left\{\boldsymbol{v a r}\left(\mathbf{r}_{i}\right)\right\}$. GEE estimators $\left(\boldsymbol{\beta}^{*}, \boldsymbol{\vartheta}\right)$ may be obtained by simultaneously solving the estimating equations

$$
\begin{gathered}
\mathbf{U}_{\boldsymbol{\beta}^{*}}\left(\boldsymbol{\beta}^{*}, \boldsymbol{\vartheta}\right)=\sum_{i=1}^{k} \mathbf{D}_{i}^{\prime} \mathbf{V}_{i}^{-1}\left(\mathbf{y}_{i}-\boldsymbol{\mu}_{i}\right)=0 \\
\mathbf{U}_{\boldsymbol{\vartheta}}\left(\boldsymbol{\beta}^{*}, \boldsymbol{\vartheta}\right)=\sum_{i=1}^{k} \mathbf{L}_{i}^{\prime} \mathbf{W}_{i}^{-1}\left(\mathbf{r}_{i}-\boldsymbol{\delta}_{i}\right)=0
\end{gathered}
$$

where $\mathbf{L}_{i}=\partial \boldsymbol{\delta}_{i} / \partial \boldsymbol{\vartheta}$

\subsubsection{Estimating Algorithm}

Recall the count response $y_{i t}$, where the mean response $\mu_{i t}$ is approximated by

$$
\log \left(\mu_{i t}\right)=\mathbf{x}_{i t}^{*^{\prime}} \boldsymbol{\beta}^{*}
$$

with

$$
\mathbf{x}_{i t}^{*^{\prime}} \boldsymbol{\beta}^{*}=\mathbf{x}_{i t}^{\prime} \boldsymbol{\beta}+\Phi_{i t}^{\prime} \boldsymbol{\gamma}
$$


The regression parameters $\boldsymbol{\beta}^{*}$ and the association parameters $\boldsymbol{\vartheta}$ are estimated by solving the equations

$$
\mathbf{U}_{\boldsymbol{\beta}^{*}}\left(\boldsymbol{\beta}^{*}, \boldsymbol{\vartheta}, m(.)\right)=\sum_{i=1}^{k} \mathbf{D}_{i}^{\prime} \mathbf{V}_{i}^{-1}\left(\mathbf{y}_{i}-\boldsymbol{\mu}\left(\mathbf{X}_{i}^{*} \boldsymbol{\beta}^{*}\right)\right)=0
$$

and

$$
\mathbf{U}_{\boldsymbol{\vartheta}}\left(\boldsymbol{\beta}^{*}, \boldsymbol{\vartheta}, m(.)\right)=\sum_{i=1}^{k} \mathbf{L}_{i}^{\prime} \mathbf{W}_{i}^{-1}\left(\mathbf{r}_{i}-\boldsymbol{\delta}_{i}\right)=0,
$$

where $\hat{\boldsymbol{\beta}}$ estimates the parametric part of the model and $\hat{m}()=.\Phi^{\prime}(.) \hat{\gamma}$ estimates the non parametric part of the model.

The diagonal matrix $\mathbf{W}_{i}$ is often set to be the identity matrix $\mathbf{I}$ to avoid estimating additional higher order parameters and to reduce sampling variation (Diggle et al., 2002). Our computational experience suggests that the choice $\mathbf{W}=\mathbf{I}$ leads to loss of efficiency in estimating $\boldsymbol{\vartheta}$, but it has very little impact on the estimation of $\boldsymbol{\beta}^{*}$.

We describe below an algorithm for simultaneously estimating the regression parameters $\boldsymbol{\beta}^{*}$, correlation parameters $\boldsymbol{\alpha}$ and dispersion parameter $\phi$.

Step 0. Consider some initial values $\left(\hat{\boldsymbol{\beta}}_{0}^{*}, \hat{\vartheta}_{0}\right)$.

Step 1. For a given number and locations of knots, approximate $m\left(z_{i t}\right)$ by $\hat{m}\left(z_{i t}\right)=\sum_{j=0}^{l} \hat{\gamma}_{j o} z_{i t}^{j}+\sum_{r=1}^{q} \hat{\gamma}_{l+r, o}\left(z_{i t}-\zeta_{r}\right)_{+}^{l}$.

Step 2. Given the approximate $\hat{m}(z)$, and initial estimates of $\hat{\boldsymbol{\beta}}_{0}^{*}$ and $\hat{\boldsymbol{\vartheta}}_{0}$, update $\boldsymbol{\beta}^{*}$ and $\boldsymbol{\vartheta}$ by solving the equations

$$
\mathbf{U}_{\boldsymbol{\beta}^{*}}\left(\boldsymbol{\beta}^{*}, \boldsymbol{\vartheta}, m(.)\right)=\sum_{i=1}^{k} \mathbf{D}_{i}^{\prime} \mathbf{V}_{i}^{-1}\left(\mathbf{y}_{i}-\boldsymbol{\mu}_{i}\left(\boldsymbol{\beta}^{*}, \boldsymbol{\vartheta}\right)\right)=0
$$

for $\boldsymbol{\beta}^{*}$, and

$$
\mathbf{U}_{\vartheta}\left(\boldsymbol{\beta}^{*}, \boldsymbol{\vartheta}, m(.)\right)=\sum_{i=1}^{k} \mathbf{L}_{i}^{\prime} \mathbf{W}_{i}^{-1}\left(\mathbf{r}_{i}-\boldsymbol{\delta}_{i}\right)=0,
$$

for $\boldsymbol{\vartheta}$, where $\boldsymbol{\mu}_{i}\left(\boldsymbol{\beta}^{*}, \boldsymbol{\vartheta}\right)=\left(\mu_{i 1}\left(\boldsymbol{\beta}^{*}, \boldsymbol{\vartheta}\right), \ldots, \mu_{i T}\left(\boldsymbol{\beta}^{*}, \boldsymbol{\vartheta}\right)\right)^{\prime}$ is the vector of mean responses with

$$
\mu_{i t}\left(\boldsymbol{\beta}^{*}, \boldsymbol{\vartheta}\right)=\exp \left(\mathbf{x}_{i t}^{\prime} \boldsymbol{\beta}+\hat{m}\left(z_{i t}\right)\right)
$$


Step 3. Repeat steps 1 and 2 until convergence in $\hat{\boldsymbol{\beta}}^{*}$ and $\hat{\boldsymbol{\vartheta}}$.

When executing the above algorithm it is practical to choose an initial value by the GEE method and assuming the identity function for $m($.$) .$

\subsubsection{Computational Details for Estimating the Response Model}

Recall the algorithm discussed in section 4.5.2.2. In Step 1, we approximate the nonlinear function $m\left(z_{i t}\right)$ by the spline regression

$$
\hat{m}\left(z_{i t}\right)=\Phi_{i t}^{\prime} \gamma
$$

for which the mean response $\mu_{i t}$ is approximated by

$$
\log \left(\mu_{i t}\right)=\mathbf{x}_{i t}^{*^{\prime}} \boldsymbol{\beta}^{*}=\mathbf{x}_{i t}^{\prime} \boldsymbol{\beta}+\Phi_{i t}^{\prime} \boldsymbol{\gamma}
$$

The iterative technique starts with some initial values $\left(\hat{\boldsymbol{\beta}}_{0}^{*}, \hat{\boldsymbol{\vartheta}}_{0}\right)$, and produces updated values $\hat{\boldsymbol{\beta}}_{s+1}^{*}, \hat{\boldsymbol{\vartheta}}_{s+1}$ from $\hat{\boldsymbol{\beta}}_{s}^{*}, \hat{\boldsymbol{\vartheta}}_{s}$ by means of the iterative equations

$$
\hat{\boldsymbol{\beta}}_{s+1}^{*}=\hat{\boldsymbol{\beta}}_{s}^{*}+\left(\sum_{i=1}^{k} \mathbf{D}_{i}^{\prime} \mathbf{V}_{i}^{-1} \mathbf{D}_{i}\right)^{-1} \sum_{i=1}^{k} \mathbf{D}_{i}^{\prime} \mathbf{V}_{i}^{-1}\left(\mathbf{y}_{i}-\boldsymbol{\mu}_{i}\left(\boldsymbol{\beta}^{*}, \boldsymbol{\alpha}, \phi\right)\right),
$$

and

$$
\hat{\boldsymbol{\vartheta}}_{s+1}=\hat{\boldsymbol{\vartheta}}_{s}+\left(\sum_{i=1}^{k} \mathbf{L}_{i}^{\prime} \mathbf{W}_{i}^{-1} \mathbf{L}_{i}\right)^{-1} \sum_{i=1}^{k} \mathbf{L}_{i}^{\prime} \mathbf{W}_{i}^{-1}\left(\mathbf{r}_{i}-\boldsymbol{\delta}_{i}\right),
$$

for $s=0,1,2, \ldots$, where the second term on the right side of each estimating equation is calculated at the current estimates $\left(\hat{\boldsymbol{\beta}}_{s}^{*}, \hat{\boldsymbol{\vartheta}}_{s}\right)$.

\subsubsection{Simulation Study}

To assess the performance of the proposed regression spline GEE (RSGEE) method, we ran two sets of simulations using count longitudinal data generated from a partially log-linear model. 


\subsubsection{Generation of Correlated Counts:}

To generate correlated count longitudinal data, here we use a Poisson mixed model in the form

- $y_{i t} \mid b_{i} \sim$ ind.Poisson $\left(\mu_{i t}\right)$,

- $\log \left(\mu_{i t}\right)=\mathbf{x}_{i t}^{\prime} \boldsymbol{\beta}+m\left(z_{i t}\right)+b_{i}$,

- $b_{i} \sim \operatorname{Normal}\left(0, \sigma^{2}\right), \quad$ for $i=1, \ldots, N, t=1, \ldots, T$, and $\sigma=[0.3]$.

We generated a large data set with $N=500,000$ subjects form this mixed model. For this given data set, the GEE estimates under the exchangeable correlation structure were treated as the true population parameters. We then ran our simulations by generating random sample from this "Population" with each combination of $k=300$ and $k=500$ subjects.

\subsubsection{Response Models}

The marginal mean response model is given by

$$
\log \left[E\left(y_{i t} \mid \mathbf{x}_{i t}, \boldsymbol{\beta}, \alpha, \phi\right)\right]=\beta_{1} x_{i}+\beta_{2} t+m\left(z_{i t}\right),
$$

for $i=1, \ldots, k$, and $t=1, \ldots, T$, where $x_{i}$ is a binary covariates representing the treatment group with $P\left(x_{i}=1\right)=0.5$ and $T=3$. The regression parameters were fixed at $\left(\beta_{1}, \beta_{2}\right)=(0.5,0.2)$. For the first set of simulations, we choose a linear function $m\left(z_{i t}\right)=0.5+0.2 z_{i t}$ and for the second set, we choose $m\left(z_{i t}\right)=$ $0.5+0.2 z_{i t} /\left(0.05+z_{i t}\right)$, where $z_{i t}$ is generated from the uniform $(0,1)$ distribution. We employed the proposed technique of spline regression for approximating $m(z)$ based on the linear spline regression with six knots in both simulations. Figure 4.2 exhibits the two functions (linear vs. nonlinear $m($.$) ), as used in the simulation$ study. Each simulation ran was based on 1000 replicates of data sets. 

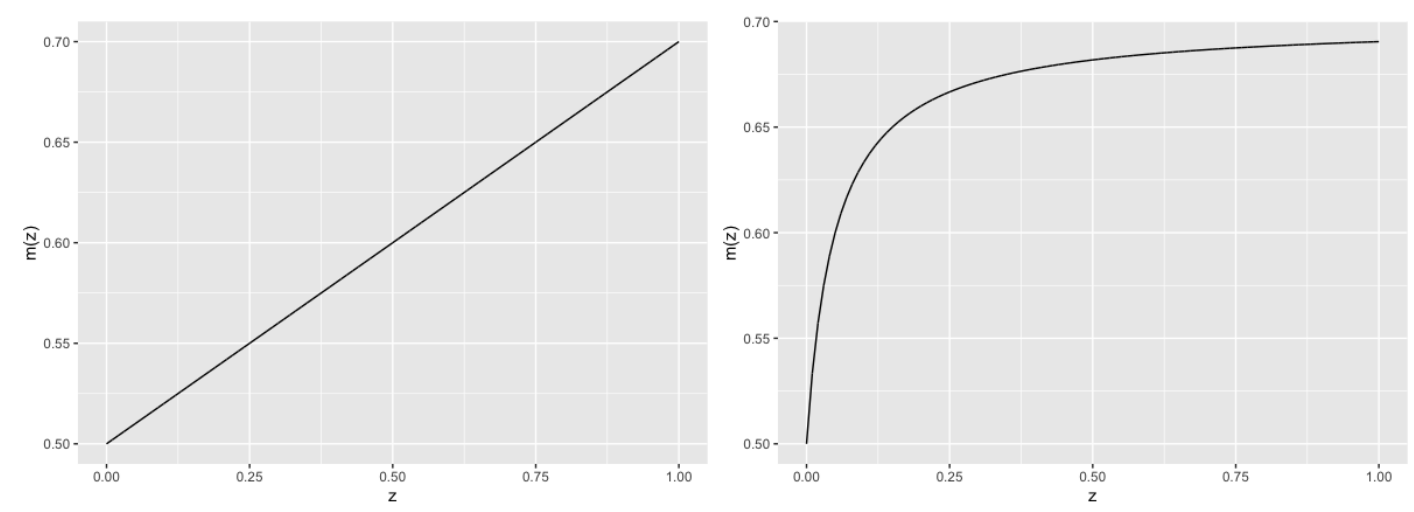

Figure 4.2: $z$ vs. $m(z)$ for linear and nonlinear functions, as used in the simulation study

\subsubsection{Results}

Tables 4.3 and 4.4 present empirical biases and mean squared errors of ordinary GEE estimators (GEE) assuming a linear function for $m(z)$ and our proposed regression spline GEE (RSGEE) estimators of the regression parameters $\left(\beta_{1}, \beta_{2}\right)$, correlation parameter $\alpha$, dispersion parameters $\phi$ and nonlinear function $m(z)$ at different values of $z$ at $m(z)$, at $z=0.01,0.05,0.11,0.66,0.99$. Table 4.3 present the results for the case when $m(z)$ is, in fact, linear. Table 4.4 shows the results when true $m(z)$ is a nonlinear function. 
Table 4.3: Biases and MSEs under Prentice's second-order GEE and proposed method RSGEE. True $m(z)=0.5+0.2 z$.

Linear Model, $\mathrm{k}=300$

\begin{tabular}{ccccccc}
\hline \hline & & \multicolumn{2}{c}{ GEE } & & \multicolumn{2}{c}{ RSGEE } \\
Parameter & True value & Bias & MSE & & Bias & MSE \\
\hline \hline$\beta_{1}$ & 0.5 & -0.0003 & 0.0016 & & -0.0005 & 0.0016 \\
$\beta_{2}$ & 0.2 & -0.0001 & 0.0005 & & -0.0002 & 0.0005 \\
$\alpha$ & 0.28 & -0.0010 & 0.0019 & & 0.0015 & 0.0019 \\
$\phi$ & 1.42 & -0.0197 & 0.0068 & & -0.0283 & 0.0072 \\
$m\left(z_{1}\right)$ & 0.50 & 0.0506 & 0.0069 & & 0.0484 & 0.0131 \\
$m\left(z_{2}\right)$ & 0.51 & 0.0498 & 0.0067 & & 0.0487 & 0.0090 \\
$m\left(z_{3}\right)$ & 0.52 & 0.0505 & 0.0065 & & 0.0496 & 0.0083 \\
$m\left(z_{4}\right)$ & 0.63 & 0.0507 & 0.0060 & & 0.0484 & 0.0075 \\
$m\left(z_{5}\right)$ & 0.69 & 0.0501 & 0.0067 & & 0.0481 & 0.0087 \\
\hline
\end{tabular}

Linear Model, $\mathrm{k}=500$

\begin{tabular}{ccccccc}
\hline \hline & & \multicolumn{2}{c}{ GEE } & & \multicolumn{2}{c}{ RSGEE } \\
Parameter & True value & Bias & MSE & & Bias & MSE \\
\hline \hline$\beta_{1}$ & 0.5 & 0.0015 & 0.0009 & & 0.0014 & 0.0009 \\
$\beta_{2}$ & 0.2 & 0.0005 & 0.0003 & & 0.0005 & 0.0003 \\
$\alpha$ & 0.28 & 0.0000 & 0.0011 & & 0.0016 & 0.0011 \\
$\phi$ & 1.42 & -0.0184 & 0.0044 & & -0.0234 & 0.0046 \\
$m\left(z_{1}\right)$ & 0.50 & 0.0484 & 0.0050 & & 0.0471 & 0.0088 \\
$m\left(z_{2}\right)$ & 0.51 & 0.0483 & 0.0049 & & 0.0473 & 0.0060 \\
$m\left(z_{3}\right)$ & 0.52 & 0.0483 & 0.0047 & & 0.0476 & 0.0056 \\
$m\left(z_{4}\right)$ & 0.63 & 0.0478 & 0.0044 & & 0.0472 & 0.0051 \\
$m\left(z_{5}\right)$ & 0.69 & 0.0475 & 0.0050 & & 0.0473 & 0.0083 \\
\hline \hline
\end{tabular}


Table 4.4: Biases and MSEs under usual Prentice's second-order GEE and proposed RSGEE. True $m(z)=0.5+0.2 z /(0.05+z)$.

Partially Linear Model, $\mathrm{k}=300$

\begin{tabular}{ccccccc}
\hline \hline & & \multicolumn{2}{c}{ GEE } & & \multicolumn{2}{c}{ RSGEE } \\
Parameter & True value & Bias & MSE & & Bias & MSE \\
\hline \hline$\beta_{1}$ & 0.5 & -0.0007 & 0.0015 & & -0.0008 & 0.0014 \\
$\beta_{2}$ & 0.2 & -0.0003 & 0.0004 & & -0.0003 & 0.0004 \\
$\alpha$ & 0.3 & -0.0090 & 0.0019 & & -0.0041 & 0.0018 \\
$\phi$ & 1.43 & -0.0047 & 0.0072 & & -0.0124 & 0.0074 \\
$m\left(z_{1}\right)$ & 0.53 & 0.1483 & 0.0264 & & 0.0712 & 0.0158 \\
$m\left(z_{2}\right)$ & 0.60 & 0.0848 & 0.0114 & & 0.0356 & 0.0071 \\
$m\left(z_{3}\right)$ & 0.63 & 0.0521 & 0.0067 & & 0.0447 & 0.0072 \\
$m\left(z_{4}\right)$ & 0.68 & 0.0474 & 0.0056 & & 0.0495 & 0.0066 \\
$m\left(z_{5}\right)$ & 0.69 & 0.0692 & 0.0089 & & 0.0466 & 0.0117 \\
\hline
\end{tabular}

Partially Linear Model, $\mathrm{k}=\mathbf{5 0 0}$

\begin{tabular}{ccccccc}
\hline \hline & & \multicolumn{2}{c}{ GEE } & & \multicolumn{2}{c}{ RSGEE } \\
\cline { 6 - 7 } Parameter & True value & Bias & MSE & & Bias & MSE \\
\hline \hline$\beta_{1}$ & 0.5 & 0.0003 & 0.0008 & & -0.0004 & 0.0008 \\
$\beta_{2}$ & 0.2 & 0.0004 & 0.0002 & & 0.0003 & 0.0002 \\
$\alpha$ & 0.3 & -0.0054 & 0.0011 & & -0.0035 & 0.0011 \\
$\phi$ & 1.43 & -0.0006 & 0.0041 & & -0.0069 & 0.0042 \\
$m\left(z_{1}\right)$ & 0.53 & 0.1466 & 0.0240 & & 0.0693 & 0.0111 \\
$m\left(z_{2}\right)$ & 0.60 & 0.0832 & 0.0093 & & 0.0349 & 0.0050 \\
$m\left(z_{3}\right)$ & 0.63 & 0.0511 & 0.0049 & & 0.0451 & 0.0050 \\
$m\left(z_{4}\right)$ & 0.68 & 0.0468 & 0.0041 & & 0.0478 & 0.0051 \\
$m\left(z_{5}\right)$ & 0.69 & 0.0691 & 0.0072 & & 0.0503 & 0.0083 \\
\hline \hline
\end{tabular}

It is clear from Table 4.3 that when true $m(z)$ is a linear function, both GEE and RSGEE provide very close results in terms of both biases and mean squared errors of the estimates. Both method provide roughly unbiased estimators. However, the RSGEE method provides slightly larger mean squared errors as compared to the GEE method. This is expected here, since under the correctly specified linear $m($.$) function, the ordinary GEE method is expected to be more efficient than the$ RSGEE method. However, the RSGEE method appears to perform better when the true $m($.$) function is nonlinear, as shown in Table 4.4. For example, when$ $k=500$, for estimating $m\left(z_{1}\right)$, the GEE provides a percentage relative bias of $(0.1466 / 0.53) * 100=27.7 \%$ and an MSE of 0.0240, whereas the RSGEE method 
provides a smaller percentage relative bias of $(0.0693 / 0.53) * 100=13 \%$ and a smaller MSE of 0.0111.

\subsection{Conclusions}

The main objective of this study was to explore and suggest a semiparametric procedure for analyzing binary and count longitudinal data. Our simulation study reveals that if the underlying true model is partially linear, then our suggested technique usually produces an unbiased and efficient estimator. The proposed technique still produces a consistent estimator even if a nonparametric function is incorporated into the model, when the true marginal mean, in fact, has a linear form. Therefore, the technique described here has vast applications in a variety of settings. It can be generalized for accommodating data with complex associations among the response variable and its predictors. 


\section{Chapter 5}

\section{Semiparametric Longitudinal Model}

\section{With Missing Responses}

\subsection{Introduction}

In this chapter, the proposed semiparametric method is extended for analyzing incomplete longitudinal data with dropouts. To analyze incomplete data, we incorporate inverse probability weights of Robins et al. (1995) into the semiparametric method as proposed in Chapter 4.

The organization of this chapter is as follows: Section 5.2 introduces the notation and model to describe partially linear models for binary incomplete longitudinal data. Section 5.2.3 discusses the weighted GEE estimation of partially linear models, while Section 5.2.4 presents the asymptotic properties of the proposed estimators. Section 5.2.5 discusses finite sample properties of proposed estimators based on a simulation study. Section 5.2.6 presents an application using binary longitudinal data with dropouts. Section 5.3 describes the proposed weighted GEE method in the context of incomplete count longitudinal data. Section 5.3.3 addresses the empirical properties of proposed estimators based on a simulation study. 


\subsection{Partially Linear Model for Incomplete Binary Longitudinal Data}

\subsubsection{Logistic Response Model}

Suppose $k$ subjects $(i=1, \ldots, k)$ are studied at a fixed set of $T$ time points. Let $y_{i t}$ denote the response variable and $\left(\mathbf{x}_{i t}, z_{i t}\right)$ be the covariates from the $i^{\text {th }}$ subject measured at time t, where $\mathbf{x}_{i t}$ and $z_{i t}$ are a $p \times 1$ vector and a scalar, respectively. The covariates here may be fixed or time-dependent throughout the whole observation times.

Let $p_{i t}$ be the marginal probability of success at time $t$ for the $i^{\text {th }}$ individual. That is, assume

$$
y_{i t} \sim \operatorname{Bernoulli}\left(p_{i t}\right), \quad i=1, \ldots, k, \quad 1, \ldots, T
$$

with the probability of success

$$
p_{i t}=E\left(y_{i t} \mid \mathbf{X}_{i}, \mathbf{Z}_{i}\right)=P\left(y_{i t}=1 \mid \mathbf{X}_{i}, \mathbf{Z}_{i}\right)
$$

Assume that the mean of the response variable $y_{i t}$ depends only on the covariate vector for subject $i$ at time $t$, i.e., $p_{i t}=E\left(y_{i t} \mid \mathbf{X}_{i}, \mathbf{Z}_{i}\right)=E\left(y_{i t} \mid \mathbf{x}_{i t}, z_{i t}\right)$, (Sullivan Pepe and Anderson, 1994).

Consider the partially linear model

$$
\operatorname{logit}\left(p_{i t}\right)=\log \left(\frac{p_{i t}}{1-p_{i t}}\right)=\mathbf{x}_{i t}^{\prime} \boldsymbol{\beta}+m\left(z_{i t}\right),
$$

where $\boldsymbol{\beta}$ is a $p \times 1$ vector of regression parameters and $m($.$) is an unidentified smooth$ function. In this setting, the effects of $\mathbf{X}$ are modeled parametrically and those of $\mathbf{Z}$ are modeled nonparametrically. 
The marginal variance of the response variable $y_{i t}$ is stated as

$$
v_{i t}=\operatorname{var}\left(y_{i t} \mid \mathbf{x}_{\mathbf{i t}}, z_{i t}\right)=p_{i t}\left(1-p_{i t}\right)
$$

Assume that $y_{i t}$ and $y_{i^{\prime} t^{\prime}}$ are uncorrelated when $i \neq i^{\prime}$. Let

$$
\operatorname{corr}\left(y_{i t}, y_{i t^{\prime}}\right)=\alpha_{t t^{\prime}}
$$

be the correlation between $y_{i t}$ and $y_{i t^{\prime}}$ for given $\mathbf{x}_{i t}$, where $\boldsymbol{\alpha}=\left(\alpha_{12}, \ldots, \alpha_{1 T}, \alpha_{23}, \ldots\right.$, $\left.\alpha_{T-1, T}\right)^{\prime}$ is a vector of correlation parameters. If $m($.$) is specified as the identity$ function, then model (5.3) becomes an ordinary logistic regression model.

\subsubsection{Dropout Model}

Let $R_{i t}$ be an indicator variable taking the value 1 if the response $y_{i t}$ is observed and 0 otherwise. Then $\mathbf{R}_{i}=\left(R_{i 1}, R_{i 2}, \ldots, R_{i T}\right)^{\prime}$ represents a vector of response $\mathbf{y}_{i}=$

$\left(y_{i 1}, \ldots, y_{i T}\right)^{\prime}$. We assume a monotone missing data pattern, where $R_{i 1} \geq \cdots \geq R_{i T}$ and $R_{i 1}=1$ for all subjects.

In general, the dropout mechanism depends on the full vector of responses, which includes the unobserved components of $\mathbf{y}_{i}$ and the vectors of covariates $\mathbf{X}_{i}$ and $\mathbf{Z}_{i}$. Let

$$
\lambda_{i t}=P\left(R_{i t}=1 \mid R_{i 1}=\cdots=R_{i, t-1}=1, \mathbf{y}_{i}, \mathbf{X}_{i}, \mathbf{Z}_{i}, \boldsymbol{\tau}\right)
$$

be the probability that the $i^{\text {th }}$ subject is observed at time $t$, given that the subject is observed at previous $t-1$ time points and given the response vector $\mathbf{y}_{i}$ and covariates $\left(\mathbf{X}_{i}, \mathbf{Z}_{i}\right)$, where $\boldsymbol{\tau}$ is referred to as "nuisance parameters" of the missing data model. Let the observed and unobserved (missing) components of the response vector $\mathbf{y}_{i}$ is denoted by the vector $\mathbf{y}_{i}^{o}$ and $\mathbf{y}_{i}^{m}$. For MCAR (missing completely at random), we have $\lambda_{i t}=P\left(R_{i t}=1 \mid R_{i 1}, \ldots, R_{i, t-1}=1, \mathbf{X}_{i}, \mathbf{Z}_{i}, \boldsymbol{\tau}\right)$ and for MAR (missing at random), $\lambda_{i t}=P\left(R_{i t}=1 \mid R_{i 1}, \ldots, R_{i, t-1}=1, \mathbf{y}_{i}^{o}, \mathbf{X}_{i}, \mathbf{Z}_{i}, \boldsymbol{\tau}\right)$. For NI (non-ignorable) missingness, $\lambda_{i t}$ depends on the unobserved values of the response variable. We can 
estimate $\lambda_{i t}$ from a logistic regression model using the observed data.

In the case of dropouts, the random vector $\mathbf{R}_{i}=\left(R_{i 1}, \ldots, R_{i T}\right)^{\prime}$ of binary indicators can be characterized by a single random variable

$$
M_{i}=1+\sum_{t=1}^{T} R_{i t}
$$

which indicates the dropout time. In this case, the dropout or missing data process can be defined by:

$$
\nu_{m_{i}}=f_{M_{i}}\left(m_{i} \mid \mathbf{y}_{i}, \mathbf{X}_{i}, \mathbf{Z}_{i}, \boldsymbol{\tau}\right)=P\left(M_{i}=m_{i} \mid \mathbf{y}_{i}, \mathbf{X}_{i}, \mathbf{Z}_{i}, \boldsymbol{\tau}\right)
$$

Here $M_{i}$ takes values between 2 and $\mathrm{T}+1$ if all subjects are observed on the first occasion, where the maximum value $\mathrm{T}+1$ corresponds to a complete sequence of measurements. We can show that

$$
\begin{aligned}
P\left(M_{i}=m \mid \mathbf{y}_{i}, \mathbf{X}_{i}, \mathbf{Z}_{i}, \boldsymbol{\tau}\right) & =P\left(R_{i 2}=\cdots=R_{i, m-1}=1, R_{i m}=0 \mid y_{i 1}, \ldots, y_{i m}, \mathbf{X}_{i}, \mathbf{Z}_{i}, \boldsymbol{\tau}\right) \\
& =\left\{\prod_{t=2}^{m-1} P\left(R_{i t}=1 \mid R_{i 1}=\cdots=R_{i, t-1}=1, y_{i 1}, \ldots, y_{i t}, \mathbf{X}_{i}, \boldsymbol{\tau}\right)\right\} \\
& \times P\left(R_{i m}=0 \mid R_{i 1}=\cdots=R_{i m-1}=1, y_{i 1}, \ldots, y_{i m}, \mathbf{X}_{i}, \mathbf{Z}_{i}, \boldsymbol{\tau}\right)^{I\{m \leq T\}} .
\end{aligned}
$$

where $I\{\}$ is an indicator variable.

\subsubsection{Methods of Estimation}

\subsubsection{Weighted Regression Spline Generalized Estimating Equations (WRSGEEs)}

The estimating functions $\mathbf{U}_{\boldsymbol{\beta}^{*}}\left(\boldsymbol{\beta}^{*}, \boldsymbol{\alpha}, m().\right)$ and $\mathbf{U}_{\boldsymbol{\alpha}}\left(\boldsymbol{\beta}^{*}, \boldsymbol{\alpha}, m().\right)$ for a partially linear model, as defined in Chapter 4, involved an unidentified smooth function $m($.$) .$ Nonparametric techniques are used to estimate $m($.$) . The idea is to approximate$ the function $m($.$) by the regression spline (4.8).$ 
Recall that we partitioned $\mathbf{y}_{i}$ into observed and unobserved (missing) components as $\left(\mathbf{y}_{i}^{o}, \mathbf{y}_{i}^{m}\right)$. Similarly, we considered partitioning the mean vector $\mathbf{p}_{i}$ into $\left(\mathbf{p}_{i}^{o}, \mathbf{p}_{i}^{m}\right)$. Here we incorporate the inverse probability weight of Robins et al. (1995) into the corresponding generalized estimating equations and propose a weighted second-order GEE approach and regression spline smoother for the simultaneous estimation of $m(),. \boldsymbol{\beta}^{*}$ and $\boldsymbol{\alpha}$ for the binary longitudinal model with dropouts.

The estimation algorithm is described below.

Step 0. Consider initial values $\left(\hat{\boldsymbol{\beta}}_{0}^{*}, \hat{\boldsymbol{\alpha}}_{0}\right)$.

Step 1. For a given number and locations of knots, approximate $m\left(z_{i t}\right)$ by $\hat{m}\left(z_{i t}\right)=\sum_{j=0}^{l} \hat{\gamma}_{j o} z_{i t}^{j}+\sum_{r=1}^{q} \hat{\gamma}_{l+r, o}\left(z_{i t}-\zeta_{r}\right)_{+}^{l}$.

Step 2. Given the approximate $\hat{m}(z)$, and initial estimates of $\left(\hat{\boldsymbol{\beta}}_{o}^{*}, \hat{\boldsymbol{\alpha}}_{o}\right)$, updata $\left(\hat{\boldsymbol{\beta}}^{*}, \hat{\boldsymbol{\alpha}}\right)$ by solving the estimating equations

$$
\begin{gathered}
\hat{\mathbf{U}}_{\boldsymbol{\beta}^{*}}\left(\boldsymbol{\beta}^{*}, \boldsymbol{\alpha}, m(.)\right)=\sum_{i=1}^{k} \frac{1}{\nu_{i m}} \mathbf{D}_{i}^{\prime} \mathbf{V}_{i}^{-1}\left(\mathbf{y}_{i}^{o}-\mathbf{p}_{i}^{o}\left(\boldsymbol{\beta}^{*}, \boldsymbol{\alpha}\right)\right)=0, \\
\hat{\mathbf{U}}_{\boldsymbol{\alpha}}\left(\boldsymbol{\beta}^{*}, \boldsymbol{\alpha}, m(.)\right)=\sum_{i=1}^{k} \frac{1}{\nu_{i m}} \mathbf{L}_{i}^{\prime} \mathbf{W}_{i}^{-1}\left(\mathbf{r}_{i}^{o}-\boldsymbol{\delta}_{i}^{o}\right)=0
\end{gathered}
$$

where $\mathbf{p}_{i}^{o}\left(\boldsymbol{\beta}^{*}, \boldsymbol{\alpha}\right)=\left(p_{i 1}^{o}\left(\boldsymbol{\beta}^{*}, \boldsymbol{\alpha}\right), \ldots, p_{i T}^{o}\left(\boldsymbol{\beta}^{*}, \boldsymbol{\alpha}\right)\right)^{\prime}$ is the vector of observed probabilities with

$$
\operatorname{logit}\left\{p_{i t}^{o}\left(\boldsymbol{\beta}^{*}\right)\right\}=\mathbf{x}_{i t}^{\prime} \boldsymbol{\beta}+\hat{m}\left(z_{i t}\right) .
$$

Step 3. Repeat Steps 1 and $\mathbf{2}$ until convergence in $\left(\hat{\boldsymbol{\beta}}^{*}, \hat{\boldsymbol{\alpha}}\right)$.

When applying the above-mentioned procedure it is practical to select an initial value by the GEE method assuming an identity function for $m($.$) and then estimating$ the parameters based on an ordinary logistic regression model. Our computational knowledge suggests that the procedure is not rigorous, but slightly sensitive to the choice of starting values. 


\subsubsection{Estimating Dropout Probabilities}

Prior to solving the iterative equations (5.10) and (5.11), we estimate the response probability weights $\nu_{i m}$. The pseudo-likelihood function for $\boldsymbol{\tau}$ is obtained from equation (5.9) as

$$
\begin{gathered}
L(\boldsymbol{\tau})=\prod_{i=1}^{k} P\left(M_{i}=m_{i} \mid \mathbf{y}_{i t}^{*}, \boldsymbol{\tau}\right) \\
=\prod_{i=1}^{k}\left\{\prod_{t=2}^{m_{i}-1} \frac{1}{1+\exp \left(\mathbf{y}_{i t}^{*^{\prime}} \boldsymbol{\tau}\right)}\right\}\left\{\frac{\exp \left(\mathbf{y}_{i m_{i}}^{*^{\prime}} \boldsymbol{\tau}\right)}{1+\exp \left(\mathbf{y}_{i m_{i}}^{*^{\prime}} \boldsymbol{\tau}\right)}\right\}^{I\left\{m_{i} \leq T\right\}},
\end{gathered}
$$

where $\mathbf{y}_{i t}^{*}=\left(1, y_{i, t-1}, y_{i t}, \mathbf{x}_{i t}^{\prime}, z_{i t}\right)^{\prime}$ and $\boldsymbol{\tau}=\left(\tau_{0}, \tau_{1}, \tau_{2}, \ldots\right)^{\prime}$. Let

$$
\operatorname{logit}\left\{P_{i t}^{*}(\boldsymbol{\tau})\right\}=\mathbf{y}_{i t}^{*^{\prime}} \boldsymbol{\tau}
$$

The pseudo-ML estimator of $\boldsymbol{\tau}$ is obtained by maximizing the likelihood function (5.13). The pseudo-score equation for $\tau$ take the form

$$
\begin{aligned}
S_{\boldsymbol{\tau}}(\boldsymbol{\tau})=\frac{\partial \log L(\boldsymbol{\tau})}{\partial \boldsymbol{\tau}} & =\sum_{i=1}^{k} S_{\boldsymbol{\tau}, i}(\boldsymbol{\tau}) \\
& =\sum_{i=1}^{k}\left\{-\sum_{t=2}^{m_{i}-1} P_{i t}^{*}(\boldsymbol{\tau}) \mathbf{y}_{i t}^{*}+I\left\{m_{i} \leq T\right\}\left\{1-P_{i m_{i}}^{*}(\boldsymbol{\tau})\right\} \mathbf{y}_{i m_{i}}^{*}\right\}=0 .
\end{aligned}
$$

The pseudo-information matrix is obtained as

$$
I_{\boldsymbol{\tau}}(\boldsymbol{\tau})=\sum_{i=1}^{k} \sum_{t=2}^{\min \left(m_{i}, T\right)} P_{i t}^{*}(\boldsymbol{\tau})\left(1-P_{i t}^{*}(\boldsymbol{\tau})\right) \mathbf{y}_{i t}^{*} \mathbf{y}_{i t^{\prime}}^{*}
$$

The Newton-Raphson iterative procedure is applied to solve estimating equations (5.15) for the pseudo-ML estimator $\hat{\boldsymbol{\tau}}=\left(\hat{\tau}_{0}, \hat{\tau}_{1}, \hat{\tau}_{2}, \ldots\right)^{\prime}$. For the $i^{\text {th }}$ individual at time $t$, the predicted probability of response is obtained as

$$
1-\hat{P}_{i t}^{*}=\frac{1}{1+\exp \left(\mathbf{y}_{i t}^{*} \hat{\boldsymbol{\tau}}\right)}
$$


For the $i^{\text {th }}$ individual at time $m_{i}$, the dropout probability is estimated by

$$
\hat{\nu}_{i m_{i}}=P\left(M_{i}=m_{i} \mid \hat{\mathbf{y}}_{i t}^{*^{\prime}}, \hat{\boldsymbol{\tau}}\right)=\left\{\prod_{t=2}^{m_{i}-1}\left(1-\hat{P}_{i t}^{*}\right)\right\} \times\left\{\hat{P}_{i m_{i}}^{*}\right\}^{I\left\{m_{i} \leq T\right\}}
$$

Then in equations (5.10) and (5.11), we replace $\nu_{i m}$ by $\hat{\nu}_{i m}$ and iteratively solve these equations.

\subsubsection{Computational Details for Estimating the Response Model}

Recall the algorithm discussed in Section 5.2.3.1. In Step 1, we approximate the nonlinear function $m\left(z_{i t}\right)$ by

$$
\hat{m}\left(z_{i t}\right)=\Phi_{i t}^{\prime} \gamma
$$

for which the success probability $p_{i t}$ is approximated by

$$
\operatorname{logit}\left(p_{i t}\right)=\mathbf{x}_{i t}^{*^{\prime}} \boldsymbol{\beta}^{*}=\boldsymbol{x}_{i \boldsymbol{t}}^{\prime} \boldsymbol{\beta}+\Phi_{i t}^{\prime} \boldsymbol{\gamma}
$$

The iterative procedure for computing $\left(\hat{\boldsymbol{\beta}}^{*}, \hat{\boldsymbol{\alpha}}\right)$ initiates with some preliminary values $\left(\hat{\boldsymbol{\beta}}_{0}^{*}, \hat{\boldsymbol{\alpha}}_{0}\right)$ from Step $\mathbf{0}$ of our algorithm and yields updated estimates $\left(\hat{\boldsymbol{\beta}}_{s+1}^{*}, \hat{\boldsymbol{\alpha}}_{s+1}\right)$ from $\left(\hat{\boldsymbol{\beta}}_{s}^{*}, \hat{\boldsymbol{\alpha}}_{s}\right)$ by means of the iterative equations

$$
\begin{gathered}
\hat{\boldsymbol{\beta}}_{s+1}^{*}=\hat{\boldsymbol{\beta}}_{s}^{*}+\left(\sum_{i=1}^{k} \frac{1}{\nu_{i m}} \mathbf{D}_{i}^{\prime} \mathbf{V}_{i}^{-1} \mathbf{D}_{i}\right)^{-1} \sum_{i=1}^{k} \frac{1}{\nu_{i m}} \mathbf{D}_{i}^{\prime} \mathbf{V}_{i}^{-1}\left(\mathbf{y}_{i}^{o}-\mathbf{p}_{i}^{o}(\boldsymbol{\beta}, \boldsymbol{\gamma}, \boldsymbol{\alpha})\right), \\
\hat{\boldsymbol{\alpha}}_{s+1}=\hat{\boldsymbol{\alpha}}_{s}+\left(\sum_{i=1}^{k} \frac{1}{\nu_{i m}} \mathbf{L}_{i}^{\prime} \mathbf{W}_{i}^{-1} \mathbf{L}_{i}\right)^{-1} \sum_{i=1}^{k} \frac{1}{\nu_{i m}} \mathbf{L}_{i}^{\prime} \mathbf{W}_{i}^{-1}\left(\mathbf{r}_{i}^{o}-\boldsymbol{\delta}_{i}^{o}\right),
\end{gathered}
$$

for $s=0,1,2, \ldots$, where the second expression on the right side of every estimating equation is evaluated at the present estimates $\left(\hat{\boldsymbol{\beta}}_{s}^{*}, \hat{\boldsymbol{\alpha}}_{s}\right)$. 


\subsubsection{Asymptotic Properties}

\subsubsection{Asymptotic for $\hat{m}($.}

In this section, the asymptotic properties of our proposed WGEE estimators are studied. Along with assumptions A1 - A5 given in Chapter 4, some additional assumptions are needed to establish the asymptotic results in the case of missing data.

A6. The parameter vector $\boldsymbol{\tau}_{o}$ is an interior point of the parametric space $\boldsymbol{\Xi}$, which is a compact set.

A7. $\lambda_{i t}(\boldsymbol{\tau})>C_{3}>0$ for all $\boldsymbol{\tau} \in \boldsymbol{\Xi}$, for some constant $C_{3}$.

A8. $\boldsymbol{I}_{\boldsymbol{\tau}}\left(\boldsymbol{\tau}_{o}\right)=\frac{1}{n} \frac{\boldsymbol{\partial}}{\boldsymbol{\partial} \tau} S_{\boldsymbol{\tau}}\left(\boldsymbol{\tau}_{o}\right)$ and $\frac{1}{n} S_{\boldsymbol{\tau}}\left(\boldsymbol{\tau}_{o}\right) S_{\boldsymbol{\tau}}\left(\boldsymbol{\tau}_{o}\right)^{\prime}$ converges to $\boldsymbol{\Sigma}_{\boldsymbol{\tau}}$ and $\mathbf{V}_{\boldsymbol{\tau}}$, respectively, in probability for some positive definite matrix $\boldsymbol{\Sigma}_{\boldsymbol{\tau}}$ and $\mathbf{V}_{\boldsymbol{\tau}}$.

Let $\mathbf{J}_{i}\left(\mathbf{p}_{i}\left(\boldsymbol{\beta}^{*}\right), \boldsymbol{\tau}\right)=\frac{1}{\nu_{i m}}\left(\mathbf{y}_{i}^{o}-\mathbf{p}_{i}^{o}\left(\boldsymbol{\beta}^{*}\right)\right)$, where $\nu_{i m}$ represent the response probability weight of the $i^{\text {th }}$ individual. Similarly, let $\mathbf{J}_{i}\left(\mathbf{p}_{o i}, \boldsymbol{\tau}_{o}\right)=\frac{1}{\nu_{i m}}\left(\mathbf{y}_{i}^{o}-\mathbf{p}_{o i}^{o}\right)$, evaluated at true $\mathbf{p}_{o i}$ and $\boldsymbol{\tau}_{o}$.

Lemma 2. Assume assumptions A6 - A8 hold. Then

$$
\sqrt{n}\left(\hat{\tau}-\tau_{o}\right) \longrightarrow \mathbf{N}\left(\mathbf{0}, \Sigma_{\tau}^{-1} \mathbf{V}_{\tau} \Sigma_{\tau}^{-1}\right)
$$

Theorem 2. Assume that assumptions $\mathbf{A} \mathbf{1}-\mathbf{A} \mathbf{8}$ hold. If the number of knots is $q \approx n^{1 /(2 h+1)}$, then

$$
\frac{1}{n} \sum_{i=1}^{k} \sum_{t=1}^{T}\left(\hat{m}\left(z_{i t}\right)-m\left(z_{i t}\right)\right)^{2}=O_{p}\left(n^{-2 h /(2 h+1)}\right),
$$

where $\hat{m}(z)=\Pi(z)^{\prime} \hat{\boldsymbol{\Gamma}}$ and

$$
\sqrt{n}\left(\hat{\boldsymbol{\beta}}-\boldsymbol{\beta}_{o}\right) \longrightarrow \mathbf{N}\left(\mathbf{0}, \mathbf{V}_{\boldsymbol{\beta}}\right)
$$


where $\mathbf{V}_{\boldsymbol{\beta}}=\mathbf{H}_{n}^{-1} \mathbf{O}_{n} \mathbf{H}_{n}^{-1}$. The asymptotic covariance matrix of $\hat{\boldsymbol{\beta}}$ can be estimated by

$$
\mathbf{V}(\hat{\boldsymbol{\beta}})=\mathbf{H}_{\hat{\boldsymbol{\beta}}}^{-1} \mathbf{O}_{\hat{\boldsymbol{\beta}}} \mathbf{H}_{\hat{\boldsymbol{\beta}}}^{-1}
$$

where $\mathbf{H}_{\hat{\boldsymbol{\beta}}}=\sum_{i=1}^{k} \frac{1}{\nu_{i m}} \widehat{\mathbf{D}}_{i}^{\prime} \hat{\mathbf{V}}_{i}^{-1} \widehat{\mathbf{D}}_{i}$ and $\mathbf{O}_{\hat{\boldsymbol{\beta}}}=\sum_{i=1}^{k} \mathbf{S}_{\hat{\boldsymbol{\beta}}, i} \mathbf{S}_{\hat{\boldsymbol{\beta}}, i}^{\prime}$ with $\mathbf{S}_{\hat{\boldsymbol{\beta}}, i}=\frac{1}{\nu_{i m}} \widehat{\mathbf{D}}_{i}^{\prime} \hat{\mathbf{V}}_{i}^{-1}\left(\mathbf{y}_{i}^{o}-\hat{\mathbf{p}}_{i}^{o}\right)$.

For consistency, the dimension of the approximating spline space must increase with the number of subjects $k$, and the number of knots must be selected appropriately to balance between the bias and variance. Also Lemma 1 in Chapter 4 plays an important role in the proof of Theorem 2 .

We approximate $m(z)$ by $\Pi(z)^{\prime} \boldsymbol{\Gamma}$, thus we have $\eta_{i t}\left(\boldsymbol{\beta}^{*}\right)=f\left(p_{i t}\left(\boldsymbol{\beta}^{*}\right)=\mathbf{x}_{i t}^{\prime} \boldsymbol{\beta}+\Pi_{i t}^{\prime} \boldsymbol{\Gamma}\right.$, with $\boldsymbol{\beta}^{*}=\left(\boldsymbol{\beta}^{\prime}, \boldsymbol{\Gamma}^{\prime}\right)_{\left(p+N_{q}\right) \times 1}^{\prime}$.

\subsubsection{Proof of Theorem 2:}

Let

$$
\begin{aligned}
\boldsymbol{\xi}\left(\boldsymbol{\beta}^{*}\right) & =\left(\begin{array}{l}
\boldsymbol{\xi}_{1} \\
\boldsymbol{\xi}_{2}
\end{array}\right)=\left(\mathbf{T}_{n}^{\prime}\right)^{-1}\left(\boldsymbol{\beta}^{*}-\boldsymbol{\beta}_{o}^{*}\right) \\
& =\left(\begin{array}{c}
\mathbf{H}_{n}^{1 / 2}\left(\boldsymbol{\beta}-\boldsymbol{\beta}_{o}\right) \\
q^{-1 / 2} \mathbf{K}_{n}\left(\boldsymbol{\Gamma}-\boldsymbol{\Gamma}_{o}\right)+q^{1 / 2} \mathbf{K}_{n}^{-1} \mathbf{M}^{\prime} \Sigma_{o} \mathbf{X}\left(\boldsymbol{\beta}-\boldsymbol{\beta}_{o}\right)
\end{array}\right),
\end{aligned}
$$

and

$$
\hat{\xi}=\left(\begin{array}{l}
\hat{\xi_{1}} \\
\hat{\xi_{2}}
\end{array}\right)=\xi(\hat{\beta}, \hat{\Gamma})
$$

where

$$
\mathbf{T}_{n}=\left(\begin{array}{cc}
\mathbf{H}_{n}^{-1 / 2} & -\mathbf{H}_{n}^{-1 / 2} \mathbf{X}^{\prime} \boldsymbol{\Sigma}_{\mathbf{o}} \mathbf{M}\left(\mathbf{M}^{\prime} \boldsymbol{\Sigma}_{\mathbf{o}} \mathbf{M}\right)^{-\mathbf{1}} \\
0 & q^{-1 / 2} \mathbf{K}_{n}^{-1}
\end{array}\right)
$$

with $\mathbf{H}_{n}=\tilde{\mathbf{X}}^{\prime} \boldsymbol{\Sigma}_{o} \tilde{\mathbf{X}}=\sum_{i=1}^{k} \tilde{\mathbf{X}}_{i}^{\prime} \boldsymbol{\Sigma}_{o i} \tilde{\mathbf{X}}_{i}$ and $\mathbf{K}_{n}^{2}=q \mathbf{M}^{\prime} \boldsymbol{\Sigma}_{o} \mathbf{M}$. Let $\varrho_{n i}=\boldsymbol{\Pi}\left(\mathbf{Z}_{i}\right) \boldsymbol{\Gamma}_{o}-$ $m\left(\mathbf{Z}_{i}\right)$ and $\boldsymbol{\varsigma}_{i}=\overline{\mathbf{X}}_{i} \boldsymbol{\xi}_{\mathbf{1}}+\tilde{\boldsymbol{\Pi}}_{i} \boldsymbol{\xi}_{\mathbf{2}}+\boldsymbol{\varrho}_{n i}$, where $\widehat{\mathbf{X}}_{i}=\left(\overline{\mathbf{X}}_{i}, \tilde{\boldsymbol{\Pi}}_{i}\right), \overline{\mathbf{X}}_{i}^{\prime}=\mathbf{H}_{n}^{-1 / 2} \tilde{\mathbf{X}}_{i}^{\prime}$ and $\tilde{\boldsymbol{\Pi}}_{i}^{\prime}=q^{1 / 2} \mathbf{K}_{n}^{-1} \boldsymbol{\Pi}\left(\mathbf{Z}_{i}^{\prime}\right)$. Thus the regression problem $f\left(\mathbf{p}_{i}\left(\boldsymbol{\beta}^{*}\right)\right)=\boldsymbol{\eta}_{i}\left(\boldsymbol{\beta}^{*}\right)=\mathbf{X}_{i}^{*} \boldsymbol{\beta}^{*}$ becomes $\boldsymbol{\eta}_{i}(\boldsymbol{\xi})=\mathbf{X}_{i}^{*} \boldsymbol{\beta}^{*}=\boldsymbol{\eta}_{o i}+\boldsymbol{\varsigma}_{i}$, for $i=1, \ldots, k$, where $\boldsymbol{\eta}_{o i}=\mathbf{X}_{i} \boldsymbol{\beta}_{o}+m\left(\mathbf{Z}_{i}\right)$, 
and $\mathbf{p}_{i}\left(\boldsymbol{\beta}^{*}\right)=f^{-1}\left(\boldsymbol{\eta}_{o i}+\boldsymbol{\varsigma}_{i}\right)$ with $f=$ logit link function. Thus the partially linear WGEE estimating equation for $\boldsymbol{\beta}^{*}$ becomes

$$
\mathbf{U}_{\boldsymbol{\xi}}(\mathbf{p}(\boldsymbol{\xi}))=\sum_{i=1}^{k} \mathbf{D}_{i}^{\prime}\left(\mathbf{p}_{i}^{o}(\boldsymbol{\xi})\right) \mathbf{V}_{i}^{-1}\left(\mathbf{p}_{i}^{o}(\boldsymbol{\xi})\right) \mathbf{J}_{i}\left(\mathbf{p}_{i}^{o}(\boldsymbol{\xi}), \hat{\boldsymbol{\tau}}\right)=0
$$

where $\mathbf{D}_{i}\left(\mathbf{p}_{i}^{o}(\boldsymbol{\xi})\right)=\boldsymbol{\Delta}_{i}\left(\mathbf{p}_{i}^{o}(\boldsymbol{\xi})\right) \mathbf{X}_{i}$. Thus we have

$$
\begin{aligned}
\boldsymbol{\psi}(\boldsymbol{\xi})= & \left(\begin{array}{c}
\boldsymbol{\psi}_{\mathbf{1}}(\boldsymbol{\xi}) \\
\boldsymbol{\psi}_{\mathbf{2}}(\boldsymbol{\xi})
\end{array}\right)=\mathbf{T}_{n} \mathbf{U}_{\boldsymbol{\xi}}(\mathbf{p}(\boldsymbol{\xi})) \\
= & \sum_{i=1}^{k} \widehat{\mathbf{D}}_{i}^{\prime}\left(\mathbf{p}_{i}^{o}(\boldsymbol{\xi})\right) \mathbf{V}_{i}^{-1}\left(\mathbf{p}_{i}^{o}(\boldsymbol{\xi})\right) \mathbf{J}_{i}\left(\mathbf{p}_{i}^{o}(\boldsymbol{\xi}), \hat{\boldsymbol{\tau}}\right)=0 \\
= & \left(\begin{array}{c}
\sum_{i=1}^{k} \mathbf{H}_{n}^{-1 / 2} \tilde{\mathbf{X}}_{i}^{\prime} \boldsymbol{\Delta}_{i}\left(\mathbf{p}_{i}^{o}(\boldsymbol{\xi})\right) \mathbf{V}^{-1}\left(\mathbf{p}_{i}^{o}(\boldsymbol{\xi})\right) \mathbf{J}_{i}\left(\mathbf{p}_{i}^{o}(\boldsymbol{\xi}), \hat{\boldsymbol{\tau}}\right) \\
\sum_{i=1}^{k} q^{1 / 2} \mathbf{K}_{n}^{-1} \mathbf{\Pi}_{i}^{\prime} \boldsymbol{\Delta}_{i}\left(\mathbf{p}_{i}^{o}(\boldsymbol{\xi})\right) \mathbf{V}^{-1}\left(\mathbf{p}_{i}^{o}(\boldsymbol{\xi})\right) \mathbf{J}_{i}\left(\mathbf{p}_{i}^{o}(\boldsymbol{\xi}), \hat{\boldsymbol{\tau}}\right)
\end{array}\right)=0
\end{aligned}
$$

where $\widehat{\mathbf{D}}_{i}=\left(\boldsymbol{\Delta}_{i} \tilde{\mathbf{X}}_{i} \mathbf{H}_{n}^{-1 / 2} \boldsymbol{\Delta}_{i} \boldsymbol{\Pi}_{i} \mathbf{K}_{n}^{-1} q^{1 / 2}\right)^{\prime}$. It is easy to show that both equations (5.27) and (5.28) give the same root for $\boldsymbol{\xi}$ by $\mathbf{A} 4$.

We denote

$$
\boldsymbol{\Psi}(\boldsymbol{\xi})=\sum_{i=1}^{k}\left\{\widehat{\mathbf{D}}_{o i}^{\prime} \mathbf{V}_{o i}^{-1} \mathbf{J}_{o i}-\left[\sum_{i=1}^{k} \widehat{\mathbf{D}}_{o i}^{\prime} \mathbf{V}_{o i}^{-1} \frac{\boldsymbol{\partial}}{\boldsymbol{\partial} \boldsymbol{\tau}} \mathbf{J}_{o i}\right] \boldsymbol{\Sigma}_{\boldsymbol{\tau}}^{-1} S_{\boldsymbol{\tau}, i}\left(\boldsymbol{\tau}_{o}\right)\right\}+\boldsymbol{\xi}
$$

The root obtained by solving $\boldsymbol{\Psi}(\boldsymbol{\xi})=0$

$$
\tilde{\boldsymbol{\xi}}=\left(\begin{array}{c}
\tilde{\boldsymbol{\xi}}_{\mathbf{1}} \\
\tilde{\boldsymbol{\xi}}_{\mathbf{2}}
\end{array}\right)=-\sum_{i=1}^{k}\left\{\widehat{\mathbf{D}}_{o i}^{\prime} \mathbf{V}_{o i}^{-1} \mathbf{J}_{o i}-\left[\sum_{i=1}^{k} \widehat{\mathbf{D}}_{o i}^{\prime} \mathbf{V}_{o i}^{-1} \frac{\boldsymbol{\partial}}{\boldsymbol{\partial} \boldsymbol{\tau}} \mathbf{J}_{o i}\right] \boldsymbol{\Sigma}_{\boldsymbol{\tau}}^{-1} S_{\boldsymbol{\tau}, i}\left(\boldsymbol{\tau}_{o}\right)\right\}
$$

is not an estimator. In the following, we show that the difference between $\tilde{\boldsymbol{\xi}}$ and $\hat{\boldsymbol{\xi}}$ 
is small. Let $\mathbf{a} \in \mathbf{R}^{p+N_{q}}$, where $\mathbf{a}^{\prime} \mathbf{a}=\mathbf{1}$. We expand $\mathbf{a}^{\prime} \boldsymbol{\psi}(\boldsymbol{\xi})$ in a Taylor series as

$$
\begin{aligned}
\mathbf{a}^{\prime} \boldsymbol{\psi}(\mathbf{p}(\boldsymbol{\xi}), \hat{\boldsymbol{\tau}}) & =\mathbf{a}^{\prime} \boldsymbol{\psi}\left(\mathbf{p}\left(\boldsymbol{\eta}_{o}+\boldsymbol{\varsigma}\right), \hat{\boldsymbol{\tau}}\right) \\
& =\sum_{i=1}^{k} \mathbf{a}^{\prime} \widehat{\mathbf{D}}_{i}^{\prime}\left(\mathbf{p}_{i}^{o}\left(\boldsymbol{\eta}_{o i}+\boldsymbol{\varsigma}\right), \hat{\boldsymbol{\tau}}\right) \mathbf{V}_{i}^{-1}\left(\mathbf{p}_{i}^{o}\left(\boldsymbol{\eta}_{o i}+\varsigma\right), \hat{\boldsymbol{\tau}}\right) \mathbf{J}_{i}\left(\mathbf{p}_{i}^{o}\left(\boldsymbol{\eta}_{o i}+\boldsymbol{\varsigma}_{i}\right), \hat{\boldsymbol{\tau}}\right) \\
& =\sum_{i=1}^{k} \mathbf{a}^{\prime} \widehat{\mathbf{D}}_{o i}^{\prime} \mathbf{V}_{o i}^{-1} \mathbf{J}_{i}\left(\mathbf{p}_{o i}^{o}, \hat{\boldsymbol{\tau}}\right)+\sum_{i=1}^{k} \mathbf{a}^{\prime} \widehat{\mathbf{D}}_{o i} \mathbf{V}_{o i}^{-1} \frac{\partial}{\partial \mathbf{p}_{i}} \mathbf{J}_{i}\left(\mathbf{p}_{o i}, \hat{\boldsymbol{\tau}}\right) \boldsymbol{\Delta}_{o i} \boldsymbol{\varsigma}_{i} \\
& +\sum_{i=1}^{k} \boldsymbol{\varsigma}_{i}^{\prime} \frac{\boldsymbol{\partial}}{\boldsymbol{\partial} \boldsymbol{p}_{i}}\left(\mathbf{a}^{\prime} \widehat{\mathbf{D}}_{o i}^{\prime} \mathbf{V}_{o i}^{-1}\right) \mathbf{J}_{i}\left(\mathbf{p}_{o i}, \hat{\boldsymbol{\tau}}\right)+\varrho_{n}^{*}\left(\mathbf{p}^{*}, \hat{\boldsymbol{\tau}}\right) \\
& =: \mathbf{l}_{\mathbf{1}}+\mathbf{l}_{\mathbf{2}}+\mathbf{l}_{\mathbf{3}}+\mathbf{l}_{\mathbf{4}},
\end{aligned}
$$

where $\varrho_{n}^{*}\left(\mathbf{p}^{*}, \hat{\boldsymbol{\tau}}\right)=\sum_{i=1}^{k} \varrho^{*}\left(\mathbf{p}_{i}^{*}, \hat{\boldsymbol{\tau}}\right)$ with

$\varrho^{*}\left(\mathbf{p}_{i}^{*}, \hat{\boldsymbol{\tau}}\right)=\frac{1}{2} \boldsymbol{\varsigma}_{i}^{\prime} \frac{\partial^{\mathbf{2}}}{\partial \boldsymbol{p}_{i} \partial \boldsymbol{p}_{i}^{\prime}}\left(\mathbf{a}^{\prime} \widehat{\mathbf{D}}_{i} \mathbf{V}_{i}^{-1} \mathbf{J}_{i}\left(\mathbf{p}^{*}, \hat{\boldsymbol{\tau}}\right)\right) \boldsymbol{\varsigma}_{i}$, evaluated at $\mathbf{p}_{i}^{*}=\left(\mathbf{p}\left(\boldsymbol{\eta}_{o i}+\boldsymbol{\iota}_{i} \boldsymbol{\varsigma}_{i}\right)\right.$, for $1 \leq i \leq k, 0<\boldsymbol{\iota}_{i}<1$.

Consider the term $\mathbf{l}_{\mathbf{1}}$ first. Applying Taylor series expansion w.r.t to $\boldsymbol{\tau}$, we have

$$
\begin{aligned}
\mathbf{l}_{\mathbf{1}} & =\sum_{i=1}^{k} \mathbf{a}^{\prime} \widehat{\mathbf{D}}_{o i}^{\prime} \mathbf{V}_{o i}^{-1} \mathbf{J}_{o i}+\sum_{i=1}^{k} \mathbf{a}^{\prime} \widehat{\mathbf{D}}_{o i}^{\prime} \mathbf{V}_{o i}^{-1}\left(\frac{\boldsymbol{\partial}}{\boldsymbol{\partial \tau}} \mathbf{J}_{o i}\right)\left(\hat{\boldsymbol{\tau}}-\boldsymbol{\tau}_{o}\right) \\
& +\left.\frac{\mathbf{1}}{\mathbf{2}} \sum_{i=1}^{k}\left(\hat{\boldsymbol{\tau}}-\boldsymbol{\tau}_{o}\right)^{\prime} \frac{\boldsymbol{\partial}}{\boldsymbol{\partial \tau} \boldsymbol{\partial} \boldsymbol{\tau}^{\prime}}\left[\mathbf{a}^{\prime} \widehat{\mathbf{D}}_{o i}^{\prime} \mathbf{V}_{o i}^{-1} \mathbf{J}_{i}\left(\mathbf{p}_{o i}, \boldsymbol{\tau}\right)\right]\right|_{\boldsymbol{\tau}=\boldsymbol{\tau}^{*}}\left(\hat{\boldsymbol{\tau}}-\boldsymbol{\tau}_{o}\right) \\
= & : \mathbf{l}_{\mathbf{1}, \mathbf{1}}+\mathbf{l}_{\mathbf{1 , 2}}+\mathbf{l}_{\mathbf{1 , 3}},
\end{aligned}
$$

where $\boldsymbol{\tau}^{*}$ is a point between lines $\hat{\boldsymbol{\tau}}$ and $\boldsymbol{\tau}_{\boldsymbol{o}}$ and $\mathbf{J}_{o i}=\mathbf{J}_{i}\left(\mathbf{p}_{o i}, \boldsymbol{\tau}_{o}\right)$.

By assumptions A6 - A8, we can show that

$n^{1 / 2}\left(\hat{\tau}-\tau_{\boldsymbol{o}}\right)=-\boldsymbol{\Sigma}_{\boldsymbol{\tau}}^{-1}\left(n^{1 / 2} S_{\tau_{\boldsymbol{o}}}\right)+o_{p}(1)$.

Combining assumptions $\mathbf{A} \mathbf{3}$ and $\mathbf{A 4}$, we have

$$
\mathbf{l}_{\mathbf{1}, \mathbf{2}}=-\left[\sum_{i=1}^{k} \mathbf{a}^{\prime} \widehat{\mathbf{D}}_{o i}^{\prime} \mathbf{V}_{o i}^{-1} \frac{\boldsymbol{\partial}}{\partial \boldsymbol{\tau}} \mathbf{J}_{o i}\right] \boldsymbol{\Sigma}_{\boldsymbol{\tau}}{ }^{-1} \sum_{i=1}^{k} S_{\boldsymbol{\tau}, i}+o_{p}\left(q^{1 / 2}\right)
$$

Note that $\left\|\hat{\boldsymbol{\tau}}-\boldsymbol{\tau}_{\boldsymbol{o}}\right\|=O_{p}\left(n^{-1 / 2}\right)$ and further $\mathbf{l}_{\mathbf{1 , 3}}=o_{p}\left(q^{1 / 2}\right)$. Combining all these results, we obtain

$$
\mathbf{l}_{\mathbf{1}}=\sum_{i=1}^{k}\left[\mathbf{a}^{\prime} \widehat{\mathbf{D}}_{o i}^{\prime} \mathbf{V}_{o i}^{-1} \mathbf{J}_{o i}-\left\{\sum_{i=1}^{k} \mathbf{a}^{\prime} \widehat{\mathbf{D}}_{o i}^{\prime} \mathbf{V}_{o i}^{-1} \frac{\boldsymbol{\partial}}{\partial \boldsymbol{\tau}} \mathbf{J}_{o i}\right\} \boldsymbol{\Sigma}_{\boldsymbol{\tau}}{ }^{-1} S_{\boldsymbol{\tau}, i}\right]+o_{p}\left(q^{1 / 2}\right)
$$


Next consider $\mathbf{l}_{\mathbf{2}}$. Using the Taylor series expansion w.r.t. $\boldsymbol{\tau}$, we have

$$
\begin{aligned}
& \mathbf{l}_{\mathbf{2}}=\sum_{i=1}^{k} \mathbf{a}^{\prime} \widehat{\mathbf{D}}_{o i}^{\prime} \mathbf{V}_{o i}^{-1}\left(\frac{\partial}{\partial \mathbf{p}_{i}} \mathbf{J}_{o i}\right) \boldsymbol{\Delta}_{o i} \boldsymbol{\varsigma}_{i}+\left.\sum_{i=1}^{k} \frac{\boldsymbol{\partial}}{\partial \boldsymbol{\tau}}\left[\mathbf{a}^{\prime} \widehat{\mathbf{D}}_{o i}^{\prime} \mathbf{V}_{o i}^{-1} \frac{\boldsymbol{\partial}}{\partial \mathbf{p}_{i}} \mathbf{J}_{i}\left(\mathbf{p}_{o i}, \boldsymbol{\tau}\right) \boldsymbol{\Delta}_{o i}^{\prime} \boldsymbol{\varsigma}_{i}\right]\right|_{\boldsymbol{\tau}=\boldsymbol{\tau}^{*}}\left(\hat{\boldsymbol{\tau}}-\boldsymbol{\tau}_{\boldsymbol{o}}\right), \\
& =\sum_{i=1}^{k} \mathbf{a}^{\prime} \widehat{\mathbf{D}}_{o i}^{\prime} \mathbf{V}_{o i}^{-1} \frac{\partial}{\partial \mathbf{p}_{i}} \mathbf{J}_{o i} \boldsymbol{\Delta}_{o i} \boldsymbol{\varsigma}_{i}+\left.\sum_{i=1}^{k} \frac{\partial}{\partial \boldsymbol{\tau}}\left[\mathbf{a}^{\prime} \widehat{\mathbf{D}}_{o i}^{\prime} \mathbf{V}_{o i}^{-1} \frac{\partial}{\partial \mathbf{p}_{i}} \mathbf{J}_{i}\left(\mathbf{p}_{o i}, \boldsymbol{\tau}\right) \widehat{\mathbf{D}}_{o i}^{\prime} \boldsymbol{\xi}\right]\right|_{\boldsymbol{\tau}=\boldsymbol{\tau}^{*}}\left(\hat{\boldsymbol{\tau}}-\boldsymbol{\tau}_{o}\right) \\
& +\left.\sum_{i=1}^{k} \frac{\partial}{\partial \boldsymbol{\tau}}\left[\mathbf{a}^{\prime} \widehat{\mathbf{D}}_{o i}^{\prime} \mathbf{V}_{o i}^{-1} \frac{\partial}{\partial \boldsymbol{p}_{i}} \mathbf{J}_{i}\left(\mathbf{p}_{o i}, \boldsymbol{\tau}\right) \boldsymbol{\Delta}_{o i} \mathbf{R}_{n_{i}}\right]\right|_{\boldsymbol{\tau}=\boldsymbol{\tau}^{*}}\left(\hat{\boldsymbol{\tau}}-\boldsymbol{\tau}_{o}\right) \\
& =: \mathrm{l}_{\mathbf{2}, \mathbf{1}}+\mathrm{l}_{\mathbf{2 , 2}}+\mathrm{l}_{\mathbf{2 , 3}},
\end{aligned}
$$

where $\boldsymbol{\tau}^{*}$ is a point between lines $\hat{\boldsymbol{\tau}}$ and $\boldsymbol{\tau}_{\boldsymbol{o}}$. Thus using assumptions A3-A5 and the result that $\left\|\hat{\boldsymbol{\tau}}-\boldsymbol{\tau}_{\boldsymbol{o}}\right\|=O_{p}\left(n^{-1 / 2}\right)$ and Lemma 1 , it can be shown that

$$
\mathbf{l}_{\mathbf{2}, \mathbf{2}}=O_{p}\left(n^{-1 / 2} q\|\boldsymbol{\xi}\|\right)=o_{p}(\|\boldsymbol{\xi}\|)
$$

and

$$
\mathbf{l}_{2,3}=O_{p}\left(q^{1 / 2-h}\right)
$$

Therefore, we have

$$
\mathbf{l}_{\mathbf{2}}=\sum_{i=1}^{k} \mathbf{a}^{\prime} \widehat{\mathbf{D}}_{o i}^{\prime} \mathbf{V}_{o i}^{-1}\left(\frac{\boldsymbol{\partial}}{\boldsymbol{\partial} \boldsymbol{p}_{i}} \mathbf{J}_{o i}\right) \boldsymbol{\Delta}_{o i} \boldsymbol{\varsigma}_{i}+o_{p}(\|\boldsymbol{\xi}\|)+o_{p}\left(q^{1 / 2}\right)
$$

Using similar arguments, we can show that

$$
\mathbf{l}_{\mathbf{3}}=\sum_{i=1}^{k} \boldsymbol{\varsigma}_{i}^{\prime} \frac{\boldsymbol{\partial}}{\boldsymbol{\partial} \boldsymbol{p}_{i}}\left(\mathbf{a}^{\prime} \widehat{\mathbf{D}}_{o i}^{\prime} \mathbf{V}_{o i}^{-1}\right) \mathbf{J}_{o i}+o_{p}(\|\boldsymbol{\xi}\|)+o_{p}\left(q^{1 / 2}\right)
$$

and

$$
\mathbf{l}_{\mathbf{4}}=\mathbf{R}_{n}^{*}\left(\mathbf{p}^{*}, \boldsymbol{\tau}_{o}\right)+o_{p}(\|\boldsymbol{\xi}\|)+o_{p}\left(q^{1 / 2}\right) .
$$


Combining $\mathbf{l}_{\mathbf{1}}, \mathbf{l}_{\mathbf{2}}, \mathbf{l}_{\mathbf{3}}$ and $\mathbf{l}_{\mathbf{4}}$, we have

$$
\begin{aligned}
& \mathbf{a}^{\prime}(\psi \mathbf{p}(\boldsymbol{\xi}), \hat{\boldsymbol{\tau}})=\sum_{i=1}^{k}\left\{\mathbf{a}^{\prime} \widehat{\mathbf{D}}_{o i}^{\prime} \mathbf{V}_{o i}^{-1} \mathbf{J}_{o i}-\right. {\left.\left[\sum_{i=1}^{k} \mathbf{a}^{\prime} \widehat{\mathbf{D}}_{o i}^{\prime} \mathbf{V}_{o i}^{-1} \frac{\boldsymbol{\partial}}{\boldsymbol{\partial} \boldsymbol{\tau}} \mathbf{J}_{o i}\right] \boldsymbol{\Sigma}_{\boldsymbol{\tau}}^{-1} \mathbf{S}_{\boldsymbol{\tau}, i}\right\} } \\
&+\sum_{i=1}^{k} \mathbf{a}^{\prime} \widehat{\mathbf{D}}_{o i}^{\prime} \mathbf{V}_{o i}^{-1} \frac{\boldsymbol{\partial}}{\boldsymbol{\partial} \mathbf{p}_{i}} \mathbf{J}_{o i} \Delta_{o i} a_{i}+\sum_{i=1}^{k} \boldsymbol{\varsigma}_{i}^{\prime} \frac{\boldsymbol{\partial}}{\boldsymbol{\partial} \mathbf{p}_{i}}\left(\mathbf{a}^{\prime} \widehat{\mathbf{D}}_{o i}^{\prime} \mathbf{V}_{o i}^{-1}\right) \mathbf{J}_{o i} \\
&+\mathbf{R}_{n}^{*}\left(\mathbf{p}^{*}, \boldsymbol{\tau}_{o}\right)+o_{p}(\|\boldsymbol{\xi}\|)+o_{p}\left(q^{1 / 2}\right)
\end{aligned}
$$

Thus the difference between $\mathbf{a}^{\prime} \boldsymbol{\psi}(\boldsymbol{\xi})$ and $\mathbf{a}^{\prime} \boldsymbol{\Psi}(\boldsymbol{\xi})$ can be expressed as

$$
\begin{aligned}
\mathbf{a}^{\prime}(\boldsymbol{\psi}(\boldsymbol{\xi})-\boldsymbol{\Psi}(\boldsymbol{\xi})) & =\sum_{i=1}^{k} \mathbf{a}^{\prime} \widehat{\mathbf{D}}_{o i}^{\prime} \mathbf{V}_{o i}^{-1} \frac{\partial}{\partial \mathbf{p}_{i}} \mathbf{J}_{o i} \boldsymbol{\Delta}_{o i} \varrho_{n i} \\
& +\sum_{i=1}^{k} \varsigma_{i}^{\prime} \frac{\partial}{\partial \mathbf{p}_{\boldsymbol{i}}}\left(\mathbf{a}^{\prime} \widehat{\mathbf{D}}_{0 i}^{\prime} \mathbf{V}_{o i}^{-1}\right) \mathbf{J}_{o i}+\mathbf{R}_{n}^{*}\left(\mathbf{p}^{*}, \boldsymbol{\tau}_{\boldsymbol{o}}\right)+o_{p}(\|\boldsymbol{\xi}\|)+o_{p}\left(q^{1 / 2}\right)
\end{aligned}
$$

Then using similar arguments as used in the proof of Theorem 1 in Chapter 4, it can be shown that

$$
\sup _{\|\boldsymbol{\xi}\| \leq L q^{1 / 2}}\|\boldsymbol{\psi}(\boldsymbol{\xi})-\boldsymbol{\Psi}(\boldsymbol{\xi})\|=O_{p}\left(q^{1 / 2}\right)
$$

for sufficiently large $L$, and for sufficiently large $C_{3}$, direct calculations give

$$
\mathbf{E}\|\tilde{\boldsymbol{\xi}}\|^{2}=O(q)
$$

Therefore,

$$
\sup _{\|\boldsymbol{\xi}\| \leq L q^{1 / 2}}\|\boldsymbol{\psi}(\boldsymbol{\xi})-\boldsymbol{\xi}\| \leq \sup _{\|\boldsymbol{\xi}\| \leq L q^{1 / 2}}\|\boldsymbol{\psi}(\boldsymbol{\xi})-\boldsymbol{\Psi}(\boldsymbol{\xi})\|+\|\tilde{\boldsymbol{\xi}}\|=L O_{p}\left(q^{1 / 2}\right)+O_{p}\left(q^{1 / 2}\right)
$$

which shows that for sufficiently large $L, \sup _{\|\boldsymbol{\xi}\| \leq L q^{1 / 2}}\|\boldsymbol{\psi}(\boldsymbol{\xi})-\boldsymbol{\xi}\| \leq L q^{1 / 2}$ in probability. Thus the theorem of Brouwer's fixed point ensures that there exist a zero $\hat{\boldsymbol{\xi}}$ of $\boldsymbol{\psi}(\boldsymbol{\xi})$ with $\|\hat{\boldsymbol{\xi}}\|=O_{p}\left(q^{1 / 2}\right)$, so that the optimum merging rate of nonparametric estimator can be attained and, by using the CLT on $\tilde{\boldsymbol{\xi}}_{\mathbf{1}}$, it is possible to establish the asymptotic normality of the estimator $\hat{\boldsymbol{\beta}}$. 


\subsubsection{Simulation Study}

To assess the performance of the proposed estimators, we ran a series of simulations using incomplete binary longitudinal data. The following three methods were compared in the simulation study.

1. GEE: Ordinary GEE approach of Prentice (1988) is used to find the estimates of $\boldsymbol{\beta}, \boldsymbol{\alpha}$, and $m($.$) . Here m($.$) is considered as a linear function.$

2. RSGEE: The unweighted regression spline GEE method is used to estimate $\boldsymbol{\beta}, \boldsymbol{\alpha}$ and unknown smooth function $m($.$) .$

3. WRSGEE: The proposed weighted regression spline GEE method is used to estimate $\boldsymbol{\beta}, \boldsymbol{\alpha}$ and unknown smooth function $m($.$) . To explore and compare the$ empirical properties of the above three methods, the following simulation set-up is used.

\subsubsection{Response Models}

To simulate the binary response for different time points, we use the following marginal model

$$
\operatorname{logit}\left[E\left(y_{i t} \mid \mathbf{x}_{i t}, z_{i t}, \boldsymbol{\beta}, \alpha\right)\right]=\beta_{1} x_{i}+\beta_{2} t+m\left(z_{i t}\right),
$$

where $m\left(z_{i t}\right)$ is a nonlinear function. The variable $x_{i}$ is a treatment indicator that takes the value 1 for $20 \%$ of the subjects and 0 for the remaining $80 \%$, thus $P\left(X_{i}=\right.$ $1)=0.2$. Marginal models are considered to be functions of time t, $x_{i}$ and $z_{i t}$, where $z_{i t}$ is generated from the uniform distribution $U(0.1,2)$. The responses from a given subject are assumed to be correlated with two types of correlation structures: exchangeable correlation $\operatorname{corr}\left(y_{i t}, y_{i t^{\prime}}\right)=\alpha$ and serial correlation $\operatorname{corr}\left(y_{i t}, y_{i t^{\prime}}\right)=$ $\alpha^{\left|t-t^{\prime}\right|}$.

The longitudinal data are obtained using a multivariate binary model as sug- 
gested by Bahadur (1961). The true values of parameters are $\beta_{1}=1, \beta_{2}=0.2$, and $\alpha=0.1$ and 0.3. We also choose $m()=.\beta_{0}+(2 * z /(0.02+z))$ with $\beta_{0}=-1$. In our proposed method, we use a linear spline function with 4 knots for approximating the nonlinear function $m($.$) . Figure 5.1$ exhibits the function $m($.$) , as used in our$ simulation study.

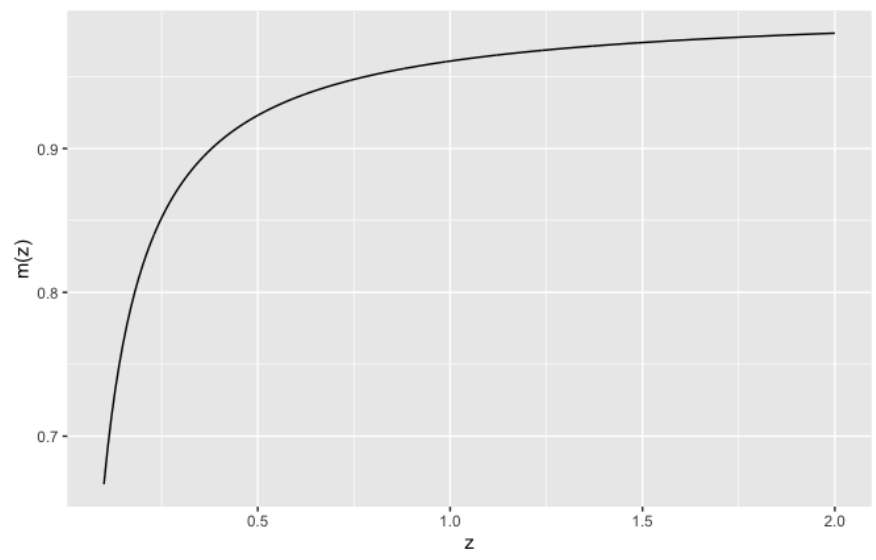

Figure 5.1: Nonlinear function $m($.

\subsubsection{Dropout Model}

We assume a MAR mechanism for the dropout probability, given by

$$
P\left(M_{i}=m_{i} \mid \mathbf{y}_{i}, \mathbf{X}_{i}, z_{i}, \boldsymbol{\tau}\right)=P\left(M_{i}=m_{i} \mid \mathbf{y}_{i}, \boldsymbol{\tau}\right)
$$

Furthermore, we assume that all subjects are measured at the first time point. We denote the response indicator with $R_{i t}$, where $R_{i t}=1$ when $y_{i t}$ is observed and 0 when missing. The probability of non-response at time $t$ is given by

$$
P\left(R_{i t}=0 \mid R_{i 1}=\cdots=R_{i t-1}=1, y_{i 1}, \ldots, y_{i t-1}, \boldsymbol{\tau}\right)=\frac{\exp \left(\psi_{i t}\right)}{1+\exp \left(\psi_{i t}\right)}
$$

where $\psi_{i t}=\tau_{0}+\tau_{1} y_{i t-1}$ for $t=2,3$. The dropout probability is given by

$$
\nu_{i m_{i}}=P\left(M_{i}=m_{i} \mid y_{i 1}, \ldots, y_{i m_{i}}, \boldsymbol{\tau}\right)=\left\{\prod_{t=2}^{m_{i}-1} \frac{1}{1+\exp \left(\psi_{i t}\right)}\right\}\left\{\frac{\exp \left(\psi_{i m_{i}}\right)}{1+\exp \left(\psi_{i m_{i}}\right)}\right\}^{I\left\{m_{i} \leq T\right\}}
$$


where $\psi_{i t}=\tau_{0}+\tau_{1} y_{i, t-1}$ and $\boldsymbol{\tau}=(-2,2)$. This choice of $\boldsymbol{\tau}$ gives approximately $35 \%$ missing values at the second and third waves. The simulation results are obtained for $S=1000$ replications and for two sample sizes $k=500$ and $k=1000$.

\subsubsection{Results}

Tables 5.1 and 5.2 present empirical biases and mean squared errors (MSEs) of the GEE, RSGEE and WRSGEE estimators of the regression parameters $\left(\beta_{1}, \beta_{2}\right)$, correlation parameter $\alpha$ and nonlinear function $m(z)$ at different values of $z=$ $(0.25,0.55,0.85,1.25,1.55)$, and $\alpha=0.1,0.3$.

It is evident from Tables 5.1 and 5.2 that the proposed WRSGEE method generally provides unbiased estimates of the model parameters under all scenarios considered in simulations. As expected, the ordinary GEE method provides large systematic biases, specifically for the estimates of the time coefficient $\beta_{2}$ and nonlinear function $m(z)$. For example, Table 5.1 shows that when estimating $\beta_{2}$ under the exchangeable correlation with $k=500$, the GEE method provides a percentage relative bias of $(0.0366 / 0.2) 100=18.3 \%$, whereas the proposed WRSGEE method provides a smaller percentage relative bias of $(0.0029 / 0.2) 100=1.45 \%$. The gain in efficiency from the WRSGEE method is more evident under a stronger correlation. For example, at $\alpha=0.3$

Table 5.2 shows that when estimating $\beta_{2}$ under the exchangeable correlation with $k=500$, the GEE method provides a large relative bias of $(0.1134 / 0.2) 100=$ $56.7 \%$, whereas the proposed WRSGEE method provides a smaller relative bias of $(0.0089 / 0.2) 100=4.45 \%$. Although the RSGEE method performs slightly better than the GEE method, but our proposed WRSGEE method appears to be the best among the three method considered here. 
Table 5.1: Empirical biases and mean squared errors (MSEs) of GEE, RSGEE and WRSGEE estimators of regression and association parameters. Correlation parameter $\alpha=0.1$.

\begin{tabular}{|c|c|c|c|c|c|c|c|}
\hline \multicolumn{8}{|c|}{ Exchangeable Corr with $\boldsymbol{\tau}=(-2,2), \mathbf{k}=\mathbf{5 0 0}$} \\
\hline \multirow[b]{2}{*}{ Parameter } & \multirow[b]{2}{*}{ True value } & \multicolumn{2}{|c|}{ GEE } & \multicolumn{2}{|c|}{ RSGEE } & \multicolumn{2}{|c|}{ WRSGEE } \\
\hline & & Bias & MSE & Bias & MSE & Bias & MSE \\
\hline$\alpha$ & 0.1 & 0.0476 & 0.0068 & 0.0485 & 0.0070 & 0.0000 & 0.0060 \\
\hline$\beta_{1}$ & 1.0 & 0.0326 & 0.0940 & 0.0358 & 0.0947 & 0.0516 & 0.1588 \\
\hline$\beta_{2}$ & 0.2 & 0.0366 & 0.0145 & 0.0376 & 0.0147 & 0.0029 & 0.0164 \\
\hline$m\left(z_{1}\right)$ & 0.85 & -0.0102 & 0.0531 & -0.0504 & 0.0766 & 0.0067 & 0.1065 \\
\hline$m\left(z_{2}\right)$ & 0.92 & -0.0627 & 0.0484 & -0.0176 & 0.0700 & 0.0277 & 0.1012 \\
\hline$m\left(z_{3}\right)$ & 0.95 & -0.0613 & 0.0442 & -0.0207 & 0.0875 & 0.0260 & 0.1323 \\
\hline$m\left(z_{4}\right)$ & 0.96 & -0.0418 & 0.0434 & -0.0323 & 0.0940 & 0.0112 & 0.1405 \\
\hline$m\left(z_{5}\right)$ & 0.97 & -0.0223 & 0.0481 & -0.0305 & 0.0757 & 0.0127 & 0.1068 \\
\hline \multicolumn{8}{|c|}{ Serial Corr with $\boldsymbol{\tau}=(-2,2), \mathbf{k}=\mathbf{5 0 0}$} \\
\hline & & \multicolumn{2}{|c|}{ GEE } & \multicolumn{2}{|c|}{ RSGEE } & \multicolumn{2}{|c|}{ WRSGEE } \\
\hline Parameter & True value & Bias & MSE & Bias & MSE & Bias & MSE \\
\hline$\alpha$ & 0.1 & 0.0567 & 0.0094 & 0.0571 & 0.0096 & 0.0035 & 0.0036 \\
\hline$\beta_{1}$ & 1.0 & 0.0444 & 0.0893 & 0.0479 & 0.0909 & 0.0721 & 0.1527 \\
\hline$\beta_{2}$ & 0.2 & 0.0362 & 0.0164 & 0.0364 & 0.0164 & 0.0108 & 0.0185 \\
\hline$m\left(z_{1}\right)$ & 0.85 & -0.0127 & 0.0543 & -0.0447 & 0.0723 & -0.0068 & 0.1025 \\
\hline$m\left(z_{2}\right)$ & 0.92 & -0.0641 & 0.0495 & -0.0236 & 0.0744 & 0.0018 & 0.1153 \\
\hline$m\left(z_{3}\right)$ & 0.95 & -0.0618 & 0.0446 & -0.0312 & 0.0970 & -0.0031 & 0.1485 \\
\hline$m\left(z_{4}\right)$ & 0.96 & -0.0409 & 0.0430 & -0.0248 & 0.0956 & 0.0048 & 0.1450 \\
\hline$m\left(z_{5}\right)$ & 0.97 & -0.0204 & 0.0471 & -0.0225 & 0.0751 & 0.0079 & 0.1054 \\
\hline \multicolumn{8}{|c|}{ Exchangeable Corr with $\boldsymbol{\tau}=(-2,2), \mathbf{k}=1000$} \\
\hline & & \multicolumn{2}{|c|}{ GEE } & \multicolumn{2}{|c|}{ RSGEE } & \multicolumn{2}{|c|}{ WRSGEE } \\
\hline Parameter & True value & Bias & MSE & Bias & MSE & Bias & MSE \\
\hline$\alpha$ & 0.1 & 0.0497 & 0.0047 & 0.0504 & 0.0048 & 0.0018 & 0.0013 \\
\hline$\beta_{1}$ & 1.0 & 0.0159 & 0.0418 & 0.0177 & 0.0418 & 0.0331 & 0.0739 \\
\hline$\beta_{2}$ & 0.2 & 0.0384 & 0.0086 & 0.0391 & 0.0087 & 0.0057 & 0.0089 \\
\hline$m\left(z_{1}\right)$ & 0.85 & -0.0057 & 0.0265 & -0.0601 & 0.0379 & -0.0146 & 0.0514 \\
\hline$m\left(z_{2}\right)$ & 0.92 & -0.0651 & 0.0268 & -0.0203 & 0.0344 & 0.0175 & 0.0504 \\
\hline$m\left(z_{3}\right)$ & 0.95 & -0.0665 & 0.0252 & -0.0395 & 0.0475 & -0.0111 & 0.0693 \\
\hline$m\left(z_{4}\right)$ & 0.96 & -0.0506 & 0.0241 & -0.0271 & 0.0449 & 0.0080 & 0.0677 \\
\hline$m\left(z_{5}\right)$ & 0.97 & -0.0338 & 0.0257 & -0.0355 & 0.0388 & 0.0032 & 0.0535 \\
\hline \multicolumn{8}{|c|}{ Serial Corr with $\boldsymbol{\tau}=(-2,2), \mathbf{k}=\mathbf{1 0 0 0}$} \\
\hline & & \multicolumn{2}{|c|}{ GEE } & \multicolumn{2}{|c|}{ RSGEE } & \multicolumn{2}{|c|}{ WRSGEE } \\
\hline Parameter & True value & Bias & MSE & Bias & MSE & Bias & MSE \\
\hline$\alpha$ & 0.1 & 0.0270 & 0.0075 & 0.0532 & 0.0059 & 0.0005 & 0.0017 \\
\hline$\beta_{1}$ & 1.0 & 0.0105 & 0.0441 & 0.0124 & 0.0442 & 0.0276 & 0.0705 \\
\hline$\beta_{2}$ & 0.2 & 0.0163 & 0.0436 & 0.0276 & 0.0076 & 0.0035 & 0.0089 \\
\hline$m\left(z_{1}\right)$ & 0.85 & 0.0256 & 0.0297 & -0.0203 & 0.0373 & -0.0107 & 0.0530 \\
\hline$m\left(z_{2}\right)$ & 0.92 & -0.0522 & 0.0263 & -0.0462 & 0.0375 & 0.0184 & 0.0523 \\
\hline$m\left(z_{3}\right)$ & 0.95 & -0.0516 & 0.0233 & -0.0227 & 0.0461 & -0.0094 & 0.0674 \\
\hline$m\left(z_{4}\right)$ & 0.96 & -0.0329 & 0.0213 & -0.0117 & 0.0467 & 0.0130 & 0.0720 \\
\hline$m\left(z_{5}\right)$ & 0.97 & -0.0141 & 0.0227 & -0.0183 & 0.0363 & 0.0062 & 0.0533 \\
\hline
\end{tabular}


Table 5.2: Empirical biases and mean squared errors (MSEs) of GEE, RSGEE and WRSGEE estimators of regression and association parameters. Correlation parameter $\alpha=0.3$.

\begin{tabular}{|c|c|c|c|c|c|c|c|}
\hline \multicolumn{8}{|c|}{ Exchangeable Corr with $\boldsymbol{\tau}=(-2,2), \mathbf{k}=\mathbf{5 0 0}$} \\
\hline \multirow[b]{2}{*}{ Parameter } & \multirow[b]{2}{*}{ True value } & \multicolumn{2}{|c|}{ GEE } & \multicolumn{2}{|c|}{ RSGEE } & \multicolumn{2}{|c|}{ WRSGEE } \\
\hline & & Bias & MSE & Bias & MSE & Bias & MSE \\
\hline$\alpha$ & 0.3 & 0.1656 & 0.0366 & 0.1690 & 0.0380 & 0.0056 & 0.0034 \\
\hline$\beta_{1}$ & 1.0 & 0.0324 & 0.1162 & 0.0365 & 0.1331 & 0.0674 & 0.2147 \\
\hline$\beta_{2}$ & 0.2 & 0.1134 & 0.0269 & 0.1142 & 0.0272 & 0.0089 & 0.0140 \\
\hline$m\left(z_{1}\right)$ & 0.85 & -0.0879 & 0.0604 & -0.1339 & 0.0820 & -0.0174 & 0.0878 \\
\hline$m\left(z_{2}\right)$ & 0.92 & -0.1398 & 0.0630 & -0.0903 & 0.0800 & 0.0221 & 0.0938 \\
\hline$m\left(z_{3}\right)$ & 0.95 & -0.1380 & 0.0572 & -0.1106 & 0.0970 & -0.0097 & 0.1143 \\
\hline$m\left(z_{4}\right)$ & 0.96 & -0.1178 & 0.0513 & -0.0912 & 0.1007 & 0.0326 & 0.1287 \\
\hline$m\left(z_{5}\right)$ & 0.97 & -0.0978 & 0.0512 & -0.1057 & 0.0826 & 0.0099 & 0.0986 \\
\hline \multicolumn{8}{|c|}{ Serial Corr with $\boldsymbol{\tau}=(-2,2), \mathbf{k}=\mathbf{5 0 0}$} \\
\hline & & \multicolumn{2}{|c|}{ GEE } & \multicolumn{2}{|c|}{ RSGEE } & \multicolumn{2}{|c|}{ WRSGEE } \\
\hline Parameter & True value & Bias & MSE & Bias & MSE & Bias & MSE \\
\hline$\alpha$ & 0.3 & 0.1735 & 0.0394 & 0.1750 & 0.0398 & 0.0103 & 0.0036 \\
\hline$\beta_{1}$ & 1.0 & 0.0267 & 0.1009 & 0.0292 & 0.1013 & 0.0639 & 0.1903 \\
\hline$\beta_{2}$ & 0.2 & 0.1276 & 0.0326 & 0.1293 & 0.0333 & 0.0223 & 0.0197 \\
\hline$m\left(z_{1}\right)$ & 0.85 & -0.1030 & 0.0665 & -0.1488 & 0.0930 & -0.0262 & 0.1071 \\
\hline$m\left(z_{2}\right)$ & 0.92 & -0.1567 & 0.0719 & -0.1139 & 0.0864 & -0.0020 & 0.1109 \\
\hline$m\left(z_{3}\right)$ & 0.95 & -0.1567 & 0.0673 & -0.1208 & 0.1036 & -0.0031 & 0.1344 \\
\hline$m\left(z_{4}\right)$ & 0.96 & -0.1388 & 0.06207 & -0.1214 & 0.11307 & -0.0135 & 0.1331 \\
\hline$m\left(z_{5}\right)$ & 0.97 & -0.1206 & 0.0620 & -0.1284 & 0.0913 & -0.0205 & 0.1066 \\
\hline \multicolumn{8}{|c|}{ Exchangeable Corr with $\boldsymbol{\tau}=(-2,2), \mathbf{k}=\mathbf{1 0 0 0}$} \\
\hline & & \multicolumn{2}{|c|}{ GEE } & \multicolumn{2}{|c|}{ RSGEE } & \multicolumn{2}{|c|}{ WRSGEE } \\
\hline Parameter & True value & Bias & MSE & Bias & MSE & Bias & MSE \\
\hline$\alpha$ & 0.3 & 0.1655 & 0.0315 & 0.1670 & 0.0319 & 0.0051 & 0.0016 \\
\hline$\beta_{1}$ & 1.0 & 0.0216 & 0.0484 & 0.0235 & 0.0487 & 0.0338 & 0.0961 \\
\hline$\beta_{2}$ & 0.2 & 0.1135 & 0.0197 & 0.1143 & 0.0199 & 0.0080 & 0.0067 \\
\hline$m\left(z_{1}\right)$ & 0.85 & -0.0914 & 0.0344 & -0.1427 & 0.0528 & -0.0258 & 0.0448 \\
\hline$m\left(z_{2}\right)$ & 0.92 & -0.1446 & 0.0426 & -0.0974 & 0.0451 & 0.0125 & 0.0466 \\
\hline$m\left(z_{3}\right)$ & 0.95 & -0.1441 & 0.0399 & -0.1088 & 0.0554 & 0.0102 & 0.0568 \\
\hline$m\left(z_{4}\right)$ & 0.96 & -0.1256 & 0.0343 & -0.1168 & 0.0568 & -0.0077 & 0.0640 \\
\hline$m\left(z_{5}\right)$ & 0.97 & -0.1069 & 0.0317 & -0.1152 & 0.0468 & -0.0083 & 0.0469 \\
\hline \multicolumn{8}{|c|}{ Serial Corr with $\boldsymbol{\tau}=(-2,2), \mathbf{k}=\mathbf{1 0 0 0}$} \\
\hline & & \multicolumn{2}{|c|}{ GEE } & \multicolumn{2}{|c|}{ RSGEE } & \multicolumn{2}{|c|}{ WRSGEE } \\
\hline Parameter & True value & Bias & MSE & Bias & MSE & Bias & MSE \\
\hline$\alpha$ & 0.3 & 0.1683 & 0.0324 & 0.1693 & 0.0327 & 0.0040 & 0.0016 \\
\hline$\beta_{1}$ & 1.0 & 0.0247 & 0.0504 & 0.0260 & 0.0508 & 0.0262 & 0.0836 \\
\hline$\beta_{2}$ & 0.2 & 0.1179 & 0.0221 & 0.1185 & 0.0222 & 0.0046 & 0.0090 \\
\hline$m\left(z_{1}\right)$ & 0.85 & -0.0930 & 0.0353 & -0.1372 & 0.0516 & -0.0186 & 0.0469 \\
\hline$m\left(z_{2}\right)$ & 0.92 & -0.1466 & 0.0439 & -0.1059 & 0.0457 & 0.0081 & 0.0502 \\
\hline$m\left(z_{3}\right)$ & 0.95 & -0.1463 & 0.0418 & -0.1206 & 0.0600 & -0.0105 & 0.0634 \\
\hline$m\left(z_{4}\right)$ & 0.96 & -0.1283 & 0.0374 & -0.1119 & 0.0546 & 0.0099 & 0.0674 \\
\hline$m\left(z_{5}\right)$ & 0.97 & -0.1098 & 0.0358 & -0.1124 & 0.0509 & 0.0095 & 0.0517 \\
\hline
\end{tabular}




\subsubsection{Application: Analysis of Smoking Data}

Here we consider a data set on trends of cigarette smoking from the Coronary Artery Development in Young Adults (CARDIA) study, where the goal was to assess the cardiovascular risk factors among white and black females and males (Hughes et al., 1987). This research was held in four metropolitan cities of the United States (Oakland, CA; Chicago, IL; Birmingham, AL, and Minneapolis, MN), where an aggregate of 5115 young grownups aged 18-30 years was prospectively followed and observed from 1986 to 1996. Recruitment, limited to white and black, was held to attain an estimated balance in size of a sample concerning gender, age, education, and race. The participants of this study were scheduled for appointments at 0,2 , 5, 7 and 10 years. We consider the initial four appointments for a total of 5078 (99.3\%) young grownups with a self-assessment status of smoking i.e., (yes/no) at known baseline (0 year). Data from following visits obtained after an individual's first missed visit were neglected to generate a set of data with monotone missingness. In particular, from a total of 17995 responses, only 578 were neglected to generate a monotone set of missing data.

The aim is to identify factors that affect the trends of cigarette smoking in young adults using a binary logistic model. The model parameters were estimated and compared using the ordinary second-order GEE approach of Prentice (1988), the unweighted semiparametric partially linear method (RSGEE ), and the proposed weighted semiparametric partially linear method (WRSGEE).

Let $y_{i t}$ be a binary response variable, which takes the value 1 if the $i^{\text {th }}$ subject at the $t^{t h}$ visit is smoker, and 0 otherwise. The marginal mean response $p_{i t}=E\left(y_{i t}\right)$ is assume to be related to the covariates by the partially linear model

$\operatorname{logit}\left(p_{i t}\right)=\beta_{0}+\beta_{1} x_{t}+\beta_{2} e_{\text {edu }}+\beta_{3}$ educ $_{i}+\beta_{4}$ raceb $_{i}+\beta_{5}$ racewm $_{i}+\beta_{6}$ racew $_{i}+m(\text { age } / 10)_{i}$

for $i=1, \ldots, 5078$ and $t=1, \ldots, 4$, where $a g e_{i}=$ age of $i^{t h}$ individual in years at baseline time; $x_{t}=$ year since the measurements of baseline $=0,2,5$ and 7 . The 
binary indicator $e d u h_{i}=1$ if level of education of $i^{t h}$ individual is high school or less, and otherwise 0 ; if level of education is up to some college, then the binary indicator $e d u c_{i}=1$, otherwise 0 . If the $i^{\text {th }}$ individual is white female, then racew $f_{i}=1$, otherwise 0 ; if the individual is black female, then $\operatorname{raceb}_{i}=1$, otherwise 0 ; and if the $i^{t h}$ individual is white male then racewm $_{i}=1$, otherwise 0 . The function $m($.$) is$ a nonlinear smooth function. An exchangeable correlation structure is considered in our proposed method. For the estimation of parameters in (5.38) by the technique of WRSGEE, first, the inverse probability weights are estimated based on the missing data model

$$
\operatorname{logit}\left(p_{i t}^{*}\right)=\tau_{0}+\tau_{1} y_{i, t-1}+\tau_{2} \text { racebf }_{i}+\tau_{3} \text { racewm }_{i}+\tau_{4} \text { racew }_{i},
$$

where $p_{i t}^{*}=P\left(R_{i t}=0 \mid R_{i 1}=\ldots, R_{i, t-1}=1, \mathbf{y}_{i}, \mathbf{X}_{i}, \boldsymbol{\tau}\right)$ is the conditional probability that at time $t$ the $i^{t h}$ individual drops out. The nonlinear function $m($ age/10) in (5.38) is approximated by the linear spline regression with 6 internal knots

$$
s(z, \gamma)=\gamma_{1}+\gamma_{2} z+\sum_{r=2}^{7} \gamma_{r+1}\left(z-\zeta_{r}\right)_{+},
$$

where $\gamma=\left(\gamma_{3}, \gamma_{4}, \ldots, \gamma_{8}\right)^{\prime}$ are the spline basis coefficients.

The pseudo-maximum likelihood estimates of missing data model parameters $\boldsymbol{\tau}=\left(\tau_{0}, \tau_{1}, \tau_{2}, \tau_{3}, \tau_{4}\right)^{\prime}$, z-values and their standard errors are presented in Table 5.3, while Table 5.4 contains the GEE, RSGEE and WRSGEE estimates of the regression parameters, their standard error, and the corresponding z-values.

From Table 5.3, it is clear that the dropout probabilities differ across gender and race as well as an individual status of smoking at the previous appointment. Young grownups have $\exp (0.389)=1.47$ times greater odds to miss an appointment in case of smoker (vs. nonsmoker) at the previous appointment. The black males are more likely to miss an appointment as compared to other gender-race arrangements. In the CARDIA study, the follow-up rates for black males were $62 \%$, for black females $68 \%$, for white males $81 \%$ and for white females $79 \%$. The fitted missing data model 
seems to reflect this situation.

It is clear from Table 5.4 that for the linear part, the WRSGEE estimates are different from the ordinary GEE and RSGEE estimates. Here the WRSGEE method based on the inverse probability weights is more reliable in the sense that it take the missingness mechanism into account when estimating the model parameters. It appears that the smoking trend is higher among young adults with a college degree or less, as compared to those with a higher degree. The smoking trend is lower among black females as compared to black males. For example, black females with high school degree or less have $\exp (1.7475)=5.7$ times higher odds of smoking as compared to those with a university degree. Also, young black females have $\exp (-0.2746)=0.76$ times lower odds of smoking as compared to young black males. The correlation coefficient $\alpha$ appears to be significant by all methods. The trend of smoking decreases over time.

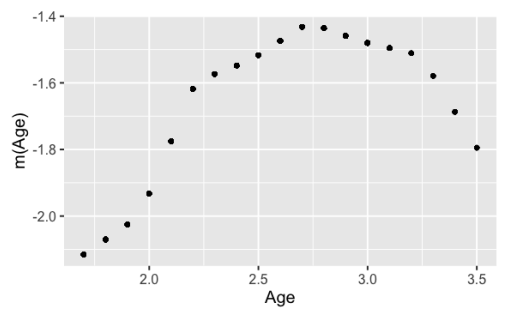

(a) WRSGEE

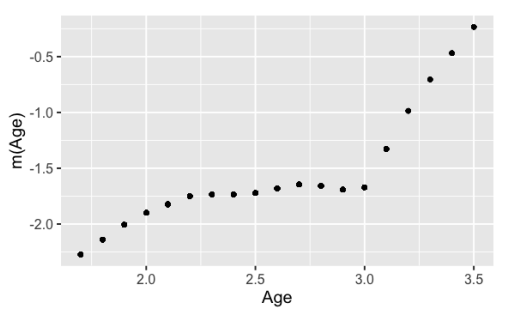

(b) RSGEE

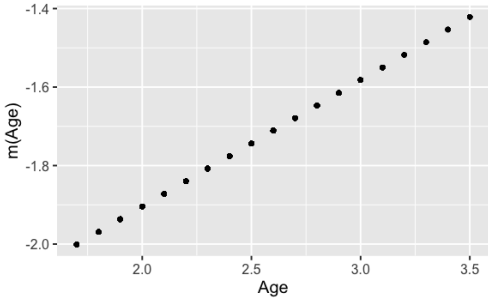

(c) GEE

Figure 5.2: Estimated nonlinear function $\hat{m}($ age $)$ for the CARDIA study

Figure 5.2 displays the graphs of $\hat{m}(a g e)$ vs. age under three methods of estimation. The GEE provides a straight line to estimate the curve $\hat{m}($.$) , which makes sense$ because it used the identity function to estimate the nonlinear part of the model. While the other two methods provide slightly different graphs, the unweighted partially linear GEE (RSGEE ) provides two broken lines to estimate the nonlinear curve $m($.$) , and it does not provide a smooth curve, but our proposed weighted par-$ tially linear GEE (WRSGEE) method provides a much smoother curve to estimate the nonlinear function. 

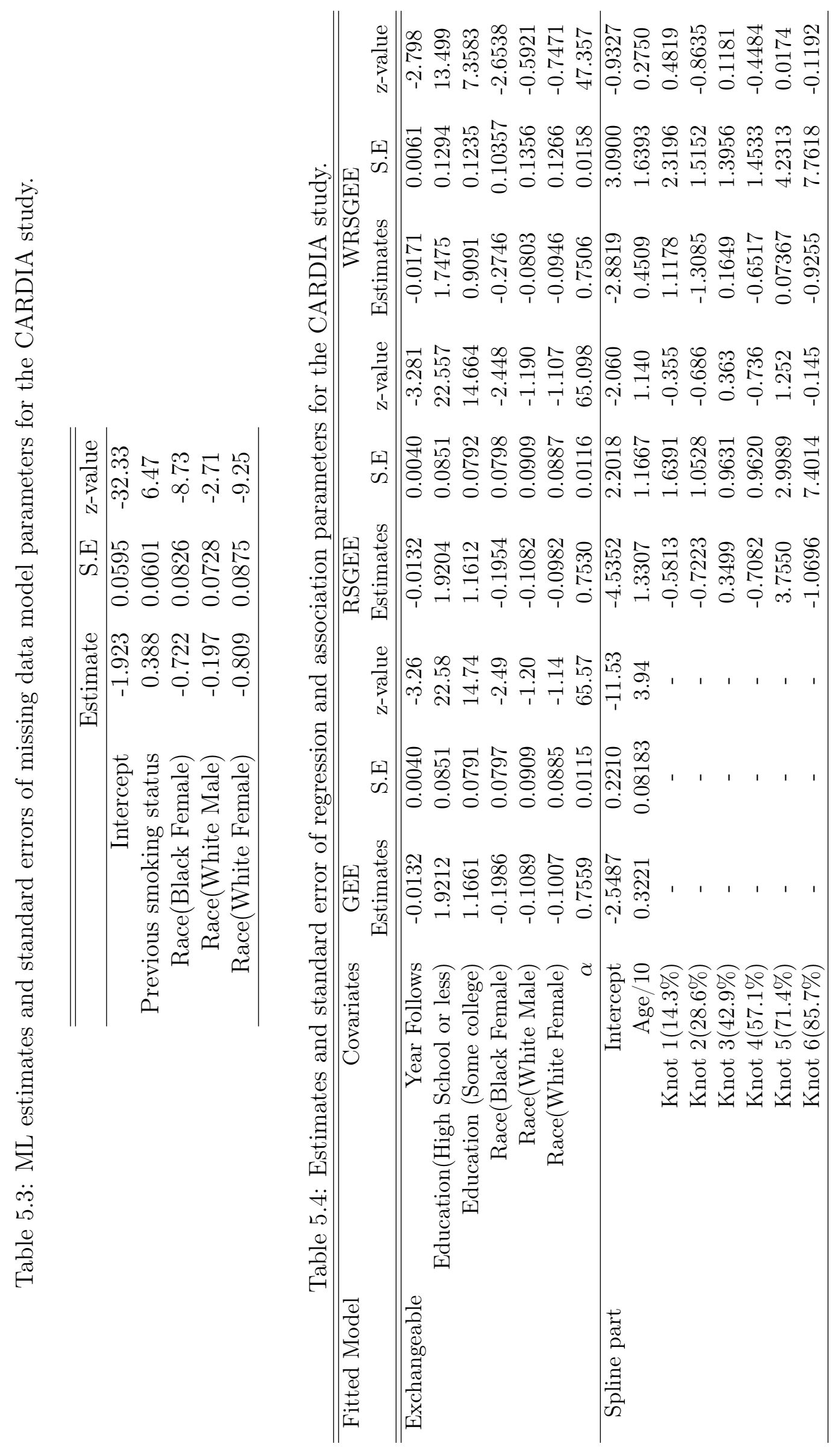
Table 5.5 contains the estimates of $m$ (Age) for different values of age. All the three methods provide slightly different results. For example, at Age $=26$, the GEE estimate for the nonlinear function is -1.71, while the RSGEE and WRSGEE estimate are -1.68 and -1.47 , respectively.

Table 5.5: The estimated nonlinear function $m(z)$ at different of $z(a g e / 10)$ for CARDIA study.

\begin{tabular}{lcrcc}
\hline \hline & & GEE & RSGEE & WRSGEE \\
& Age & $\hat{m}$ (Age) & $\hat{m}$ (Age) & $\hat{m}$ (Age) \\
\hline \hline Nonlinear function: & 1.8 & -1.97 & -2.14 & -2.07 \\
& 2.2 & -1.84 & -1.75 & -1.62 \\
& 2.5 & -1.74 & -1.72 & -1.52 \\
& 2.6 & -1.71 & -1.68 & -1.47 \\
& 2.7 & -1.68 & -1.65 & -1.43 \\
& 2.8 & -1.61 & -1.69 & -1.46 \\
& 3.0 & -1.58 & -1.67 & -1.48 \\
\hline
\end{tabular}




\subsection{Partially Linear Model for Incomplete Count Longitudinal Data}

\subsubsection{Partially Log-Linear Response Model}

Let $\mu_{i t}$ be the marginal mean response for the $i^{t h}$ individual at time $t$, that is,

$$
E\left(y_{i t} \mid \mathbf{x}_{i t}, z_{i t}\right)=\mu_{i t}, \quad i=1, \ldots, k, \quad t=1, \ldots, T .
$$

The marginal mean is assumed to have a log-linear relationship with the covariates in the form

$$
\log \left(\mu_{i t}\right)=\mathbf{x}_{i t}^{\prime} \boldsymbol{\beta}+m\left(z_{i t}\right)
$$

where $\boldsymbol{\beta}$ is a $p \times 1$ vector and $m($.$) is an unidentified smooth function. The marginal$ variance of $y_{i t}$ is given by

$$
\operatorname{var}\left(y_{i t} \mid \boldsymbol{x}_{i t}, z_{i t}\right)=\phi \mu_{i t}
$$

Assume that $y_{i t}$ and $y_{i^{\prime} t^{\prime}}$ are uncorrelated when $i \neq i^{\prime}$. Suppose

$$
\operatorname{corr}\left(y_{i t}, y_{i t^{\prime}}\right)=\alpha_{t t^{\prime}}
$$

denotes the correlation between $y_{i t}$ and $y_{i t^{\prime}}$ for given $\mathbf{x}_{i t}$, where $\boldsymbol{\alpha}=\left(\alpha_{12}, \ldots, \alpha_{1 T}\right.$, $\left.\alpha_{23}, \ldots, \alpha_{T-1, T}\right)^{\prime}$ is a vector of correlation parameters. If $m($.$) is specified as the$ identity function, then model (5.41) becomes an ordinary log-linear regression model. Note that the dropout model discussed in Section 5.2.2 is implemented here.

\subsubsection{Methods of Estimation}

\subsubsection{Weighted Regression Spline Generalized Estimating Equations (WRSGEEs)}

The estimating functions $\mathbf{U}_{\boldsymbol{\beta}^{*}}\left(\boldsymbol{\beta}^{*}, \boldsymbol{\vartheta}, m().\right)$ and $\mathbf{U}_{\boldsymbol{\vartheta}}\left(\boldsymbol{\beta}^{*}, \boldsymbol{\vartheta}, m().\right)$ for a partially linear model as defined in Section 4.5.2 contain an unidentified smooth function 
$m($.$) , which is approximated by a regression spline as discussed earlier.$

To deal with missing data, recall that we partitioned the count response $\mathbf{y}_{i}$ into observed and unobserved (missing) components as $\left(\mathbf{y}_{i}^{o}, \mathbf{y}_{i}^{m}\right)$. Similarly, we consider partitioning $\boldsymbol{\mu}_{i}$ as $\boldsymbol{\mu}_{i}=\left(\boldsymbol{\mu}_{i}^{o}, \boldsymbol{\mu}_{i}^{m}\right)$. Using the inverse probability weight of Robins et al. (1995), we propose a weighted second-order GEE approach and regression spline smoother for the simultaneous estimation of $m(),. \boldsymbol{\beta}^{*}$ and $\boldsymbol{\vartheta}$ for longitudinal count data with dropouts. The estimators of $m(),. \boldsymbol{\beta}^{*}$ and $\boldsymbol{\vartheta}$ are obtained by solving the equations

$$
\begin{gathered}
\hat{\mathbf{U}}_{\boldsymbol{\beta}^{*}}\left(\boldsymbol{\beta}^{*}, \boldsymbol{\vartheta}, m(.)\right)=\sum_{i=1}^{k} \frac{1}{\nu_{i m}} \mathbf{D}_{i}^{\prime} \mathbf{V}_{i}^{-1}\left(\mathbf{y}_{i}^{o}-\boldsymbol{\mu}_{i}^{o}\left(\boldsymbol{\beta}^{*}, \boldsymbol{\vartheta}\right)\right)=0 \\
\hat{\mathbf{U}}_{\boldsymbol{\vartheta}}\left(\boldsymbol{\beta}^{*}, \boldsymbol{\vartheta}, m(.)\right)=\sum_{i=1}^{k} \frac{1}{\nu_{i m}} \mathbf{L}_{i}^{\prime} \mathbf{W}_{i}^{-1}\left(\mathbf{r}_{i}^{o}-\boldsymbol{\delta}_{i}^{o}\right)=0
\end{gathered}
$$

where $\boldsymbol{\mu}_{i}^{o}\left(\boldsymbol{\beta}^{*}, \boldsymbol{\vartheta}\right)=\left(\mu_{i 1}^{o}\left(\boldsymbol{\beta}^{*}, \boldsymbol{\vartheta}\right), \ldots, \mu_{i T}^{o}\left(\boldsymbol{\beta}^{*}, \boldsymbol{\vartheta}\right)\right)^{\prime}$ with

$$
\mu_{i t}^{o}\left(\boldsymbol{\beta}^{*}, \boldsymbol{\vartheta}\right)=\exp \left(\mathbf{x}_{i t}^{\prime} \boldsymbol{\beta}+\hat{m}\left(z_{i t}\right)\right)
$$

\subsubsection{Estimating Dropout Probabilities}

Prior to solving the estimating equations (5.45) and (5.46), we estimate the response probability weights $\nu_{i m}$. The pseudo-likelihood function for $\boldsymbol{\tau}$ defined by

$$
\begin{gathered}
L(\boldsymbol{\tau})=\prod_{i=1}^{k} P\left(M_{i}=m_{i} \mid \mathbf{y}_{i t}^{*}, \boldsymbol{\tau}\right), \\
=\prod_{i=1}^{k}\left\{\prod_{t=2}^{m_{i}-1} \frac{1}{1+\exp \left(\mathbf{y}_{i t}^{*^{\prime}} \boldsymbol{\tau}\right)}\right\}\left\{\frac{\exp \left(\mathbf{y}_{i m_{i}}^{*^{\prime}} \boldsymbol{\tau}\right)}{1+\exp \left(\mathbf{y}_{i m_{i}}^{*^{\prime}} \boldsymbol{\tau}\right)}\right\}^{I\left\{m_{i} \leq T\right\}},
\end{gathered}
$$

where $\mathbf{y}_{i t}^{*}=\left(1, y_{i, t-1}, y_{i t}, \mathbf{x}_{i t}^{\prime}, z_{i t}\right)^{\prime}$ and $\boldsymbol{\tau}=\left(\tau_{0}, \tau_{1}, \tau_{2}, \ldots\right)^{\prime}$.

Let

$$
\operatorname{logit}\left\{P_{i t}^{*}(\boldsymbol{\tau})\right\}=\mathbf{y}_{i t}^{*^{\prime}} \boldsymbol{\tau}
$$

From (5.49), the pseudo-score equation for $\boldsymbol{\tau}$ takes the form 


$$
S(\boldsymbol{\tau})=\sum_{i=1}^{k}\left\{-\sum_{t=2}^{m_{i}-1} P_{i t}^{*}(\boldsymbol{\tau}) \mathbf{y}_{i t}^{*}+I\left\{m_{i} \leq T\right\}\left\{1-P_{i m_{i}}^{*}(\boldsymbol{\tau})\right\} \mathbf{y}_{i m_{i}}^{*}\right\}=0
$$

The Information matrix is obtained as

$$
I(\boldsymbol{\tau})=\sum_{i=1}^{k} \sum_{t=2}^{\min \left(m_{i}, T\right)} P_{i t}^{*}(\boldsymbol{\tau})\left(1-P_{i t}^{*}(\boldsymbol{\tau})\right) \mathbf{y}_{i t}^{*} \mathbf{y}_{i t^{\prime}}^{*}
$$

The Newton-Raphson iterative procedure is applied to solve the above estimating equations for the ML estimator $\hat{\boldsymbol{\tau}}=\left(\hat{\tau}_{0}, \hat{\tau}_{1}, \hat{\tau}_{2}, \ldots\right)^{\prime}$. For the $i^{\text {th }}$ individual at time $t$, the predicted probability of response is obtained as

$$
1-\hat{P}_{i t}^{*}=\frac{1}{1+\exp \left(\mathbf{y}_{i t}^{\prime *} \hat{\boldsymbol{\tau}}\right)}
$$

Then for the $i^{\text {th }}$ individual at time $m_{i}$, the dropout probability is estimated by

$$
\left.\hat{\nu}_{i m_{i}}=P\left(M_{i}=m_{i} \mid \hat{\mathbf{y}}_{i t}^{* \prime}, \hat{\boldsymbol{\tau}}\right)=\left\{\prod_{t=2}^{m_{i}-1}\left(1-\hat{P}_{i t}^{*}\right)\right\} \times\left\{\hat{P}_{i m_{i}}^{*}\right)\right\}^{I\left\{m_{i} \leq T\right\}} .
$$

Then in equations (5.45) and (5.46), we replace $\nu_{i m}$ 's by $\hat{\nu}_{i m}$ and iteratively solve these equations.

\subsubsection{Computational Details for Estimating the Response Model}

Recall the mean response model (5.42). We approximate the nonlinear function by the regression spline

$$
\hat{m}\left(z_{i t}\right)=\Phi_{i t}^{\prime} \gamma
$$

where

$$
\log \left(\mu_{i t}\right)=\mathbf{x}_{i t}^{*^{\prime}} \boldsymbol{\beta}^{*}=\mathbf{x}_{i t}^{\prime} \boldsymbol{\beta}+\Phi_{i t}^{\prime} \boldsymbol{\gamma}
$$

or

$$
\mu_{i t}=\exp \left(\mathbf{x}_{i t}^{*^{\prime}} \boldsymbol{\beta}^{*}\right)
$$


The iterative procedure for computing the estimates of the parameters $\left(\boldsymbol{\beta}^{*}, \boldsymbol{\vartheta}\right)$ initiates with some preliminary values $\left(\hat{\boldsymbol{\beta}}_{0}^{*}, \hat{\boldsymbol{\vartheta}}_{0}\right)$. We solve equations (5.45) and (5.46) by using the iterative procedure:

$$
\begin{aligned}
& \hat{\boldsymbol{\beta}}_{s+1}^{*}=\hat{\boldsymbol{\beta}}_{s}^{*}+\left(\sum_{i=1}^{k} \frac{1}{\nu_{i m}} \mathbf{D}_{i}^{\prime} \mathbf{V}_{i}^{-1} \mathbf{D}_{i}\right)^{-1} \sum_{i=1}^{k} \frac{1}{\nu_{i m}} \mathbf{D}_{i}^{\prime} \mathbf{V}_{i}^{-1}\left(\mathbf{y}_{i}^{o}-\boldsymbol{\mu}_{i}^{o}\right), \\
& \hat{\boldsymbol{\vartheta}}_{s+1}=\hat{\boldsymbol{\vartheta}}_{s}+\left(\sum_{i=1}^{k} \frac{1}{\nu_{i m}} \mathbf{L}_{i}^{\prime} \mathbf{W}_{i}^{-1} \mathbf{L}_{i}\right)^{-1} \sum_{i=1}^{k} \frac{1}{\nu_{i m}} \mathbf{L}_{i}^{\prime} \mathbf{W}_{i}^{-1}\left(\mathbf{r}_{i}^{o}-\boldsymbol{\delta}_{i}^{o}\right),
\end{aligned}
$$

for $s=0,1,2, \ldots$, where the second expression on the right side of every estimating equation is evaluated at the present estimates $\left(\hat{\boldsymbol{\beta}}_{s}^{*}, \hat{\boldsymbol{\vartheta}}_{s}\right)$.

\subsubsection{Simulation Study}

We ran a series of simulations using incomplete count longitudinal data. Following three methods were compared in the simulation study.

1. GEE: Ordinary GEE approach of Prentice (1988) is used to find estimates of $\boldsymbol{\beta}, \boldsymbol{\alpha}, \phi$, and $m($.$) , where m($.$) is considered as a linear function.$

2. RSGEE: The unweighted regression spline GEE method is used to estimate $\boldsymbol{\beta}, \boldsymbol{\alpha}, \phi$, and unknown smooth function $m($.$) .$

3. WRSGEE: The proposed weighted regression spline GEE method is used to estimate $\boldsymbol{\beta}, \boldsymbol{\alpha}, \phi$, and unknown smooth function $m($.$) . We incorporate the in-$ verse probability weights of Robins et al. (1995) into our semiparametric method of estimation.

\subsubsection{Correlated Count Data}

The generalized partial linear mixed model (GPLMM) as discussed in Chapter 4 is used to produce a large correlated count data set with some pre-specified function of the marginal mean. The true population parameters are obtained by fit- 
ting the marginal model with GEE. We generate replicates of data sets by drawing subsamples from the above data set for the number of subjects $k=300,500$ and 1000.

\subsubsection{Response Model}

We consider measures of count response on three occasions. The marginal mean response $E\left(y_{i t} \mid \mathbf{x}_{i t}, \boldsymbol{\beta}^{*}, \alpha, \phi\right)$ is given by

$$
\log \left[E\left(y_{i t} \mid \mathbf{x}_{i t}, \boldsymbol{\beta}^{*}, \alpha, \phi\right)\right]=\beta_{1} x_{i}+\beta_{2} t+m\left(z_{i t}\right) .
$$

The covariate vector $\mathbf{x}_{i t}=\left(x_{i}, t, z_{i t}\right)^{\prime}$, where $x_{i}$ is a treatment group indicator that takes the value 1 for half of the subjects and 0 for the other half. We produced $z_{i t}$ from the uniform distribution $U[0,1]$, and $t$ denotes the time points $1,2,3$ for each subject. The function $m()=.\beta_{1}+(0.2 * z /(0.05+z))$ was estimated based on the following spline function

$$
s(z, \gamma)=\gamma_{0}+\gamma_{1} z+\sum_{r=1}^{4} \gamma_{r+1}\left(z-\zeta_{r}\right)_{+},
$$

where $\gamma=\left(\gamma_{3}, \gamma_{4}, \gamma_{5}, \ldots, \gamma_{6}\right)^{\prime}$ are the spline basis coefficients.

Two different sets of simulations were run. In the first set, the true values of the parameters were considered to be $\left(\beta_{1}, \beta_{2}\right)=(0.5,0.3), \alpha=0.16$, and $\phi=1.2$, while for the second set of simulations, the true values were $\left(\beta_{1}, \beta_{2}\right)=(0.2,0.1), \alpha=$ 0.12 , and $\phi=1.14$. For both studies, exchangeable correlations were used, where each simulation run was based on 1000 replicates of data sets, with each data set containing $k=300,500$ and 1000 subjects. 


\subsubsection{Dropout Model}

The missing data indicator $R_{i t}=1$ if $y_{i t}$ is observed, and 0 , if $y_{i t}$ is missing. We assume

$$
P\left(R_{i t}=0 \mid R_{i t}=\cdots=R_{i, t-1}=1, y_{i 1}, \ldots, y_{i t-1}, \boldsymbol{\tau}\right)=\frac{\exp \left(\psi_{i t}\right)}{1+\exp \left(\psi_{i t}\right)},
$$

where $\psi_{i t}=\tau_{0}+\tau_{1} y_{i t-1}+\tau_{2} y_{i t}$, for $t=2,3$, The true parameter vector $\left(\tau_{0}, \tau_{1}, \tau_{2}\right)^{\prime}$

was taken as $(-3,0.6,0)^{\prime}$ and $(-3,0.8,0)^{\prime}$ for two scenarios, which yield about $30 \%$ missingness in our response model. For the above model, we have

$\nu_{i m_{i}}=P\left(M_{i}=m_{i} \mid y_{i 1}, \ldots, y_{i m_{i}}, \boldsymbol{\tau}\right)=\left\{\prod_{t=2}^{m_{i}-1} \frac{1}{1+\exp \left(\psi_{i t}\right)}\right\}\left\{\frac{\exp \left(\psi_{i m_{i}}\right)}{1+\exp \left(\psi_{i m_{i}}\right)}\right\}^{I\left\{m_{i} \leq T\right\}}$.

Note that the choice $\tau_{2}=0$ leads to a MAR Model.

\subsubsection{Results}

Tables 5.6 and 5.7 present empirical biases and mean squared errors (MSEs) of the GEE, RSGEE, and WRSGEE estimators of regression parameters $\left(\beta_{1}, \beta_{2}\right)$, correlation parameter $\alpha$, dispersion parameters $\phi$, and nonlinear function $m(z)$ at different values of $z=(0.01,0.02,0.25,0.55,0.99)$, and for $\alpha=0.16$ and $\alpha=0.12$. 
Table 5.6: Empirical biases and mean squared errors (MSEs) of GEE, RSGEE and WRSGEE estimators of regression and association parameters.

\begin{tabular}{|c|c|c|c|c|c|c|c|}
\hline \multirow[b]{3}{*}{ Parameter } & \multicolumn{7}{|c|}{ Partially Linear Model for $\boldsymbol{\tau}=(-3,0.6,0)$ and $\mathbf{k}=\mathbf{3 0 0}$} \\
\hline & \multirow[b]{2}{*}{ True value } & \multicolumn{2}{|c|}{ GEE } & \multicolumn{2}{|c|}{ RSGEE } & \multicolumn{2}{|c|}{ WRSGEE } \\
\hline & & Bias & MSE & Bias & MSE & Bias & MSE \\
\hline$\alpha$ & 0.16 & -0.0204 & 0.0037 & -0.0200 & 0.0037 & -0.0143 & 0.0104 \\
\hline$\phi$ & 1.2 & -0.1410 & 0.0238 & -0.1483 & 0.0258 & -0.0974 & 0.0239 \\
\hline$\beta_{1}$ & 0.5 & -0.0068 & 0.0021 & -0.0067 & 0.0021 & -0.0135 & 0.0060 \\
\hline$\beta_{2}$ & 0.2 & -0.0043 & 0.0008 & -0.0046 & 0.0008 & 0.0017 & 0.0019 \\
\hline$m\left(z_{1}\right)$ & 0.53 & 0.1310 & 0.0223 & 0.0691 & 0.0166 & 0.0531 & 0.0287 \\
\hline$m\left(z_{2}\right)$ & 0.55 & 0.1080 & 0.0168 & 0.0505 & 0.0133 & 0.0348 & 0.0248 \\
\hline$m\left(z_{3}\right)$ & 0.66 & 0.0165 & 0.0044 & 0.0371 & 0.0069 & 0.0267 & 0.0157 \\
\hline$m\left(z_{4}\right)$ & 0.68 & 0.0233 & 0.0043 & 0.0330 & 0.0068 & 0.0191 & 0.0150 \\
\hline \multirow[t]{2}{*}{$m\left(z_{5}\right)$} & 0.69 & 0.0506 & 0.0077 & 0.0308 & 0.0115 & 0.0144 & 0.0269 \\
\hline & \multicolumn{7}{|c|}{ Partially Linear Model for $\boldsymbol{\tau}=(-3,0.6,0)$ and $\mathbf{k}=\mathbf{5 0 0}$} \\
\hline \multirow[b]{2}{*}{ Parameter } & \multirow[b]{2}{*}{ True value } & \multicolumn{2}{|c|}{ GEE } & \multicolumn{2}{|c|}{ RSGEE } & \multicolumn{2}{|c|}{ WRSGEE } \\
\hline & & Bias & MSE & Bias & MSE & Bias & MSE \\
\hline$\alpha$ & 0.16 & -0.0187 & 0.0022 & -0.0182 & 0.0022 & -0.0080 & 0.0076 \\
\hline$\phi$ & 1.2 & -0.1398 & 0.0221 & -0.1446 & 0.0234 & -0.0701 & 0.0172 \\
\hline$\beta_{1}$ & 0.5 & -0.0040 & 0.0013 & -0.0039 & 0.0013 & -0.0084 & 0.0041 \\
\hline$\beta_{2}$ & 0.2 & -0.0044 & 0.0005 & -0.0044 & 0.0005 & 0.0033 & 0.0013 \\
\hline$m\left(z_{1}\right)$ & 0.53 & 0.1314 & 0.0204 & 0.0672 & 0.0111 & 0.0516 & 0.0229 \\
\hline$m\left(z_{2}\right)$ & 0.55 & 0.1084 & 0.0149 & 0.0488 & 0.0084 & 0.0333 & 0.0194 \\
\hline$m\left(z_{3}\right)$ & 0.66 & 0.0165 & 0.0028 & 0.0390 & 0.0049 & 0.0242 & 0.0105 \\
\hline$m\left(z_{4}\right)$ & 0.68 & 0.0228 & 0.0029 & 0.0303 & 0.0044 & 0.0146 & 0.0101 \\
\hline \multirow[t]{3}{*}{$m\left(z_{5}\right)$} & 0.69 & 0.0495 & 0.0056 & 0.0298 & 0.0077 & 0.0075 & 0.0188 \\
\hline & \multicolumn{7}{|c|}{ Partially Linear Model for $\boldsymbol{\tau}=(-3,0.6,0)$ and $\mathbf{k}=\mathbf{1 0 0 0}$} \\
\hline & \multicolumn{3}{|c|}{ GEE } & \multicolumn{2}{|c|}{ RSGEE } & \multicolumn{2}{|c|}{ WRSGEE } \\
\hline Parameter & True value & Bias & MSE & Bias & MSE & Bias & MSE \\
\hline$\alpha$ & 0.16 & -0.0208 & 0.0013 & -0.0205 & 0.0013 & -0.0066 & 0.0053 \\
\hline$\phi$ & 1.2 & -0.1397 & 0.0207 & -0.1426 & 0.0216 & -0.0515 & 0.0114 \\
\hline$\beta_{1}$ & 0.5 & -0.0039 & 0.0006 & -0.0039 & 0.0006 & -0.0038 & 0.0023 \\
\hline$\beta_{2}$ & 0.2 & -0.0056 & 0.0003 & -0.0057 & 0.0003 & 0.0013 & 0.0007 \\
\hline$m\left(z_{1}\right)$ & 0.53 & 0.1325 & 0.0191 & 0.0713 & 0.0086 & 0.0568 & 0.0136 \\
\hline$m\left(z_{2}\right)$ & 0.55 & 0.1095 & 0.0135 & 0.0528 & 0.0060 & 0.0383 & 0.0109 \\
\hline$m\left(z_{3}\right)$ & 0.66 & 0.0177 & 0.0015 & 0.0397 & 0.0032 & 0.0257 & 0.0061 \\
\hline$m\left(z_{4}\right)$ & 0.68 & 0.0241 & 0.0017 & 0.0320 & 0.0026 & 0.0179 & 0.0058 \\
\hline$m\left(z_{5}\right)$ & 0.69 & 0.0510 & 0.0041 & 0.0328 & 0.0044 & 0.0173 & 0.0115 \\
\hline
\end{tabular}


Table 5.7: Empirical biases and mean squared errors (MSEs) of GEE, RSGEE and WRSGEE estimators of regression and association parameters.

\begin{tabular}{|c|c|c|c|c|c|c|c|}
\hline \multirow[b]{3}{*}{ Parameter } & \multicolumn{5}{|c|}{ Partially Linear Model for $\boldsymbol{\tau}=(-3,0.8,0)$ and $\mathbf{k}=\mathbf{3 0 0}$} & \multirow{2}{*}{\multicolumn{2}{|c|}{ WRSGEE }} \\
\hline & \multirow[b]{2}{*}{ True value } & \multicolumn{2}{|c|}{ GEE } & \multicolumn{2}{|c|}{ RSGEE } & & \\
\hline & & Bias & MSE & Bias & MSE & Bias & MSE \\
\hline$\alpha$ & 0.12 & -0.0219 & 0.0032 & -0.0216 & 0.0033 & -0.0171 & 0.0092 \\
\hline$\phi$ & 1.14 & -0.1526 & 0.0266 & -0.1582 & 0.0284 & -0.1065 & 0.0223 \\
\hline$\beta_{1}$ & 0.2 & -0.0009 & 0.0028 & -0.0010 & 0.0028 & -0.0085 & 0.0092 \\
\hline$\beta_{2}$ & 0.1 & -0.0045 & 0.0011 & -0.0047 & 0.0011 & 0.0054 & 0.0030 \\
\hline$m\left(z_{1}\right)$ & 0.53 & 0.1277 & 0.0228 & 0.0622 & 0.0191 & 0.0406 & 0.0476 \\
\hline$m\left(z_{2}\right)$ & 0.55 & 0.1046 & 0.0174 & 0.0438 & 0.0158 & 0.0222 & 0.0424 \\
\hline$m\left(z_{3}\right)$ & 0.66 & 0.0124 & 0.0052 & 0.0337 & 0.0081 & 0.0119 & 0.0226 \\
\hline$m\left(z_{4}\right)$ & 0.68 & 0.0182 & 0.0048 & 0.0274 & 0.0072 & 0.0081 & 0.0241 \\
\hline$m\left(z_{5}\right)$ & 0.69 & 0.0442 & 0.0081 & 0.0259 & 0.0142 & 0.0003 & 0.0390 \\
\hline
\end{tabular}

\begin{tabular}{|c|c|c|c|c|c|c|c|}
\hline \multirow[b]{3}{*}{ Parameter } & \multicolumn{7}{|c|}{ Partially Linear Model for $\boldsymbol{\tau}=(-3,0.8,0)$ and $\mathbf{k}=\mathbf{5 0 0}$} \\
\hline & \multirow[b]{2}{*}{ True value } & \multicolumn{2}{|c|}{ GEE } & \multicolumn{2}{|c|}{ RSGEE } & \multicolumn{2}{|c|}{ WRSGEE } \\
\hline & & Bias & MSE & Bias & MSE & Bias & MSE \\
\hline$\alpha$ & 0.12 & -0.0212 & 0.0023 & -0.0210 & 0.0024 & -0.0103 & 0.0083 \\
\hline$\phi$ & 1.14 & -0.1504 & 0.0246 & -0.1543 & 0.0257 & -0.0790 & 0.0153 \\
\hline$\beta_{1}$ & 0.2 & 0.0004 & 0.0016 & 0.0005 & 0.0016 & -0.0042 & 0.0064 \\
\hline$\beta_{2}$ & 0.1 & -0.0062 & 0.0006 & -0.0064 & 0.0007 & 0.0005 & 0.0020 \\
\hline$m\left(z_{1}\right)$ & 0.53 & 0.1283 & 0.0201 & 0.0620 & 0.0120 & 0.0428 & 0.0294 \\
\hline$m\left(z_{2}\right)$ & 0.55 & 0.1053 & 0.0147 & 0.0438 & 0.0093 & 0.0252 & 0.0258 \\
\hline$m\left(z_{3}\right)$ & 0.66 & 0.0140 & 0.0031 & 0.0364 & 0.0054 & 0.0250 & 0.0139 \\
\hline$m\left(z_{4}\right)$ & 0.68 & 0.0211 & 0.0031 & 0.0320 & 0.0051 & 0.0168 & 0.0149 \\
\hline$m\left(z_{5}\right)$ & 0.69 & 0.0489 & 0.0062 & 0.0315 & 0.0085 & 0.0177 & 0.0275 \\
\hline
\end{tabular}

\begin{tabular}{|c|c|c|c|c|c|c|c|}
\hline \multirow[b]{3}{*}{ Parameter } & \multicolumn{7}{|c|}{ Partially Linear Model for $\boldsymbol{\tau}=(-3,0.8,0)$ and $\mathbf{k}=\mathbf{1 0 0 0}$} \\
\hline & \multirow[b]{2}{*}{ True value } & \multicolumn{2}{|c|}{ GEE } & \multicolumn{2}{|c|}{ RSGEE } & \multicolumn{2}{|c|}{ WRSGEE } \\
\hline & & Bias & MSE & Bias & MSE & Bias & MSE \\
\hline$\alpha$ & 0.12 & -0.0230 & 0.0014 & -0.0228 & 0.0014 & -0.0105 & 0.0053 \\
\hline$\phi$ & 1.14 & -0.1462 & 0.0225 & -0.1486 & 0.0232 & -0.0486 & 0.0109 \\
\hline$\beta_{1}$ & 0.2 & -0.0024 & 0.0008 & -0.0024 & 0.0008 & -0.0050 & 0.0038 \\
\hline$\beta_{2}$ & 0.1 & -0.0066 & 0.0004 & -0.0067 & 0.0004 & -0.0001 & 0.0012 \\
\hline$m\left(z_{1}\right)$ & 0.53 & 0.1313 & 0.0192 & 0.0685 & 0.0094 & 0.0558 & 0.0208 \\
\hline$m\left(z_{2}\right)$ & 0.55 & 0.1082 & 0.0136 & 0.0500 & 0.0068 & 0.0374 & 0.0175 \\
\hline$m\left(z_{3}\right)$ & 0.66 & 0.0170 & 0.0018 & 0.0385 & 0.0036 & 0.0282 & 0.0099 \\
\hline$m\left(z_{4}\right)$ & 0.68 & 0.0241 & 0.0020 & 0.0339 & 0.0033 & 0.0228 & 0.0097 \\
\hline$m\left(z_{5}\right)$ & 0.69 & 0.0520 & 0.0047 & 0.0338 & 0.0056 & 0.0217 & 0.0174 \\
\hline
\end{tabular}


It is evident from the results that the proposed WRSGEE method generally provides unbiased estimates of the model parameters under all scenarios considered in the simulation study. The GEE method provides systematic biases, specifically for the estimates of the dispersion parameters $\phi$ and nonlinear function $m($.$) . For$ example, Table 5.6 shows that when estimating $\phi$ at $k=500$, the GEE method provides percentage relative bias of $(-0.1398 / 1.2) 100=-11.65 \%$, where the WRSGEE method provides a smaller percentage relative bias of $(-0.0701 / 1.2) 100=5.84 \%$.

Also, Table 5.7 shows that when estimating $m\left(z_{2}\right)$ at $k=500$, the GEE method provides a percentage relative bias of $(0.1053 / 0.55) 100=19.15 \%$, where the RSGEE and WRSGEE methods provide percentage relative bias of $(0.0438 / 0.55) 100=$ $7.96 \%$, and $(0.0252 / 0.55) 100=4.58 \%$, respectively. The unweighted RSGEE method appears to perform slightly better than the ordinary GEE method, but the proposed WRSGEE method clearly outperforms the other two methods considered. 


\section{Chapter 6}

\section{Application: Health and Retirement Study (HRS)}

\subsection{Introduction}

An analysis of Health and Retirement data is presented in this chapter. The institute for social research (ISR) conducted a longitudinal domiciliary survey, referred to as the Health and Retirement Study (HRS) (HRS, 2019), at the University of Michigan, USA. This multifaceted data set provides an opportunity for researchers to explore several features associated with the elderly population. The HRS is a widespread group individual survey with their spouses of age over 50 years. Its chief objective is to provide group data (panel data) that facilitate analysis and research in the provision of strategies on retirement, assets, health protection and financial security. The information extracted through this survey is about assets, demographics, health, housing, cognition, the structure of families and their bonding, the status of jobs and their history, insurance, utilization of health care and expenses, expectations and income.

The RAND HRS (2019) longitudinal file is an easily manageable subsection of the HRS. It includes cleaned and refined variables with conventions of naming compatible and intuitive. The National Institute of Aging (NIA) primarily sponsored 
HRS, with other additional aid by the Institute for Social Research (ISR). The study comprises of seven cohorts: the preliminary cohort of HRS initiated in 1931 and lasted to 1941. The first interview of this group was held in 1992 and then they were interviewed every two years afterwards.

\subsubsection{RAND HRS DATA Files Structure:}

The RAND HRS (2019) longitudinal data file consists of all seven cohorts. This documentation alludes to the whole survey, e.g. HRS and the cohort of 1931 - 41, which was interviewed first in 1992 and was characterized as the "original" or "initial" cohort of HRS entry. The RAND HRS (2019) longitudinal data file only includes the fundamental interviews and excluded departure interviews or any restricted data. The data were collected from 13 waves of interviews across 15 survey years (1992, 1993, 1994, 1995, and biennially 1996 - 2016). All the products of RAND data are accessible through the website of HRS (http://hrsonline.isr.umich.edu/data/ index.html).

A subsection of longitudinal data from RAND HRS (2019) is considered in this study, which includes the most recent 4 waves 10, 11, 12, 13 for the years 2010, 2012, 2014 and 2016. We consider wave 10 as our baseline wave. The number of visits with a medical doctor in the last two years by a respondent is considered as our response variable (RwDOCTIM). This study aims to recognize subgroups of respondents with similar behaviours in terms of the number of visits to a doctor's office, and to clarify what predictors affect the number of visits and how.

Our initial analysis of the data detected some influential observations and outliers in the response variables, which had a great impact on the analysis. To get a clear result of the analysis, we only considered respondents with maximum doctor visits of 40 or less. We further find that the data set comprises dropouts in the response variable and covariates. We assume that this drop-out process follows a MAR mechanism.

In this study, we expanded our proposed semiparametric approach to analyze 
incomplete longitudinal data with dropouts. The initial data analysis exhibits a nonlinear relationship between the covariate age and response. To analyze incomplete data, we used our weighted semiparametric partially linear method as proposed in Chapter 5. Here we jointly estimate the marginal mean, association and dispersion parameters by the weighted regression spline generalized estimating equations (WRSGEE).

\subsubsection{Baseline Covariates}

The survey data set consists of a total of 8670 respondents along with monotone missing observations and doctor visits of at most 40. We consider the following baseline covariates in our response model:

Age: age (in years) of respondent

Smoke: indicates whether the respondent ever smoked cigarettes or not

HBP: indicator of high blood pressure

Cancer: indicator of cancer

Heart: indicator of heart disease

Lung: indicator of lung condition

Gender: 1 = male, 0 = female

Hosp: indicator of overnight hospital stay in reference period

Depress: indicator of depression over a two-week period before the interview

BMI: body mass index

White: 1 , if a patient is white/Caucasian and 0 , otherwise

Black: 1, if a patient is black/African American and 0, otherwise

All records in which one of the above predictors do not contain an actual value have been removed. Specifically, we removed undefined categories, such as "Not Applicable", "Refusal", "Not Stated", "Don’t know", "Reference person did not respond to this wave", and "Other missing". A total of 12 records were removed from the predictor Hosp for not having an actual value. The predictor Lung did 
not have an actual value in 58 records. Also, 77 records are removed from the covariate Heart, while a total of 26 records were removed from the covariate Cancer. Additionally, a total of 3248 records were removed from BMI, 66 from HBP, 64 from Smoke and 298 cases from Depress, and 1 from Race. The total number of subjects at baseline is 4814 . Roughly $20 \%$ respondents have missing observations due to drop-out.

Figure 6.1 (a) shows the scatter plot of mean number of visits vs. age. It indicates a curvilinear relationship between the response and covariate age. The plot indicates some outliers between age $30-35$.

Figure 6.1 (b) shows the box-plots of the number of doctor visits in four waves. It is evident that the median numbers of doctor visits are similar across the four waves.

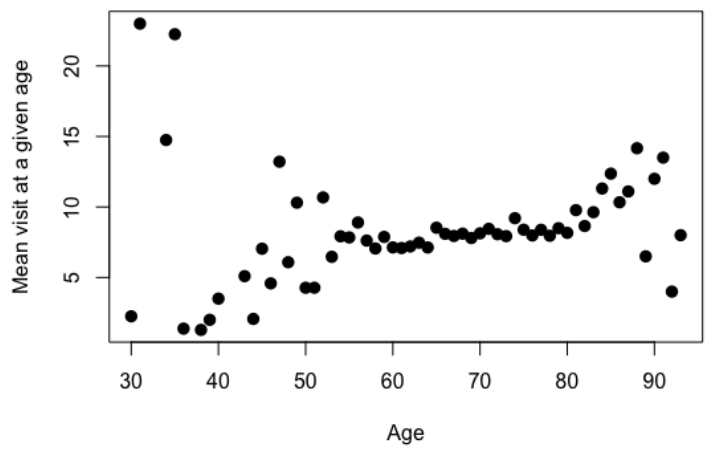

(a) Mean visit vs. Age

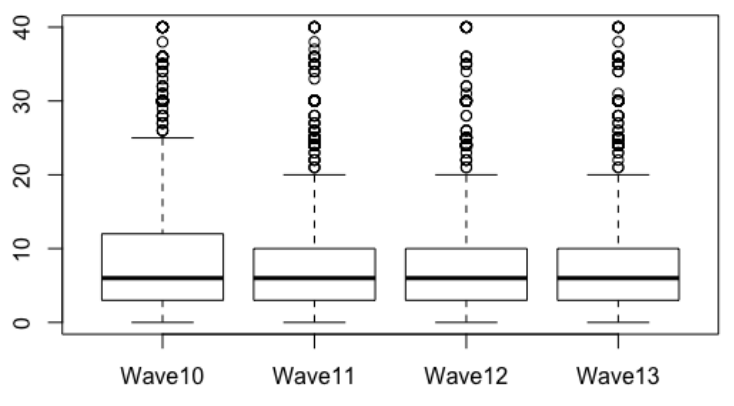

(b) Box plot

Figure 6.1: (a) Scatter plot of mean visits vs. age. (b) Box plots of doctor visits. 
Table 6.1 displays the summary statistics of the data set.

Table 6.1: Summary Statistics of RAND HRS Data

\begin{tabular}{|c|c|c|}
\hline $\begin{array}{l}\text { Subset file: RAND HRS Data } \\
\text { Total subjects }=4814 \\
\text { Total waves(Time) }=4\end{array}$ & $\begin{array}{l}\text { Doctor visits } \\
\text { Mean }=8 \\
\text { Var }=54.11 \\
\min =0 \\
\max =40 \\
\mathrm{NA}=3857(20 \%)\end{array}$ & $\begin{array}{l}\text { Age } \\
\text { Mean }=69.6 \\
\operatorname{Var}=49.4 \\
\min =30 \\
\max =93\end{array}$ \\
\hline $\begin{array}{l}\text { Lung } \\
\text { Mean }=0.114 \\
\text { Yes }(1)=547 \\
\text { No }(0)=4267\end{array}$ & $\begin{array}{l}\text { BMI } \\
\text { Mean }=28.5 \\
\operatorname{Var}=34.8 \\
\min =11.8 \\
\operatorname{Max}=74.7\end{array}$ & $\begin{array}{l}\text { Smoke } \\
\text { Mean }=0.584 \\
\text { Yes }(1)=2813 \\
\text { No }(0)=2001\end{array}$ \\
\hline $\begin{array}{l}\text { Depress } \\
\text { Mean }=0.107 \\
\text { Yes }(1)=514 \\
\text { No(0) }=4300\end{array}$ & $\begin{array}{l}\text { Cancer } \\
\text { Mean }=0.166 \\
\text { Yes }(1)=797 \\
\mathrm{No}(0)=4017\end{array}$ & $\begin{array}{l}\text { Heart } \\
\text { Mean }=0.258 \\
\text { Yes }(1)=1243 \\
\mathrm{No}(0)=3571\end{array}$ \\
\hline $\begin{array}{l}\text { HBP } \\
\text { Mean }=0.660 \\
\text { Yes }(1)=3179 \\
\text { No }(0)=1635\end{array}$ & $\begin{array}{l}\text { Gender } \\
\text { Mean }=0.43 \\
\text { Meal }(1)=2072 \\
\text { Female }(0)=2742\end{array}$ & $\begin{array}{l}\text { White Caucasian } \\
\text { Mean }=0.812 \\
\text { Yes }(1)=3907 \\
\text { No }(0)=907\end{array}$ \\
\hline $\begin{array}{l}\text { Black African American } \\
\text { Mean }=0.14 \\
\text { Yes }(1)=684 \\
\text { No }(0)=4130\end{array}$ & & \\
\hline
\end{tabular}




\subsubsection{Response Model}

Let $y_{i t}$ be the number of visits to a doctor's office by the $i^{\text {th }}$ respondent/patient at the $t^{\text {th }}$ time, $i=1, \ldots, 4814$ and $t=1, \ldots, 4$. The marginal mean response $\mu_{i t}=E\left(y_{i t}\right)$ is defined as a function of covariates by

$$
\begin{aligned}
\log \left(\mu_{i t}\right)=\beta_{0}+ & \beta_{1} \text { Smoke }_{i}+\beta_{2} \text { Lung }_{i}+\beta_{3} \text { Hosp }_{i}+\beta_{4} \text { Cancer }_{i} \\
& +\beta_{5} \text { Depress }_{i}+\beta_{6} \text { Gender }_{i}+\beta_{7} \text { Heart }_{i}+\beta_{8} \text { White }_{i} \\
& +\beta_{9} \text { Black }_{i}+\beta_{10} \text { BMI }_{i}+\beta_{11} t+m\left(\left({\text { Age } \left./ 10)_{i}\right)}\right.\right.
\end{aligned}
$$

where $m($.$) is an unknown function. For analysis, the variable age is allowed to$ enter the model as a regression spline with 5 knots. The estimating equations were developed assuming an exchangeable association structure. Our proposed method used quadratic spline approximation $(l=2)$ for $m($ age $)$ with 5 knots.

\subsubsection{Missing Data Model}

To estimate the model parameters in equation (6.1) by the WRSGEE method, we first estimate the inverse probability weights based on the missing data model

$$
\begin{aligned}
\operatorname{logit}\left(p_{i t}^{*}\right)=\tau_{0}+\tau_{1} y_{i, t-1} & +\tau_{2}\left(\text { Age }_{10}\right)_{i}+\tau_{3} \text { Cancer }_{i}+\tau_{4} \text { Heart }_{i} \\
& +\tau_{5} \text { Lung }_{i}+\tau_{6} \text { Smoke }_{i}+\tau_{7} \text { White }_{i}+\tau_{8} \text { black }_{i}+\tau_{9} t
\end{aligned}
$$

where $p_{i t}^{*}=P\left(R_{i t}=0 \mid R_{i 1}=, \ldots, R_{i, t-1}=1, \mathbf{y}_{i}, \mathbf{x}_{i}, \boldsymbol{\tau}\right)$ is dropout time for the $i^{t h}$ individual at time $t$.

\subsubsection{Estimation Methods}

The following three methods were compared in the HRS data analysis:

1. GEE: Estimators of regression, association and dispersion parameters $\boldsymbol{\beta}, \boldsymbol{\alpha}$, and $\phi$, are obtained by solving the unweighted of Prentice (1988), where the function 
$m($ age $)$ is treated as a linear function of age.

2. RSGEE: The unweighted regression spline GEE method is used to estimate $\boldsymbol{\beta}, \boldsymbol{\alpha}, \phi$, and unknown smooth function $m($.$) .$

3. WRSGEE: The proposed weighted regression spline GEE method is used to estimate $\boldsymbol{\beta}, \boldsymbol{\alpha}, \phi$, and $m($.$) .$

Table (6.2) presents pseudo-ML estimates, their standard errors, and z-values of missing data model parameters for the HRS data. Table 6.3 presents the GEE, RSGEE and WRSGEE estimates of the regression, association and dispersion parameters, their standard errors and the corresponding z-values, while Table 6.4 shows the estimated values of spline function for different ages.

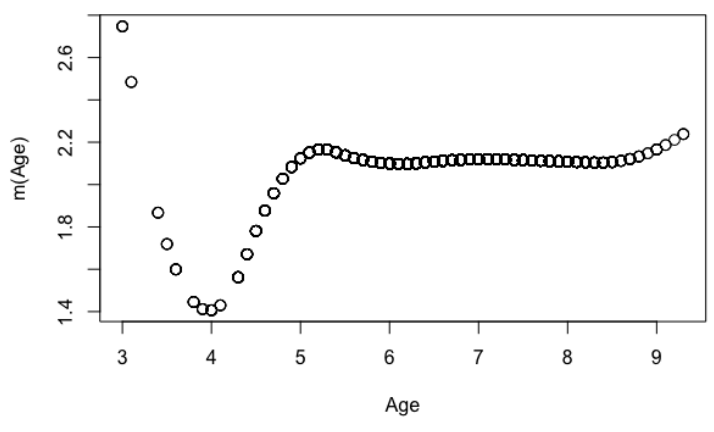

(a) WRSGEE

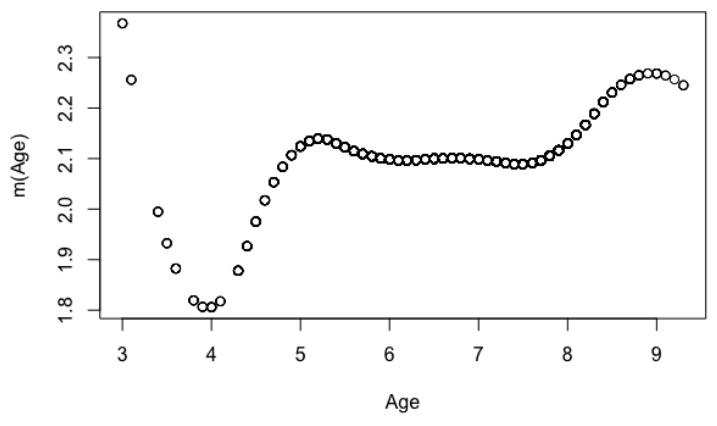

(b) RSGEE

Figure 6.2: Estimated nonlinear curve for HRS data analysis 


\section{Residual plots}

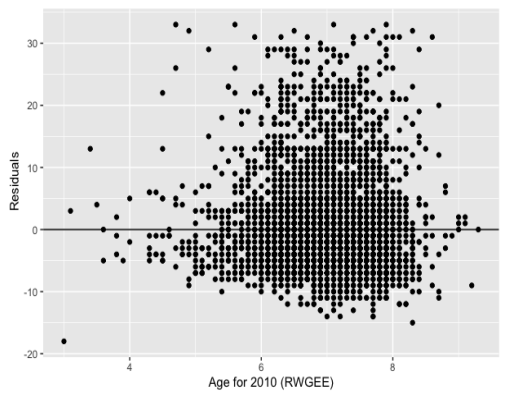

(a) WRSGEE

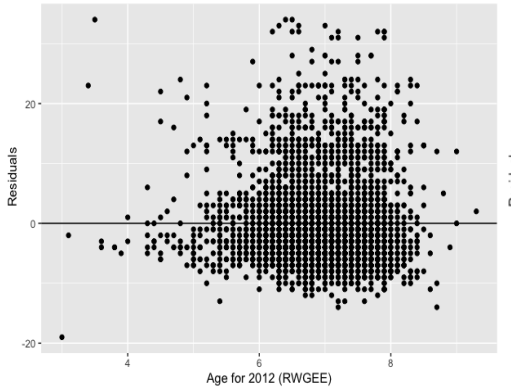

(d) WRSGEE

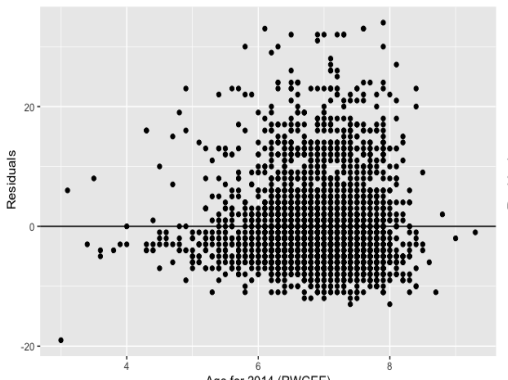

(g) WRSGEE

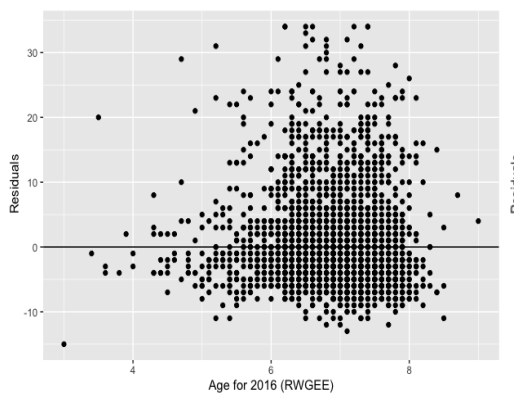

(j) WRSGEE

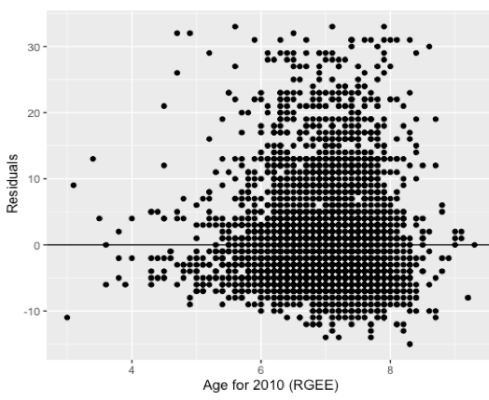

(b) RSGEE

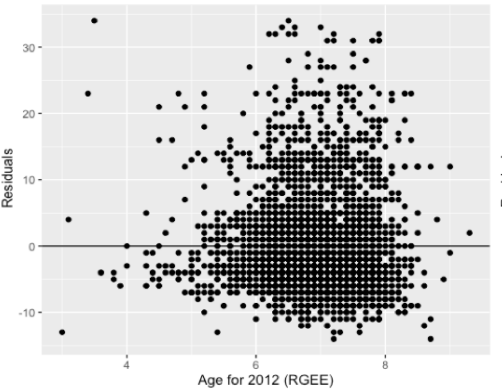

(e) RSGEE

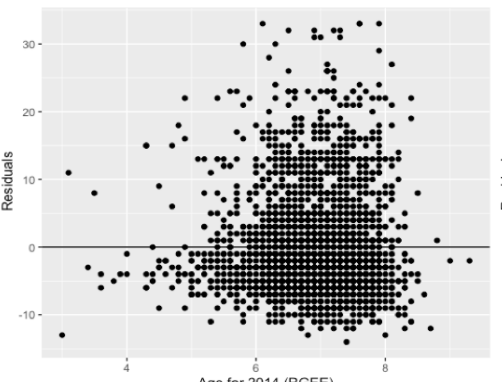

(h) RSGEE

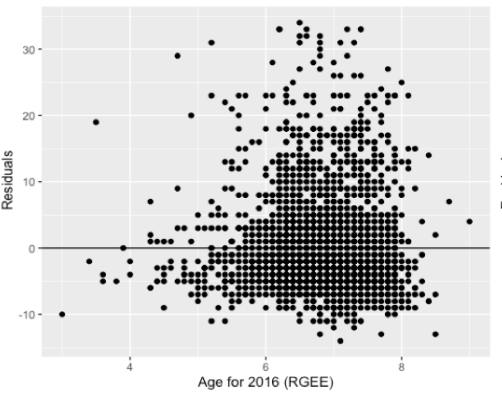

(k) RSGEE

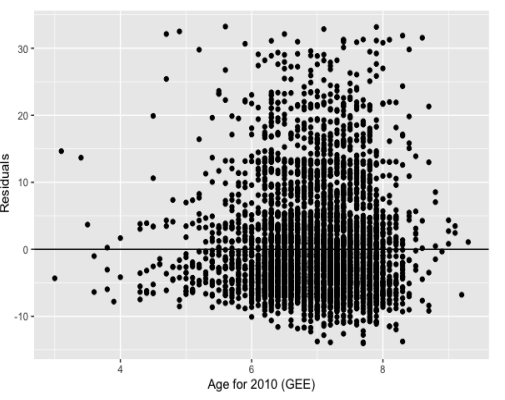

(c) GEE

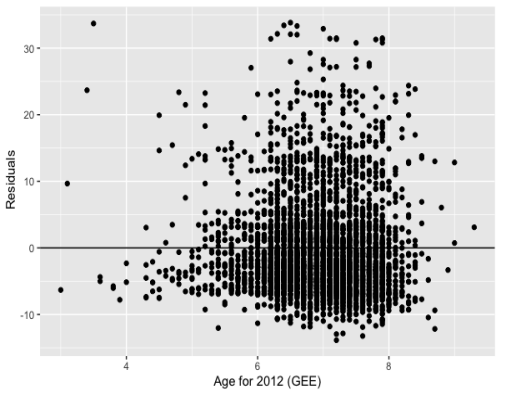

(f) GEE

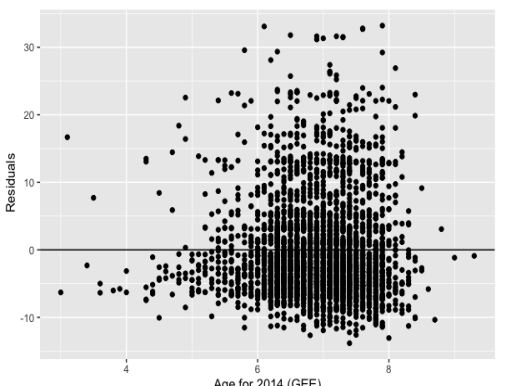

(i) GEE

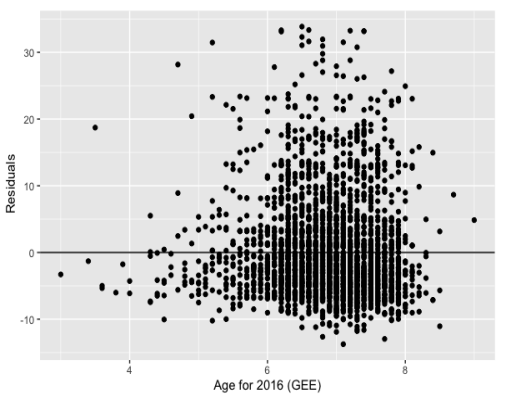

(l) GEE

Figure 6.3: Residual plots form HRS data analysis 


\subsubsection{Results}

The pseudo maximum likelihood estimates of the missing data model parameters $\boldsymbol{\tau}$, their standard errors and the corresponding z-values are presented in Table 6.2. It shows that the rates of dropout differ across all covariates as well as the number of previous doctor visits over the last 2 years. The dropout probability increases with the increased number of previous doctor visits. The study also shows that older individuals are more likely to miss a visit as compared to the younger ones. Respondents with any of the diseases (Cancer, Heart, Lung) are more likely to drop out relative to those without such diseases. Smokers are less likely to dropout as compared to non-smokers, but the effect of smoker is not significant. We also observe that time has a positive effect on the missingness of the data.

Figure 6.2 displays the estimated nonlinear functions $\hat{m}($.$) from RSGEE and$ WRSGEE methods. Here $\hat{m}($.$) shows evidence of nonlinear trends under both meth-$ ods. The WRSGEE method gives a smoother curve as compared to the unweighted partially linear method (RSGEE). Our results suggest that the data are not well fitted by the ordinary GEE method. Inclusion of the nonlinear function $m($.$) in$ (6.1) allows the model to be capture any curvature.

Figure 6.3 displays the plots of residuals vs. age in different waves under WRSGEE, RSGEE, and GEE. All residual plots display a fairly random pattern, as the residuals are distributed pretty symmetrically around the line 0 .

Table 6.3 presents the estimates, their corresponding standard errors, and zvalues for all regression, association and dispersion parameters of the marginal mean response model (6.1), as obtained by the three methods. For the linear part, the proposed WRSGEE method appears to give slightly different estimates than those obtained by the other two methods. For example, when estimating the effect of cancer, the WSPGEE method give a value of 0.156, whereas the GEE and RSGEE method give value of 0.143 and 0.140 , respectively. Also, when estimating the effect of Time, both GEE and RSGEE provide negligible values of -0.002, whereas the proposed WRSGEE method provides a significant estimates of -0.024 . We conclude 
from the results in Table 6.3 that the presence of any of the health conditions, Lung, Hosp, Heart, or Cancer, increases the number of doctor visits by respondents. Also, males make fewer doctor visits, as compared to females. The correlation parameters $\alpha$ and dispersion parameter $\phi$ appears to be significant by all methods.

Table 6.2: ML estimates, standard errors and z-value of missing data model parameters for the HRS data analysis

\begin{tabular}{rrrr}
\hline \hline & Estimate & S.E. & z-value \\
\hline Intercept & -6.4210 & 0.34886 & -18.41 \\
Previous visits & 0.0285 & 0.00326 & 8.76 \\
Age & 0.5064 & 0.04142 & 12.23 \\
Cancer & 0.1413 & 0.06412 & 2.20 \\
Heart & 0.2650 & 0.05521 & 4.80 \\
Lung & 0.5431 & 0.07077 & 7.67 \\
Smoke & -0.0657 & 0.05187 & -1.27 \\
White & -0.1530 & 0.15261 & -1.00 \\
Black & 0.2665 & 0.16915 & 1.58 \\
Time & 0.3056 & 0.03036 & 10.07 \\
\hline
\end{tabular}




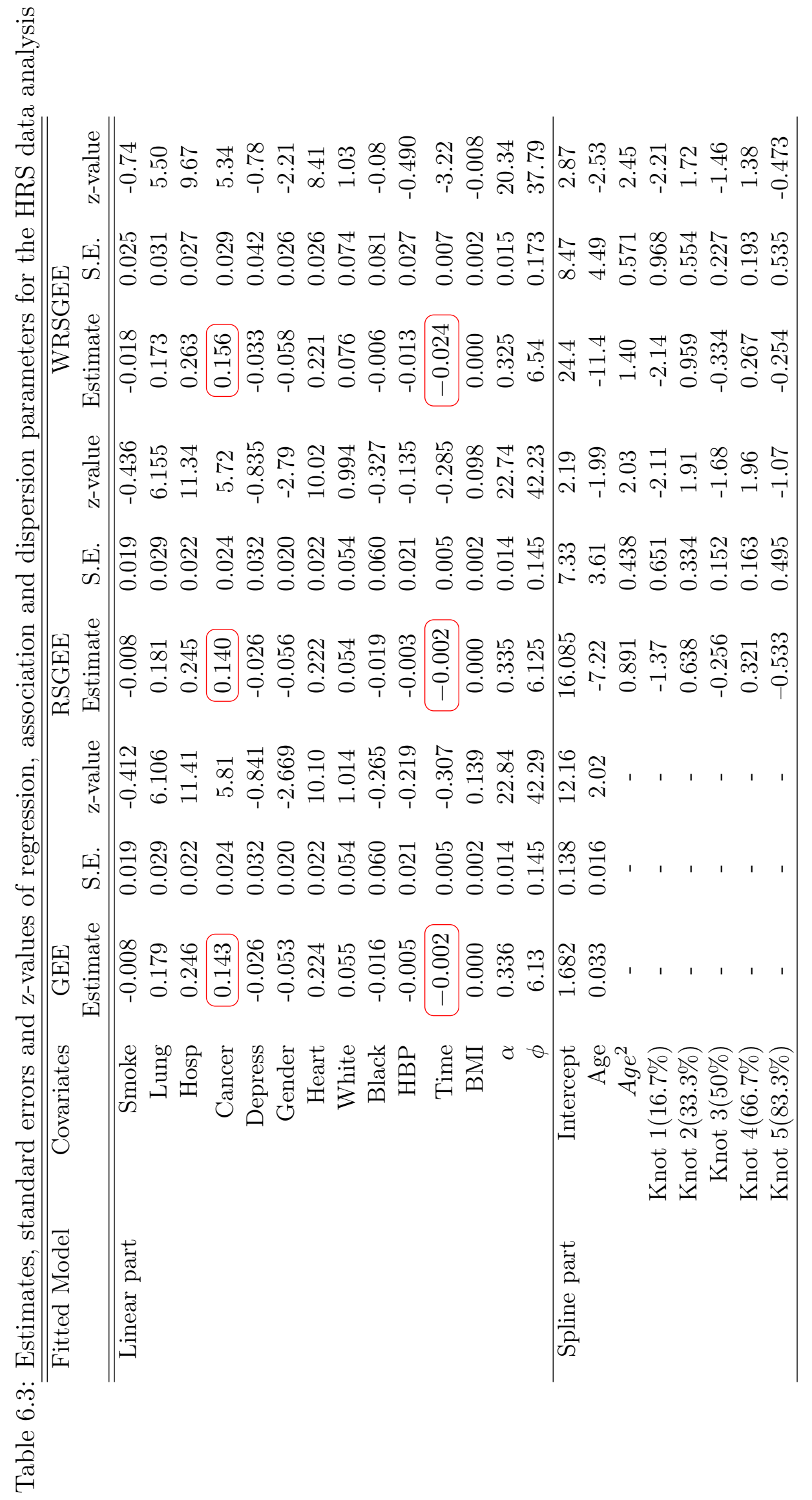


Table 6.4: Estimates of nonlinear function $m(a g e)$ for different values of age

\begin{tabular}{ccccc}
\hline \hline & & GEE & RSGEE & WRSGEE \\
& Age & $\hat{m}$ (Age) & $\hat{m}$ (Age) & $\hat{m}$ (Age) \\
\hline \hline Nonlinear function: & 4.1 & 2.06 & 1.49 & 1.31 \\
& 5.4 & 1.86 & 1.93 & 1.98 \\
& 6.2 & 1.89 & 1.87 & 1.89 \\
7.0 & 1.91 & 1.93 & 1.96 \\
8.0 & 1.95 & 1.96 & 1.97 \\
8.6 & 1.97 & 2.12 & 2.08 \\
\hline
\end{tabular}




\section{Chapter 7}

\section{Conclusion}

In this thesis, we have introduced and discussed semiparametric marginal models for analyzing incomplete binary and count longitudinal data with dropouts. In the case of binary data, we explicitly considered joint estimation of the marginal mean and association parameters, while in the case of count data, we considered joint estimation of the marginal mean, association and overdispersion parameters by using generalized second-order estimating equations when the marginal model is partially linear.

We have developed a set of weighted generalized estimating equations for fitting regression models to binary and count longitudinal responses. In the case of dropouts, when evaluating longitudinal responses, we incorporate the inverse probability weights of Robins et al. (1995) into Prentice (1988) GEE method. Our simulation study shows that the proposed WGEE2 method typically offers impartial and effective estimators if a MAR model implements the missing data mechanism. Our analysis of longitudinal data on patterns in cigarette smoking shows that this approach strengthens parameter estimates for both regression and association functions.

We also developed a semiparametric approach based on partially linear models to analyze longitudinal data. We implemented the regression spline smoothing technique to estimate the nonlinear function in the partially linear model. Our 
simulation study shows that if the true underlying model is partially linear, then our proposed method offers unbiased and efficient estimators, in general. From simulations and real data analysis, we have observed that even if a nonparametric function is used to fit the data, where the marginal model is actually represented by an ordinary generalized linear model, the proposed method still leads to unbiased estimates, although there may be some loss of efficiency. The proposed method, for the mentioned purpose, is very robust and has implementations in a wide range of settings.

Lastly, we suggested a weighted second-order GEE method to conduct a simultaneous estimation of $m(),. \boldsymbol{\beta}, \boldsymbol{\alpha}$ and $\phi$ for binary and count longitudinal dropout data. We used the proposed methods for analyzing survey data from the Health and Retirement Study (HRS)(HRS, 2019). The estimated smoothing function showed strong evidence of a nonlinear pattern. This application suggests that our proposed approach can be used to boost estimates of model parameters where data are not well suited for binary and count longitudinal responses with dropouts by an ordinary GEE model.

\subsection{Future Research}

Only monotonous missingness where subjects drop out before the end of the study and do not return are considered in this study. In practice, missing data patterns are rarely monotonous but they are close to monotonous (Little and Rubin, 1987). Future research is suggested for exploring and evaluating patterns of nonmonotone missingness in the data.

Also, the regression spline smoothing technique is used to estimate a single nonlinear function in this study. The suggested spline-based approach can easily be extended to include generalizations of partially linear models that produce a partially linear additive model. 


\section{Bibliography}

Bahadur, R. (1961). A representation of the joint distribution of responses to $n$ dichotomous items. Standford mathematical studies in the Social Sciences VI, pages $158-168$.

Baker, S. G. and Laird, N. M. (1988). Regression analysis for categorical variables with outcome subject to nonignorable nonresponse. Journal of the American Statistical association, 83(401):62-69.

Carey, V., Zeger, S. L., and Diggle, P. (1993). Modelling multivariate binary data with alternating logistic regressions. Biometrika, 80(3):517-526.

Carroll, R. J., Fan, J., Gijbels, I., and Wand, M. P. (1997). Generalized partially linear single-index models. Journal of the American Statistical Association, 92(438):477-489.

Claeskens, G., Krivobokova, T., and Opsomer, J. D. (2009). Asymptotic properties of penalized spline estimators. Biometrika, 96(3):529-544.

Cui, J. and Qian, G. (2007). Selection of working correlation structure and best model in gee analyses of longitudinal data. Communications in Statistics-Simulation and Computation $\AA, 36(5): 987-996$.

De Boor, C. (1978). A Practical Guide to Splines, volume 27. Applied Mathematical Sciences, New York: Springer-Verlag.

Diggle, P. J., Heagerty, P., Liang, K.-Y., and Zeger, S. L. (2002). Analysis of Longitudinal Data, 2nd ed. New York: Oxford University Press. 
Ekholm, A. and Skinner, C. (1998). The muscatine children's obesity data reanalysed using pattern mixture models. Journal of the Royal Statistical Society: Series C (Applied Statistics), 47(2):251-263.

Eubank, R. L. (1999). Nonparametric Regression and Spline Smoothing, 2nd ed. New York: CRC press.

Fan, J. and Li, R. (2004). New estimation and model selection procedures for semiparametric modeling in longitudinal data analysis. Journal of the American Statistical Association, 99(467):710-723.

Fitzmaurice, G., Davidian, M., Verbeke, G., and Molenberghs, G. (2008). Longitudinal Data Analysis. New York: CRC press.

Fitzmaurice, G. M., Laird, N. M., and Ware, J. H. (2012). Applied Longitudinal Analysis, 2nd ed., volume 998. New Jersy: John Wiley \& Sons.

Fitzmaurice, G. M., Molenberghs, G., and Lipsitz, S. R. (1995). Regression models for longitudinal binary responses with informative drop-outs. Journal of the Royal Statistical Society: Series B (Methodological), 57(4):691-704.

Friedman, J. H. et al. (1991). Multivariate adaptive regression splines. The annals of statistics, 19(1):1-67.

Friedman, J. H. and Silverman, B. W. (1989). Flexible parsimonious smoothing and additive modeling. Technometrics, 31(1):3-21.

Grace, Y. Y., He, W., and Liang, H. (2009). Semiparametric marginal and association regression methods for clustered binary data. Annals of the Institute of Statistical Mathematics, 100(2):278.

Hall, D. B. and Severini, T. A. (1998). Extended generalized estimating equations for clustered data. Journal of the American Statistical Association, 93(444):13651375. 
He, X., Fung, W. K., and Zhu, Z. (2005). Robust estimation in generalized partial linear models for clustered data. Journal of the American Statistical Association, 100(472):1176-1184.

He, X. and Shi, P. (1996). Bivariate tensor-product b-splines in a partly linear model. Journal of Multivariate Analysis, 58(2):162-181.

He, X., Zhu, Z.-Y., and Fung, W.-K. (2002). Estimation in a semiparametric model for longitudinal data with unspecified dependence structure. Biometrika, 89(3):579-590.

Heckman, N. E. (1986). Spline smoothing in a partly linear model. Journal of the Royal Statistical Society: Series B (Methodological), 48(2):244-248.

HRS (2019). Health and Retirement Study, (RAND HRS Longitudinal File 2016) public use dataset. Produced and distributed by the University of Michigan with funding from the National Institute on Aging (grant number NIA U01AG009740). Ann Arbor, MI.

Huang, G.-H., Bandeen-Roche, K., and Rubin, G. S. (2002). Building marginal models for multiple ordinal measurements. Journal of the Royal Statistical Society: Series C (Applied Statistics), 51(1):37-57.

Huang, J. Z., Zhang, L., and Zhou, L. (2007). Efficient estimation in marginal partially linear models for longitudinal/clustered data using splines. Scandinavian Journal of Statistics, 34(3):451-477.

Huggins, R. and Loesch, D. (1998). On the analysis of mixed longitudinal growth data. Biometrics, pages 583-595.

Hughes, G. H., Cutter, G., Donahue, R., Friedman, G. D., Hulley, S., Hunkeler, E., Jacobs Jr, D. R., Liu, K., Orden, S., Pirie, P., et al. (1987). Recruitment in the coronary artery disease risk development in young adults (cardia) study. Controlled clinical trials, 8(4):68-73. 
Kauermann, G., Krivobokova, T., and Fahrmeir, L. (2009). Some asymptotic results on generalized penalized spline smoothing. Journal of the Royal Statistical Society: Series B (Statistical Methodology), 71(2):487-503.

Leng, C., Zhang, W., and Pan, J. (2010). Semiparametric mean-covariance regression analysis for longitudinal data. Journal of the American Statistical Association, 105(489):181-193.

Liang, K.-Y. and Zeger, S. L. (1986). Longitudinal data analysis using generalized linear models. Biometrika, 73(1):13-22.

Lin, D. and Ying, Z. (2001). Semiparametric and nonparametric regression analysis of longitudinal data. Journal of the American Statistical Association, 96(453):103126.

Lin, H., Fu, B., Qin, G., and Zhu, Z. (2017). Doubly robust estimation of generalized partial linear models for longitudinal data with dropouts. Biometrics, 73(4):11321139.

Lin, X. and Carroll, R. J. (2000). Nonparametric function estimation for clustered data when the predictor is measured without/with error. Journal of the American statistical Association, 95(450):520-534.

Lin, X. and Carroll, R. J. (2001a). Semiparametric regression for clustered data. Biometrika, 88(4):1179-1185.

Lin, X. and Carroll, R. J. (2001b). Semiparametric regression for clustered data using generalized estimating equations. Journal of the American Statistical Association, 96(455):1045-1056.

Lin, X., Wang, N., Welsh, A. H., and Carroll, R. J. (2004). Equivalent kernels of smoothing splines in nonparametric regression for clustered/longitudinal data. Biometrika, 91(1):177-193. 
Lipsitz, S. R., Laird, N. M., and Harrington, D. P. (1991). Generalized estimating equations for correlated binary data: Using the odds ratio as a measure of association. Biometrika, 78(1):153-160.

Little, R. J. and Rubin, D. B. (1987). Statistical Analysis With Missing Data, volume 793. New York: John Wiley \& Sons.

Martinussen, T. and Scheike, T. H. (2002). A flexible additive multiplicative hazard model. Biometrika, 89(2):283-298.

Mirelesh, R. and Leppik, I. E. (1985). Valproate and clonazepam comedication in patients with intractable epilepsy. Epilepsia, 26(2):122-126.

Molinari, N., Durand, J.-F., and Sabatier, R. (2004). Bounded optimal knots for regression splines. Computational statistics $\&$ data analysis, 45(2):159-178.

Pepe, M. S. and Couper, D. (1997). Modeling partly conditional means with longitudinal data. Journal of the American Statistical Association, 92(439):991-998.

Preisser, J. S., Lohman, K. K., and Rathouz, P. J. (2002). Performance of weighted estimating equations for longitudinal binary data with drop-outs missing at random. Statistics in medicine, 21(20):3035-3054.

Prentice, R. L. (1988). Correlated binary regression with covariates specific to each binary observation. Biometrics, pages 1033-1048.

Qin, G., Mao, J., and Zhu, Z. (2016). Joint mean-covariance model in generalized partially linear varying coefficient models for longitudinal data. Journal of Statistical Computation and Simulation, 86(6):1166-1182.

RAND HRS (2019). RAND HRS Longitudinal File 2016. Produced by the RAND Center for the Study of Aging, with funding from the National Institute on Aging and the Social Security Administration. Santa Monica, CA.

Rice, J. (1986). Convergence rates for partially splined models. Statistics \& probability letters, 4(4):203-208. 
Robins, J. M., Rotnitzky, A., and Zhao, L. P. (1995). Analysis of semiparametric regression models for repeated outcomes in the presence of missing data. Journal of the american statistical association, 90(429):106-121.

Rubin, D. B. (1976). Inference and missing data. Biometrika, 63(3):581-592.

Ruppert, D. and Carroll, R. J. (2000). Theory methods: Spatially-adaptive penalties for spline fitting. Australian 83 New Zealand Journal of Statistics, 42(2):205223.

Severini, T. A. and Staniswalis, J. G. (1994). Quasi-likelihood estimation in semiparametric models. Journal of the American statistical Association, 89(426):501511.

Shi, M., Weiss, R. E., and Taylor, J. M. (1996). An analysis of paediatric cd4 counts for acquired immune deficiency syndrome using flexible random curves. Journal of the Royal Statistical Society: Series C (Applied Statistics), 45(2):151-163.

Smith, M. and Kohn, R. (1996). Nonparametric regression using bayesian variable selection. Journal of Econometrics, 75(2):317-343.

Smith, P. L. (1979). Splines as a useful and convenient statistical tool. The American Statistician, 33(2):57-62.

Snedecor, G. W. and Cochran, W. G. (1967). Statistical Methods, 6th ed. Ames, Iowa: Iowa State University Press.

Speckman, P. (1988). Kernel smoothing in partial linear models. Journal of the Royal Statistical Society: Series B (Methodological), 50(3):413-436.

Stone, C. J. (1985). Additive regression and other nonparametric models. The annals of Statistics, pages 689-705.

Sullivan Pepe, M. and Anderson, G. L. (1994). A cautionary note on inference for marginal regression models with longitudinal data and general correlated response data. Communications in statistics-simulation and computation, 23(4):939-951. 
Thall, P. F. and Vail, S. C. (1990). Some covariance models for longitudinal count data with overdispersion. Biometrics, pages 657-671.

Wang, N. (2003). Marginal nonparametric kernel regression accounting for withinsubject correlation. Biometrika, 90(1):43-52.

Wang, N., Carroll, R. J., and Lin, X. (2005). Efficient semiparametric marginal estimation for longitudinal/clustered data. Journal of the American Statistical Association, 100(469):147-157.

Wang, Y. (1998). Mixed effects smoothing spline analysis of variance. Journal of the royal statistical society: Series b (statistical methodology), 60(1):159-174.

White, H. (1982). Maximum likelihood estimation of misspecified models. Econometrica: Journal of the Econometric Society, pages 1-25.

Wold, S. (1974). Spline functions in data analysis. Technometrics, 16(1):1-11.

Woolson, R. F. and Clarke, W. R. (1984). Analysis of categorical incomplete longitudinal data. Journal of the Royal Statistical Society: Series A (General), 147(1):8799.

Wu, F. and Zhang, G. (2002). Genotypic variation in kernel heavy metal concentrations in barley and as affected by soil factors. Journal of Plant Nutrition, 25(6):1163-1173.

Wu, H. and Zhang, J.-T. (2006). Nonparametric Regression Methods for Longitudinal Data Analysis: Mixed-Effects Modeling Approaches, volume 515. New York: John Wiley \& Sons.

Yi, G. Y. and Cook, R. J. (2002). Marginal methods for incomplete longitudinal data arising in clusters. Journal of the American Statistical Association, 97(460):10711080. 
Zeger, S. L. and Diggle, P. J. (1994). Semiparametric models for longitudinal data with application to cd4 cell numbers in hiv seroconverters. Biometrics, pages 689-699.

Zhang, H. (1997). Multivariate adaptive splines for analysis of longitudinal data. Journal of Computational and Graphical Statistics, 6(1):74-91.

Zhou, J., Zhu, Z., and Fung, W. K. (2008). Robust testing with generalized partial linear models for longitudinal data. Journal of Statistical Planning and Inference, 138(6):1871-1883. 


\section{Appendix A}

\section{R Codes of Simulation Study for}

\section{Correlated Binary Responses}

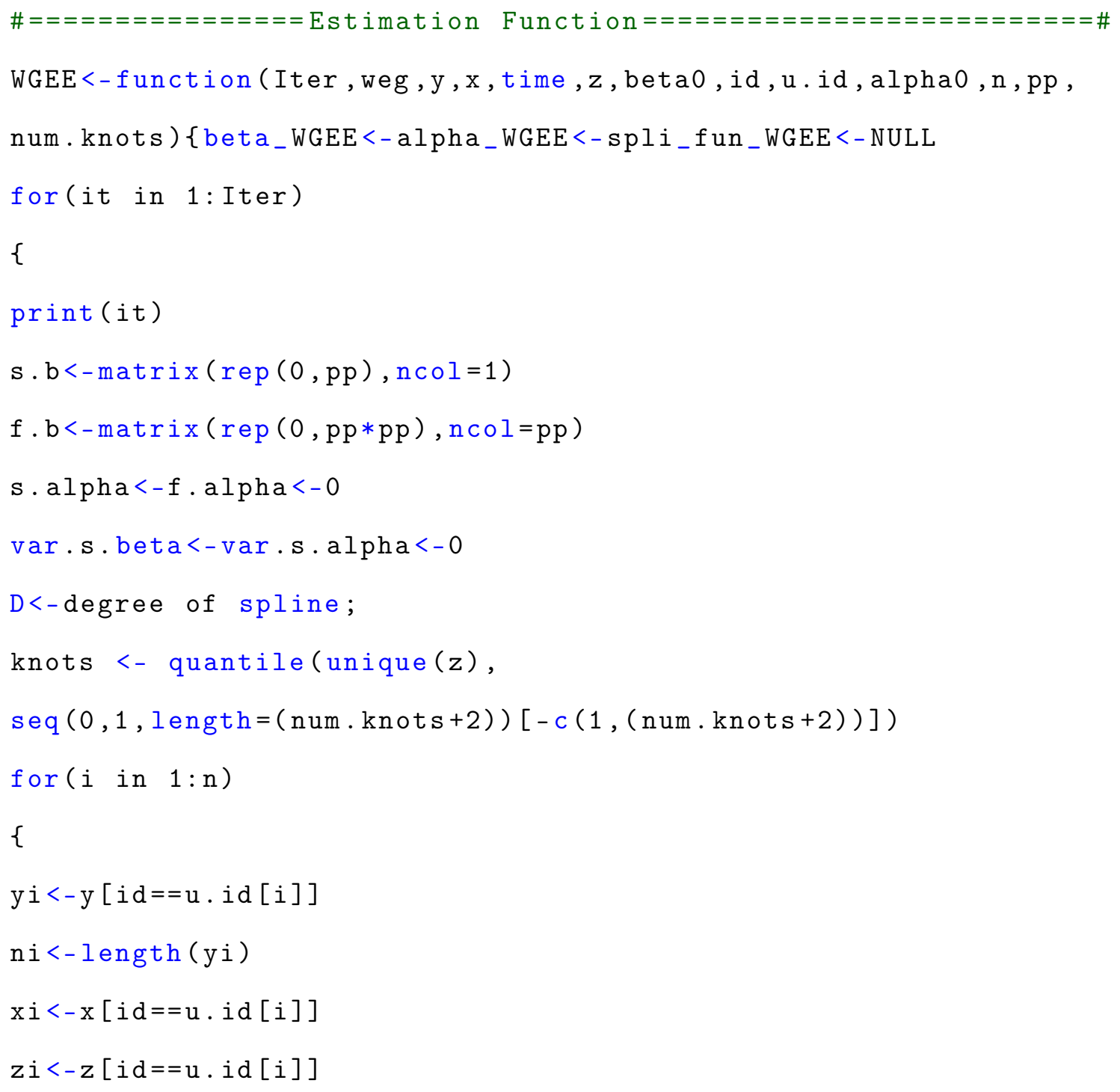




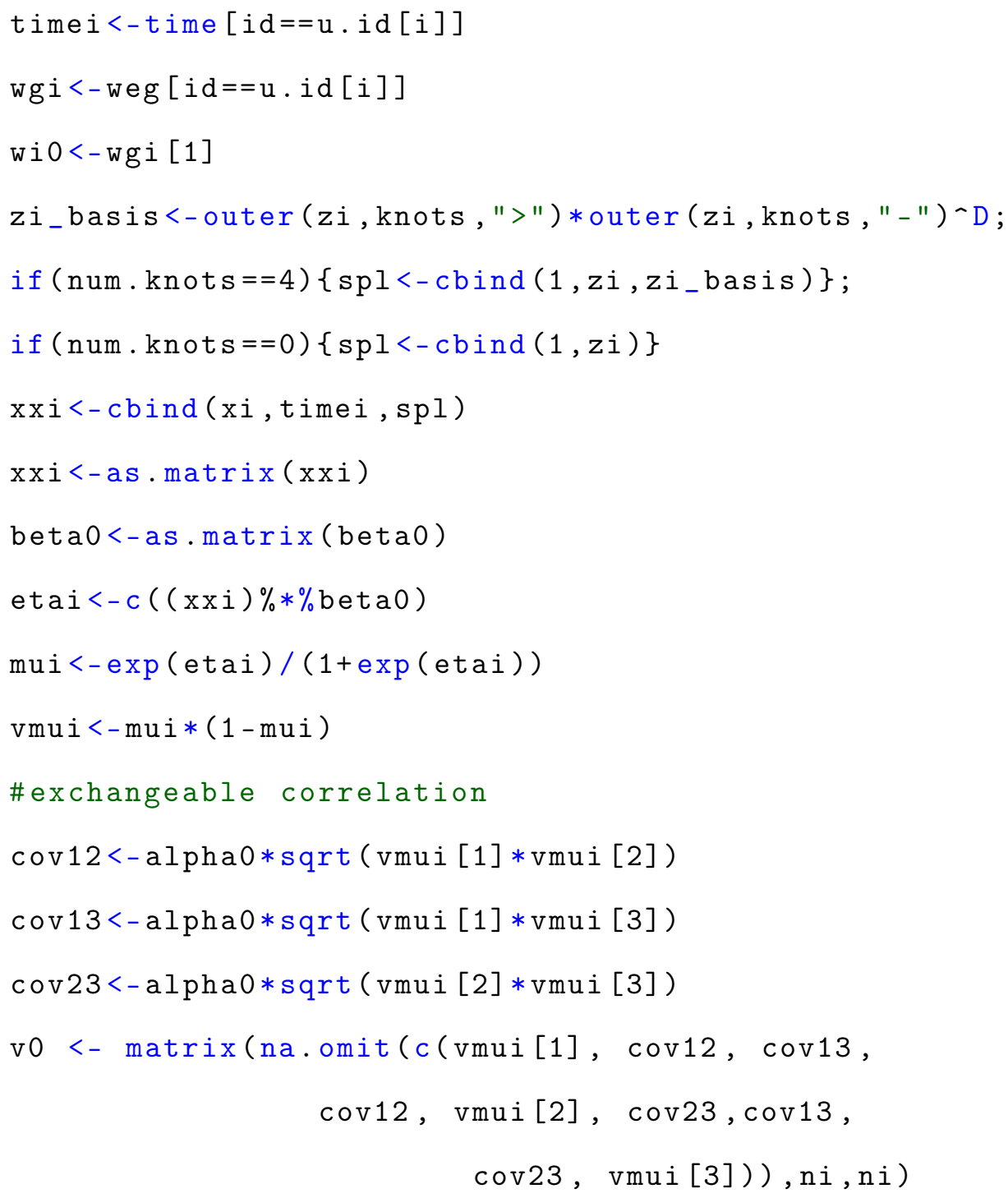

v0. inv<-solve ( $\mathrm{v} 0)$

dmui.detai<-diag (vmui, ni, ni)

dmui.dbetai<-t ( $x x i) \% * \%$ dmui.detai

score.betai<-wio* (dmui.dbetai $\% * \%$ v0.inv $\% * \%$ (yi-mui))

fisher. betai<-wi0*(dmui.dbetai $\% * \%$ v0. inv $\% * \%$ t (dmui.dbetai ) )

s. b $<-s \cdot b+$ score. betai

f. $b<-f . b+f i s h e r . b e t a i$

var.score.betai<-score.betai $\% * \%$ t (score.betai)

var.s.beta<-var.s.beta+var. score.betai

\#\#==========Estimating Alpha= $\operatorname{corr}\left(\mathrm{yij}_{-} \mathrm{i}, \mathrm{yij} \mathrm{H}_{\mathrm{k}}\right)=========\#$

$r<-(y i-m u i) / \operatorname{sqrt}(\operatorname{vmui})$

$\mathrm{w}<-\mathrm{v} . \mathrm{w}<-\mathrm{NULL}$ 


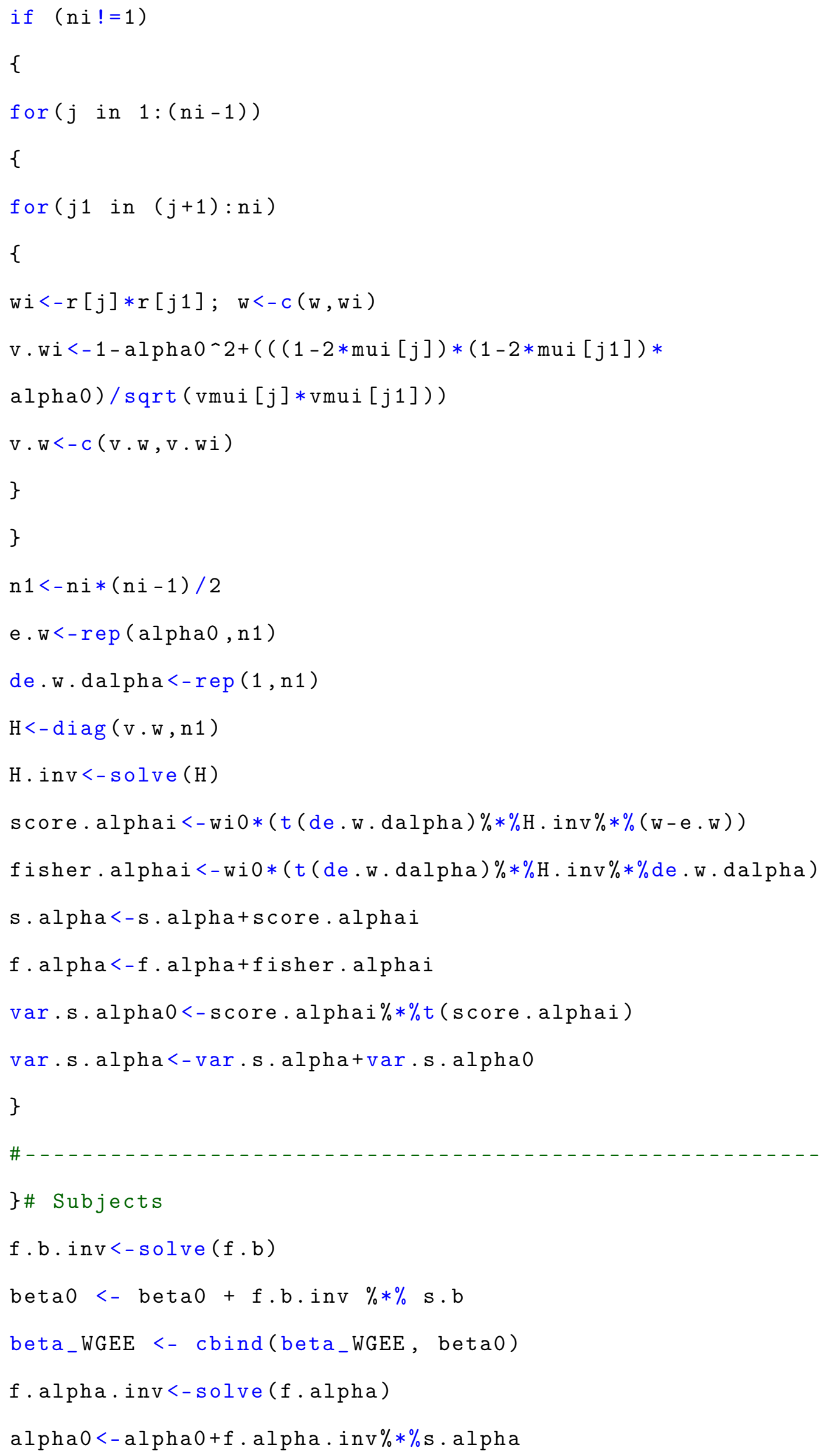




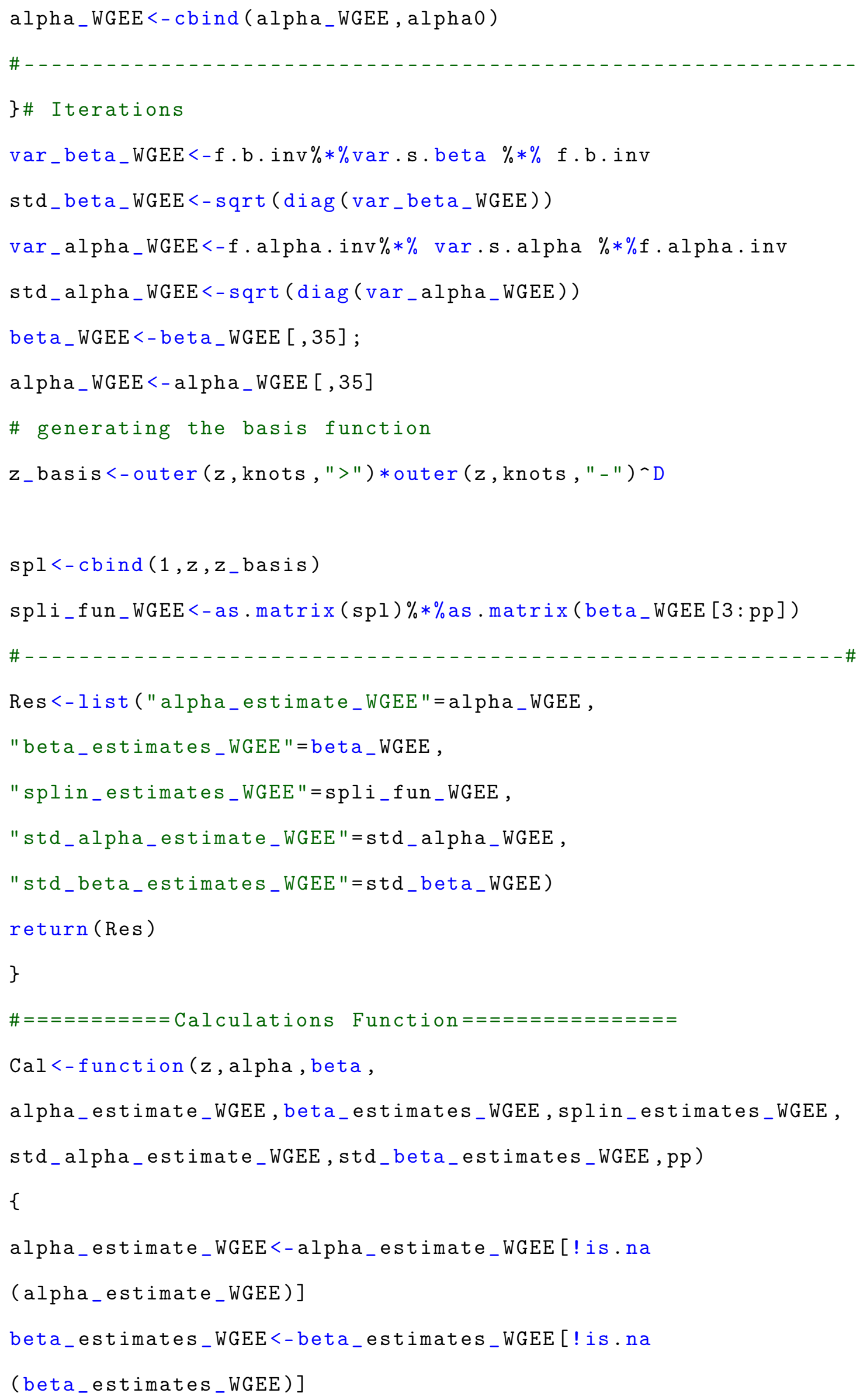




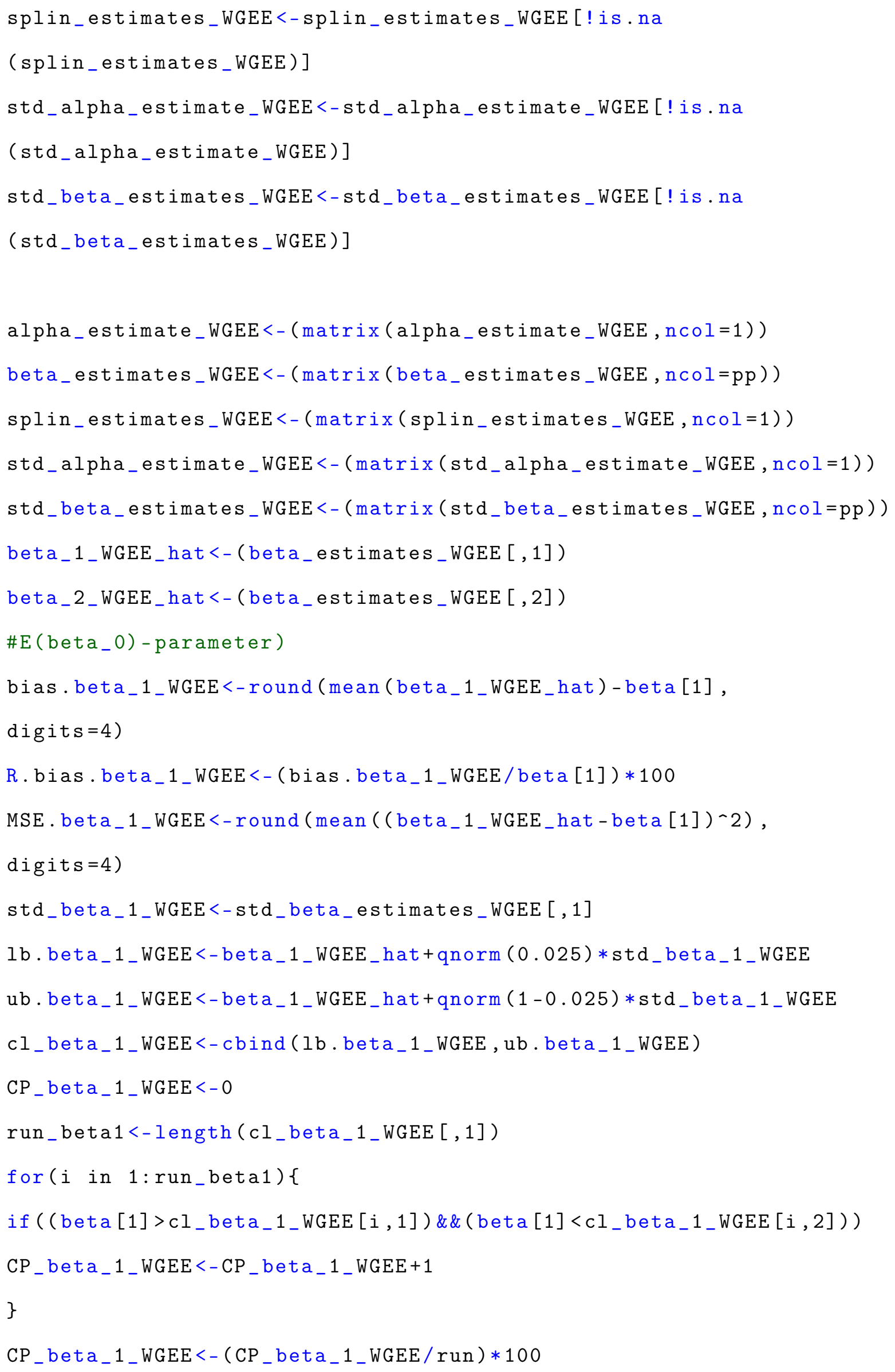


CP_beta_1_WGEE

bias.beta_2_WGEE<-round (mean (beta_2_WGEE_hat) - beta [2],

digits $=4$ )

R. bias. beta_2_WGEE<-(bias.beta_2_WGEE/beta [2] ) *100

MSE . beta_2_WGEE<-round (mean ( (beta_2_WGEE_hat-beta [2] ) -2),

digits $=4$ )

std_beta_2_WGEE<-std_beta_estimates_WGEE [, 2]

lb. beta_2_WGEE<-beta_2_WGEE_hat+qnorm $(0.025) *$ std_beta_2_WGEE

ub. beta_2_WGEE<-beta_2_WGEE_hat+qnorm $(1-0.025) *$ std_beta_2_WGEE

cl_beta_2_WGEE<-cbind ( $1 \mathrm{~b}$. beta_2_WGEE, ub. beta_2_WGEE)

length ( cl_beta_2_WGEE)

CP_beta_2_WGEE $<-0$

run_beta $2<-1$ ength $\left(\mathrm{cl}{ }_{-}\right.$beta_2_WGEE $\left.[, 1]\right)$

for ( $i$ in 1:run_beta2) \{

if ( (beta [2]>cl_beta_2_WGEE [i, 1]) \&\&(beta [2]<cl_beta_2_WGEE [i, 2] ))

CP_beta_2_WGEE<-CP_beta_2_WGEE+1

\}

CP_beta_2_WGEE<-( CP_beta_2_WGEE/run $) * 100$

bias_beta_WGEE<-c(bias.beta_1_WGEE, bias.beta_2_WGEE) \#

MSE_beta_WGEE<-c(MSE.beta_1_WGEE, MSE.beta_2_WGEE) \#

CP_beta_WGEE<-c ( CP_beta_1_WGEE, CP_beta_2_WGEE)

table_beta_WGEE_splin<-cbind(beta, bias_beta_WGEE, MSE_beta_WGEE,

CP_beta_WGEE)

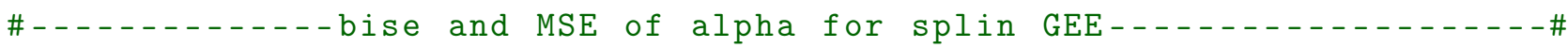

alpha_hat_WGEE<-(alpha_estimate_WGEE)

bias.alpha_WGEE<-round ( (mean (alpha_hat_WGEE)-alpha),

digits =4) \#

R.bias.alpha_WGEE<-(bias.alpha_WGEE/alpha) $* 100$

MSE.alpha_WGEE<-round (mean ( ( alpha_hat_WGEE -

matrix (rep (alpha, length (alpha_hat_WGEE)), ncol=1) )`2), digits=4)\#

std.alpha_WGEE<-std_alpha_estimate_WGEE 


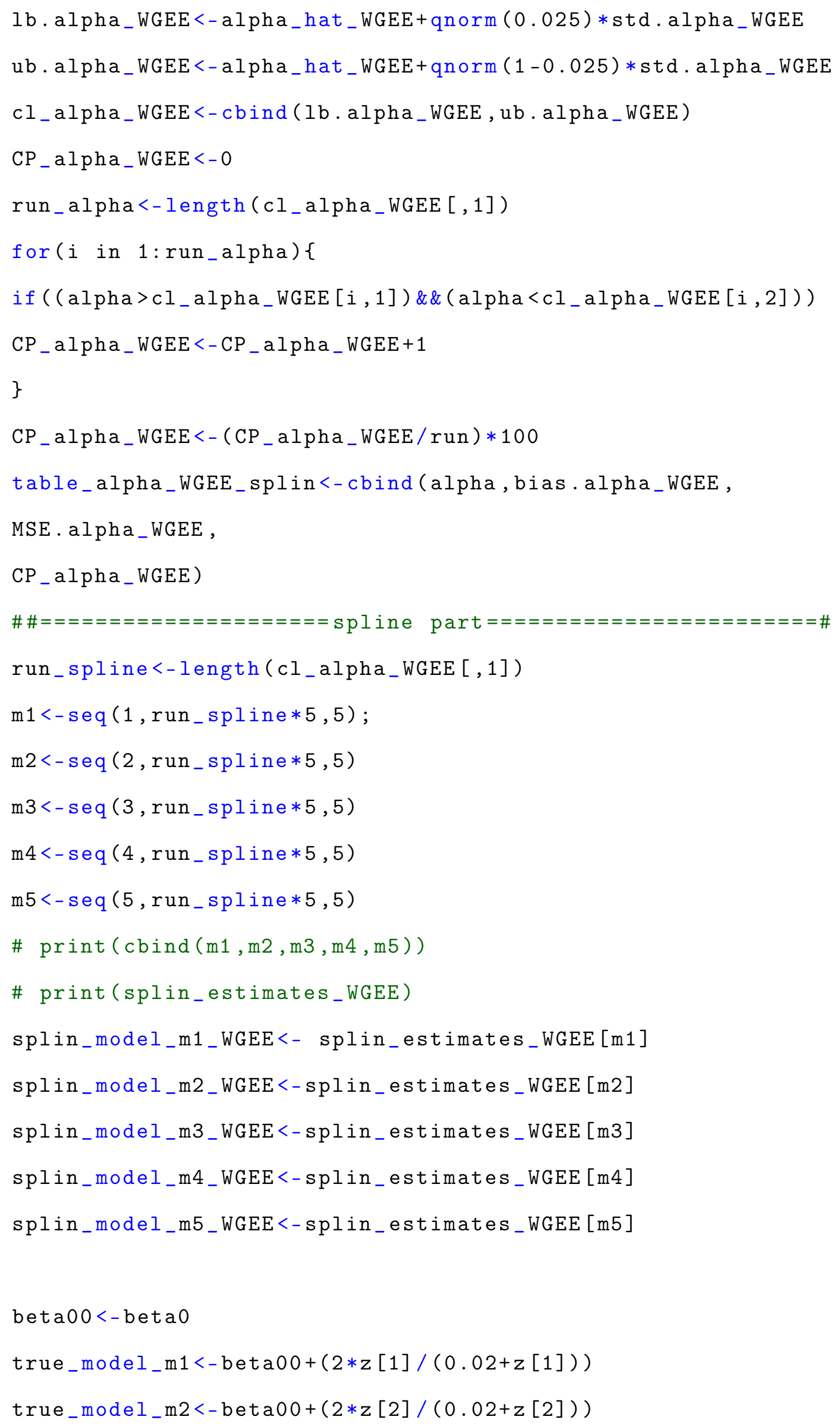




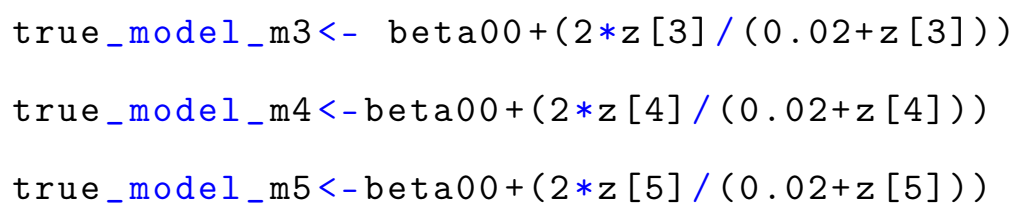




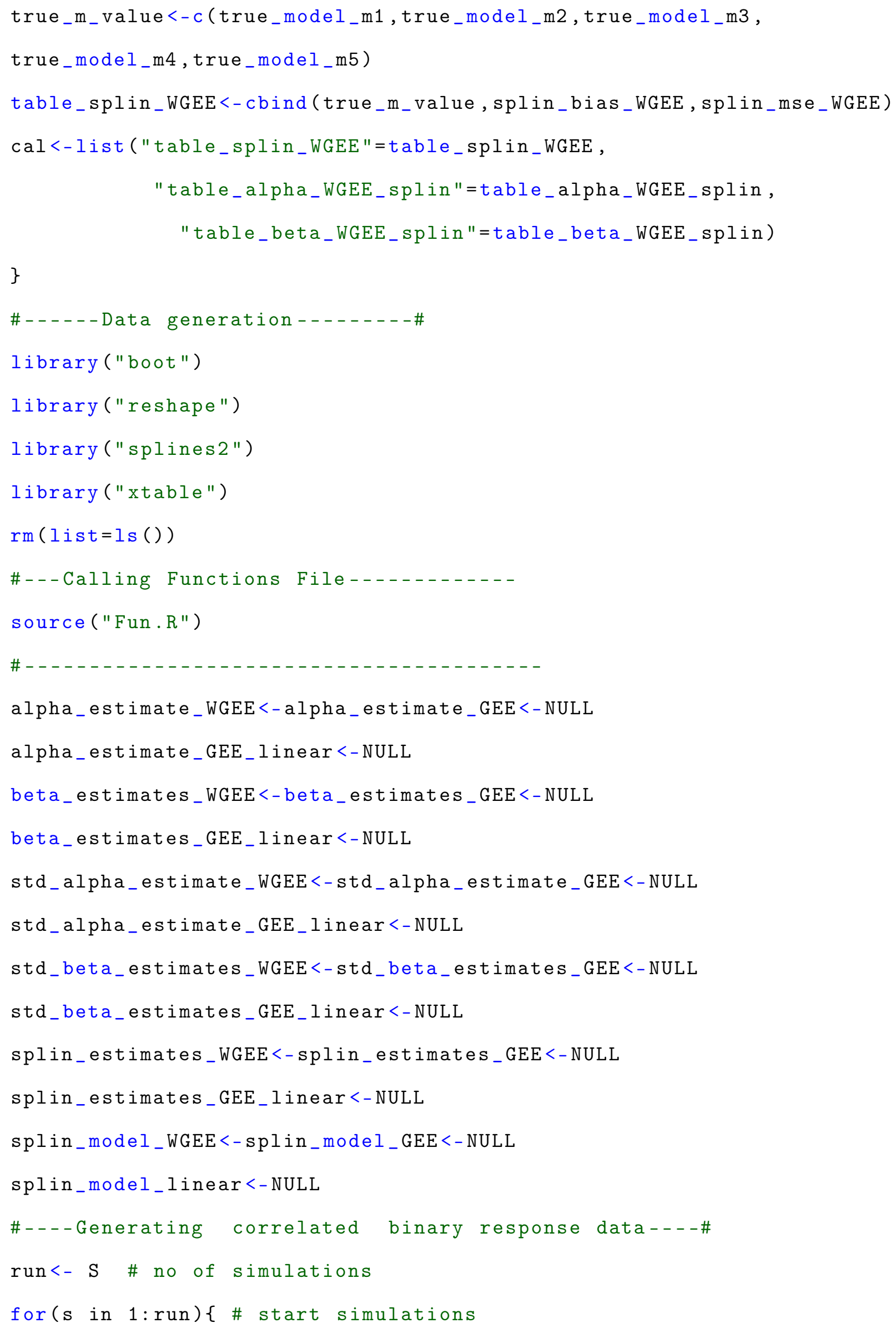




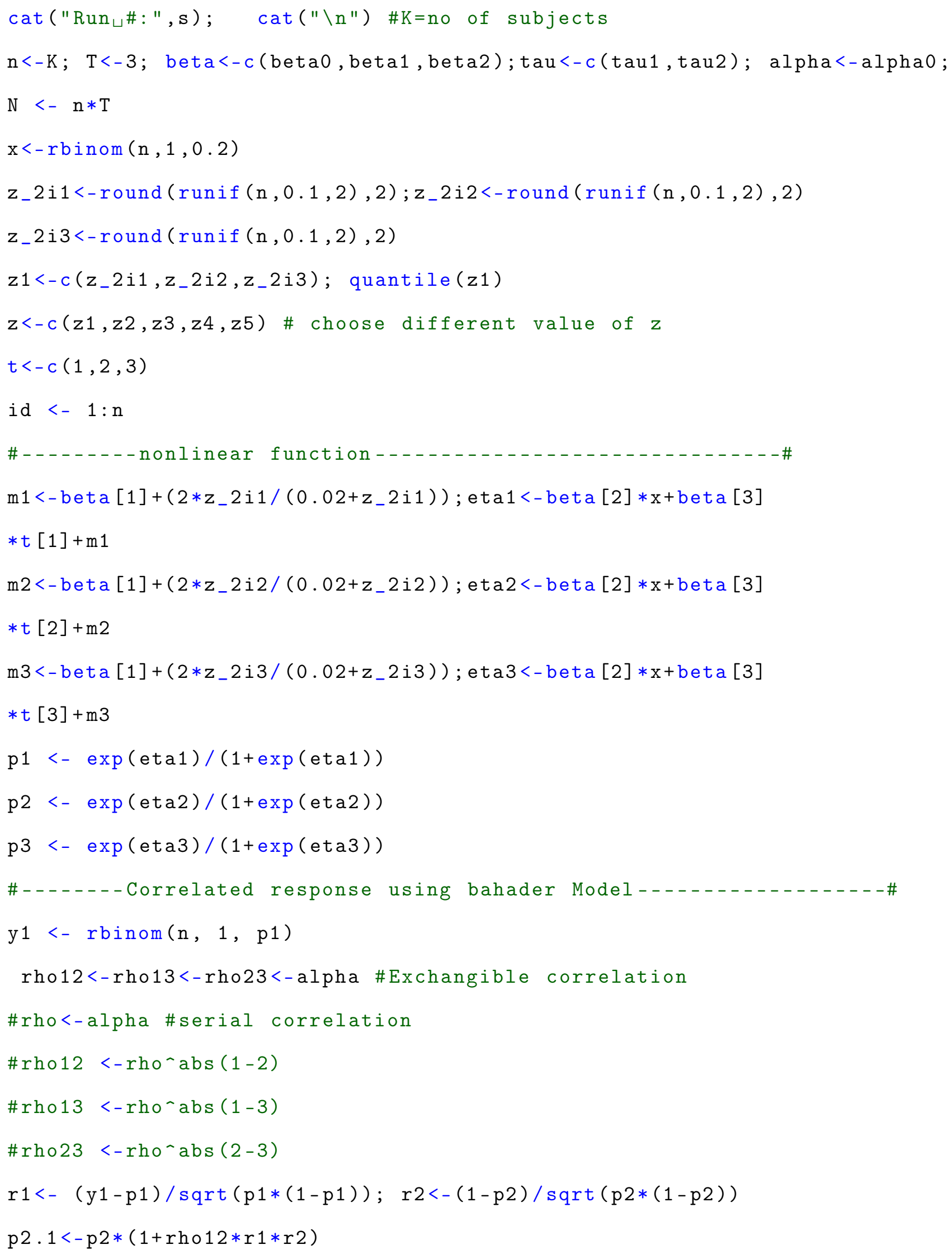




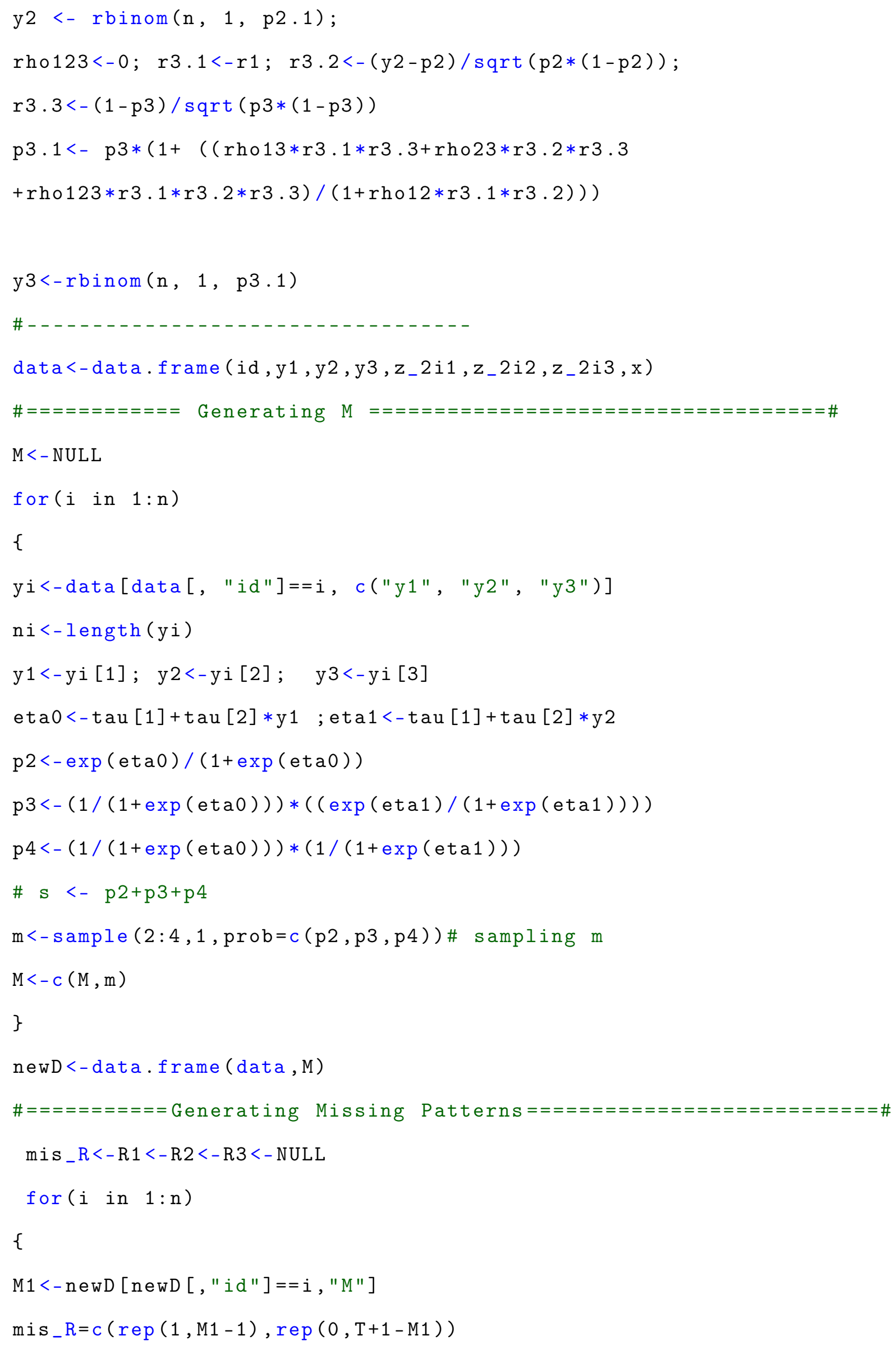




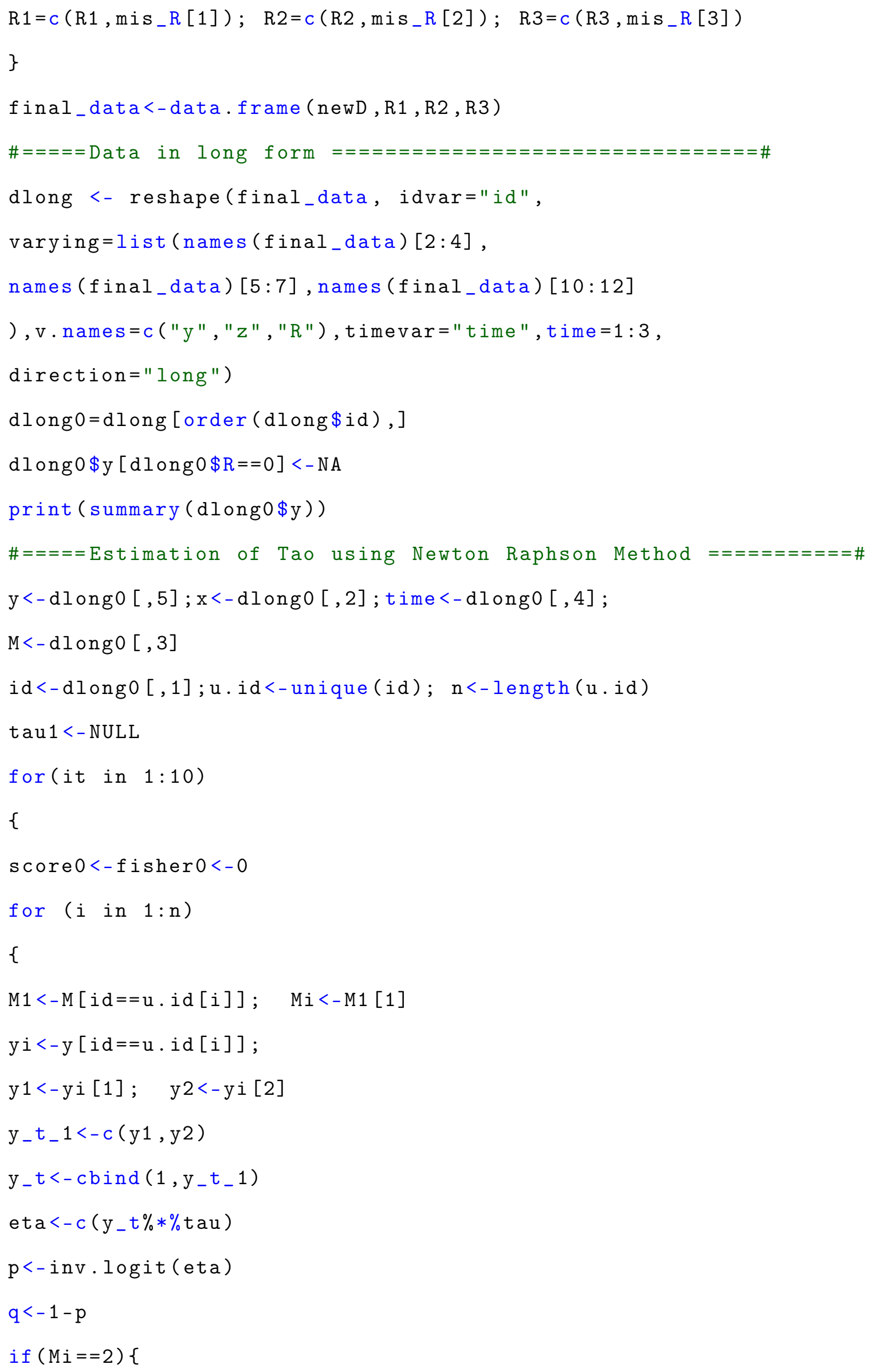




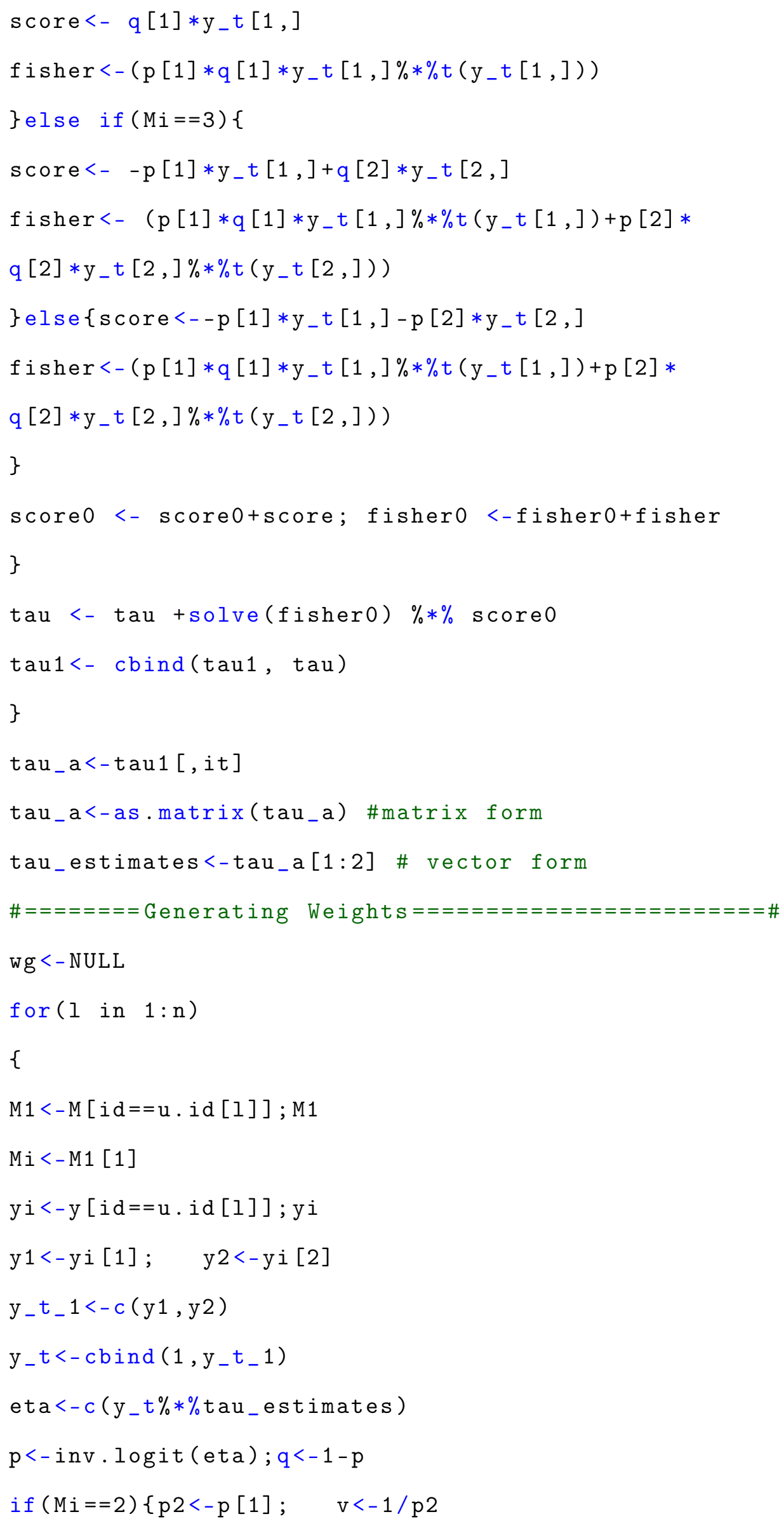




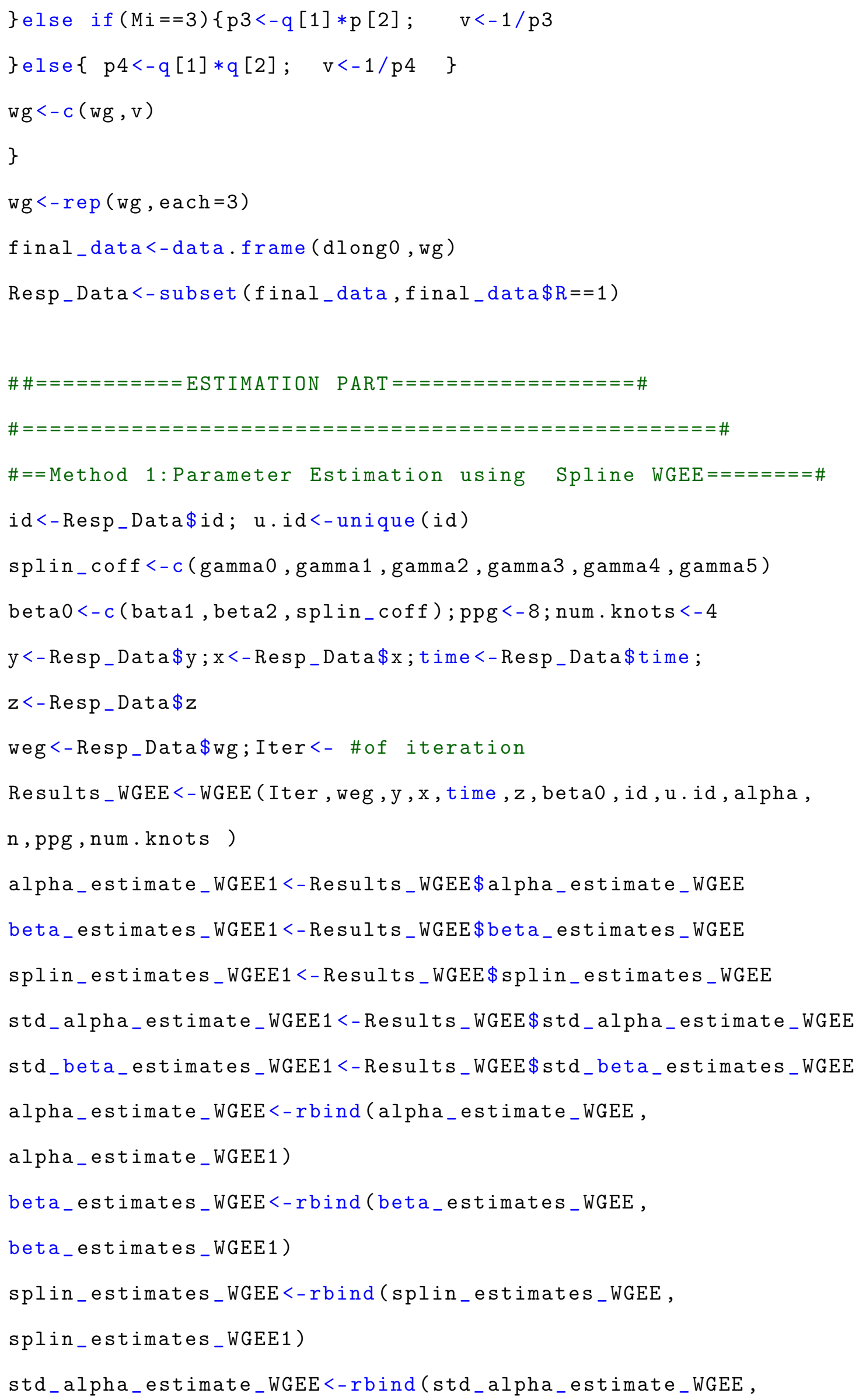




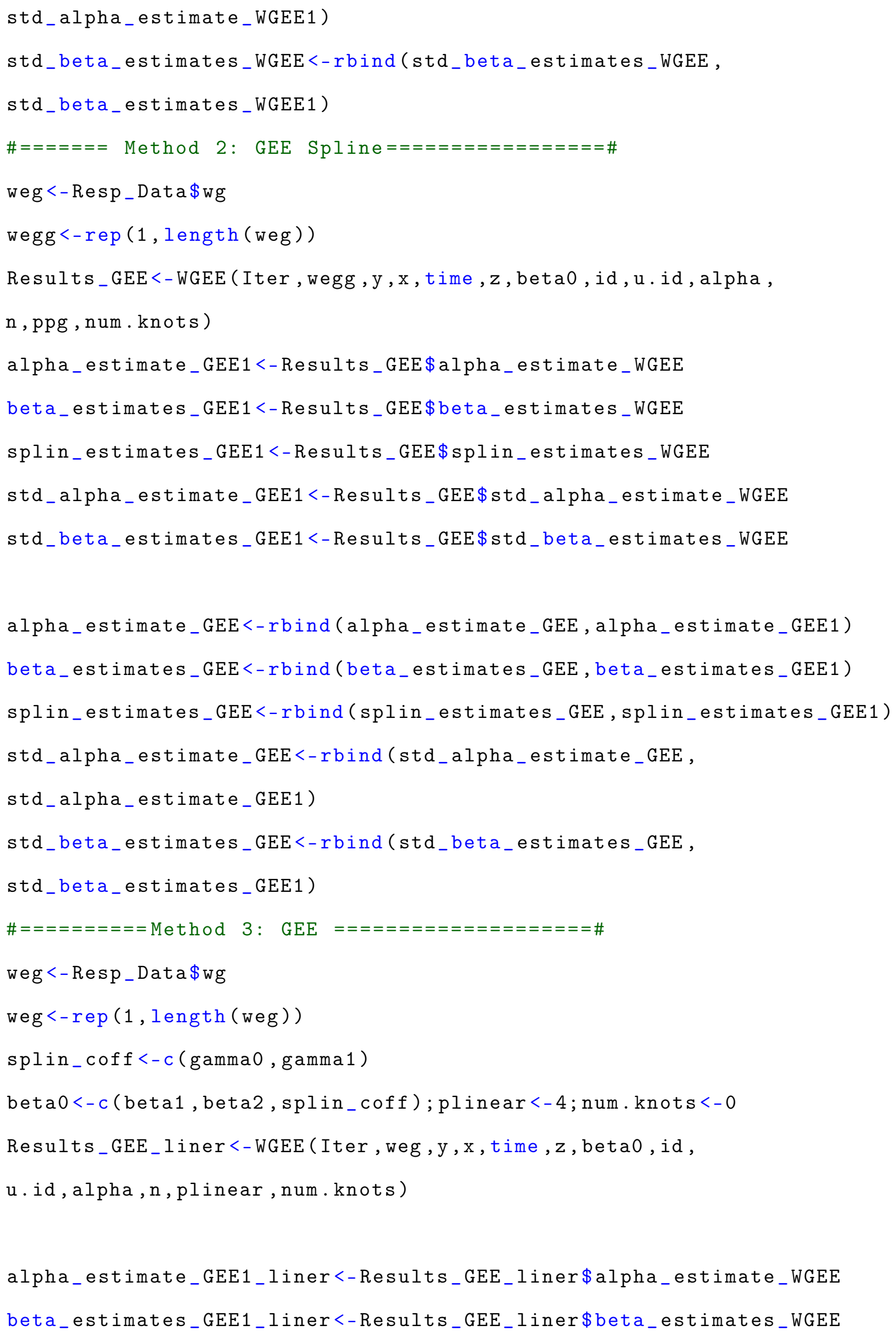


splin_estimates_GEE1_liner<-Results_GEE_liner\$splin_estimates_WGEE std_alpha_estimate_GEE1_liner<-Results_GEE_liner\$std_alpha_estimate_WGEE std_beta_estimates_GEE1_liner<-Results_GEE_liner $\$$ std_beta_estimates_WGEE

alpha_estimate_GEE_liner<-rbind (alpha_estimate_GEE_liner, alpha_estimate_GEE1_liner)

beta_estimates_GEE_liner<-rbind (beta_estimates_GEE_liner, beta_estimates_GEE1_liner) splin_estimates_GEE_liner<-rbind (splin_estimates_GEE_liner, splin_estimates_GEE1_liner) std_alpha_estimate_GEE_liner<-rbind(std_alpha_estimate_GEE_liner, std_alpha_estimate_GEE1_liner)

std_beta_estimates_GEE_liner<-rbind(std_beta_estimates_GEE_liner, std_beta_estimates_GEE1_liner)

\}

\#==-=== = = = = = C Calculations $==============\# \# \# \# \# \# \# \# \# \# \# \# \# \# \# \#$ \#-- - - - - - - - WGEE - - - - - - - - - - - - - - -

$z<-c(z 1, z 2, z 3, z 4, z 5 ;$ beta<-c(beta1, beta2); alpha<-alpha0;ppg<-total no of RR_WGEE<-Cal $(z$, alpha, beta , alpha_estimate_WGEE, beta_estimates_WGEE, splin_estimates_WGEE, std_alpha_estimate_WGEE, std_beta_estimates_WGEE)

WGEE_alpha<-RR_WGEE\$table_alpha_WGEE_splin

WGEE_beta<-RR_WGEE\$table_beta_WGEE_splin

WGEE_spline <-RR_WGEE\$table_splin_WGEE

\# $-\mathrm{GEE}$

RR_GEE<-Cal ( $z$, alpha, beta ,

alpha_estimate_GEE, beta_estimates_GEE, splin_estimates_GEE, std_alpha_estimate_GEE, std_beta_estimates_GEE)

GEE_alpha<-RR_GEE\$table_alpha_WGEE_splin 


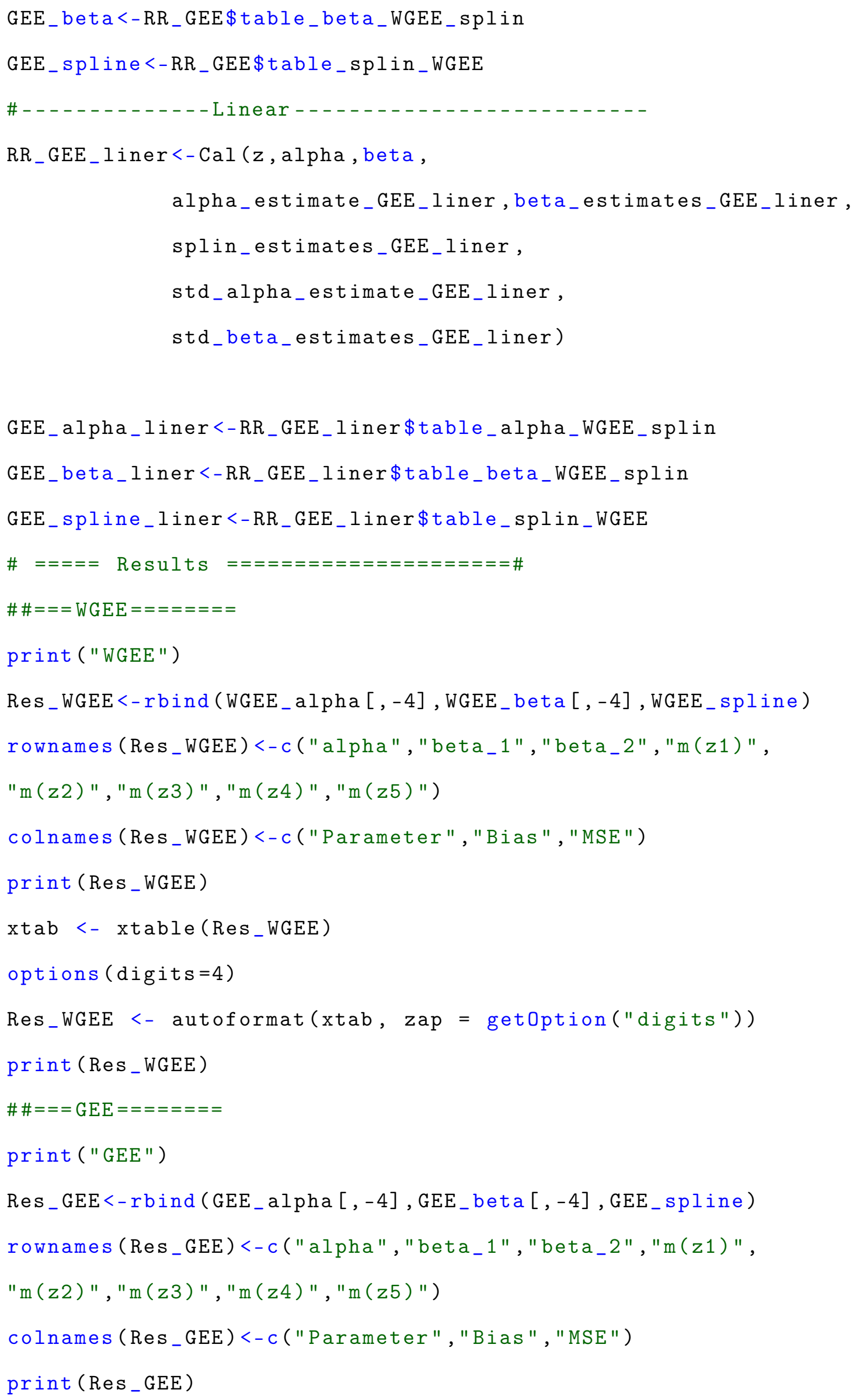




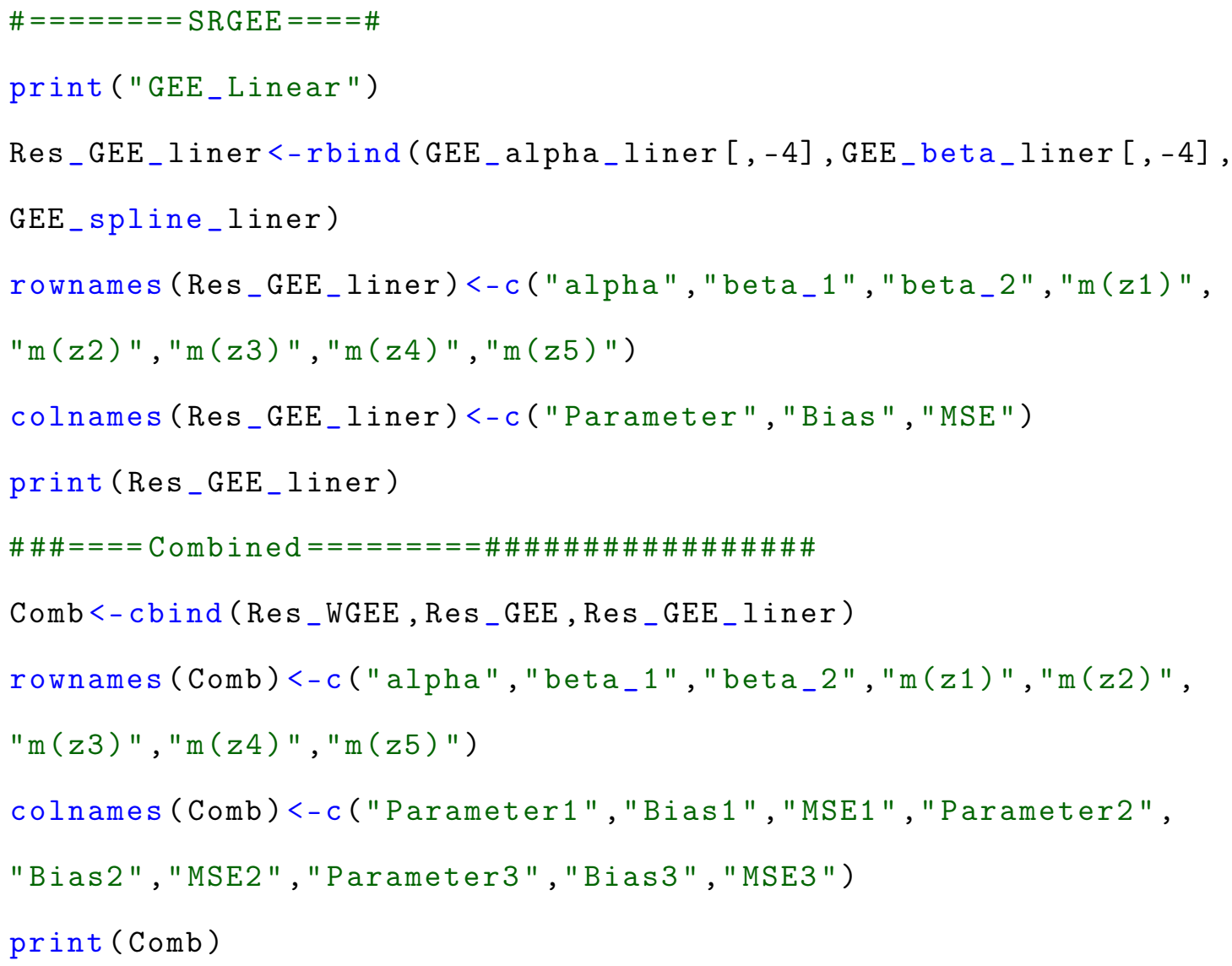




\section{Appendix B}

Additional Simulation Results 
Table B.1: Empirical biases and mean squared errors (MSEs) of GEE, RSGEE and WRSGEE estimators of regression and association and dispersion parameters.

\begin{tabular}{|c|c|c|c|c|c|c|c|}
\hline \multicolumn{8}{|c|}{ Partially Linear Model for $\boldsymbol{\tau}=(-3,0.8,0)$} \\
\hline \multirow[b]{2}{*}{ Parameter } & \multirow[b]{2}{*}{ True value } & \multicolumn{2}{|c|}{ GEE } & \multicolumn{2}{|c|}{ RSGEE } & \multicolumn{2}{|c|}{ WRSGEE } \\
\hline & & Bias & MSE & Bias & MSE & Bias & MSE \\
\hline \multicolumn{8}{|l|}{$k=300$} \\
\hline$\alpha$ & 0.16 & -0.0132 & 0.0065 & -0.0131 & 0.0066 & -0.0427 & 0.0209 \\
\hline$\phi$ & 1.2 & -0.1632 & 0.0310 & -0.1709 & 0.0335 & -0.1952 & 0.0553 \\
\hline$\beta_{1}$ & 0.5 & -0.0062 & 0.0024 & -0.0060 & 0.0024 & -0.0230 & 0.0109 \\
\hline$\beta_{2}$ & 0.2 & -0.0051 & 0.0014 & -0.0055 & 0.0014 & 0.0052 & 0.0041 \\
\hline$m\left(z_{1}\right)$ & 0.53 & 0.1326 & 0.0232 & 0.0678 & 0.0165 & 0.0294 & 0.0487 \\
\hline$m\left(z_{2}\right)$ & 0.55 & 0.1095 & 0.0176 & 0.0495 & 0.0132 & 0.0110 & 0.0441 \\
\hline$m\left(z_{3}\right)$ & 0.66 & 0.0178 & 0.0049 & 0.0397 & 0.0082 & 0.0023 & 0.0236 \\
\hline$m\left(z_{4}\right)$ & 0.68 & 0.0242 & 0.0049 & 0.0335 & 0.0069 & 0.0002 & 0.0257 \\
\hline$m\left(z_{5}\right)$ & 0.69 & 0.0511 & 0.0086 & 0.0340 & 0.0137 & -0.0119 & 0.0461 \\
\hline \multicolumn{8}{|l|}{$k=500$} \\
\hline$\alpha$ & 0.16 & -0.0073 & 0.0036 & -0.0067 & 0.0036 & -0.0336 & 0.0116 \\
\hline$\phi$ & 1.2 & -0.1605 & 0.0284 & -0.1657 & 0.0300 & -0.1561 & 0.0383 \\
\hline$\beta_{1}$ & 0.5 & -0.0063 & 0.0015 & -0.0062 & 0.0015 & -0.0218 & 0.0073 \\
\hline$\beta_{2}$ & 0.2 & -0.0025 & 0.0007 & -0.0027 & 0.0007 & 0.0051 & 0.0025 \\
\hline$m\left(z_{1}\right)$ & 0.53 & 0.1295 & 0.0203 & 0.0633 & 0.0119 & 0.0395 & 0.0345 \\
\hline$m\left(z_{2}\right)$ & 0.55 & 0.1065 & 0.0148 & 0.0450 & 0.0092 & 0.0212 & 0.0305 \\
\hline$m\left(z_{3}\right)$ & 0.66 & 0.0146 & 0.0030 & 0.0373 & 0.0052 & 0.0120 & 0.0166 \\
\hline$m\left(z_{4}\right)$ & 0.68 & 0.0210 & 0.0029 & 0.0305 & 0.0047 & 0.0018 & 0.0170 \\
\hline$m\left(z_{5}\right)$ & 0.69 & 0.0477 & 0.0056 & 0.0271 & 0.0076 & -0.0093 & 0.0268 \\
\hline \multicolumn{8}{|l|}{$k=1000$} \\
\hline$\alpha$ & 0.16 & -0.0097 & 0.0019 & -0.0093 & 0.0019 & -0.0306 & 0.0089 \\
\hline$\phi$ & 1.2 & -0.1623 & 0.0277 & -0.1653 & 0.0286 & -0.1235 & 0.0262 \\
\hline$\beta_{1}$ & 0.5 & -0.0059 & 0.0008 & -0.0058 & 0.0008 & -0.0202 & 0.0043 \\
\hline$\beta_{2}$ & 0.2 & -0.0044 & 0.0004 & -0.0044 & 0.0004 & 0.0048 & 0.0016 \\
\hline$m\left(z_{1}\right)$ & 0.53 & 0.1313 & 0.0189 & 0.0690 & 0.0082 & 0.0464 & 0.0209 \\
\hline$m\left(z_{2}\right)$ & 0.55 & 0.1083 & 0.0133 & 0.0506 & 0.0057 & 0.0277 & 0.0178 \\
\hline$m\left(z_{3}\right)$ & 0.66 & 0.0171 & 0.0016 & 0.0398 & 0.0035 & 0.0123 & 0.0112 \\
\hline$m\left(z_{4}\right)$ & 0.68 & 0.0242 & 0.0017 & 0.0319 & 0.0028 & 0.0058 & 0.0107 \\
\hline$m\left(z_{5}\right)$ & 0.69 & 0.0521 & 0.0044 & 0.0348 & 0.0049 & -0.0029 & 0.0180 \\
\hline \multicolumn{8}{|l|}{$\bar{k} k=5000$} \\
\hline$\alpha$ & 0.16 & -0.0102 & 0.0005 & -0.0101 & 0.0005 & -0.0217 & 0.0058 \\
\hline$\phi$ & 1.2 & -0.1614 & 0.0263 & -0.1628 & 0.0268 & -0.0622 & 0.0111 \\
\hline$\beta_{1}$ & 0.5 & -0.0061 & 0.0002 & -0.0061 & 0.0002 & -0.0082 & 0.0018 \\
\hline$\beta_{2}$ & 0.2 & -0.0045 & 0.0001 & -0.0046 & 0.0001 & 0.0007 & 0.0007 \\
\hline$m\left(z_{1}\right)$ & 0.53 & 0.1317 & 0.0177 & 0.0703 & 0.0057 & 0.0642 & 0.0132 \\
\hline$m\left(z_{2}\right)$ & 0.55 & 0.1087 & 0.0122 & 0.0518 & 0.0034 & 0.0454 & 0.0104 \\
\hline$m\left(z_{3}\right)$ & 0.66 & 0.0174 & 0.0006 & 0.0397 & 0.0019 & 0.0279 & 0.0061 \\
\hline$m\left(z_{4}\right)$ & 0.68 & 0.0245 & 0.0009 & 0.0335 & 0.0015 & 0.0239 & 0.0063 \\
\hline$m\left(z_{5}\right)$ & 0.69 & 0.0523 & 0.0031 & 0.0349 & 0.0019 & 0.0183 & 0.0096 \\
\hline
\end{tabular}




\section{Appendix C}

\section{Analysis of a subset of HRS data,}

\section{with the maximum number of doctor visits of 100 .}

Table C.1: ML estimates, standard errors and z-value of missing data model parameters for the HRS data analysis

\begin{tabular}{rrrr}
\hline \hline & Estimate & S.E. & z-value \\
\hline Intercept & -6.4464 & 0.33653 & -19.16 \\
Previous visits & 0.0170 & 0.00177 & 9.60 \\
Age & 0.5196 & 0.03981 & 13.05 \\
Cancer & 0.1449 & 0.06092 & 2.38 \\
Heart & 0.2541 & 0.05288 & 4.80 \\
Lung & 0.5846 & 0.06755 & 8.65 \\
Smoke & -0.0694 & 0.05023 & -1.38 \\
White & -0.1847 & 0.14614 & -1.26 \\
Black & 0.2122 & 0.16270 & 1.30 \\
Time & 0.3151 & 0.02931 & 10.75 \\
\hline
\end{tabular}




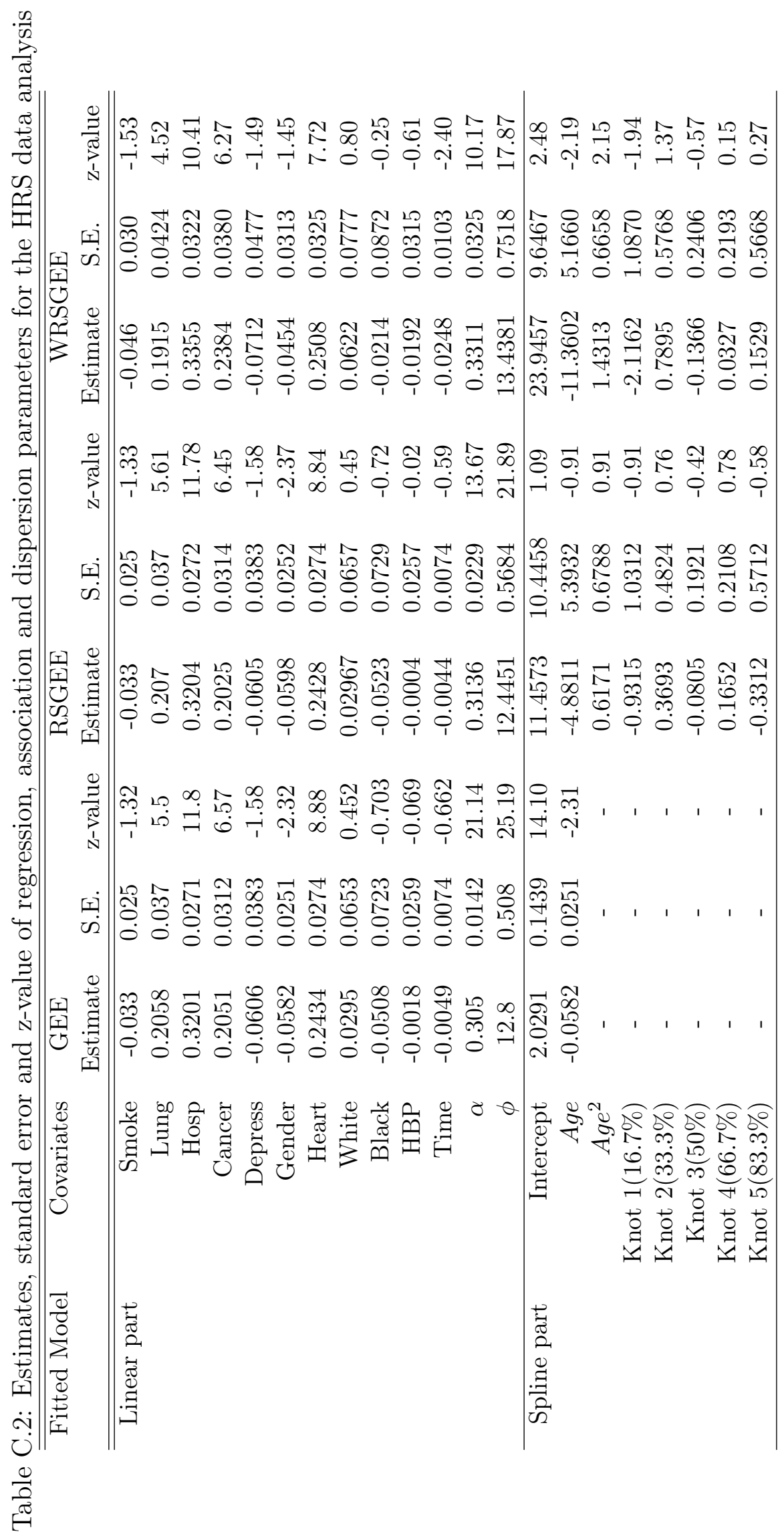


Table C.3: The nonlinear function $\mathrm{m}$ (Age) was estimated based on the spline function for different values of age

\begin{tabular}{lcccc}
\hline \hline & & GEE & RSGEE & WRSGEE \\
& Age & $\hat{m}$ (Age) & $\hat{m}$ (Age) & $\hat{m}$ (Age) \\
\hline \hline nonlinear function: & 4.1 & 2.06 & 1.82 & 1.43 \\
& 5.4 & 2.07 & 2.13 & 2.15 \\
& 6.2 & 2.08 & 2.10 & 2.10 \\
7.0 & 2.09 & 2.10 & 2.12 \\
8.0 & 2.10 & 2.13 & 2.11 \\
8.6 & 2.11 & 2.25 & 2.11 \\
\hline
\end{tabular}

